\title{
Orthogonal syntheses of 3.2.0 bicycles from enallenes promoted by visible light
}

\author{
Andrea Serafino, ${ }^{a}$ Davide Balestri, ${ }^{a}$ Luciano Marchiò, ${ }^{a}$ \\ Max Malacria, ${ }^{b}$ Etienne Derat, ${ }^{b}$ Giovanni Maestri ${ }^{a}$
}

a Università di Parma, Department of Chemistry, Life Sciences and Environmental Sustainability, Parco Area delle Scienze 17/A, 43124 Parma, Italy

b Sorbonne Université, Faculty of Science and Engineering, CNRS, Institut Parisien de Chimie Moléculaire (UMR CNRS 8232), 4 place Jussieu, 75252 Paris Cedex 05, France 
Table of contents

General remarks

Additional computational results 5

Selected optimization entries 7

Synthesis of substrates 9

Characterization of products 22

X-Ray crystallography data 33

Copies of NMR spectra 38

$\mathrm{XYZ}$ coordinates 94

Comprehensive table in $\mathrm{AU}$ 116

References

118 


\section{General remarks}

All chemicals those syntheses are not reported hereafter were purchased from commercial sources and used as received. Solvents were dried passing through alumina columns using an Inert ${ }^{\oplus}$ system and were stored under nitrogen. Present visible-light promoted reactions did not required the use of dry solvents but presence of molecular oxygen exerts a negative effect on their rate. Chromatographic purifications were performed under gradient using a Combiflash $^{\oplus}$ system and prepacked disposable silica cartridges or through isocratic flash chromatography using commercial $60 \AA$ silica gel. All reactions that required heating were performed with the use of high-vacuum grade silicon oil. Reactions promoted by visible light were carried out in standard 4mm NMR tubes, surrounded by a commercial strip of 300 RGB household leds (12V) that were put a distance of ca $10 \mathrm{~cm}$ and switched on irradiating white light (model SMD5050-300 ip65). The tubes were inside an oil bath fitted with a thermometer to monitor the temperature of the solution. To avoid reproducibility issues, cooling is ensured by two fans recovered by outdated PCs. During summer time, solutions are kept at RT through the addition, inside the silicon oil bath, of a rubber spire linked to a chiller, which keeps pumping a cooled ethanol solution to maintain the desired temperature.

${ }^{1} \mathrm{H}$ and ${ }^{13} \mathrm{C}$ NMR spectra were recorded at $300 \mathrm{~K}$ on a Bruker $300 \mathrm{MHz}$, a Bruker $400 \mathrm{MHz}$ or a Jeol $600 \mathrm{MHz}$ spectrometers using residual non-deuterated solvents as internal standards (7.26 ppm for ${ }^{1} \mathrm{H}$ NMR and 77.00 ppm for ${ }^{13} \mathrm{C}-\mathrm{NMR}$ for $\mathrm{CDCl}_{3}, 2.05 \mathrm{ppm}$ for ${ }^{1} \mathrm{H} \mathrm{NMR}$ and 29.84 ppm for ${ }^{13} \mathrm{C}$ NMR for acetone- $\left.\mathrm{d}_{6}\right) .{ }^{19} \mathrm{~F}-\mathrm{NMR}$ spectra were recorded in $\mathrm{CDCl}_{3}$ at $298 \mathrm{~K}$ on a Jeol 600 spectrometer fitted with a BBFO probehead at $564 \mathrm{MHz}$. The terms $\mathrm{m}, \mathrm{s}, \mathrm{d}, \mathrm{t}, \mathrm{q}$ and quint represent multiplet, singlet, doublet, triplet, quadruplet and quintuplet respectively, and the term br means a broad signal. Mass analyses were recorded on an Infusion Water Acquity Ultra Performance LC HO6UPS-823M instrument equipped with a SQ detector (Electrospray source); high-resolution mass analyses were recorded on a LTQ ORBITRAP XL Thermo Mass Spectrometer (Electrospray source).

CCDC 2009276, 2009277 and 2009278 contain the supplementary crystallographic data for compounds $\mathbf{2 a}, \mathbf{2}$ '-I and $\mathbf{4 a}$, respectively. Single crystal Data were collected with a Bruker D8 diffractometer equipped with Photonll area detector, using a CuKa or a MoKa microfocus 
radiation source. The data collection strategy covered the sphere of reciprocal space. Absorption corrections were applied using the program SADABS. The structure was solved with the SHELXT code. Fourier analysis and refinement were performed by the full-matrix least-squares methods based on F2 using SHELXL-2014 as implemented in Olex2. All the non$\mathrm{H}$ atoms were refined with anisotropic displacement parameters.

Calculations were performed with Gaussian 09 at the DFT level. ${ }^{[1]}$ The geometries of all species were optimized without any constraints at the generalized gradient approximation using the M06 hybrid functional of Zhao and Truhlar. ${ }^{[2]}$ On key intermediates and TSs, reoptimization with the M06-2X functional were carried out to assess the influence of different Hartree-Fock contributions to calculated energies and geometries. Optimizations were carried out using the LACVP $(d)$ basis set, which consists of the standard 6-31G(d) basis set for lighter atoms $(H, C$, $\mathrm{N}, \mathrm{O}$ ), and with the Def2-SVP(d) set defined by Weigand and Ahlrichs to check potential biases due to basis set effects. ${ }^{[3]}$ On Def2-SVP(d) optimized geometries, complete re-optimization was performed employing a more refined triple- $\zeta$ basis set, namely the def2-TZVP(d) set. ${ }^{[3]}$ These gas-phase structures were then freely optimized using DMF as an implicit solvent as implemented in Gaussian 09 through the CPCM approach. ${ }^{[4]}$ The starting approximate geometries for transition states (TS) were obtained through scans of the relative reaction coordinate. Transition states were identified by the presence of a single imaginary frequency in the Hessian matrix, corresponding to the vibration along the reaction coordinate linking the reactant and the product. Conversely, stable intermediates were identified by the absence of imaginary frequencies in their Hessian matrix. Additional modeled pathways that proved less feasible are briefly presented hereafter. XYZ coordinates and a comprehensive table in atomic units including imaginary IR frequencies for transition states are in the dedicated section of present documents. 


\section{Additional computational results}

- Less favorable reaction pathways

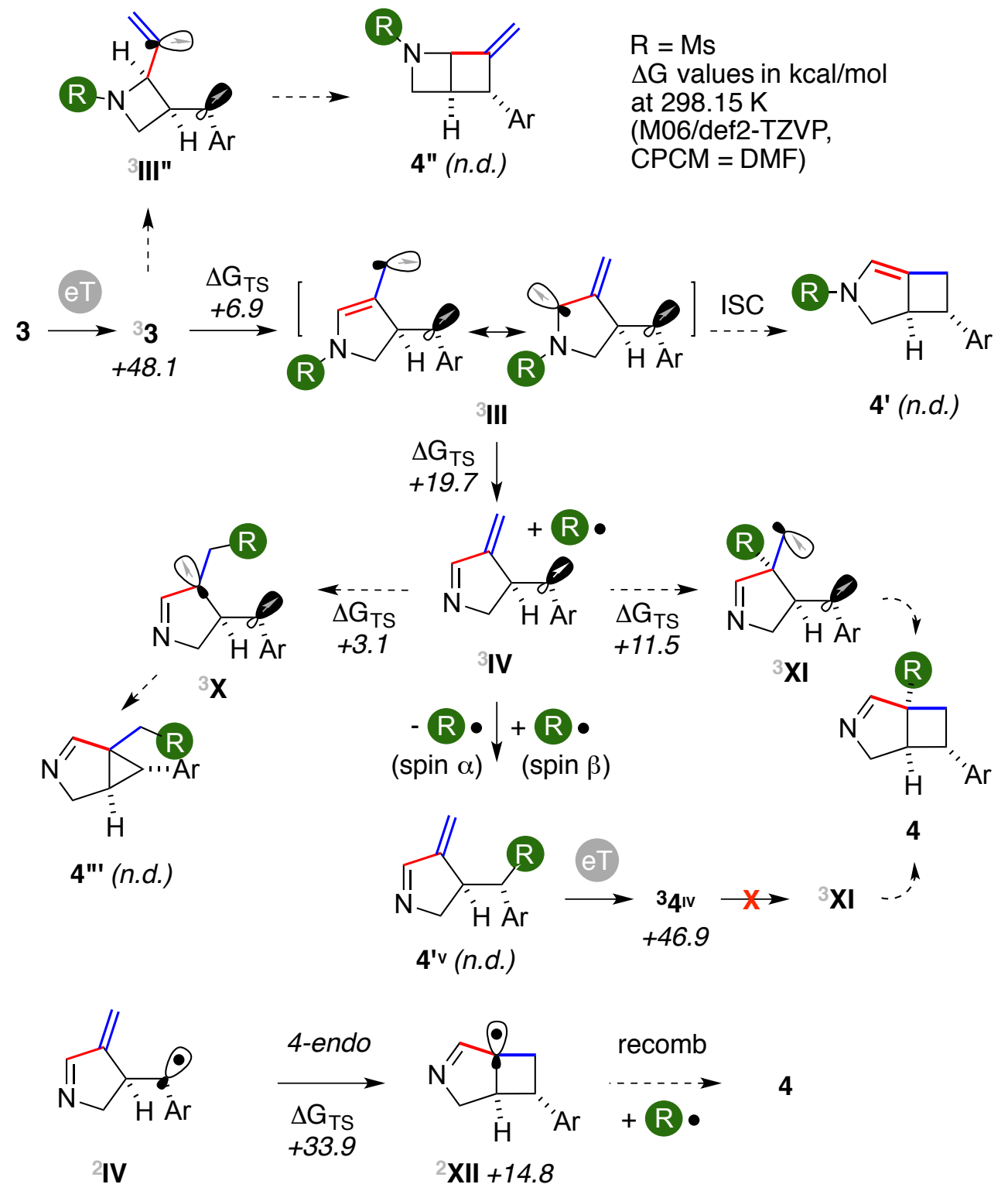

Radical addition on the C-C double bond of ${ }^{3} \mathrm{IV}$ can occur from its least hindered face. The two competing pathways have both low barriers ( +3.1 and $+11.5 \mathrm{kcal} / \mathrm{mol}$, respectively), but that on the terminal carbon atom results way more favorable (by $-8.2 \mathrm{kcal} / \mathrm{mol}$ in $\Delta \Delta \mathrm{G}$ at the M06/Def2-tzvp level). This is due to both a reduced hindrance and a higher stabilization of the resulting $\alpha$-imino tertiary radical in ${ }^{3} X$. Although this feature is consistent with ample literature precedents, it is starkly at odds with the recovery of product 4 and the absence of the 3.1.0 bicycle 4'". None of them can either undergo further activation from PC1 via eT according to modeling. Recombination of the two fragments of triplet ${ }^{3} \mathrm{II}$ is forbidden if both have the same 
spin. It is however conceivable, on the basis of the experimental findings revealing a multimolecular mechanism for the formal fragmentation/addition of the sulfonyl group, that formation of $4^{\mathrm{IV}}$ could occur via recombination of two radicals with different spin. A second excitation of this putative intermediate could lead to the corresponding triplet ${ }^{3} 4^{I V}$. This energy gap is similar to those calculated for the reagent itself and seems therefore accessible under present condition $(+46.9 \mathrm{kcal} / \mathrm{mol}$ in $\Delta \mathrm{G})$. Try as we might, we were however unable to find any accessible transition state for the subsequent 1,3-sulfonyl migration from the benzylic position to carbon atom that occupies the headbridge position in the final product 4 . A similar reaction would have led to triplet ${ }^{3} \mathrm{XI}$, which could have been delivered the final product upon ISC. Finally, an intramolecular 4-endo-trig cyclyzation on radical ${ }^{2} \mathrm{IV}$ has been modeled. The calculated barrier is significantly high in energy $(\Delta G=+33.9 \mathrm{kcal} / \mathrm{mol})$ and the resulting intermediate ${ }^{2} \mathrm{XII}$ is less stable than the entry channel (by $+14.8 \mathrm{kcal} / \mathrm{mol}$ in $\Delta G$ ). Although radical recombination of this species with a sulfonyl should be energetically favorable, modelled values for this 4-endo-trig are significantly higher than those presented in the main article. This reduces the odds that a similar pathway takes place in present reactions.

- Basis set and Hartree-Fock contribution effects on N-S fragmentation

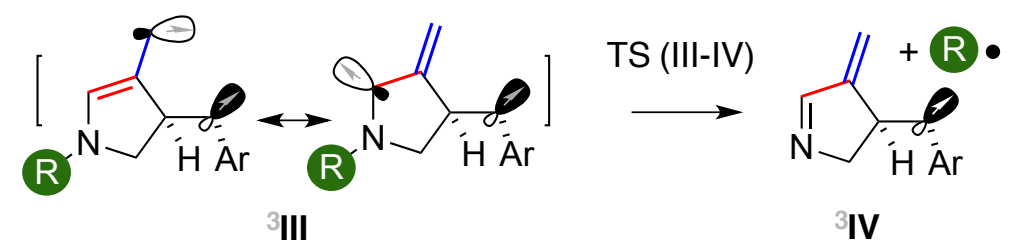

The fragmentation of ${ }^{3} \mathrm{III}$ and the corresponding TS (III-IV) have been modeled using three different basis sets (def2-SVP, def-TZVP and $\operatorname{lacvp}(\mathrm{d})$ ) and two different functionals with different Hartree-Fock contributions (M06 and M06-2X). In contrast to the other steps of present reactions, a higher than usual difference (up to $8 \mathrm{kcal} / \mathrm{mol}$ in $\Delta \mathrm{G}$ ) emerged among these TSs. This seems to correlate with the calculated N-S bond length in intermediate ${ }^{3} \mathrm{III}$. Although variation ranges 0.01-0.03 angstroms, a slightly longer bond in the intermediate leads to lower calculated barrier for the fragmentation. The following table sum up calculated data for these different systems. 


\begin{tabular}{|c|c|c|c|c|c|c|c|}
\hline \multicolumn{8}{|l|}{ CPCM (DMF) } \\
\hline M06/def2-SVP & H (Hartrees) & $\begin{array}{c}\mathrm{S} \\
\text { (cal/K* } \mathrm{Kol})\end{array}$ & $\begin{array}{c}\text { Imaginary freq } \\
(1 / \mathrm{cm})\end{array}$ & $\Delta \mathrm{H}$ & $\Delta \mathrm{S}$ & $\Delta \mathrm{G}$ & $\begin{array}{l}\text { Bond lenght } \\
\text { (Ang) }\end{array}$ \\
\hline${ }^{3} I I I$ & -1106.243898 & 134.23 & & & & & $\begin{array}{c}N S= \\
1.67917\end{array}$ \\
\hline TS (III-IV) & -1106.220223 & 135.973 & -313.6115 & 14.86 & 1.743 & 14.34 & \\
\hline \multicolumn{8}{|c|}{ M062X/def2-SVP } \\
\hline${ }^{3} \mathrm{III}$ & -1106.416324 & 131.045 & & & & & $\begin{array}{c}N S= \\
1.66719\end{array}$ \\
\hline TS (III-IV) & -1106.389236 & 136.275 & -383.6849 & 17.00 & 5.230 & 15.44 & \\
\hline \multicolumn{8}{|l|}{ M06/def2-TZVP } \\
\hline${ }^{3} \mathrm{III}$ & -1107.217777 & 133.285 & & & & & $\begin{array}{c}N S= \\
1.64974\end{array}$ \\
\hline TS (III-IV) & -1107.185694 & 134.697 & -280.4895 & 20.13 & 1.412 & 19.71 & \\
\hline \multicolumn{8}{|l|}{$\begin{array}{l}\text { M062X/def2- } \\
\text { TZVP }\end{array}$} \\
\hline${ }^{3} \mathrm{III}$ & -1107.409185 & 130.902 & & & & & $\begin{array}{c}N S= \\
1.64303\end{array}$ \\
\hline TS (III-IV) & -1107.372658 & 134.440 & -291.8977 & 22.92 & 2.538 & 22.16 & \\
\hline \multicolumn{8}{|l|}{ M06/lacvp(d) } \\
\hline${ }^{3} \mathrm{III}$ & -1106.875924 & 134.063 & & & & & $\begin{array}{c}N S= \\
1.67725\end{array}$ \\
\hline TS (III-IV) & -1106.856009 & 135.658 & -286.159 & 12.50 & 1.595 & 12.02 & \\
\hline \multicolumn{8}{|l|}{ M062X/lacvp(d) } \\
\hline${ }^{3} \mathrm{III}$ & -1107.050056 & 130.835 & & & & & $\begin{array}{c}N S= \\
1.66768\end{array}$ \\
\hline TS (III-IV) & -1107.028059 & 134.555 & -365.0586 & 13.80 & 3.720 & 12.69 & \\
\hline
\end{tabular}




\section{Selected optimization entries}<smiles>C=C=CN([O])C/C=C\[Al]</smiles>

3a
Photocat (X mol\%), visible light

$\mathrm{N}_{2}$, solvent, $25^{\circ} \mathrm{C}$ 24 hours

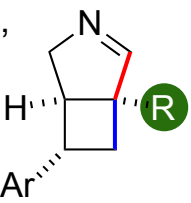

$4 a$

\begin{tabular}{|c|c|c|c|}
\hline entry & Photocat (mol\%) & Dry solvent (M of $3 a)$ & Yield of $\mathbf{4}^{b}$ \\
\hline 1 & Complex A (2.5\%) & $\operatorname{DMF}(0.2)$ & $41 \%$ \\
\hline $2^{a}$ & Complex A (2.5\%) & DMF (0.2) & $40 \%$ \\
\hline 3 & Complex A (1\%) & $\operatorname{DMF}(0.2)$ & $43 \%$ \\
\hline 4 & Complex A (0.5\%) & DMF (0.2) & $37 \%$ \\
\hline 5 & $\operatorname{Ir}(p p y)_{3}(1 \%)$ & DMF (0.2) & $36 \%(28 \%)$ \\
\hline 6 & $\mathrm{Ru}(\mathrm{bpy})_{3} \mathrm{Cl}_{2}(2.5 \%)$ & DMF (0.2) & -- \\
\hline 7 & Complex A (1\%) & Toluene/DCM 3:1 (0.20) & $52 \%$ \\
\hline 8 & Complex A (1\%) & Toluene/DCM 3:1 (0.05) & $62 \%$ \\
\hline 9 & Complex A (1\%) & $\operatorname{DMF}(0.05)$ & $68 \%(59 \%)$ \\
\hline 10 & $\operatorname{Ir}(p-F-p p y)_{3}(1 \%)$ & Toluene (0.05) & $54 \%$ \\
\hline
\end{tabular}

Reaction conditions: $0.1 \mathrm{mmol}$ of 3a, photocatalyst $(\mathrm{X} \mathrm{mol} \%)$, solvent $(0.05-0.2 \mathrm{M})$, in a $5 \mathrm{~mm}$ NMR tube at $25^{\circ} \mathrm{C}$ for 24 hours without stirring; ${ }^{a}$ at $45^{\circ} \mathrm{C} ;{ }^{1}{ }^{1} \mathrm{H}$ NMR yield using trimethoxybenzene as internal standard, isolated yields in parentheses.

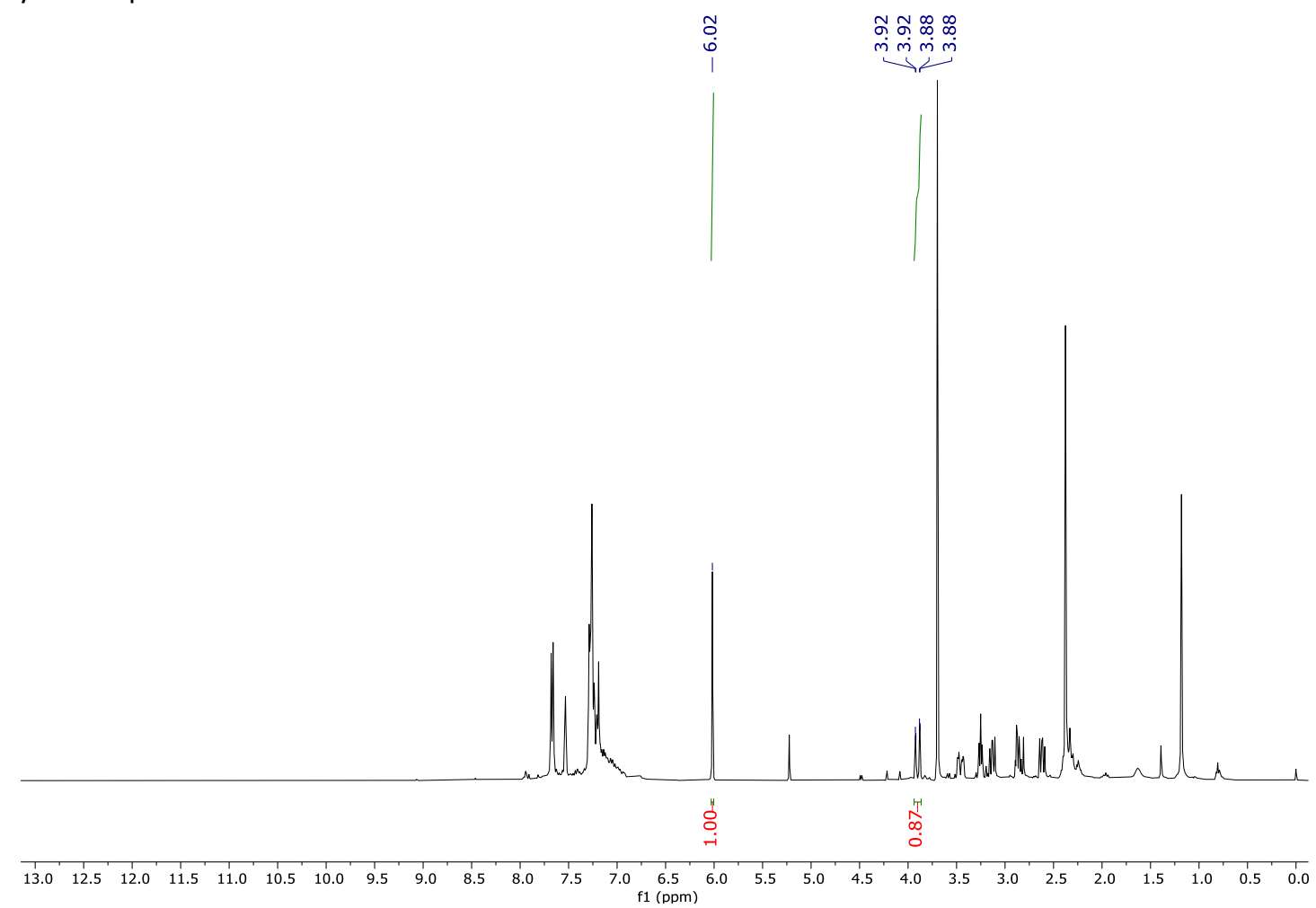

Copy of the ${ }^{1} \mathrm{H}$ NMR spectrum of the crude reaction mixture from entry 9 . Trimethoxybenzene $(2.36$ $\mathrm{mg}, 0.026 \mathrm{mmol}$, left peak) vs product (right peak). Beside $4 \mathrm{a}$, we were unable to identify any other well-defined byproduct of the reaction, although partial broadening of the aromatic region suggests that partial oligo-/polymerization of the enallene occurred. 


\title{
Synthesis of substrates
}

\author{
Synthesis of 1,6-enynes
}

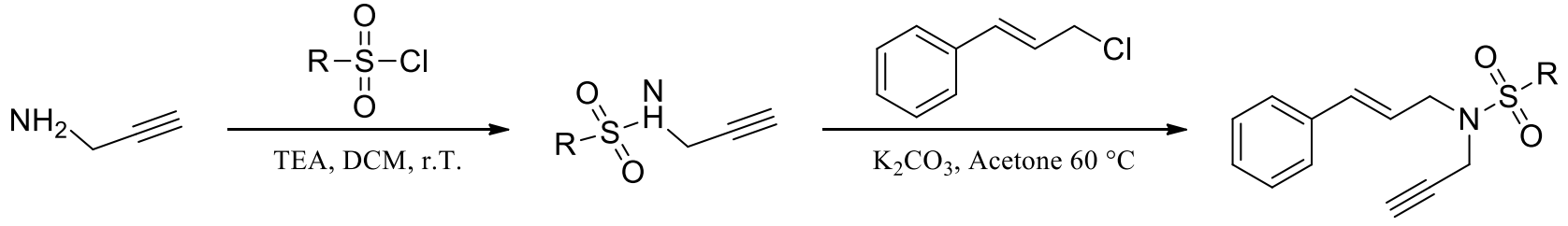

DCM (0.4 M), TEA (1.1 eq) and propargyl amine (1 equiv.) were added to a $100 \mathrm{~mL}$ round bottom flask equipped with magnetic stirring bar. The resulting mixture was stirred at $0{ }^{\circ} \mathrm{C}$ and sulfonyl halide $(1.05 \mathrm{eq})$ was added slowly. The reaction mixture was stirred at room temperature for 12 hours, quenched with sat $\mathrm{NH}_{4} \mathrm{Cl}$, diluted with $\mathrm{DCM}$ and washed with water. The organic layer was dried over $\mathrm{Na}_{2} \mathrm{SO}_{4}$, filtered and concentred under reduced pressure. Whitout isolation, propargyl tosylamide was transferred in a Schlenk tube equipped with a magnetic stirring bar and $\mathrm{K}_{2} \mathrm{CO}_{3}(1.5$ equiv.). Acetone $(0.33 \mathrm{M})$ and cinnamyl chloride (1.3 equiv.) were then added. The reaction mixture was stirred at $60^{\circ} \mathrm{C}$ overnight. The mixture was cooled to room temperature, diluted with water and extracted with EtOAc $(3 \times 20 \mathrm{~mL})$. The combined organic layers were dried over $\mathrm{Na}_{2} \mathrm{SO}_{4}$, filtered and concentred under reduced pressure. The resulting crude was purified by chromatography on silica gel (n-hexane/EtOAc 9:1).
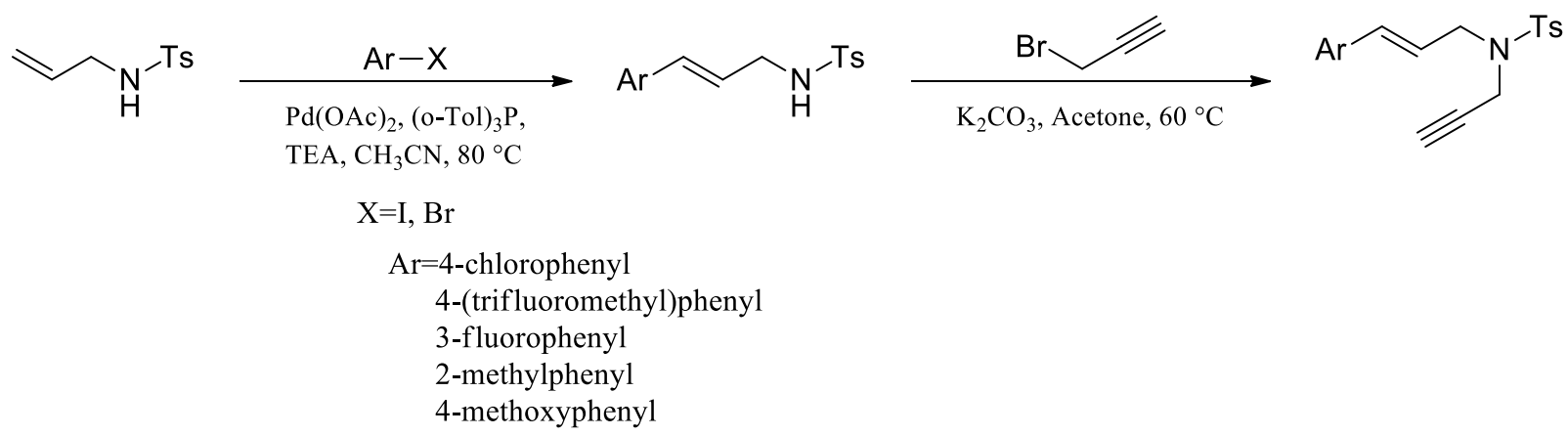

$\mathrm{N}$-allyl-4-methylbenzenesulfonamide (1 equiv.), (o-Tol) ${ }_{3} \mathrm{P}$ (0.1 equiv.) and $\mathrm{Pd}(\mathrm{OAc})_{2}(0.05$ equiv.) were sequentially added to a Schlenk tube equipped with magnetic stirring bar. $\mathrm{CH}_{3} \mathrm{CN}$ (0.41 M), TEA (2 equiv.) and the desired aryl halide (1 equiv.) were added under $\mathrm{N}_{2}$ atmosphere and the mixture was stirred at $80^{\circ} \mathrm{C}$ for 3 hours. A second batch of the desired aryl halide ( 0.42 equiv.), $\mathrm{Pd}(\mathrm{OAc})_{2}(0.026 \text { equiv.) and (o-Tol })_{3} \mathrm{P}(0.05$ equiv.) were then added. The mixture was stirred at $80{ }^{\circ} \mathrm{C}$ for further 6 hours, allowed to cool to room temperature, diluted with water and extracted with EtOAc $(3 \times 30 \mathrm{~mL})$. The combined organic layers were dried over $\mathrm{Na}_{2} \mathrm{SO}_{4}$, filtered and concentred under reduced pressure. The resulting crude was purified by chromatography on silica gel (n-hexane/EtOAc 9:1). The substituted cinnamyl tosylamide (1 equiv.) was dissolved in acetone ( $0.2 \mathrm{M}) . \mathrm{K}_{2} \mathrm{CO}_{3}$ (3 equiv.) and propargyl bromide ( $85 \%$ in toluene, 1.5 equiv.) were then added. The mixture was subsequently placed in a preheated oil bath at $60^{\circ} \mathrm{C}$ and stirred overnight. After consumption of the starting material, the reaction mixture was cooled down to room temperature and water was added. The mixture was extracted with EtOAc $(3 \times 30 \mathrm{~mL})$. The combined organic fractions were dried over $\mathrm{Na}_{2} \mathrm{SO}_{4}$ 
and concentrated under reduced pressure. The resulting crude was purified by chromatography on silica gel (n-hexane/EtOAc 8:2).
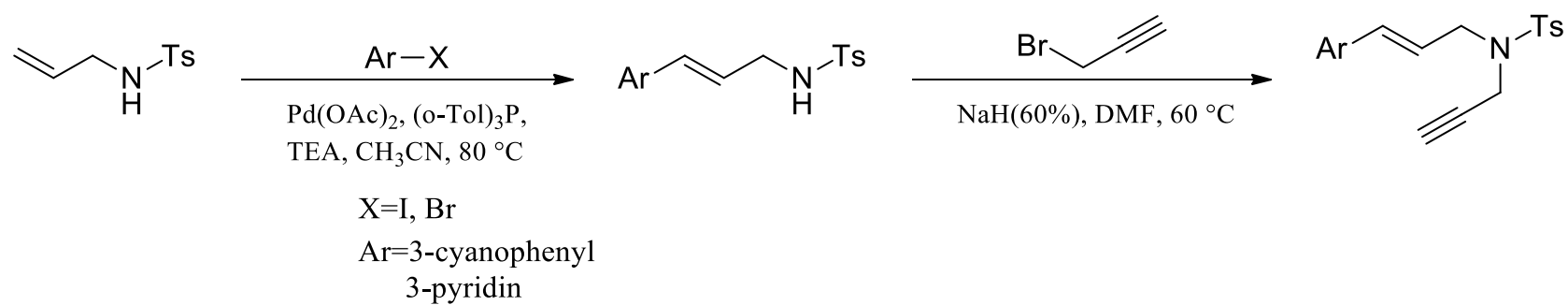

The Heck-type coupling was performed according to the above-mentioned procedure.

Then, the resulting tosylamide (1 equiv.) was dissolved in DMF $(0.2 \mathrm{M})$. NaH $(60 \%$ in mineral oil, 1.3 equiv.) was added slowly at $0^{\circ} \mathrm{C}$ under vigorous stirring. The resulting mixture was stirred for 1 hour at room temperature prior to the addition of a propargyl bromide solution ( $85 \%$ in toluene, 1.5 equiv.). The resulting mixture was then heated at $60^{\circ} \mathrm{C}$ for 2 hours. After completion, the reaction mixture was cooled down to room temperature and water was added. The mixture was extracted with EtOAc $(3 \times 30 \mathrm{~mL})$ and the organic layers washed with a sat. $\mathrm{LiCl}$ solution. The combined organic fractions were dried over $\mathrm{Na}_{2} \mathrm{SO}_{4}$ and concentrated under reduced pressure. The resulting crude was purified by chromatography on silica gel ( $n$ hexane/EtOAc 8:2).<smiles>C#CCN([12F])CC/C=C/c1ccccc1</smiles>

$\mathrm{Fe}(\mathrm{OTf})_{2}$ (0.1 equiv.) and toluene $(0.17 \mathrm{M})$ were added to a Schlenk tube equipped with magnetic stirring bar. The resulting mixture was stirred at room temperature for 10 minutes prior to the addition of a solution of cyclopropyl(phenyl)methanol (1 equiv.) and 4-methyl-N(prop-2-yn-1-yl)benzenesulfonamide (4 equiv.) in toluene $(0.17 \mathrm{M})$. The mixture was stirred at $100{ }^{\circ} \mathrm{C}$ and conversion was monitoring by TLC. The reaction mixture was then cooled and the solvent removed under reduced pressure. The resulting crude was purified by chromatography on silica gel (n-hexane/EtOAc 9:1).

Synthesis of 1,7-enallenes

General procedure 1a (GP-1a)<smiles>C#CCN([12CH3])/C=C/c1ccccc1/C=C/CN([12CH3])C/C=C/c1ccccc1</smiles>

Cul ( 0.1 equiv.), paraformaldehyde (1.6 equiv.), the desired 1,6-enyne (1 equiv.) and dioxane (1.5 M) were sequentially added to a $50 \mathrm{~mL}$ two-necked round-bottom flask equipped with a magnetic stirring. The resulting mixture was stirred at room temperature for 10 minutes prior to the addition of $i \operatorname{Pr}_{2} \mathrm{NH}$ (1.4 equiv.). The mixture was then heated to $110^{\circ} \mathrm{C}$ for 12 hours 
under air and then cooled down to room temperature. A sat. $\mathrm{NH}_{4} \mathrm{Cl}$ solution was added. The mixture was diluted with water and extracted with EtOAc $(3 \times 20 \mathrm{~mL})$. The combined organic layers were dried over $\mathrm{Na}_{2} \mathrm{SO}_{4}$, filtered and concentred under reduced pressure. The resulting crude was purified by chromatography on silica gel ( $n$-hexane/EtOAc 95:5).

\section{General procedure 1b (GP-1b)}

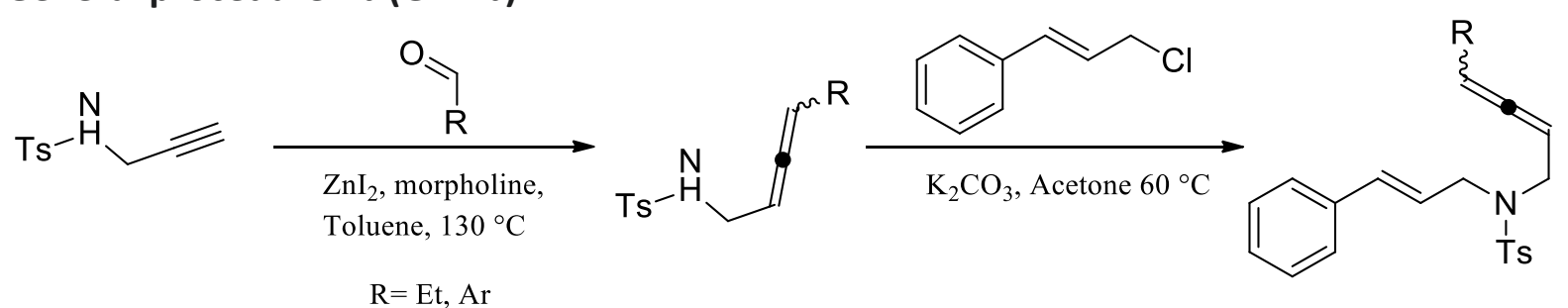

$\mathrm{ZnI}_{2}$ (0.8 equiv.), the desired aldehyde (1.8 equiv.), 4-methyl-N-(prop-2-yn-1yl)benzenesulfonamide (1 equiv.) and toluene $(0.33 \mathrm{M})$ were sequentially added to a $50 \mathrm{~mL}$ two-necked round-bottom flask equipped with a magnetic stirring bar. The resulting mixture was stirred at room temperature for 10 minutes prior to the addition of morpholine (1.4 equiv.). The mixture was then heated to $110^{\circ} \mathrm{C}$ for 12 hours under air and then cooled down to room temperature. A saturated $\mathrm{NH}_{4} \mathrm{Cl}$ solution was added. The mixture was diluted with water and extracted with EtOAc $(3 \times 20 \mathrm{~mL})$. The combined organic layer was dried over $\mathrm{Na}_{2} \mathrm{SO}_{4}$, filtered and concentred under reduced pressure. The resulting crude was purified by chromatography on silica gel (n-hexane/EtOAc 8:2). The intermediate was dissolved in acetone $(0.33 \mathrm{M}) . \mathrm{K}_{2} \mathrm{CO}_{3}$ (1.5 equiv.) and cinnamylchloride (1.3 equiv.) were sequentially added. The mixture was stirred at $60{ }^{\circ} \mathrm{C}$ monitoring the conversion by TLC. Upon full conversion, the mixture was diluted with EtOAc $(20 \mathrm{~mL})$ and washed with water. The combined organic layers were dried over $\mathrm{Na}_{2} \mathrm{SO}_{4}$, filtered and concentred under reduced pressure. The resulting crude was purified by chromatography on silica gel (n-hexane/EtOAc $95: 5) \cdot{ }^{[5]}$

\section{Synthesis of 1,6-enallenes}

\section{General procedure 2 (GP-2)}

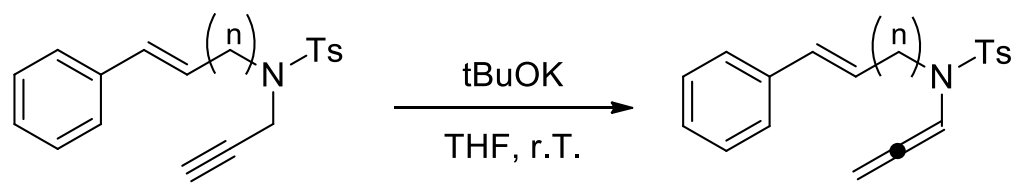

$\mathrm{n}=1,2$

The desired alkyne ( 1 equiv.) and THF $(0.17 \mathrm{M})$ were sequentially added to a Schlenk tube equipped with a magnetic stirring bar. the resulting mixture was stirred at room temperature for 10 minutes prior to the addition of tBuOK ( 0.3 equiv.). After complete conversion as monitored by TLC, a saturated $\mathrm{NH}_{4} \mathrm{Cl}$ solution $(15 \mathrm{ml}$ ) was added. The mixture was extracted with EtOAc $(3 \times 15 \mathrm{~mL})$, the organic layers separated and dried over $\mathrm{Na}_{2} \mathrm{SO}_{4}$. The solution was concentrated under reduced pressure and the crude purified by chromatography on silica gel (n-hexane/EtOAc 95:5). 
<smiles>C=CCN([125I])C/C=C/c1ccccc1</smiles>

The general procedure (GP-1a) was followed using the correspondent enyne (600mg, 1.85 mmol). Purification by chromatography on silica gel yielded 1a (182.7 mg, 29\%) as a white solid. ${ }^{1} \mathrm{H}$ NMR $\left(400 \mathrm{MHz}, \mathrm{CDCl}_{3}\right) \delta=7.71(\mathrm{~d}, J=8.3 \mathrm{~Hz}, 2 \mathrm{H}), 7.50-6.73(\mathrm{~m}, 7 \mathrm{H}), 6.43(\mathrm{~d}, J=$ $15.8 \mathrm{~Hz}, 1 \mathrm{H}), 5.96(\mathrm{dt}, J=15.8,6.8 \mathrm{~Hz}, 1 \mathrm{H}), 4.91(\mathrm{p}, J=6.9 \mathrm{~Hz}, 1 \mathrm{H}), 4.67(\mathrm{dt}, J=6.6,2.5 \mathrm{~Hz}, 2 \mathrm{H})$, $3.98(\mathrm{~d}, J=6.8, \mathrm{~Hz}, 2 \mathrm{H}), 3.87(\mathrm{dt}, J=7.2,2.5 \mathrm{~Hz}, 2 \mathrm{H}), 2.40(\mathrm{~s}, 3 \mathrm{H}) .{ }^{13} \mathrm{C}$ NMR $\left(101 \mathrm{MHz}, \mathrm{CDCl}_{3}\right) \delta$ $=209.6(\mathrm{Cq}), 143.4(\mathrm{Cq}), 137.5(\mathrm{Cq}), 136.3(\mathrm{Cq}), 134.2(\mathrm{CH}), 129.8(\mathrm{CH}), 128.6(\mathrm{CH}), 127.9(\mathrm{CH})$, $127.3(\mathrm{CH}), 126.5(\mathrm{CH}), 123.7(\mathrm{CH}), 85.8(\mathrm{CH}), 76.3\left(\mathrm{CH}_{2}\right), 48.9\left(\mathrm{CH}_{2}\right), 45.8\left(\mathrm{CH}_{2}\right), 21.6\left(\mathrm{CH}_{3}\right)$. (ESI)-HRMS calcd for $\mathrm{C}_{20} \mathrm{H}_{22} \mathrm{NO}_{2} \mathrm{~S}[\mathrm{M}+\mathrm{H}]^{+} 340.1366$, found 340.1374 .

(E)-N-(buta-2,3-dien-1-yl)-N-(3-(4-chlorophenyl)allyl)-4-methylbenzenesulfonamide (1b)<smiles>C=CCN([125I])C/C=C/c1ccc(Cl)cc1</smiles>

The general procedure (GP-1a) was followed using the correspondent enyne (312 mg, 0.87 moml). Purification by chromatography on silica gel yielded $\mathbf{1 b}(197 \mathrm{mg}, 61 \%)$ as a white solid. ${ }^{1} \mathrm{H}$ NMR (300 MHz, acetone) $\delta=7.83-7.73(\mathrm{~m}, 2 \mathrm{H}), 7.48-7.27(\mathrm{~m}, 6 \mathrm{H}), 6.56(\mathrm{dt}, \mathrm{J}=15.8,1.5$ $\mathrm{Hz}, 1 \mathrm{H}), 6.10(\mathrm{dt}, J=15.9,6.7 \mathrm{~Hz}, 1 \mathrm{H}), 4.99(\mathrm{p}, J=6.9 \mathrm{~Hz}, 1 \mathrm{H}), 4.75(\mathrm{dt}, J=6.6,2.5 \mathrm{~Hz}, 2 \mathrm{H}), 4.02$ $(\mathrm{d}, J=6.6 \mathrm{~Hz}, 2 \mathrm{H}), 3.90(\mathrm{dt}, J=7.1,2.6 \mathrm{~Hz}, 2 \mathrm{H}), 2.42(\mathrm{~s}, 3 \mathrm{H}) .{ }^{13} \mathrm{C} \mathrm{NMR}(75 \mathrm{MHz}$, acetone) $\delta=$ $209.5(\mathrm{Cq}), 143.4(\mathrm{Cq}), 137.8(\mathrm{Cq}), 135.5(\mathrm{Cq}), 132.9(\mathrm{Cq}), 132.4(\mathrm{CH}), 129.8(\mathrm{CH}), 128.6(\mathrm{CH})$, $128.0(\mathrm{CH}), 127.3(\mathrm{CH}), 125.1(\mathrm{CH}), 85.6(\mathrm{CH}), 75.6\left(\mathrm{CH}_{2}\right), 48.7\left(\mathrm{CH}_{2}\right), 45.9\left(\mathrm{CH}_{2}\right), 20.6\left(\mathrm{CH}_{3}\right)$. (ESI)-HRMS calcd for $\mathrm{C}_{20} \mathrm{H}_{20} \mathrm{CINNaO}{ }_{2} \mathrm{~S}[\mathrm{M}+\mathrm{Na}]^{+} 396.0795$, found 396.0791 .

(E)-N-(buta-2,3-dien-1-yl)-4-methyl-N-(3-(4-

(trifluoromethyl)phenyl)allyl)benzenesulfonamide (1c)<smiles>C=CCN([AlH])C/C=C/c1ccc(C(F)(F)F)cc1</smiles>

The general procedure (GP-1a) was followed using the correspondent enyne $(310 \mathrm{mg}, 0.82$ $\mathrm{mmol})$. Purification by chromatography on silica gel yielded $1 \mathrm{c}(212 \mathrm{mg}, 66 \%)$ as a white solid. ${ }^{1} \mathrm{H}$ NMR $\left(400 \mathrm{MHz}^{\mathrm{CDCl}}{ }_{3}\right) \delta=7.76-7.70(\mathrm{~m}, 2 \mathrm{H}), 7.55(\mathrm{~d}, J=8.1 \mathrm{~Hz}, 2 \mathrm{H}), 7.37(\mathrm{~d}, J=8.1 \mathrm{~Hz}$, $2 \mathrm{H}), 7.30(\mathrm{~d}, J=8.0 \mathrm{~Hz}, 2 \mathrm{H}), 6.50(\mathrm{~d}, J=15.9 \mathrm{~Hz}, 1 \mathrm{H}), 6.12(\mathrm{dt}, J=15.9,6.6 \mathrm{~Hz}, 1 \mathrm{H}), 4.96(\mathrm{p}, J=$ $6.8 \mathrm{~Hz}, 1 \mathrm{H}), 4.69(\mathrm{dt}, J=6.5,2.5 \mathrm{~Hz}, 2 \mathrm{H}), 4.02(\mathrm{~d}, J=6.6 \mathrm{~Hz}, 2 \mathrm{H}), 3.89(\mathrm{dt}, J=7.1,2.5 \mathrm{~Hz}, 2 \mathrm{H})$, $2.42(\mathrm{~s}, 3 \mathrm{H}) .{ }^{13} \mathrm{C}$ NMR (101 MHz, CDCl 3$) \delta=209.7(\mathrm{Cq}), 143.5(\mathrm{Cq}), 139.8(\mathrm{Cq}), 137.3(\mathrm{Cq}), 132.4$ $(\mathrm{CH}), 129.8(\mathrm{CH}), 129.6(\mathrm{Cq}, J=32.1 \mathrm{~Hz}), 127.2(\mathrm{CH}), 126.8(\mathrm{CH}), 126.6(\mathrm{CH}), 125.5(\mathrm{Cq}, J=4.1$ $\mathrm{Hz}), 124,1(\mathrm{Cq}, J=271.5 \mathrm{~Hz}), 85.7(\mathrm{CH}), 76.3\left(\mathrm{CH}_{2}\right), 48.7\left(\mathrm{CH}_{2}\right), 46.2\left(\mathrm{CH}_{2}\right), 21.5\left(\mathrm{CH}_{3}\right) .{ }^{19} \mathrm{~F} \mathrm{NMR}$ 
(564 MHz, $\mathrm{CDCl}_{3}$ ) $\delta=-62.44$. (ESI)-HRMS calcd for $\mathrm{C}_{21} \mathrm{H}_{20} \mathrm{~F}_{3} \mathrm{NNaO}_{2} \mathrm{~S}[\mathrm{M}+\mathrm{Na}]^{+} 430.1059$, found 430.1048 .

(E)-N-(buta-2,3-dien-1-yl)-N-(3-(3-fluorophenyl)allyl)-4-methylbenzenesulfonamide (1d)<smiles>C=CCN([13CH3])C/C=C/c1cccc(F)c1</smiles>

The general procedure (GP-1a) was followed using the correspondent enyne ( $240 \mathrm{mg}, 0.7$ mmol). Purification by chromatography on silica gel yielded $1 \mathbf{d}(102 \mathrm{mg}, 41 \%)$ as a white solid. ${ }^{1} \mathrm{H}$ NMR $\left(400 \mathrm{MHz}, \mathrm{CDCl}_{3}\right) \delta=7.75(\mathrm{~d}, J=8.2 \mathrm{~Hz}, 2 \mathrm{H}), 7.37-7.23(\mathrm{~m}, 3 \mathrm{H}), 7.06(\mathrm{~d}, J=7.7 \mathrm{~Hz}$, $1 \mathrm{H}), 7.00-6.90(\mathrm{~m}, 2 \mathrm{H}), 6.44(\mathrm{~d}, J=15.8 \mathrm{~Hz}, 1 \mathrm{H}), 6.01(\mathrm{dt}, J=15.8,6.6 \mathrm{~Hz}, 1 \mathrm{H}), 4.97(\mathrm{p}, J=6.8$ $\mathrm{Hz}, 1 \mathrm{H}), 4.72(\mathrm{dt}, J=6.5,2.5 \mathrm{~Hz}, 1 \mathrm{H}), 4.72(\mathrm{dt}, J=6.5,2.5 \mathrm{~Hz}, 2 \mathrm{H}), 4.03(\mathrm{~d}, J=6.7 \mathrm{~Hz}, 1 \mathrm{H}), 3.94$ - $3.88(\mathrm{~m}, 2 \mathrm{H}), 2.45(\mathrm{~s}, 3 \mathrm{H}) .{ }^{13} \mathrm{C}$ NMR (101 MHz, CDCl 3 ) $\delta=209.8(\mathrm{Cq}), 163.0(\mathrm{Cq}, \mathrm{d}, J=245.4$ $\mathrm{Hz}$ ), $143.4(\mathrm{Cq}), 138.6(\mathrm{Cq}, \mathrm{d}, J=7.9 \mathrm{~Hz}), 137.5(\mathrm{Cq}), 132.8$ (d, J = 2.3 Hz, CH), 130.0 (d, J = 8.5 $\mathrm{Hz}, \mathrm{CH}), 129.8(\mathrm{CH}), 127.24(\mathrm{CH}), 125.3(\mathrm{CH}), 122.3(\mathrm{~d}, J=2.9 \mathrm{~Hz}, \mathrm{CH}), 114.7(\mathrm{~d}, J=21.3 \mathrm{~Hz}$, $\mathrm{CH}), 112.9(\mathrm{~d}, \mathrm{~J}=22.0 \mathrm{~Hz}, \mathrm{CH}), 85.8(\mathrm{CH}), 76.2\left(\mathrm{CH}_{2}\right), 48.7\left(\mathrm{CH}_{2}\right), 46.0\left(\mathrm{CH}_{2}\right), 21.5\left(\mathrm{CH}_{3}\right) .{ }^{19} \mathrm{~F} \mathrm{NMR}$ (564 MHz, $\mathrm{CDCl}_{3}$ ) $\delta=-113.24$. (ESI)-HRMS calcd for $\mathrm{C}_{20} \mathrm{H}_{20} \mathrm{FNNaO}_{2} \mathrm{~S}[\mathrm{M}+\mathrm{Na}]^{+}$380.1091, found 380.1100 .

(E)-N-(buta-2,3-dien-1-yl)-N-(3-(3-cyanophenyl)allyl)-4-methylbenzenesulfonamide (1e)<smiles>C=CCN([125I])C/C=C/c1cccc(C#N)c1</smiles>

The general procedure (GP-1a) was followed using the correspondent enyne (245 mg, 0.7 $\mathrm{mmol})$. Purification by chromatography on silica gel yielded $1 \mathrm{e}(100 \mathrm{mg}, 39 \%)$ as a white solid. ${ }^{1} \mathrm{H}$ NMR $\left(400 \mathrm{MHz}, \mathrm{CDCl}_{3}\right) \delta=7.72(\mathrm{~d}, J=7.9 \mathrm{~Hz}, 2 \mathrm{H}), 7.51(\mathrm{~d}, J=8.3 \mathrm{~Hz}, 3 \mathrm{H}), 7.45-7.36(\mathrm{~m}$, $1 \mathrm{H}), 7.31(\mathrm{~d}, J=7.9 \mathrm{~Hz}, 2 \mathrm{H}), 6.44(\mathrm{~d}, J=15.9 \mathrm{~Hz}, 1 \mathrm{H}), 6.13-6.01(\mathrm{~m}, 1 \mathrm{H}), 4.94(\mathrm{p}, J=6.8 \mathrm{~Hz}$, $1 \mathrm{H}), 4.74-4.66(\mathrm{~m}, 2 \mathrm{H}), 4.01(\mathrm{~d}, J=6.5 \mathrm{~Hz}, 2 \mathrm{H}), 3.88$ (dd, J = 6.6, $3.1 \mathrm{~Hz}, 2 \mathrm{H}), 2.43(\mathrm{~s}, 3 \mathrm{H}) .{ }^{13} \mathrm{C}$ NMR (101 MHz, CDCl $\left.{ }_{3}\right) \delta=209.7(\mathrm{Cq}), 143.6(\mathrm{Cq}), 137.6(\mathrm{Cq}), 137.4(\mathrm{Cq}), 131.5(\mathrm{CH}), 131.1$ $(\mathrm{CH}), 130.5(\mathrm{CH}), 129.9(\mathrm{CH}), 129.8(\mathrm{CH}), 129.4(\mathrm{CH}), 127.2(\mathrm{CH}), 127.1(\mathrm{CH}), 118.6(\mathrm{Cq}), 112.9$ $(\mathrm{Cq}), 87.2(\mathrm{CH}), 76.3\left(\mathrm{CH}_{2}\right), 48.6\left(\mathrm{CH}_{2}\right), 46.3\left(\mathrm{CH}_{2}\right), 21.5\left(\mathrm{CH}_{3}\right)$. (ESI)-HRMS calcd for $\mathrm{C}_{21} \mathrm{H}_{20} \mathrm{~N}_{2} \mathrm{NaO}_{2} \mathrm{~S}[\mathrm{M}+\mathrm{Na}]^{+} 387.1138$ found 387.1131 . 
(E)-N-(buta-2,3-dien-1-yl)-4-methyl-N-(3-(m-tolyl)allyl)benzenesulfonamide (1f)<smiles>C=CCN(C)C/C=C/c1ccccc1C</smiles>

The general procedure (GP-1a) was followed using the correspondent enyne $(66.3 \mathrm{mg}, 0.19$ mmol). Purification by chromatography on silica gel yielded $\mathbf{1 f}(41.5 \mathrm{mg}, 63 \%)$ as a white solid. ${ }^{1} \mathrm{H}$ NMR $\left(400 \mathrm{MHz}, \mathrm{CDCl}_{3}\right) \delta=7.74(\mathrm{~d}, J=8.3 \mathrm{~Hz}, 2 \mathrm{H}), 7.34-7.24(\mathrm{~m}, 3 \mathrm{H}), 7.21-7.09(\mathrm{~m}, 3 \mathrm{H})$, $6.68(\mathrm{~d}, J=15.7 \mathrm{~Hz}, 1 \mathrm{H}), 5.86(\mathrm{dt}, J=15.7,6.8 \mathrm{~Hz}, 1 \mathrm{H}), 5.01-4.90(\mathrm{~m}, 1 \mathrm{H}), 4.71(\mathrm{dt}, J=6.7,2.5$ $\mathrm{Hz}, 2 \mathrm{H}), 4.03(\mathrm{~d}, J=6.9 \mathrm{~Hz}, 2 \mathrm{H}), 3.91(\mathrm{dt}, J=7.2,2.5 \mathrm{~Hz}, 2 \mathrm{H}), 2.43(\mathrm{~s}, 3 \mathrm{H}), 2.29(\mathrm{~s}, 3 \mathrm{H}) .{ }^{13} \mathrm{C}$ NMR $\left(101 \mathrm{MHz} \mathrm{CDCl}_{3}\right) \delta=207.8(\mathrm{Cq}), 143.4(\mathrm{Cq}), 137.6(\mathrm{Cq}), 135.4(\mathrm{Cq}), 135.4(\mathrm{CH}) 132.2(\mathrm{CH})$, $130.3(\mathrm{Cq}), 129.8(\mathrm{CH}), 127.8(\mathrm{CH}), 127.2(\mathrm{CH}), 126.1(\mathrm{CH}), 125.8(\mathrm{CH}), 125.0(\mathrm{CH}), 85.8(\mathrm{CH})$, $75.4\left(\mathrm{CH}_{2}\right), 49.0\left(\mathrm{CH}_{2}\right), 45.6\left(\mathrm{CH}_{2}\right), 21.5\left(\mathrm{CH}_{3}\right), 19.8\left(\mathrm{CH}_{3}\right)$. (ESI)-HRMS calcd for $\mathrm{C}_{21} \mathrm{H}_{23} \mathrm{NKO}_{2} \mathrm{~S}$ $[\mathrm{M}+\mathrm{K}]^{+}$392.1081, found 392.1074.

(E)-N-(buta-2,3-dien-1-yl)-N-(3-(4-methoxyphenyl)allyl)-4-methylbenzenesulfonamide (1g)<smiles>C=CCN([12CH3])C/C=C/c1ccc(OC)cc1</smiles>

The general procedure (GP-1a) was followed using the correspondent enyne (169 mg, 0.47 $\mathrm{mmol})$. Purification by chromatography on silica gel yielded $\mathbf{1 g}(127.5 \mathrm{mg}, 73 \%)$ as a white solid ${ }^{1} \mathrm{H}$ NMR $\left(400 \mathrm{MHz}, \mathrm{CDCl}_{3}\right) \delta=7.73(\mathrm{~d}, J=8.3 \mathrm{~Hz}, 2 \mathrm{H}), 7.30(\mathrm{~d}, J=7.7 \mathrm{~Hz}, 2 \mathrm{H}), 7.25-7.17$ $(\mathrm{m}, 2 \mathrm{H}), 6.89-6.78(\mathrm{~m}, 2 \mathrm{H}), 6.40(\mathrm{~d}, J=15.8 \mathrm{~Hz}, 1 \mathrm{H}), 5.84(\mathrm{dt}, J=15.8,6.8 \mathrm{~Hz}, 1 \mathrm{H}), 4.93(\mathrm{p}, J$ $=6.9 \mathrm{~Hz}, 1 \mathrm{H}), 4.69(\mathrm{dt}, J=6.6,2.5 \mathrm{~Hz}, 2 \mathrm{H}), 3.98(\mathrm{~d}, J=6.8 \mathrm{~Hz}, 2 \mathrm{H}), 3.89(\mathrm{dt}, J=7.1,2.5 \mathrm{~Hz}, 2 \mathrm{H})$, 3.80 (s, 3H), 2.42 (s, 3H). ${ }^{13} \mathrm{C}$ NMR (101 MHz, CDCl 3 ) $\delta=209.6(\mathrm{Cq}), 159.4(\mathrm{Cq}), 143.3(\mathrm{Cq})$, $137.6(\mathrm{Cq}), 133.7(\mathrm{CH}), 129.7(\mathrm{CH}), 129.0(\mathrm{Cq}), 127.7(\mathrm{CH}), 127.2(\mathrm{CH}), 121.3(\mathrm{CH}), 114.0(\mathrm{CH})$, $85.8\left(\mathrm{CH}_{2}\right), 76.2(\mathrm{CH}), 55.3\left(\mathrm{CH}_{3}\right), 49.0\left(\mathrm{CH}_{2}\right), 45.6\left(\mathrm{CH}_{2}\right), 21.6\left(\mathrm{CH}_{3}\right)$. (ESI)-HRMS calcd for $\mathrm{C}_{21} \mathrm{H}_{23} \mathrm{KNO}_{3} \mathrm{~S}[\mathrm{M}+\mathrm{K}]^{+}$408.1030, found 408.1042 .

(E)-N-(buta-2,3-dien-1-yl)-4-methyl-N-(3-(pyridin-3-yl)allyl)benzenesulfonamide (1h)<smiles>C=CCN([125I])C/C=C/c1cccnc1</smiles>

The general procedure (GP-1a) was followed using the correspondent enyne (200 mg, 0.61 mmol). Purification by chromatography on silica gel yielded $1 \mathrm{~h}(140 \mathrm{mg}, 67 \%)$ as a white solid. ${ }^{1} \mathrm{H}$ NMR $\left(400 \mathrm{MHz}, \mathrm{CDCl}_{3}\right) \delta=8.02-7.54(\mathrm{~m}, 3 \mathrm{H}), 7.30(\mathrm{~d}, J=8.0 \mathrm{~Hz}, 2 \mathrm{H}), 6.54(\mathrm{~d}, J=15.5 \mathrm{~Hz}$, $1 \mathrm{H}), 6.14-6.03(\mathrm{~m}, 1 \mathrm{H}), 4.93(\mathrm{p}, J=6.9 \mathrm{~Hz}, 1 \mathrm{H}), 4.69(\mathrm{dt}, J=6.6,2.4 \mathrm{~Hz}, 2 \mathrm{H}), 4.03(\mathrm{~d}, J=6.1$ $\mathrm{Hz}, 2 \mathrm{H}), 3.89(\mathrm{dt}, J=7.2,2.4 \mathrm{~Hz}, 2 \mathrm{H}), 2.42(\mathrm{~s}, 3 \mathrm{H}) .{ }^{13} \mathrm{C}$ NMR $\left(101 \mathrm{MHz}, \mathrm{CDCl}_{3}\right) \delta=209.6(\mathrm{Cq})$, $143.5(\mathrm{Cq}), 137.3(\mathrm{Cq}), 129.8(\mathrm{CH}), 127.2(\mathrm{CH}), 126.9(\mathrm{CH}), 85.7(\mathrm{CH}), 76.4\left(\mathrm{CH}_{2}\right), 48.8\left(\mathrm{CH}_{2}\right)$, $46.1\left(\mathrm{CH}_{2}\right), 21.6\left(\mathrm{CH}_{3}\right)$. Resonances of nuclei from the 3-pyridyl unit were almost undetectable 
even with long relaxation times. (ESI)-HRMS calcd for $\mathrm{C}_{19} \mathrm{H}_{21} \mathrm{~N}_{2} \mathrm{O}_{2} \mathrm{~S}[\mathrm{M}+\mathrm{H}]^{+} 341.1324$, found 341.1328 .

(E)-(3-(buta-2,3-dien-1-yloxy)prop-1-en-1-yl)benzene (1j)<smiles>C=CCCOC/C=C/c1ccccc1</smiles>

CuBr (103.8 mg, $0.72 \mathrm{mmol}$ ), paraformaldehyde (109 mg, $3.62 \mathrm{mmol}$ ), the 1,6-enyne ( $250 \mathrm{mg}$, $1.45 \mathrm{mmol}$ ) and dioxane $(5.3 \mathrm{~mL}$ ) were sequentially added to a $50 \mathrm{~mL}$ two-necked roundbottom flask equipped with a magnetic stirring. The resulting mixture was stirred at room temperature for 10 minutes prior to the before of $i \operatorname{Pr}_{2} \mathrm{NH}(410 \mu \mathrm{L}, 2.90 \mathrm{mmol})$. The mixture was then warmed to $110^{\circ} \mathrm{C}$ under air for 12 hours. The reaction mixture was cooled to room temperature and a saturated $\mathrm{NH}_{4} \mathrm{Cl}$ solution was added. The mixture was diluted with water and extracted with dietyl ether $(3 \times 10 \mathrm{~mL})$. The combined organic layers were dried over $\mathrm{Na}_{2} \mathrm{SO}_{4}$, filtered and concentred under reduced pressure. The resulting crude was purified by chromatography on silica gel ( $n$-hexane/EtOAc 97:3), which afforded $1 \mathbf{j}(120.1 \mathrm{mg}, 44 \%)$ as a yellow liquid. Spectroscopic data correspond to the literature. ${ }^{[6]}$

${ }^{1} \mathrm{H}$ NMR $\left(400 \mathrm{MHz}, \mathrm{CDCl}_{3}\right) \delta=7.50-7.20(\mathrm{~m}, 5 \mathrm{H}), 6.66(\mathrm{~d}, J=15.9 \mathrm{~Hz}, 1 \mathrm{H}), 6.34(\mathrm{dt}, J=15.9$, $6.1 \mathrm{~Hz}, 1 \mathrm{H}), 5.33(\mathrm{p}, J=6.8 \mathrm{~Hz}, 1 \mathrm{H}), 4.86(\mathrm{dt}, J=6.6,2.4 \mathrm{~Hz}, 2 \mathrm{H}), 4.22(\mathrm{dd}, J=6.1,1.5 \mathrm{~Hz}, 2 \mathrm{H})$, $4.12(\mathrm{dt}, J=7.0,2.5 \mathrm{~Hz}, 2 \mathrm{H}) .{ }^{13} \mathrm{C}$ NMR $\left(101 \mathrm{MHz} \mathrm{CDCl}_{3}\right) \delta=209.4(\mathrm{Cq}), 136.7(\mathrm{Cq}), 132.7(\mathrm{CH})$, $128.6(\mathrm{CH}), 127.7(\mathrm{CH}), 126.5(\mathrm{CH}), 125.8(\mathrm{CH}), 87.8(\mathrm{CH}), 75.8\left(\mathrm{CH}_{2}\right), 70.5\left(\mathrm{CH}_{2}\right), 67.9\left(\mathrm{CH}_{2}\right)$. HRMS calcd for $\mathrm{C}_{19} \mathrm{H}_{14} \mathrm{NaO}[\mathrm{M}+\mathrm{Na}]^{+}$209.0937, found 209.0926.

$\mathrm{N}$-cinnamyl-N-(3-cyclohexylideneallyl)-4-methylbenzenesulfonamide (1k)<smiles>[As]N(C/C=C/c1ccccc1)C/C=C/c1ccccc1</smiles>

CuBr (28.6 mg, $0.2 \mathrm{mmol})$, toluene $(2 \mathrm{~mL}), 4$-methyl- $\mathrm{N}$-(prop-2-yn-1-yl)benzenesulfonamide (420 mg, $2.0 \mathrm{mmol}$ ) and cyclohexanone $(239 \mathrm{mg}, 2.2 \mathrm{mmol})$ were sequentially added to a Schlenk tube kept under a nitrogen atmosphere. The resulting mixture was stirred at room temperature for 10 minutes prior to the addition of pyrrolidine ( $182 \mu \mathrm{L}, 2.2 \mathrm{mmol})$, and then heated at $100^{\circ} \mathrm{C}$. After 3 hours, the reaction mixture was cooled and filtered through a short pad of silica gel, eluting with acetone $(70 \mathrm{~mL})$. The crude product was then dissolved in toluene $(6 \mathrm{~mL})$ and transferred to a Schlenk tube containing $\mathrm{ZnI}_{2}$ (191.4 mg, $1.2 \mathrm{mmol}$ ). The tube was placed in a pre-heated oil bath at $120^{\circ} \mathrm{C}$ and kept under stirring for 7 hours. Upon complete conversion monitored by TLC, the crude reaction mixture was filtrated through a short pad of silica gel, eluting with acetone $(50 \mathrm{~mL})$. The resulting crude was purified by chromatography on silica gel (n-hexane/EtOAc 9:1), which afforded N-(3-cyclohexylideneallyl)-4methylbenzenesulfonamide (148.0 mg, $25 \%$ ) as a white solid. ${ }^{[7]} \mathrm{N}$-(3-cyclohexylideneallyl)-4methylbenzenesulfonamide was transferred in a Schlenk tube equipped with a magnetic 
stirring bar and $\mathrm{K}_{2} \mathrm{CO}_{3}(105 \mathrm{mg}, 0.76 \mathrm{mmol})$, acetone $(1.5 \mathrm{~mL})$ and cinnamyl chloride $(103 \mu \mathrm{L}$, $0.66 \mathrm{mmol}$ ) were then added sequentially. The reaction mixture was stirred at $60^{\circ} \mathrm{C}$ overnight. The mixture was cooled to room temperature, diluted with water and extracted with EtOAc $(3 \times 10 \mathrm{~mL})$. The combined organic layers were dried over $\mathrm{Na}_{2} \mathrm{SO}_{4}$, filtered and concentred under reduced pressure. The resulting crude was purified by chromatography on silica gel ( $n$ hexane/EtOAc 9:1), which afforded $\mathbf{1 k}(152 \mathrm{mg}, 70 \%)$ as a white solid. ${ }^{1} \mathbf{H}$ NMR (400 $\mathbf{~ M H z}$, $\left.\mathrm{CDCl}_{3}\right) \delta=7.74(\mathrm{~d}, J=8.2 \mathrm{~Hz}, 2 \mathrm{H}), 7.34-7.20(\mathrm{~m}, 7 \mathrm{H}), 6.44(\mathrm{~d}, J=15.8 \mathrm{~Hz}, 1 \mathrm{H}), 5.98(\mathrm{dt}, J=$ $15.8,6.7 \mathrm{~Hz}, 1 \mathrm{H}), 4.75(\mathrm{tt}, J=6.8,2.0 \mathrm{~Hz}, 1 \mathrm{H}), 4.03(\mathrm{dd}, J=6.5,1.3 \mathrm{~Hz}, 2 \mathrm{H}), 3.84(\mathrm{~d}, J=6.9 \mathrm{~Hz}$, 2H), 2.42 (s, 3H), $2.15-1.99(\mathrm{~m}, 4 \mathrm{H}), 1.60-1.46(\mathrm{~m}, 6 \mathrm{H}) .{ }^{13} \mathrm{C} \mathrm{NMR}\left(101 \mathrm{MHz}, \mathrm{CDCl}_{3}\right) \delta=200.2$ $(\mathrm{Cq}), 143.2(\mathrm{Cq}), 137.8(\mathrm{Cq}), 136.3(\mathrm{Cq}), 133.9(\mathrm{CH}), 129.7(\mathrm{CH}), 128.6(\mathrm{CH}), 127.9(\mathrm{CH}), 127.2$ $(\mathrm{CH}), 126.4(\mathrm{CH}), 123.9(\mathrm{CH}), 103.9(\mathrm{Cq}), 84.0(\mathrm{CH}), 48.3\left(\mathrm{CH}_{2}\right), 46.8\left(\mathrm{CH}_{2}\right), 31.3\left(\mathrm{CH}_{2}\right), 27.2$ $\left(\mathrm{CH}_{2}\right), 25.9\left(\mathrm{CH}_{2}\right), 21.6\left(\mathrm{CH}_{3}\right)$. (ESI)-HRMS calcd for $\mathrm{C}_{25} \mathrm{H}_{30} \mathrm{NO}_{2} \mathrm{~S}[\mathrm{M}+\mathrm{H}]^{+}$408.1992, found 408.2001.

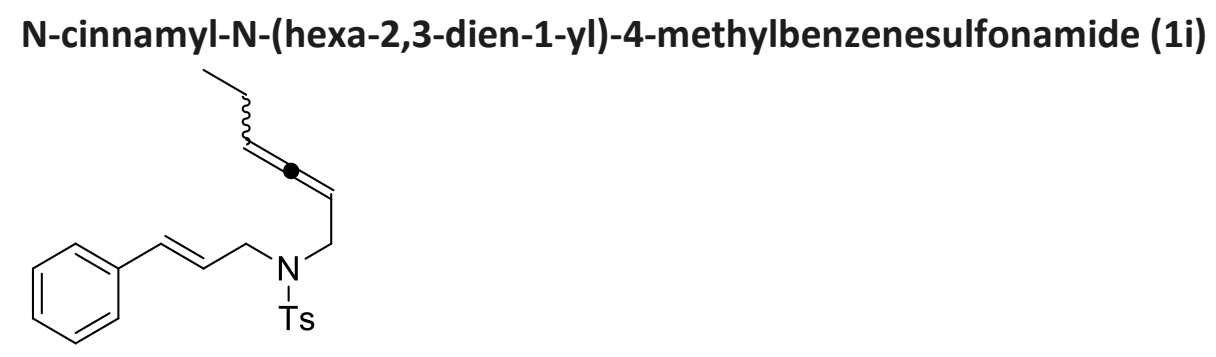

The general procedure (GP-1b) was followed using propionaldehyde. Purification by chromatography on silica gel yielded $\mathbf{1 i}(36.7 \mathrm{mg}, 5 \%)$ as a viscous oil. ${ }^{1} \mathbf{H}$ NMR $(400 \mathbf{~ M H z}$, acetone) $\delta=7.80(\mathrm{~d}, J=8.3 \mathrm{~Hz}, 2 \mathrm{H}), 7.44(\mathrm{dd}, J=8.6,0.8 \mathrm{~Hz}, 2 \mathrm{H}), 7.45-7.22(\mathrm{~m}, 5 \mathrm{H}), 6.56(\mathrm{~d}$, $J=15.9 \mathrm{~Hz}, 1 \mathrm{H}), 6.08(\mathrm{dt}, J=15.9,6.6 \mathrm{~Hz}, 1 \mathrm{H}), 5.31-5.21(\mathrm{~m}, 1 \mathrm{H}), 5.05-4.94(\mathrm{~m}, 1 \mathrm{H}), 4.05(\mathrm{~d}$, $J=6.9 \mathrm{~Hz}, 2 \mathrm{H}), 3.89(\mathrm{dd}, J=6.9,2.3 \mathrm{~Hz}, 2 \mathrm{H}), 2.45(\mathrm{~s}, 3 \mathrm{H}), 2.04-1.92(\mathrm{~m}, 2 \mathrm{H}), 0.96(\mathrm{t}, J=7.4$ $\mathrm{Hz}, 3 \mathrm{H}) .{ }^{13} \mathrm{C}$ NMR (101 MHz, acetone) $\delta=205.6(\mathrm{Cq}), 144.2(\mathrm{Cq}), 138.9(\mathrm{Cq}), 137.4(\mathrm{Cq}), 134.5$ $(\mathrm{CH}), 130.6(\mathrm{CH}), 129.4(\mathrm{CH}), 128.6(\mathrm{CH}), 128.1(\mathrm{CH}), 127.3(\mathrm{CH}), 124.9(\mathrm{CH}), 94.8(\mathrm{CH}), 87.9$ $\left(\mathrm{CH}_{2}\right), 49.5\left(\mathrm{CH}_{2}\right), 47.3\left(\mathrm{CH}_{2}\right), 22.3\left(\mathrm{CH}_{2}\right), 21.4\left(\mathrm{CH}_{3}\right), 13.8\left(\mathrm{CH}_{3}\right)$. (ESI)-HRMS calcd for $\mathrm{C}_{22} \mathrm{H}_{25} \mathrm{NNaO}_{2} \mathrm{~S}[\mathrm{M}+\mathrm{Na}]^{+} 390.1498$, found 390.1503 .

N-cinnamyl-4-methyl-N-(4-phenylbuta-2,3-dien-1-yl)benzenesulfonamide (1I)<smiles>CN(/C=C/c1ccccc1)C/C=C/c1ccccc1</smiles>

The general procedure (GP-1b) was followed using benzaldehyde. Purification by chromatography on silica gel yielded 11 (91.0 mg, 22\%) as a white solid. ${ }^{1} \mathbf{H}$ NMR (400 $\mathbf{~ M H z}$, $\left.\mathrm{CDCl}_{3}\right) \delta=7.78(\mathrm{~d}, J=8.3 \mathrm{~Hz}, 2 \mathrm{H}), 7.41-7.07(\mathrm{~m}, 12 \mathrm{H}), 6.39(\mathrm{~d}, J=15.9 \mathrm{~Hz}, 1 \mathrm{H}), 6.15(\mathrm{dt}, J=$ $6.4,2.3 \mathrm{~Hz}, 1 \mathrm{H}), 5.99(\mathrm{dt}, J=15.8,6.8 \mathrm{~Hz}, 1 \mathrm{H}), 5.42(\mathrm{q}, J=6.7 \mathrm{~Hz}, 1 \mathrm{H}), 4.20-3.92(\mathrm{~m}, 4 \mathrm{H}), 2.45$ (s, 3H). ${ }^{13} \mathrm{C}$ NMR (101 MHz, CDCl $) \delta=206.4(\mathrm{Cq}), 143.4(\mathrm{Cq}), 137.5(\mathrm{Cq}), 136.1(\mathrm{Cq}), 134.3$ $(\mathrm{CH}), 133.5(\mathrm{Cq}), 129.8(\mathrm{CH}), 128.7(\mathrm{CH}), 128.5(\mathrm{CH}), 127.9(\mathrm{CH}), 127.4(\mathrm{CH}), 127.3(\mathrm{CH}), 126.9$ 
$(\mathrm{CH}), 126.4(\mathrm{CH}), 123.4(\mathrm{CH}), 96.0(\mathrm{CH}), 90.6(\mathrm{CH}), 49.0\left(\mathrm{CH}_{2}\right), 45.8\left(\mathrm{CH}_{2}\right), 21.5\left(\mathrm{CH}_{3}\right)$. (ESI)HRMS calcd for $\mathrm{C}_{26} \mathrm{H}_{25} \mathrm{KNO}_{2} \mathrm{~S}[\mathrm{M}+\mathrm{K}]^{+} 454.1238$, found 453.1223 .

N-cinnamyl-4-methyl-N-(propa-1,2-dien-1-yl)benzenesulfonamide (3a)<smiles>C=CN([As])C/C=C/c1ccccc1</smiles>

The general procedure (GP-2) was followed using the correspondent enyne (828 mg, 2.54 $\mathrm{mmol})$. Purification by chromatography on silica gel yielded $3 a(560 \mathrm{mg}, 67 \%)$ as a white solid. ${ }^{1} \mathrm{H}$ NMR $\left(400 \mathrm{MHz}^{\mathrm{CDCl}}{ }_{3}\right) \delta=7.72(\mathrm{~d}, J=8.2 \mathrm{~Hz}, 2 \mathrm{H}), 7.38-7.20(\mathrm{~m}, 7 \mathrm{H}), 6.89(\mathrm{t}, J=6.2 \mathrm{~Hz}$, $1 \mathrm{H}), 6.45(\mathrm{~d}, J=15.9 \mathrm{~Hz}, 1 \mathrm{H}), 5.98(\mathrm{dt}, J=15.9,6.2 \mathrm{~Hz}, 1 \mathrm{H}), 5.31(\mathrm{~d}, J=6.2 \mathrm{~Hz}, 2 \mathrm{H}), 3.98$ (dd, $J$ $=6.2,1.4 \mathrm{~Hz}, 2 \mathrm{H}), 2.40(\mathrm{~s}, 3 \mathrm{H}) \cdot{ }^{13} \mathrm{C} \mathrm{NMR}\left(101 \mathrm{MHz}, \mathrm{CDCl}_{3}\right) \delta=201.9(\mathrm{Cq}), 143.8(\mathrm{Cq}), 136.5(\mathrm{Cq})$, $135.7(\mathrm{Cq}), 133.6(\mathrm{CH}), 129.8(\mathrm{CH}), 128.5(\mathrm{CH}), 127.8(\mathrm{CH}), 127.3(\mathrm{CH}), 126.4(\mathrm{CH}), 123.5(\mathrm{CH})$, $100.1(\mathrm{CH}), 88.0\left(\mathrm{CH}_{2}\right), 48.6\left(\mathrm{CH}_{2}\right), 21.6\left(\mathrm{CH}_{3}\right)$. (ESI)-HRMS calcd for $\mathrm{C}_{19} \mathrm{H}_{19} \mathrm{NNaO}_{2} \mathrm{~S}[\mathrm{M}+\mathrm{Na}]^{+}$ 348.1029 , found 347.1017.

(E)-4-methyl-N-(propa-1,2-dien-1-yl)-N-(3-(o-tolyl)allyl)benzenesulfonamide (3b)<smiles>C=C=CN([AsH3-])C/C=C/c1ccccc1C</smiles>

The general procedure (GP-2) was followed using the correspondent enyne (140.0 mg, 0.47 $\mathrm{mmol})$. Purification by chromatography on silica gel yielded $3 \mathbf{b}(78.7 \mathrm{mg}, 56 \%)$ as a viscous clear oil. ${ }^{1} \mathrm{H}$ NMR $(400 \mathrm{MHz}$, acetone) $\delta=7.80(\mathrm{~d}, J=8.2 \mathrm{~Hz}, 2 \mathrm{H}), 7.45(\mathrm{~d}, J=8.1 \mathrm{~Hz}, 2 \mathrm{H}), 7.35$ $-7.27(\mathrm{~m}, 1 \mathrm{H}), 7.18-7.09(\mathrm{~m}, 3 \mathrm{H}), 6.92(\mathrm{t}, J=6.3 \mathrm{~Hz}, 1 \mathrm{H}), 6.78(\mathrm{~d}, J=15.8 \mathrm{~Hz}, 1 \mathrm{H}), 5.92(\mathrm{dt}, J$ $=15.8,6.1 \mathrm{~Hz}, 1 \mathrm{H}), 5.41(\mathrm{~d}, J=6.3 \mathrm{~Hz}, 2 \mathrm{H}), 4.05(\mathrm{dd}, J=6.1,1.5 \mathrm{~Hz}, 2 \mathrm{H}), 2.44(\mathrm{~s}, 3 \mathrm{H}), 2.30(\mathrm{~s}$, 3H). ${ }^{13} \mathrm{C} \mathrm{NMR}$ (101 MHz, acetone) $\delta=201.9(\mathrm{Cq}), 144.0(\mathrm{Cq}), 136.1(\mathrm{Cq}), 135.6(\mathrm{Cq}), 135.2(\mathrm{Cq})$, $131.1(\mathrm{CH}), 130.1(\mathrm{CH}), 129.8(\mathrm{CH}), 127.5(\mathrm{CH}), 127.3(\mathrm{CH}), 126.02(\mathrm{CH}), 125.6(\mathrm{CH}), 125.0(\mathrm{CH})$, $99.8(\mathrm{CH}), 87.3\left(\mathrm{CH}_{2}\right), 48.6\left(\mathrm{CH}_{2}\right), 20.5\left(\mathrm{CH}_{3}\right), 18.8\left(\mathrm{CH}_{3}\right)$. (ESI)-HRMS calcd for $\mathrm{C}_{20} \mathrm{H}_{22} \mathrm{NO}_{2} \mathrm{~S}$ $[\mathrm{M}+\mathrm{H}]^{+} 340.1366$, found 340.1378 .

(E)-N-(3-(4-methoxyphenyl)allyl)-4-methyl-N-(propa-1,2-dien-1-yl)benzenesulfonamide (3c)<smiles>C=CN([AsH3])C/C=C/c1ccc(OC)cc1</smiles>

The general procedure (GP-2) was followed using the correspondent enyne (78.6 mg, 0.22 mmol). Purification by chromatography on silica gel yielded $3 \mathrm{c}(38.6 \mathrm{mg}, 49 \%)$ as a viscous oil. ${ }^{1} \mathrm{H}$ NMR (400 MHz, Acetone) $\delta=7.78(\mathrm{~d}, J=8.3 \mathrm{~Hz}, 2 \mathrm{H}), 7.49-7.40(\mathrm{~m}, 2 \mathrm{H}), 7.29$ (d, $J=8.7$ $\mathrm{Hz}, 2 \mathrm{H}), 6.95-6.83(\mathrm{~m}, 3 \mathrm{H}), 6.47(\mathrm{~d}, J=15.9 \mathrm{~Hz}, 1 \mathrm{H}), 5.91(\mathrm{dt}, J=15.9,6.3 \mathrm{~Hz}, 1 \mathrm{H}), 5.38(\mathrm{~d}, J=$ $6.3 \mathrm{~Hz}, 2 \mathrm{H}), 3.98(\mathrm{~d}, J=6.3 \mathrm{~Hz}, 2 \mathrm{H}), 3.80(\mathrm{~s}, 3 \mathrm{H}), 2.43(\mathrm{~s}, 3 \mathrm{H}) .{ }^{13} \mathrm{C} \mathrm{NMR}\left(101 \mathrm{MHz}, \mathrm{CDCl}_{3}\right) \delta=$ $207.1(\mathrm{Cq}), 164.8(\mathrm{Cq}), 149.2(\mathrm{Cq}), 141.2(\mathrm{Cq}), 138.3(\mathrm{CH}), 135.0(\mathrm{CH}), 134.4(\mathrm{Cq}), 132.8(\mathrm{CH})$, $132.5(\mathrm{CH}), 126.2(\mathrm{CH}), 119.1(\mathrm{CH}), 104.9(\mathrm{CH}), 92.6\left(\mathrm{CH}_{2}\right), 59.9\left(\mathrm{CH}_{2}\right), 53.7\left(\mathrm{CH}_{3}\right), 25.8\left(\mathrm{CH}_{3}\right)$. (ESI)-HRMS calcd for $\mathrm{C}_{20} \mathrm{H}_{21} \mathrm{KNO}_{3} \mathrm{~S}[\mathrm{M}+\mathrm{K}]^{+} 394.0874$ found 394.0881. 
(E)-N-(3-(4-chlorophenyl)allyl)-4-methyl-N-(propa-1,2-dien-1-yl)benzenesulfonamide (3d)<smiles>C=CN([135I])C/C=C/c1ccc(Cl)cc1</smiles>

The general procedure (GP-2) was followed using the correspondent enyne (300.0 mg, 0.83 mmol). Purification by chromatography on silica gel yielded $\mathbf{3 d}(111.2 \mathrm{mg}, 37 \%)$ as a white solid. ${ }^{1} \mathrm{H}$ NMR $\left(300 \mathrm{MHz}, \mathrm{CDCl}_{3}\right) \delta=7.71(\mathrm{~d}, J=8.3 \mathrm{~Hz}, 2 \mathrm{H}), 7.45-7.02(\mathrm{~m}, 6 \mathrm{H}), 6.88(\mathrm{t}, J=6.2$ $\mathrm{Hz}, 1 \mathrm{H}), 6.40(\mathrm{~d}, J=16.0 \mathrm{~Hz}, 1 \mathrm{H}), 5.95(\mathrm{dt}, J=15.9,6.1 \mathrm{~Hz}, 1 \mathrm{H}), 5.30(\mathrm{~d}, J=6.2 \mathrm{~Hz}, 2 \mathrm{H}), 3.95(\mathrm{~d}$, $J=6.5 \mathrm{~Hz}, 2 \mathrm{H}), 2.41(\mathrm{~s}, 3 \mathrm{H}) .{ }^{13} \mathrm{C} \mathrm{NMR}\left(75 \mathrm{MHz}, \mathrm{CDCl}_{3}\right) \delta=201.8(\mathrm{Cq}), 143.8(\mathrm{Cq}), 135.7(\mathrm{Cq})$, $135.0(\mathrm{Cq}), 133.4(\mathrm{Cq}), 132.1(\mathrm{CH}), 129.7(\mathrm{CH}), 128.6(\mathrm{CH}), 127.5(\mathrm{CH}), 127.3(\mathrm{CH}), 124.2(\mathrm{CH})$, $100.1(\mathrm{CH}), 87.9\left(\mathrm{CH}_{2}\right), 48.4\left(\mathrm{CH}_{2}\right), 21.5\left(\mathrm{CH}_{3}\right)$. (ESI)-HRMS calcd for $\mathrm{C}_{19} \mathrm{H}_{19} \mathrm{ClNO}_{2} \mathrm{~S}[\mathrm{M}+\mathrm{H}]^{+}$ 360.0820 , found 360.0811 .

(E)-N-(3-(3-cyanophenyl)allyl)-4-methyl-N-(propa-1,2-dien-1-yl)benzenesulfonamide (3e)<smiles>C=CCN([AsH3])C/C=C/c1cccc(C#N)c1</smiles>

The general procedure (GP-2) was followed using the correspondent enyne (200.0 mg, 0.57 $\mathrm{mmol})$. Purification by chromatography on silica gel yielded $3 \mathrm{e}(54.3 \mathrm{mg}, 27 \%)$ as a viscous yellow oil. ${ }^{1} \mathrm{H}$ NMR $(400 \mathrm{MHz}$, acetone) $\delta=7.79(\mathrm{~d}, J=8.0 \mathrm{~Hz}, 2 \mathrm{H}), 7.73-7.61(\mathrm{~m}, 3 \mathrm{H}), 7.54(\mathrm{t}$, $J=7.7 \mathrm{~Hz}, 1 \mathrm{H}), 7.43(\mathrm{~d}, J=8.0 \mathrm{~Hz}, 2 \mathrm{H}), 6.93(\mathrm{t}, J=6.3 \mathrm{~Hz}, 1 \mathrm{H}), 6.58(\mathrm{~d}, J=16.0 \mathrm{~Hz}, 1 \mathrm{H}), 6.23(\mathrm{dt}$, $J=16.1,6.0 \mathrm{~Hz}, 1 \mathrm{H}), 5.40(\mathrm{~d}, J=6.2 \mathrm{~Hz}, 2 \mathrm{H}), 4.06(\mathrm{~d}, J=5.7 \mathrm{~Hz}, 2 \mathrm{H}), 2.42(\mathrm{~s}, 3 \mathrm{H}) .{ }^{13} \mathrm{C}$ NMR $(101$ $\mathrm{MHz}$, acetone) $\delta=201.8(\mathrm{Cq}), 144.1(\mathrm{Cq}), 138.0(\mathrm{Cq}), 136.0(\mathrm{Cq}), 131.0(\mathrm{CH}), 130.9(\mathrm{CH}), 130.6$ $(\mathrm{CH}), 129.8(\mathrm{CH}), 129.7(\mathrm{CH}), 129.7(\mathrm{CH}), 127.3(\mathrm{CH}), 126.7(\mathrm{CH}), 118.3(\mathrm{Cq}), 112.7(\mathrm{Cq}), 99.8$ (CH), $87.6\left(\mathrm{CH}_{2}\right), 48.1\left(\mathrm{CH}_{2}\right), 20.6\left(\mathrm{CH}_{3}\right)$. (ESI)-HRMS calcd for $\mathrm{C}_{20} \mathrm{H}_{18} \mathrm{~N}_{2} \mathrm{NaO}_{2} \mathrm{~S}[\mathrm{M}+\mathrm{Na}]^{+}$ 373.0981, found 373.0992 .

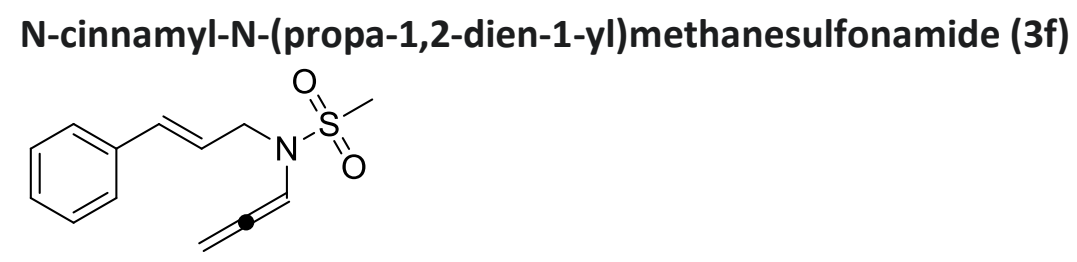

The general procedure (GP-2) was followed using the correspondent enyne ( $250 \mathrm{mg}, 1.00$ $\mathrm{mmol})$. Purification by chromatography on silica gel yielded $\mathbf{3 f}(124.5 \mathrm{mg}, 50 \%)$ as a white solid. ${ }^{1} \mathrm{H}$ NMR $\left(400 \mathrm{MHz} \mathrm{CDCl}_{3}\right) \delta=7.40-7.22(\mathrm{~m}, 5 \mathrm{H}), 6.73(\mathrm{tt}, J=6.2,0.7 \mathrm{~Hz}, 1 \mathrm{H}), 6.63(\mathrm{~d}, J$ $=15.8 \mathrm{~Hz}, 1 \mathrm{H}), 6.18(\mathrm{dt}, J=15.9,6.5 \mathrm{~Hz}, 1 \mathrm{H}), 5.44(\mathrm{~d}, J=6.3 \mathrm{~Hz}, 2 \mathrm{H}), 4.15$ (ddd, $J=6.4,1.4,0.7$ $\mathrm{Hz}, 2 \mathrm{H}), 2.93(\mathrm{~s}, 3 \mathrm{H}) .{ }^{13} \mathrm{C}$ NMR (101 MHz, CDCl 3 ) $\delta=201.3(\mathrm{Cq}), 136.2(\mathrm{Cq}), 134.3(\mathrm{CH}), 128.7$ $(\mathrm{CH}), 128.1(\mathrm{CH}), 126.5(\mathrm{CH}), 123.0(\mathrm{CH}), 99.7(\mathrm{CH}), 88.2\left(\mathrm{CH}_{2}\right), 48.1\left(\mathrm{CH}_{2}\right), 39.1\left(\mathrm{CH}_{3}\right)$. (ESI)HRMS calcd for $\mathrm{C}_{13} \mathrm{H}_{16} \mathrm{NO}_{2} \mathrm{~S}[\mathrm{M}+\mathrm{H}]^{+} 250.0896$, found 250.0910 . 
(E)-N-(propa-1,2-dien-1-yl)-N-(3-(o-tolyl)allyl)methanesulfonamide (3g)<smiles>C=C=CN(C/C=C/c1ccccc1C)S(C)(=O)=O</smiles>

The general procedure (GP-2) was followed using the correspondent enyne (175 mg, 0.67 $\mathrm{mmol})$. Purification by chromatography on silica gel yielded $3 \mathrm{~g}(43.7 \mathrm{mg}, 25.0 \%)$ as a pale yellow solid. ${ }^{1} \mathrm{H}$ NMR $(400 \mathrm{MHz}$, acetone) $\delta 7.51-7.43(\mathrm{~m}, 1 \mathrm{H}), 7.20-7.11(\mathrm{~m}, 3 \mathrm{H}), 6.88$ (dd, $J=15.8,1.6 \mathrm{~Hz}, 1 \mathrm{H}), 6.76(\mathrm{t}, J=6.3 \mathrm{~Hz}, 1 \mathrm{H}), 6.13(\mathrm{dt}, J=15.7,6.2 \mathrm{~Hz}, 1 \mathrm{H}), 5.49(\mathrm{~d}, J=6.3 \mathrm{~Hz}$, $2 \mathrm{H}), 4.18(\mathrm{~d}, J=6.2 \mathrm{~Hz}, 2 \mathrm{H}), 3.04(\mathrm{~s}, 3 \mathrm{H}), 2.33(\mathrm{~s}, 3 \mathrm{H}) .{ }^{13} \mathrm{C} \mathrm{NMR}(101 \mathrm{MHz}$, acetone) $\delta=201.4$ $(\mathrm{Cq}), 135.6(\mathrm{Cq}), 135.3(\mathrm{Cq}), 131.4(\mathrm{CH}), 130.2(\mathrm{CH}), 127.6(\mathrm{CH}), 126.1(\mathrm{CH}), 125.6(\mathrm{CH}), 125.3$ $(\mathrm{CH}), 99.6(\mathrm{CH}), 87.4\left(\mathrm{CH}_{2}\right), 48.5\left(\mathrm{CH}_{2}\right), 38.0\left(\mathrm{CH}_{3}\right), 18.9\left(\mathrm{CH}_{3}\right)$. (ESI)-HRMS calcd for $\mathrm{C}_{14} \mathrm{H}_{17} \mathrm{NNaO}_{2} \mathrm{~S}[\mathrm{M}+\mathrm{Na}]^{+} 286.0872$, found 286.0864 .

$\mathrm{N}$-cinnamyl-N-(propa-1,2-dien-1-yl)benzenesulfonamide (3h)<smiles>C=CCN(C/C=C/c1ccccc1)S(=O)(=O)c1ccccc1</smiles>

The general procedure (GP-2) was followed using the correspondent enyne (370 mg, 1.19 $\mathrm{mmol})$. Purification by chromatography on silica gel yielded $3 \mathrm{~h}(252.2 \mathrm{mg}, 68 \%)$ as a pale white solid. ${ }^{1} \mathrm{H}$ NMR (400 MHz, acetone) $\delta=7.93(\mathrm{~d}, J=7.6 \mathrm{~Hz}, 2 \mathrm{H}), 7.82-7.56(\mathrm{~m}, 3 \mathrm{H}), 7.40-7.22$ $(\mathrm{m}, 5 \mathrm{H}), 6.91(\mathrm{t}, J=6.3 \mathrm{~Hz}, 1 \mathrm{H}), 6.55(\mathrm{~d}, J=15.9 \mathrm{~Hz}, 1 \mathrm{H}), 6.09(\mathrm{dt}, J=15.9,6.1 \mathrm{~Hz}, 1 \mathrm{H}), 5.39(\mathrm{~d}$, $J=6.2 \mathrm{~Hz}, 2 \mathrm{H}), 4.04(\mathrm{~d}, J=6.1 \mathrm{~Hz}, 2 \mathrm{H}) .{ }^{13} \mathrm{C}$ NMR $(101 \mathrm{MHz}$, acetone) $\delta=201.9(\mathrm{Cq}), 138.8(\mathrm{Cq})$, $136.6(\mathrm{Cq}), 133.4(\mathrm{CH}), 133.1(\mathrm{CH}), 129.3(\mathrm{CH}), 128.5(\mathrm{CH}), 127.7(\mathrm{CH}), 127.2(\mathrm{CH}), 126.3(\mathrm{CH})$, $123.6(\mathrm{CH}), 99.7(\mathrm{CH}), 87.4\left(\mathrm{CH}_{2}\right), 48.5\left(\mathrm{CH}_{2}\right)$. (ESI)-HRMS calcd for $\mathrm{C}_{18} \mathrm{H}_{18} \mathrm{NO}_{2} \mathrm{~S}[\mathrm{M}+\mathrm{H}]^{+}$ 312.1053, found 312.1040.

$\mathrm{N}$-cinnamyl-2,4,6-trimethyl-N-(propa-1,2-dien-1-yl)benzenesulfonamide (3j)<smiles>C=C=CN(C/C=C/c1ccccc1)S(=O)(=O)c1c(C)cc(C)cc1C</smiles>

The general procedure (GP-2) was followed using the correspondent enyne ( $270 \mathrm{mg}, 0.76$ mmol). Purification by chromatography on silica gel yielded $\mathbf{3 j}$ (108.2 $\mathrm{mg}, 40 \%)$ as a viscous liquid. ${ }^{1} \mathrm{H}$ NMR $\left(300 \mathrm{MHz}, \mathrm{CDCl}_{3}\right) \delta=7.34-7.16(\mathrm{~m}, 5 \mathrm{H}), 6.94(\mathrm{~s}, 2 \mathrm{H}), 6.89(\mathrm{t}, J=6.3 \mathrm{~Hz}, 1 \mathrm{H})$, $6.30(\mathrm{~d}, J=15.9 \mathrm{~Hz}, 1 \mathrm{H}), 5.94(\mathrm{dt}, J=15.9,6.4 \mathrm{~Hz}, 1 \mathrm{H}), 5.36(\mathrm{~d}, J=6.3 \mathrm{~Hz}, 2 \mathrm{H}), 3.96(\mathrm{~d}, J=6.3$ $\mathrm{Hz}, 2 \mathrm{H}), 2.64(\mathrm{~s}, 6 \mathrm{H}), 2.26(\mathrm{~s}, 3 \mathrm{H}) .{ }^{13} \mathrm{C}$ NMR $(75 \mathrm{MHz}$, acetone) $\delta=200.7(\mathrm{Cq}), 143.2(\mathrm{Cq}), 139.9$ $(\mathrm{CH}), 136.6(\mathrm{Cq}), 133.4(\mathrm{CH}), 133.2(\mathrm{Cq}), 132.1(\mathrm{CH}), 128.5(\mathrm{CH}), 127.6(\mathrm{CH}), 126.2(\mathrm{CH}), 123.2$ (CH), $99.5(\mathrm{CH}), 87.7\left(\mathrm{CH}_{2}\right), 47.4\left(\mathrm{CH}_{2}\right), 22.3\left(\mathrm{CH}_{3}\right), 20.0\left(\mathrm{CH}_{3}\right)$. (ESI)-HRMS calcd for $\mathrm{C}_{21} \mathrm{H}_{23} \mathrm{NKO}_{2} \mathrm{~S}$ $[\mathrm{M}+\mathrm{H}]^{+}$392.1081, found 392.1094. 
<smiles>C=C=CN(C/C=C/c1ccccc1)S(=O)(=O)c1ccc([N+](=O)[O-])cc1</smiles>

The general procedure (GP-2) was followed using the correspondent enyne (357.0 mg, 1.00 mmol). Purification by chromatography on silica gel yielded $3 \mathbf{k}(89.6 \mathrm{mg}$. $25 \%$ ) as a viscous yellow oil. ${ }^{1} \mathrm{H}$ NMR (300 MHz, acetone) $\delta=8.33(\mathrm{~d}, J=8.8 \mathrm{~Hz}, 2 \mathrm{H}), 8.02(\mathrm{~d}, J=8.8 \mathrm{~Hz}, 2 \mathrm{H}), 7.40$ $-7.22(\mathrm{~m}, 5 \mathrm{H}), 6.88(\mathrm{t}, J=6.2 \mathrm{~Hz}, 1 \mathrm{H}), 6.49(\mathrm{~d}, J=15.9 \mathrm{~Hz}, 1 \mathrm{H}), 5.95(\mathrm{dt}, J=15.8,6.3 \mathrm{~Hz}, 1 \mathrm{H})$, $5.40(\mathrm{~d}, J=6.2 \mathrm{~Hz}, 2 \mathrm{H}), 4.07(\mathrm{~d}, J=6.4 \mathrm{~Hz}, 2 \mathrm{H}) .{ }^{13} \mathrm{C} \mathrm{NMR}\left(75 \mathrm{MHz}, \mathrm{CDCl}_{3}\right) \delta=201.8(\mathrm{Cq}), 150.1$ (Cq), $144.4(\mathrm{Cq}), 135.9(\mathrm{Cq}), 134.5(\mathrm{CH}), 128.7(\mathrm{CH}), 128.5(\mathrm{CH}), 128.2(\mathrm{CH}), 126.3(\mathrm{CH}), 124.3$ (CH), $122.3(\mathrm{CH}), 99.4(\mathrm{CH}), 88.5\left(\mathrm{CH}_{2}\right), 49.1\left(\mathrm{CH}_{2}\right)$. (ESI)-HRMS calcd for $\mathrm{C}_{18} \mathrm{H}_{17} \mathrm{~N}_{2} \mathrm{O}_{4} \mathrm{~S}[\mathrm{M}+\mathrm{H}]^{+}$ 357.0904 , found 357.0894 .

\section{4-chloro-N-cinnamyl-N-(propa-1,2-dien-1-yl)benzenesulfonamide (3i)}<smiles>C=CCN(C/C=C/c1ccccc1)S(=O)(=O)c1ccc(Cl)cc1</smiles>

The general procedure (GP-2) was followed using the correspondent enyne ( $250 \mathrm{mg}, 0.72$ mmol). Purification by chromatography on silica gel yielded $3 \mathbf{i}(168 \mathrm{mg}, 67 \%)$ as a white solid. ${ }^{1} \mathrm{H}$ NMR (300 MHz, acetone) $\delta=7.91(\mathrm{~d}, J=8.6 \mathrm{~Hz}, 2 \mathrm{H}), 7.65(\mathrm{~d}, J=8.6 \mathrm{~Hz}, 2 \mathrm{H}), 7.48-7.18$ $(\mathrm{m}, 5 \mathrm{H}), 6.89(\mathrm{t}, J=6.3 \mathrm{~Hz}, 1 \mathrm{H}), 6.54(\mathrm{~d}, J=16.0 \mathrm{~Hz}, 1 \mathrm{H}), 6.07(\mathrm{dt}, J=15.9,6.2 \mathrm{~Hz}, 1 \mathrm{H}), 5.41(\mathrm{~d}$, $J=6.3 \mathrm{~Hz}, 2 \mathrm{H}), 4.04(\mathrm{~d}, J=6.2 \mathrm{~Hz}, 2 \mathrm{H}) .{ }^{13} \mathrm{C}$ NMR $(75 \mathrm{MHz}$, acetone) $\delta=202.0(\mathrm{Cq}), 138.9(\mathrm{Cq})$, $137.5(\mathrm{Cq}), 136.5(\mathrm{Cq}), 133.6(\mathrm{CH}), 129.5(\mathrm{CH}), 129.1(\mathrm{CH}), 128.5(\mathrm{CH}), 127.8(\mathrm{CH}), 126.3(\mathrm{CH})$, $123.2(\mathrm{CH}), 99.4\left(\mathrm{CH}_{2}\right), 87.6(\mathrm{CH}), 48.6\left(\mathrm{CH}_{2}\right)$. (ESI)-HRMS calcd for $\mathrm{C}_{18} \mathrm{H}_{17} \mathrm{CINO}_{2} \mathrm{~S}[\mathrm{M}+\mathrm{H}]^{+}$ 346.0663 , found 346.0670 .

$\mathrm{N}$-cinnamyl-N-(propa-1,2-dien-1-yl)naphthalene-1-sulfonamide (3l)<smiles>C=CCN(C/C=C/c1ccccc1)S(=O)(=O)c1cccc2ccccc12</smiles>

The general procedure (GP-2) was followed using the correspondent enyne (135 mg, 0.37 mmol). Purification by chromatography on silica gel yielded 31 (106 $\mathrm{mg}, 78 \%)$ as a viscous liquid. ${ }^{1} \mathbf{H}$ NMR (300 MHz, acetone) $\delta=8.57-8.51(\mathrm{~m}, 1 \mathrm{H}), 8.17-8.08(\mathrm{~m}, 2 \mathrm{H}), 8.07-8.00$ (m, $1 \mathrm{H}), 7.90(\mathrm{dd}, J=8.7,2.0 \mathrm{~Hz}, 1 \mathrm{H}), 7.77-7.72(\mathrm{~m}, 2 \mathrm{H}), 7.33-7.14(\mathrm{~m}, 5 \mathrm{H}), 6.98(\mathrm{t}, J=6.3$ $\mathrm{Hz}, 1 \mathrm{H}), 6.54(\mathrm{dt}, J=15.8,1.5 \mathrm{~Hz}, 1 \mathrm{H}), 6.07(\mathrm{dt}, J=15.9,6.2 \mathrm{~Hz}, 1 \mathrm{H}), 5.36(\mathrm{~d}, J=6.3 \mathrm{~Hz}, 2 \mathrm{H})$, $4.08(\mathrm{~d}, J=6.2 \mathrm{~Hz}, 2 \mathrm{H}) .{ }^{13} \mathrm{C}$ NMR (75 MHz, acetone) $\delta=201.9(\mathrm{Cq}), 136.6(\mathrm{Cq}), 135.9(\mathrm{Cq})$, $135.0(\mathrm{Cq}), 133.4(\mathrm{CH}), 132.3(\mathrm{Cq}), 129.5(\mathrm{CH}), 129.4(\mathrm{CH}), 129.0(\mathrm{CH}), 128.7(\mathrm{CH}), 128.5(\mathrm{CH})$, $127.9(\mathrm{CH}), 127.7(\mathrm{CH}), 127.6(\mathrm{CH}), 126.3(\mathrm{CH}), 123.5(\mathrm{CH}), 122.5(\mathrm{CH}), 99.7\left(\mathrm{CH}_{2}\right), 87.5(\mathrm{CH})$, $48.5\left(\mathrm{CH}_{2}\right)$. (ESI)-HRMS calcd for $\mathrm{C}_{22} \mathrm{H}_{20} \mathrm{NO}_{2} \mathrm{~S}[\mathrm{M}+\mathrm{H}]^{+} 362.1209$, found 362.1206 . 
<smiles>C=C=CN(CC/C=C/c1ccccc1)S(=O)(=O)c1ccc(C)cc1</smiles>

The general procedure (GP-2) was followed using the correspondent enyne (140 mg, 0.41 $\mathrm{mmol})$. Purification by chromatography on silica gel yielded $3 \mathrm{~m}(55.8 \mathrm{mg}, 40 \%)$ as a viscous liquid. ${ }^{1} \mathrm{H}$ NMR (400 MHz, acetone) $\delta=7.76(\mathrm{~d}, J=8.4 \mathrm{~Hz}, 2 \mathrm{H}), 7.47-7.41(\mathrm{~m}, 2 \mathrm{H}), 7.40-7.34$ $(\mathrm{m}, 2 \mathrm{H}), 7.34-7.26(\mathrm{~m}, 2 \mathrm{H}), 7.25-7.16(\mathrm{~m}, 1 \mathrm{H}), 6.87(\mathrm{t}, J=6.3 \mathrm{~Hz}, 1 \mathrm{H}), 6.46(\mathrm{~d}, J=15.9 \mathrm{~Hz}$, $1 \mathrm{H}), 6.22(\mathrm{dt}, J=15.9,7.1 \mathrm{~Hz}, 1 \mathrm{H}), 5.42(\mathrm{~d}, J=6.3 \mathrm{~Hz}, 2 \mathrm{H}), 3.29(\mathrm{dd}, J=8.2,6.3 \mathrm{~Hz}, 2 \mathrm{H}), 2.57-$ $2.26(\mathrm{~m}, 5 \mathrm{H}) .{ }^{13} \mathrm{C}$ NMR (101 MHz, acetone) $\delta=200.6(\mathrm{Cq}), 144.0(\mathrm{Cq}), 137.5(\mathrm{Cq}), 135.7(\mathrm{Cq})$, $131.9(\mathrm{CH}), 129.9(\mathrm{CH}), 128.5(\mathrm{CH}), 127.2(\mathrm{CH}), 127.1(\mathrm{CH}), 126.3(\mathrm{CH}), 126.0(\mathrm{CH}), 99.8(\mathrm{CH})$, $87.3\left(\mathrm{CH}_{2}\right), 46.1\left(\mathrm{CH}_{2}\right), 31.6\left(\mathrm{CH}_{2}\right), 20.6\left(\mathrm{CH}_{3}\right)$. (ESI)-HRMS calcd for $\mathrm{C}_{20} \mathrm{H}_{21} \mathrm{NNaO}_{2} \mathrm{~S}[\mathrm{M}+\mathrm{Na}]^{+}$ 362.1185 , found 361.1192 . 


\section{Characterization of products}

\section{General procedure 3 (GP-3)}

To a vial charged with substrate 1 or $\mathbf{3}$ (1 equiv.) and complex $\mathbf{A}$ ( 1 mol\%), dry and degassed DMF (0.05 M) was added through a syringe. The solution was transferred in two NMR tubes capped with a rubber septum and it was then degassed through three freeze-pump cycles. The homogeneous solution was placed in an oil bath kept at $25{ }^{\circ} \mathrm{C}$ and irradiated with LED stripes for 6 hours (for 1,7-enallenes) or 24 hours (for 1,6-enallenes). Conversion was monitored by TLC and the mixture was then concentrated in vacuo. The residue was eventually purified by chromatography on silica gel.

(1R,5R,7R)-6-methylene-7-phenyl-3-tosyl-3-azabicyclo[3.2.0]heptane (2a)<smiles>[3H]N1CC2C(=C)[C@H](c3ccccc3)C2C1</smiles>

The general procedure (GP-3) was followed using 1a $(35 \mathrm{mg}, 0.1 \mathrm{mmol}$ ). Purification by chromatography on silica gel ( $n$-hexanes/EtOAc 90:10) yielded 2 a $(28.9 \mathrm{mg}, 82 \%)$ as a white solid. ${ }^{1} \mathrm{H}$ NMR $\left(400 \mathrm{MHz}, \mathrm{CDCl}_{3}\right) \delta=7.72(\mathrm{~d}, J=8.1 \mathrm{~Hz}, 2 \mathrm{H}), 7.39-7.27(\mathrm{~m}, 4 \mathrm{H}), 7.26-7.17$ $(\mathrm{m}, 3 \mathrm{H}), 5.05(\mathrm{t}, J=2.4 \mathrm{~Hz}, 1 \mathrm{H}), 4.88(\mathrm{t}, J=2.5 \mathrm{~Hz}, 1 \mathrm{H}), 3.95(\mathrm{br}, 1 \mathrm{H}), 3.69(\mathrm{dd}, J=9.7,4.5 \mathrm{~Hz}$, $2 \mathrm{H}), 3.42(\mathrm{br}, 1 \mathrm{H}), 2.89-2.65(\mathrm{~m}, 3 \mathrm{H}), 2.44(\mathrm{~s}, 3 \mathrm{H}) .{ }^{13} \mathrm{C} \mathrm{NMR}\left(101 \mathrm{MHz}, \mathrm{CDCl}_{3}\right) \delta=151.5(\mathrm{Cq})$, $143.7(\mathrm{Cq}), 142.3(\mathrm{Cq}), 131.9(\mathrm{Cq}), 129.6(\mathrm{CH}), 128.6(\mathrm{CH}), 128.1(\mathrm{CH}), 127.2(\mathrm{CH}), 126.6(\mathrm{CH})$, $110.1\left(\mathrm{CH}_{2}\right), 53.8\left(2 \mathrm{CH}_{2}\right), 53.0(\mathrm{CH}), 45.0(\mathrm{CH}), 43.9(\mathrm{CH}), 21.6\left(\mathrm{CH}_{3}\right)$. (ESI)-HRMS calcd for $\mathrm{C}_{20} \mathrm{H}_{21} \mathrm{NNaO}_{2} \mathrm{~S}[\mathrm{M}+\mathrm{Na}]^{+}$362.1185, found 362.1179 .

(1R,5R,6R)-6-(4-chlorophenyl)-7-methylene-3-tosyl-3-azabicyclo[3.2.0]heptane (2b)<smiles></smiles>

The general procedure (GP-3) was followed using $1 \mathbf{b}$ (75 $\mathrm{mg}, 0.2 \mathrm{mmol}$ ). Purification by chromatography on silica gel ( $n$-hexanes/EtOAc $90: 10)$ yielded $\mathbf{2 b}(40.1 \mathrm{mg}, 53 \%)$ as a white solid. ${ }^{1} \mathrm{H}$ NMR (300 MHz, $\left.\mathrm{CDCl}_{3}\right) \delta=7.72(\mathrm{~d}, J=8.0 \mathrm{~Hz}, 2 \mathrm{H}), 7.39-7.23(\mathrm{~m}, 4 \mathrm{H}), 7.16(\mathrm{~d}, J=8.2$ $\mathrm{Hz}, 2 \mathrm{H}), 5.06(\mathrm{~s}, 1 \mathrm{H}), 4.87(\mathrm{~s}, 1 \mathrm{H}), 3.93(\mathrm{br}, 1 \mathrm{H}), 3.68(\mathrm{dd}, J=9.7,4.6 \mathrm{~Hz}, 2 \mathrm{H}), 3.43(\mathrm{br}, 1 \mathrm{H}), 2.85$ - $2.62(\mathrm{~m}, 3 \mathrm{H}), 2.45(\mathrm{~s}, 3 \mathrm{H}) .{ }^{13} \mathrm{C}$ NMR (75 MHz, CDCl $) \delta=151.1(\mathrm{Cq}), 143.79(\mathrm{Cq}), 140.8(\mathrm{Cq})$, $132.3(\mathrm{Cq}), 131.8(\mathrm{Cq}), 129.6(\mathrm{CH}), 128.7(\mathrm{CH}), 128.6(\mathrm{CH}), 128.1(\mathrm{CH}), 110.3\left(\mathrm{CH}_{2}\right), 53.7\left(\mathrm{CH}_{2}\right)$, $53.7\left(\mathrm{CH}_{2}\right), 52.3(\mathrm{CH}), 45.0(\mathrm{CH}), 43.8(\mathrm{CH}), 21.6\left(\mathrm{CH}_{3}\right)$. (ESI)-HRMS calcd for $\mathrm{C}_{20} \mathrm{H}_{20} \mathrm{ClNNaO}_{2} \mathrm{~S}$ $[\mathrm{M}+\mathrm{Na}]^{+}$396.0795, found 396.0805 . 
<smiles></smiles>

The general procedure (GP-3) was followed using 1c $(80.9 \mathrm{mg}, 0.2 \mathrm{mmol})$. Purification by chromatography on silica gel ( $n$-hexanes/EtOAc $90: 10)$ yielded $2 \mathrm{c}(54.5 \mathrm{mg}, 67 \%)$ as a white solid. ${ }^{1} \mathrm{H}$ NMR $\left(400 \mathrm{MHz}, \mathrm{CDCl}_{3}\right) \delta=7.75(\mathrm{~d}, J=8.0 \mathrm{~Hz}, 2 \mathrm{H}), 7.58(\mathrm{~d}, J=8.0 \mathrm{~Hz}, 2 \mathrm{H}), 7.37$ (dd, $J$ $=8.2,4.0 \mathrm{~Hz}, 4 \mathrm{H}), 5.11(\mathrm{~s}, 1 \mathrm{H}), 4.90(\mathrm{~s}, 1 \mathrm{H}), 4.04(\mathrm{br}, 1 \mathrm{H}) 3.72(\mathrm{dd}, J=9.8,3.7 \mathrm{~Hz}, 2 \mathrm{H}), 3.47(\mathrm{br}$, $1 \mathrm{H}), 2.88-2.67(\mathrm{~m}, 3 \mathrm{H}), 2.46(\mathrm{~s}, 3 \mathrm{H}) .{ }^{13} \mathrm{C} \mathrm{NMR}\left(101 \mathrm{MHz}, \mathrm{CDCl}_{3}\right) \delta=150.6(\mathrm{Cq}), 146.3(\mathrm{q}, J=$ $1.5 \mathrm{~Hz}, \mathrm{Cq}), 143.9(\mathrm{Cq}), 131.6(\mathrm{Cq}), 129.7(\mathrm{CH}), 128.8(\mathrm{q}, J=32.2 \mathrm{~Hz}, \mathrm{Cq}), 128.1(\mathrm{CH}), 127.6(\mathrm{CH})$, $125.6(q, J=3.6 \mathrm{~Hz}, \mathrm{CH}), 124.2(\mathrm{q}, J=271.7 \mathrm{~Hz}, \mathrm{Cq}) 110.8\left(\mathrm{CH}_{2}\right), 53.75\left(\mathrm{CH}_{2}\right), 53.72\left(\mathrm{CH}_{2}\right), 53.6$ (CH), $45.0(\mathrm{CH}), 43.7(\mathrm{CH}), 21.6\left(\mathrm{CH}_{3}\right) .{ }^{19} \mathrm{~F} \mathrm{NMR}\left(564 \mathrm{MHz}, \mathrm{CDCl}_{3}\right) \delta=-62.28$. (ESI)-HRMS calcd for $\mathrm{C}_{21} \mathrm{H}_{20} \mathrm{~F}_{3} \mathrm{NNaO}_{2} \mathrm{~S}[\mathrm{M}+\mathrm{Na}]^{+} 430.1059$, found 429.1070 .

(1R,5R,6R)-6-(3-fluorophenyl)-7-methylene-3-tosyl-3-azabicyclo[3.2.0]heptane (2d)<smiles>[3H]N1CC2C(=C)[C@H](c3cccc(F)c3)C2C1</smiles>

The general procedure (GP-3) was followed using $1 \mathbf{d}(71.0 \mathrm{mg}, 02 \mathrm{mmol})$. Purification by chromatography on silica gel ( $n$-hexanes/EtOAc $90: 10)$ yielded $\mathbf{2 d}(39.0 \mathrm{mg}, 55 \%)$ as a white solid. ${ }^{1} \mathrm{H}$ NMR $\left(600 \mathrm{MHz}^{\mathrm{CDCl}}{ }_{3}\right) \delta=7.71(\mathrm{~d}, J=8.3 \mathrm{~Hz}, 2 \mathrm{H}), 7.33(\mathrm{~d}, J=8.0 \mathrm{~Hz}, 2 \mathrm{H}), 7.28-7.21$ $(\mathrm{m}, 1 \mathrm{H}), 6.97(\mathrm{~d}, J=7.9 \mathrm{~Hz}, 1 \mathrm{H}), 6.95-6.85(\mathrm{~m}, 2 \mathrm{H}), 5.07-5.03(\mathrm{~m}, 1 \mathrm{H}), 4.90-4.86(\mathrm{~m}, 1 \mathrm{H})$, $3.95-3.90(\mathrm{~m}, 1 \mathrm{H}), 3.70-3.60(\mathrm{~m}, 2 \mathrm{H}), 3.41(\mathrm{br}, 1 \mathrm{H}), 2.82-2.72(\mathrm{~m}, 2 \mathrm{H}), 2.69(\mathrm{dd}, J=10.0$, $5.9 \mathrm{~Hz}, 1 \mathrm{H}), 2.43(\mathrm{~s}, 3 \mathrm{H}) .{ }^{13} \mathrm{C}$ NMR $\left(151 \mathrm{MHz}^{\mathrm{C}} \mathrm{CDCl}_{3}\right) \delta=163.1(\mathrm{~d}, J=246.0 \mathrm{~Hz}, \mathrm{Cq}), 150.8(\mathrm{Cq})$, $144.9(\mathrm{~d}, J=6.7 \mathrm{~Hz}, \mathrm{Cq}), 143.9(\mathrm{Cq}), 131.99(\mathrm{Cq}), 130.14(\mathrm{~d}, J=8.2 \mathrm{~Hz}, \mathrm{CH}), 129.7(\mathrm{CH}), 128.2$ $(\mathrm{CH}), 123.0(\mathrm{~d}, J=2.8 \mathrm{~Hz}, \mathrm{CH}), 114.0(\mathrm{~d}, J=21.4 \mathrm{~Hz}, \mathrm{CH}), 113.5(\mathrm{~d}, J=21.0 \mathrm{~Hz}, \mathrm{CH}), 110.6\left(\mathrm{CH}_{2}\right)$, $53.80\left(\mathrm{CH}_{2}\right), 53.76\left(\mathrm{CH}_{2}\right), 52.6(\mathrm{CH}), 45.0(\mathrm{CH}), 43.8(\mathrm{CH}), 21.6\left(\mathrm{CH}_{3}\right) .{ }^{19} \mathrm{~F} \mathrm{NMR}\left(564 \mathrm{MHz}, \mathrm{CDCl}_{3}\right)$ $\delta=-112.87\left(q, J=8.7 \mathrm{~Hz}\right.$ ). (ESI)-HRMS calcd for $\mathrm{C}_{20} \mathrm{H}_{20} \mathrm{FNNaO}_{2} \mathrm{~S}[\mathrm{M}+\mathrm{Na}]^{+} 380.1091$, found 380.1079 . 
<smiles>[3H]N1CC2C(=C)[C@H](c3cccc(C#N)c3)C2C1</smiles>

The general procedure (GP-3) was followed using 1 e $(72.6 \mathrm{mg}, 0.20 \mathrm{mmol})$. Purification by chromatography on silica gel ( $n$-hexanes/EtOAc $65: 35)$ yielded 2 e $(49.0 \mathrm{mg}, 67 \%)$ as a white solid. ${ }^{1} \mathbf{H}$ NMR (400 MHz, $\left.\mathrm{CDCl}_{3}\right) \delta=7.73(\mathrm{~d}, J=8.3 \mathrm{~Hz}, 2 \mathrm{H}), 7.60-7.50(\mathrm{~m}, 2 \mathrm{H}), 7.51-7.29$ $(\mathrm{m}, 4 \mathrm{H}), 5.13(\mathrm{br}, 1 \mathrm{H}), 4.90(\mathrm{br}, 1 \mathrm{H}), 4.04-3.96(\mathrm{~m}, 1 \mathrm{H}), 3.71(\mathrm{dd}, J=9.8,4.2 \mathrm{~Hz}, 2 \mathrm{H}), 3.51-$ $3.42(\mathrm{~m}, 1 \mathrm{H}), 2.86-2.63(\mathrm{~m}, 3 \mathrm{H}), 2.46(\mathrm{~s}, 3 \mathrm{H}) .{ }^{13} \mathrm{C} \mathrm{NMR}\left(101 \mathrm{MHz}, \mathrm{CDCl}_{3}\right) \delta=150.1(\mathrm{Cq}), 143.9$ $(\mathrm{Cq}), 143.7(\mathrm{Cq}), 132.0(\mathrm{CH}), 131.6(\mathrm{Cq}), 130.7(\mathrm{CH}), 130.3(\mathrm{CH}), 129.7(\mathrm{CH}), 129.5(\mathrm{CH}), 128.1$ $(\mathrm{CH}), 118.9(\mathrm{Cq}), 112.7(\mathrm{Cq}), 111.1\left(\mathrm{CH}_{2}\right), 53.7\left(\mathrm{CH}_{2}\right), 53.6\left(\mathrm{CH}_{2}\right), 52.2(\mathrm{CH}), 45.0(\mathrm{CH}), 43.7(\mathrm{CH})$, $21.6\left(\mathrm{CH}_{3}\right)$. (ESI)-HRMS calcd for $\mathrm{C}_{21} \mathrm{H}_{20} \mathrm{~N}_{2} \mathrm{NaO}_{2} \mathrm{~S}[\mathrm{M}+\mathrm{Na}]^{+} 387.1138$, found 387.1146.

(1R,5R,7R)-6-methylene-7-(o-tolyl)-3-tosyl-3-azabicyclo[3.2.0]heptane (2f)<smiles>[3H]N1CC2C(=C)[C@H](c3ccccc3C)C2C1</smiles>

The general procedure (GP-3) was followed using $1 \mathrm{f}(66.3 \mathrm{mg}, 0.19 \mathrm{mmol})$. Purification by chromatography on silica gel ( $n$-hexanes/EtOAc $90: 10)$ yielded $\mathbf{2 f}(41.5 \mathrm{mg}, 63 \%)$ as a white solid. ${ }^{1} \mathrm{H}$ NMR $\left(400 \mathrm{MHz} \mathrm{CDCl}_{3}\right) \delta=7.73(\mathrm{~d}, J=8.3 \mathrm{~Hz}, 2 \mathrm{H}), 7.45-7.29(\mathrm{~m}, 3 \mathrm{H}), 7.23-7.07$ $(\mathrm{m}, 3 \mathrm{H}), 5.16(\mathrm{br}, 1 \mathrm{H}), 4.95(\mathrm{br}, 1 \mathrm{H}), 4.16-4.07(\mathrm{~m}, 1 \mathrm{H}), 3.73(\mathrm{t}, J=10.0 \mathrm{~Hz}, 2 \mathrm{H}), 3.43(\mathrm{br}, 1 \mathrm{H})$, $2.81-2.71(\mathrm{~m}, 2 \mathrm{H}), 2.71-2.63(\mathrm{~m}, 1 \mathrm{H}), 2.44(\mathrm{~s}, 3 \mathrm{H}), 2.27(\mathrm{~s}, 3 \mathrm{H}) .{ }^{13} \mathrm{C} \mathrm{NMR}\left(101 \mathrm{MHz}, \mathrm{CDCl}_{3}\right)$ $\delta=150.5(\mathrm{Cq}), 143.7(\mathrm{Cq}), 140.2(\mathrm{Cq}), 135.9(\mathrm{Cq}), 131.8(\mathrm{Cq}), 130.4(\mathrm{CH}), 129.6(\mathrm{CH}), 128.1$ $(\mathrm{CH}), 126.5(\mathrm{CH}), 126.1(\mathrm{CH}), 126.0(\mathrm{CH}), 111.0\left(\mathrm{CH}_{2}\right), 53.9\left(2 \mathrm{CH}_{2}\right), 50.0(\mathrm{CH}), 44.6(\mathrm{CH}), 43.5$ (CH), $21.6\left(\mathrm{CH}_{3}\right), 20.0\left(\mathrm{CH}_{3}\right)$. (ESI)-HRMS calcd for $\mathrm{C}_{21} \mathrm{H}_{23} \mathrm{NNaO}_{2} \mathrm{~S}[\mathrm{M}+\mathrm{Na}]^{+} 376.1342$, found 376.1351 . 
<smiles>C=C1C2CN([13CH3])C[C@H]2[C@H]1c1ccc(OC)cc1</smiles>

The general procedure (GP-3) was followed using $1 \mathrm{~g}(73.9 \mathrm{mg}, 0.20 \mathrm{mmol})$. Purification by chromatography on silica gel ( $n$-hexanes/EtOAc 90:10) yielded $\mathbf{2 g}(58.8 \mathrm{mg}, 80 \%, 6: 1$ mixture of diastereoisomers that are not separable by chromatography) as a white solid. Mayor diastereoisomer: ${ }^{1} \mathbf{H}$ NMR $\left(400 ~ M H z, \mathrm{CDCl}_{3}\right) \delta=7.72(\mathrm{~d}, J=8.3 \mathrm{~Hz}, 2 \mathrm{H}), 7.43-7.31(\mathrm{~m}, 2 \mathrm{H})$, $7.18-7.11(\mathrm{~m}, 2 \mathrm{H}), 6.90-6.77(\mathrm{~m}, 2 \mathrm{H}), 5.05-4.99(\mathrm{~m}, 1 \mathrm{H}), 4.88-4.82(\mathrm{~m}, 1 \mathrm{H}), 3.89(\mathrm{dq}, J=$ 5.7, $2.9 \mathrm{~Hz}, 1 \mathrm{H}), 3.78(\mathrm{~s}, 3 \mathrm{H}), 3.67(\mathrm{dd}, J=9.7,5.8 \mathrm{~Hz}, 2 \mathrm{H}), 3.46-3.36(\mathrm{~m}, 1 \mathrm{H}), 2.82-2.72(\mathrm{~m}$, $2 \mathrm{H}), 2.68(\mathrm{dd}, J=9.8,5.8 \mathrm{~Hz}, 1 \mathrm{H}), 2.44(\mathrm{~s}, 3 \mathrm{H}) .{ }^{13} \mathrm{C} \mathrm{NMR}\left(101 \mathrm{MHz}, \mathrm{CDCl}_{3}\right) \delta=158.3(\mathrm{Cq}), 152.1$ (Cq), $143.8(\mathrm{Cq}), 134.5(\mathrm{Cq}), 131.7(\mathrm{Cq}), 129.6(\mathrm{CH}), 128.3(\mathrm{CH}), 128.1(\mathrm{CH}), 114.0(\mathrm{CH}), 109.8$ $\left(\mathrm{CH}_{2}\right), 55.3\left(\mathrm{CH}_{3}\right), 53.8\left(2 \mathrm{CH}_{2}\right), 52.3(\mathrm{CH}), 44.9(\mathrm{CH}), 44.1(\mathrm{CH}), 21.6\left(\mathrm{CH}_{3}\right)$. (ESI)-HRMS calcd for $\mathrm{C}_{21} \mathrm{H}_{23} \mathrm{KNO}_{3} \mathrm{~S}[\mathrm{M}+\mathrm{K}]^{+}$408.1030, found 408.1033 .

(1R,5R,7R)-6-methylene-7-(pyridin-3-yl)-3-tosyl-3-azabicyclo[3.2.0]heptane. (2h)<smiles>[3H]N1CC2C(=C)[C@H](c3cccnc3)C2C1</smiles>

The general procedure (GP-3) was followed using $1 \mathrm{~h}(68.2 \mathrm{mg}, 0.20 \mathrm{mmol})$. Purification by chromatography on silica gel (DCM/MeOH 98:2) yielded $\mathbf{2 h}(40.7 \mathrm{mg}, 59 \%)$ as a blue/green solid. ${ }^{1} \mathrm{H}$ NMR $\left(400 \mathrm{MHz}^{\mathrm{CDCl}}{ }_{3}\right) \delta=7.71(\mathrm{~d}, J=8.3 \mathrm{~Hz}, 2 \mathrm{H}), 7.35(\mathrm{~d}, J=7.9 \mathrm{~Hz}, 2 \mathrm{H}), 5.09(\mathrm{~s}, 1 \mathrm{H})$, $4.87(\mathrm{~s}, 1 \mathrm{H}), 4.14(\mathrm{br}, 1 \mathrm{H}), 3.69(\mathrm{dd}, J=9.8,6.2 \mathrm{~Hz}, 2 \mathrm{H}), 3.52-3.41(\mathrm{~m}, 1 \mathrm{H}), 2.97-2.67(\mathrm{~m}$, $3 \mathrm{H}), 2.44(\mathrm{~s}, 3 \mathrm{H}) .{ }^{13} \mathrm{C}$ NMR (101 MHz, $\left.\mathrm{CDCl}_{3}\right) \delta=150.6(\mathrm{Cq}), 143.9(\mathrm{Cq}), 131.7(\mathrm{Cq}), 129.7(\mathrm{CH})$, $128.1(\mathrm{CH}), 110.9\left(\mathrm{CH}_{2}\right), 53.7\left(2 \mathrm{CH}_{2}\right), 45.2(\mathrm{CH}), 43.9(\mathrm{CH}), 21.6\left(\mathrm{CH}_{3}\right)$. Resonances of the 3pyridyl unit were barely observed even with prolonged relaxation times. (ESI)-HRMS calcd for $\mathrm{C}_{19} \mathrm{H}_{21} \mathrm{~N}_{2} \mathrm{O}_{2} \mathrm{~S}[\mathrm{M}+\mathrm{H}]^{+}$341.1324, found 341.1322.

(1R,5R,7R)-6-methylene-7-phenyl-3-oxabicyclo[3.2.0]heptane (2j)<smiles>C=C1C2COCC2[C@H]1c1ccccc1</smiles>

The general procedure (GP-3) was followed using $\mathbf{1 j}(55.0 \mathrm{mg}, 0.29 \mathrm{mmol})$. Purification by chromatography on silica gel ( $n$-hexanes/EtOAc 95:5) yielded $2 \mathbf{j}$ ( $27.0 \mathrm{mg}, 49 \%, 9: 1$ mixture of 
diastereoisomers that are not separable by chromatography) as a yellow oil. Major diastereoisomer: ${ }^{1} \mathbf{H}$ NMR (400 MHz, $\left.\mathbf{C D C l}_{3}\right) \delta=7.40-7.20(\mathrm{~m}, 5 \mathrm{H}), 5.04(\mathrm{dd}, J=2.9,1.9 \mathrm{~Hz}$, $1 \mathrm{H}), 4.86(\mathrm{t}, J=2.4 \mathrm{~Hz}, 1 \mathrm{H}), 4.14(\mathrm{dd}, J=9.1,7.5 \mathrm{~Hz}, 2 \mathrm{H}), 3.87-3.79(\mathrm{~m}, 1 \mathrm{H}), 3.66(\mathrm{dd}, J=8.9$, $6.2 \mathrm{~Hz}, 1 \mathrm{H}), 3.60(\mathrm{dd}, J=9.4,4.8 \mathrm{~Hz}, 2 \mathrm{H}), 3.01(\mathrm{dt}, J=7.5,5.0 \mathrm{~Hz}, 1 \mathrm{H}) .{ }^{13} \mathrm{C}$ NMR $(101 \mathrm{MHz}$, $\left.\mathrm{CDCl}_{3}\right) \delta=153.1(\mathrm{Cq}), 142.9(\mathrm{Cq}), 128.6(\mathrm{CH}), 127.3(\mathrm{CH}), 126.4(\mathrm{CH}), 108.7\left(\mathrm{CH}_{2}\right), 74.0\left(\mathrm{CH}_{2}\right)$, $73.6\left(\mathrm{CH}_{2}\right), 52.8(\mathrm{CH}), 46.8(\mathrm{CH}), 45.4(\mathrm{CH})$. Minor diastereoisomer: ${ }^{1} \mathrm{H}$ NMR (400 MHz, $\mathrm{CDCl}_{3}$ ) $\delta=7.43-7.20(\mathrm{~m}, 5 \mathrm{H}), 5.13(\mathrm{dd}, J=3.2,1.8 \mathrm{~Hz}, 1 \mathrm{H}), 4.97(\mathrm{t}, J=2.5 \mathrm{~Hz}, 1 \mathrm{H}), 4.36(\mathrm{dt}, J=9.3$, $3.0 \mathrm{~Hz}, 2 \mathrm{H}), 3.72-3.51(\mathrm{~m}, 2 \mathrm{H}), 3.41(\mathrm{dd}, J=9.9,6.1 \mathrm{~Hz}, 1 \mathrm{H}), 3.31-3.18(\mathrm{~m}, 2 \mathrm{H}) .{ }^{13} \mathrm{C} \mathrm{NMR}$ $\left(101 \mathrm{MHz}, \mathrm{CDCl}_{3}\right) \delta=153.5(\mathrm{Cq}), 138.2(\mathrm{Cq}), 128.8(\mathrm{CH}), 128.3(\mathrm{CH}), 126.6(\mathrm{CH}), 108.2\left(\mathrm{CH}_{2}\right)$, $73.7\left(\mathrm{CH}_{2}\right), 68.6\left(\mathrm{CH}_{2}\right), 49.2(\mathrm{CH}), 47.0(\mathrm{CH}), 42.4(\mathrm{CH})$. (ESI)-HRMS calcd for $\mathrm{C}_{13} \mathrm{H}_{14} \mathrm{NaO}[\mathrm{M}+\mathrm{Na}]^{+}$ 209.0937, found 209.0945.

(1R,5R,7S)-6-cyclohexylidene-7-phenyl-3-tosyl-3-azabicyclo[3.2.0]heptane (2k)<smiles>CN1CC2C(=C3CCCCC3)C(c3ccccc3)[C@H]2C1</smiles>

The general procedure (GP-3) was followed using $1 \mathbf{k}(81.5 \mathrm{mg}, 0.20 \mathrm{mmol})$. Purification by chromatography on silica gel ( $n$-hexanes/EtOAc 90:10) yielded $\mathbf{2 k}(54.2 \mathrm{mg}, 66 \%)$ as a white solid. ${ }^{1} \mathrm{H}$ NMR (400 MHz, $\left.\mathrm{CDCl}_{3}\right) \delta=7.74(\mathrm{~d}, J=8.2 \mathrm{~Hz}, 2 \mathrm{H}), 7.51-7.09(\mathrm{~m}, 7 \mathrm{H}), 3.88(\mathrm{br}, 1 \mathrm{H})$, $3.70(\mathrm{~d}, J=9.8 \mathrm{~Hz}, 1 \mathrm{H}), 3.63(\mathrm{~d}, J=9.3 \mathrm{~Hz}, 1 \mathrm{H}), 3.54-3.41(\mathrm{~m}, 1 \mathrm{H}), 2.69$ (ddd, $J=15.5,9.5,6.4$ $\mathrm{Hz}, 2 \mathrm{H}), 2.62-2.53(\mathrm{~m}, 1 \mathrm{H}), 2.46(\mathrm{~s}, 3 \mathrm{H}), 2.13-1.96(\mathrm{~m}, 2 \mathrm{H}), 1.81-1.17(\mathrm{~m}, 8 \mathrm{H}) .{ }^{13} \mathrm{C}$ NMR $\left(101 \mathrm{MHz}, \mathrm{CDCl}_{3}\right) \delta=144.1(\mathrm{Cq}), 143.6(\mathrm{Cq}), 136.3(\mathrm{Cq}), 131.8(\mathrm{Cq}), 129.6(\mathrm{CH}), 129.2(\mathrm{Cq})$, $128.5(\mathrm{CH}), 128.1(\mathrm{CH}), 127.1(\mathrm{CH}), 126.2(\mathrm{CH}), 53.9\left(\mathrm{CH}_{2}\right), 53.7\left(\mathrm{CH}_{2}\right), 51.5(\mathrm{CH}), 44.0(\mathrm{CH}), 42.4$ (CH), $29.3\left(\mathrm{CH}_{2}\right), 29.3\left(\mathrm{CH}_{2}\right), 27.7\left(\mathrm{CH}_{2}\right), 26.8\left(\mathrm{CH}_{2}\right), 26.3\left(\mathrm{CH}_{2}\right), 21.6\left(\mathrm{CH}_{3}\right)$. (ESI)-HRMS calcd for $\mathrm{C}_{25} \mathrm{H}_{29} \mathrm{NNaO}_{2} \mathrm{~S}[\mathrm{M}+\mathrm{Na}]^{+} 430.1811$, found 430.1804 .

(1R,5R,6S)-6-phenyl-7-propylidene-3-tosyl-3-azabicyclo[3.2.0]heptane (2i)<smiles>[3H]N1CC2C(=CCC)C(C1)C2c1ccccc1</smiles>

The general procedure (GP-3) was followed using 1i (36mg, $0.10 \mathrm{mmol}$ ). Purification by chromatography on silica gel ( $n$-hexanes/EtOAc 90:10) yielded $\mathbf{2 i}$ ( $25.6 \mathrm{mg}, 71 \%, 5: 1$ mixture of $E / Z$ isomers that are not separable by chromatography) as a white solid. Mayor isomer: ${ }^{1} \mathbf{H}$ NMR (400 MHz, acetone) $\delta=7.71(\mathrm{~d}, J=8.3 \mathrm{~Hz}, 2 \mathrm{H}), 7.46(\mathrm{~d}, J=8.0 \mathrm{~Hz}, 2 \mathrm{H}), 7.33-7.25(\mathrm{~m}$, $4 \mathrm{H}), 7.24-7.13(\mathrm{~m}, 1 \mathrm{H}), 5.38(\mathrm{tt}, J=7.3,2.3 \mathrm{~Hz}, 1 \mathrm{H}), 3.85(\mathrm{br}, 1 \mathrm{H}), 3.68-3.51(\mathrm{~m}, 2 \mathrm{H}), 3.49$ (br, 1H), $2.88-2.62(\mathrm{~m}, 3 \mathrm{H}), 2.44(\mathrm{~s}, 3 \mathrm{H}), 1.74-1.58(\mathrm{~m}, 2 \mathrm{H}), 0.71(\mathrm{t}, J=7.5 \mathrm{~Hz}, 3 \mathrm{H}) .{ }^{13} \mathrm{C}$ NMR (101 MHz, acetone) $\delta=143.7(\mathrm{Cq}), 143.6(\mathrm{Cq}), 141.3(\mathrm{Cq}), 132.1(\mathrm{Cq}), 129.6(\mathrm{CH}), 128.5(\mathrm{CH})$, $128.1(\mathrm{CH}), 127.1(\mathrm{CH}), 127.0(\mathrm{CH}), 126.2(\mathrm{CH}), 54.4\left(\mathrm{CH}_{2}\right), 53.8\left(\mathrm{CH}_{2}\right), 51.9(\mathrm{CH}), 44.4(\mathrm{CH}), 44.1$ 
(CH), $21.0\left(\mathrm{CH}_{2}\right), 20.6\left(\mathrm{CH}_{3}\right), 12.9\left(\mathrm{CH}_{3}\right)$. (ESI)-HRMS calcd for $\mathrm{C}_{22} \mathrm{H}_{25} \mathrm{KNO}_{2} \mathrm{~S}[\mathrm{M}+\mathrm{K}]^{+}$406.1238, found 406.1234.

(1R,5R,7S)-6-benzylidene-7-phenyl-3-tosyl-3-azabicyclo[3.2.0]heptane (2I)<smiles>[AsH3-]N1CC2C(=Cc3ccccc3)C(c3ccccc3)C2C1</smiles>

The general procedure (GP-3) was followed using 11 (75mg, $0.18 \mathrm{mmol})$. Purification by chromatography on silica gel (gradient from $n$-hexanes/EtOAc $96: 4$ to $90: 10$ ) yielded $2 \mathbf{2}$ (29.3 $\mathrm{mg}, 39 \%, 1: 1$ mixture of $E / Z$ isomers, which were separated by chromatography) as a white solid. Isomer A: ${ }^{1} \mathrm{H}$ NMR $\left(400 \mathrm{MHz}, \mathrm{CDCl}_{3}\right) \delta=7.74(\mathrm{~d}, J=8.1 \mathrm{~Hz}, 2 \mathrm{H}), 7.42-7.15(\mathrm{~m}, 12 \mathrm{H})$, $6.07(\mathrm{t}, J=2.3 \mathrm{~Hz}, 1 \mathrm{H}), 4.12-4.05(\mathrm{~m}, 1 \mathrm{H}), 3.92-3.80(\mathrm{~m}, 2 \mathrm{H}), 3.73(\mathrm{~d}, J=10.0 \mathrm{~Hz}, 1 \mathrm{H}), 2.95$ (td, $J=7.7,2.1 \mathrm{~Hz}, 2 \mathrm{H}), 2.77(\mathrm{dd}, J=9.9,5.7 \mathrm{~Hz}, 1 \mathrm{H}), 2.45(\mathrm{~s}, 3 \mathrm{H}) .{ }^{13} \mathrm{C} \mathrm{NMR}\left(101 \mathrm{MHz}, \mathrm{CDCl}_{3}\right) \delta$ $=144.2(\mathrm{Cq}), 143.8(\mathrm{Cq}), 142.5(\mathrm{Cq}), 136.4(\mathrm{Cq}), 131.8(\mathrm{Cq}), 129.7(\mathrm{CH}), 128.7(\mathrm{CH}), 128.6(\mathrm{CH})$, $128.2(\mathrm{CH}), 127.7(\mathrm{CH}), 127.5(\mathrm{CH}), 126.8(2 \mathrm{CH}), 125.2(\mathrm{CH}), 54.0\left(\mathrm{CH}_{2}\right), 53.7\left(\mathrm{CH}_{2}\right), 52.2(\mathrm{CH})$, $45.4(\mathrm{CH}), 45.2(\mathrm{CH}), 21.6\left(\mathrm{CH}_{3}\right)$. (ESI)-MS calcd for $\mathrm{C}_{26} \mathrm{H}_{25} \mathrm{KNO}_{2} \mathrm{~S}[\mathrm{M}+\mathrm{K}]{ }^{+} 454.12$ found 454.35. Isomer B: ${ }^{1} \mathrm{H}$ NMR $\left(400 \mathrm{MHz}, \mathrm{CDCl}_{3}\right) \delta=7.72(\mathrm{~d}, J=8.3 \mathrm{~Hz}, 2 \mathrm{H}), 7.33-6.96(\mathrm{~m}, 12 \mathrm{H}), 6.36(\mathrm{t}, J$ $=2.3 \mathrm{~Hz}, 1 \mathrm{H}), 4.24-4.21(\mathrm{~m}, 1 \mathrm{H}), 3.83-3.72(\mathrm{~m}, 2 \mathrm{H}), 3.65-3.54(\mathrm{~m}, 1 \mathrm{H}), 2.87-2.73(\mathrm{~m}, 3 \mathrm{H})$, $2.40(\mathrm{~s}, 3 \mathrm{H}) .{ }^{13} \mathrm{C}$ NMR (101 MHz, $\left.\mathrm{CDCl}_{3}\right) \delta=143.80(\mathrm{Cq}), 142.8(\mathrm{Cq}), 141.5(\mathrm{Cq}), 135.9(\mathrm{Cq})$, $132.1(\mathrm{Cq}), 129.7(\mathrm{CH}), 128.9(\mathrm{CH}), 128.3(\mathrm{CH}), 128.2(\mathrm{CH}), 128.2(\mathrm{CH}), 127.1(\mathrm{CH}), 126.8(\mathrm{CH})$, $126.7(\mathrm{CH}), 126.3(\mathrm{CH}), 54.6\left(\mathrm{CH}_{2}\right), 54.1\left(\mathrm{CH}_{2}\right), 53.9(\mathrm{CH}), 46.1(\mathrm{CH}), 45.7(\mathrm{CH}), 21.7\left(\mathrm{CH}_{3}\right)$. (ESI)HRMS calcd for $\mathrm{C}_{26} \mathrm{H}_{25} \mathrm{KNO}_{2} \mathrm{~S}[\mathrm{M}+\mathrm{K}]^{+} 454.1238$, found 454.1239 .

$(1 R, 7 R, 8 S)-7,8-d i p h e n y l-3-t o s y l-3-a z a b i c y c l o[4.2 .0]$ oct-5-ene (2'-I)<smiles>CN1CC=C2C(C1)[C@H](c1ccccc1)C2c1ccccc1</smiles>

The general procedure (GP-3) was followed using $\mathbf{1 l}$ (75mg, $0.18 \mathrm{mmol}$ ). Purification by chromatography on silica gel (gradient from $n$-hexanes/EtOAc $96: 4$ to 90:10) yielded 2'-I (26.2 $\mathrm{mg}, 35 \%)$ as a white solid. ${ }^{1} \mathbf{H}$ NMR $\left(600 \mathrm{MHz}, \mathrm{CDCl}_{3}\right) \delta=7.71(\mathrm{~d}, J=8.3 \mathrm{~Hz}, 2 \mathrm{H}), 7.36-7.28$ $(\mathrm{m}, 6 \mathrm{H}), 7.25-7.16(\mathrm{~m}, 6 \mathrm{H}), 5.29(\mathrm{~s}, 1 \mathrm{H}), 4.44-4.27(\mathrm{~m}, 2 \mathrm{H}), 4.23-4.18(\mathrm{~m}, 1 \mathrm{H}), 3.35-3.26$ $(\mathrm{m}, 2 \mathrm{H}), 3.24(\mathrm{t}, J=8.4 \mathrm{~Hz}, 1 \mathrm{H}), 2.50-2.36(\mathrm{~m}, 4 \mathrm{H}) .{ }^{13} \mathrm{C} \mathrm{NMR}\left(151 \mathrm{MHz}, \mathrm{CDCl}_{3}\right) \delta=143.6(\mathrm{Cq})$, $141.8(\mathrm{Cq}), 141.0(\mathrm{Cq}), 139.5(\mathrm{Cq}), 134.4(\mathrm{Cq}), 129.8(\mathrm{CH}), 128.7(\mathrm{CH}), 128.6(\mathrm{CH}), 127.5(\mathrm{CH})$, $127.1(\mathrm{CH}), 126.9(\mathrm{CH}), 126.6(\mathrm{CH}), 109.8(\mathrm{CH}), 57.5(\mathrm{CH}), 51.0(\mathrm{CH}), 47.7\left(\mathrm{CH}_{2}\right), 45.0\left(\mathrm{CH}_{2}\right), 44.9$ (CH), $21.6\left(\mathrm{CH}_{3}\right)$. (ESI)-HRMS calcd for $\mathrm{C}_{26} \mathrm{H}_{25} \mathrm{KNO}_{2} \mathrm{~S}[\mathrm{M}+\mathrm{K}]^{+} 438.1498$, found 438.1510 . 


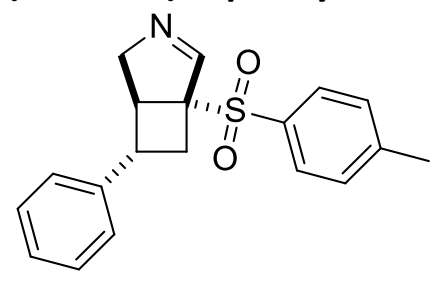

The general procedure (GP-3) was followed using 3a $(32.7 \mathrm{mg}, 0.10 \mathrm{mmol})$. Purification by chromatography on silica gel ( $n$-hexanes/EtOAc/DCM 50:40:10) yielded 4a (19.3mg, 59\%) as a pale yellow solid. ${ }^{1} \mathbf{H}$ NMR $\left(400 \mathbf{~ M H z}, \mathbf{C D C l}_{3}\right) \delta=7.74(\mathrm{~d}, J=7.5 \mathrm{~Hz}, 2 \mathrm{H}), 7.60(\mathrm{~s}, 1 \mathrm{H}), 7.43-$ $7.20(\mathrm{~m}, 7 \mathrm{H}), 3.97(\mathrm{~d}, J=17.0 \mathrm{~Hz}, 1 \mathrm{H}), 3.53$ (ddd, $J=16.9,6.4,2.9 \mathrm{~Hz}, 1 \mathrm{H}), 3.32(\mathrm{t}, J=6.5 \mathrm{~Hz}$, $1 \mathrm{H}), 3.20(\mathrm{dd}, J=12.2,8.9 \mathrm{~Hz}, 1 \mathrm{H}), 2.93(\mathrm{q}, J=8.2 \mathrm{~Hz}, 1 \mathrm{H}), 2.73-2.62(\mathrm{~m}, 1 \mathrm{H}), 2.45(\mathrm{~s}, 3 \mathrm{H}) .{ }^{13} \mathrm{C}$ NMR (101 MHz, CDCl $) \delta=161.4(\mathrm{CH}), 145.5(\mathrm{Cq}), 142.2(\mathrm{Cq}), 133.5(\mathrm{Cq}), 130.1(\mathrm{CH}), 128.9$ $(\mathrm{CH}), 128.8(\mathrm{CH}), 127.2(\mathrm{CH}), 127.0(\mathrm{CH}), 76.0(\mathrm{Cq}), 68.0\left(\mathrm{CH}_{2}\right), 47.2(\mathrm{CH}), 40.4(\mathrm{CH}), 33.5\left(\mathrm{CH}_{2}\right)$, $21.7\left(\mathrm{CH}_{3}\right)$. (ESI)-HRMS calcd for $\mathrm{C}_{19} \mathrm{H}_{20} \mathrm{NO}_{2} \mathrm{~S}[\mathrm{M}+\mathrm{H}]^{+} 326.1209$, found 326.1220 .

(1R,5S,6S)-6-(o-tolyl)-1-tosyl-3-azabicyclo[3.2.0]hept-2-ene (4b)<smiles>Cc1ccc(S(=O)(=O)[C@]23C=NCC2[C@@H](c2ccccc2C)C3)cc1</smiles>

The general procedure (GP-3) was followed using $3 \mathbf{b}(64.0 \mathrm{mg}, 0.20 \mathrm{mmol})$. Purification by chromatography on silica gel ( $n$-hexanes/EtOAc/DCM 50:40:10) yielded $\mathbf{4 b}$ (37.3 mg, 58\%) as a white solid. ${ }^{1} \mathrm{H}$ NMR $\left(400 \mathrm{MHz}, \mathrm{CDCl}_{3}\right) \delta=7.74(\mathrm{~d}, J=8.0 \mathrm{~Hz}, 2 \mathrm{H}), 7.64(\mathrm{~s}, 1 \mathrm{H}), 7.49(\mathrm{~d}, J=7.8$ $\mathrm{Hz}, 1 \mathrm{H}), 7.35(\mathrm{~d}, J=8.0 \mathrm{~Hz}, 2 \mathrm{H}), 7.31-7.24(\mathrm{~m}, 1 \mathrm{H}), 7.22-7.08(\mathrm{~m}, 2 \mathrm{H}), 3.98(\mathrm{~d}, J=17.0 \mathrm{~Hz}$, $1 \mathrm{H}), 3.58(\mathrm{ddd}, J=17.0,6.4,2.9 \mathrm{~Hz}, 1 \mathrm{H}), 3.39(\mathrm{t}, J=6.3 \mathrm{~Hz}, 1 \mathrm{H}), 3.24-3.15(\mathrm{~m}, 2 \mathrm{H}), 2.74-2.61$ (m, 1H), 2.45 (s, 3H), 2.21 (s, 3H). ${ }^{13} \mathrm{C}$ NMR (101 MHz, CDCl $\left.{ }_{3}\right) \delta=160.6(\mathrm{CH}), 145.5(\mathrm{Cq}), 139.9$ (Cq), $135.3(\mathrm{Cq}), 133.4(\mathrm{Cq}), 130.3(\mathrm{CH}), 130.1(\mathrm{CH}), 128.9(\mathrm{CH}), 126.9(\mathrm{CH}), 126.8(\mathrm{CH}), 126.1$ (CH), $76.0(\mathrm{Cq}), 68.2\left(\mathrm{CH}_{2}\right), 45.9(\mathrm{CH}), 36.3(\mathrm{CH}), 33.0\left(\mathrm{CH}_{2}\right), 21.7\left(\mathrm{CH}_{3}\right), 20.1\left(\mathrm{CH}_{3}\right)$. (ESI)-HRMS calcd for $\mathrm{C}_{20} \mathrm{H}_{22} \mathrm{NO}_{2} \mathrm{~S}[\mathrm{M}+\mathrm{H}]^{+} 340.1366$, found 340.1372 .

1R,5S,6S)-6-(4-methoxyphenyl)-1-tosyl-3-azabicyclo[3.2.0]hept-2-ene (4c)<smiles>COc1ccc([C@@H]2C[C@H]3CN=C[C@]32S(=O)(=O)c2ccc(C)cc2)cc1</smiles>

The general procedure (GP-3) was followed using 3c $(36.6 \mathrm{mg}, 0.10 \mathrm{mmol})$. Purification by chromatography on silica gel ( $n$-hexanes/EtOAc/DCM 50:40:10) yielded 4c (18.3 mg, 53\%) as a yellow solid. ${ }^{1} \mathbf{H}$ NMR $\left(300 \mathbf{M H z} \mathrm{CDCl}_{3}\right) \delta=7.74(\mathrm{~d}, J=8.3 \mathrm{~Hz}, 2 \mathrm{H}), 7.60(\mathrm{~s}, 1 \mathrm{H}), 7.35(\mathrm{~d}, J=$ $8.0 \mathrm{~Hz}, 2 \mathrm{H}), 7.29-7.17(\mathrm{~m}, 2 \mathrm{H}), 6.88(\mathrm{~d}, J=8.6 \mathrm{~Hz}, 2 \mathrm{H}), 3.96(\mathrm{~d}, J=16.7 \mathrm{~Hz}, 1 \mathrm{H}), 3.80(\mathrm{~s}, 3 \mathrm{H})$, 
3.53 (ddd, $J=16.8,6.3,2.5 \mathrm{~Hz}, 1 \mathrm{H}$ ), 3.27 (t, $J=6.4 \mathrm{~Hz}, 1 \mathrm{H}$ ), 3.15 (dd, $J=12.1,8.7 \mathrm{~Hz}, 1 \mathrm{H}$ ), 2.88 $(\mathrm{td}, J=8.6,6.6 \mathrm{~Hz}, 1 \mathrm{H}), 2.66(\mathrm{dd}, J=12.1,8.6 \mathrm{~Hz}, 1 \mathrm{H}), 2.45(\mathrm{~s}, 3 \mathrm{H}) .{ }^{13} \mathrm{C} \mathrm{NMR}\left(75 \mathrm{MHz}, \mathrm{CDCl}_{3}\right) \delta$ $=158.8(\mathrm{Cq}, \mathrm{CH}), 145.5(\mathrm{Cq}), 134.3(\mathrm{Cq}), 133.5(\mathrm{Cq}), 130.0(\mathrm{CH}), 129.2(\mathrm{Cq}), 128.9(\mathrm{CH}), 128.1$ (CH), $114.1(\mathrm{CH}), 67.9(\mathrm{Cq}), 55.3(\mathrm{CH} 3), 39.8(\mathrm{CH}), 33.8(\mathrm{CH}), 29.7(\mathrm{CH}), 21.7(\mathrm{CH} 3)$. (ESI)-HRMS calcd for $\mathrm{C}_{20} \mathrm{H}_{21} \mathrm{NKO}_{3} \mathrm{~S}[\mathrm{M}+\mathrm{K}]^{+} 394.0874$, found 394.0867.

(1R,5S,6S)-6-(4-chlorophenyl)-1-tosyl-3-azabicyclo[3.2.0]hept-2-ene (4d)<smiles>Cc1ccc(S(=O)(=O)C23CCN(C[C@H]2c2ccc(Cl)cc2)C3)cc1</smiles>

The general procedure (GP-3) was followed using $\mathbf{3 d}(71.0 \mathrm{mg}, 0.20 \mathrm{mmol})$. Purification by chromatography on silica gel ( $n$-hexanes/EtOAc/DCM 50:40:10) yielded $\mathbf{4 d}(27.0 \mathrm{mg}, 38 \%)$ as a white solid. ${ }^{1} \mathbf{H}$ NMR $\left(400 \mathrm{MHz}, \mathrm{CDCl}_{3}\right) \delta=7.73(\mathrm{~d}, J=8.0 \mathrm{~Hz}, 2 \mathrm{H}), 7.58(\mathrm{~s}, 1 \mathrm{H}), 7.46-7.06$ $(\mathrm{m}, 6 \mathrm{H}), 3.96(\mathrm{~d}, J=17.1 \mathrm{~Hz}, 1 \mathrm{H}), 3.60-3.48(\mathrm{~m}, 1 \mathrm{H}), 3.27(\mathrm{t}, J=6.5 \mathrm{~Hz}, 1 \mathrm{H}), 3.14(\mathrm{dd}, J=12.3$, $8.8 \mathrm{~Hz}, 1 \mathrm{H}), 2.91(\mathrm{q}, J=8.5 \mathrm{~Hz}, 1 \mathrm{H}), 2.68(\mathrm{dd}, J=12.3,8.6 \mathrm{~Hz}, 1 \mathrm{H}), 2.45(\mathrm{~s}, 3 \mathrm{H}) .{ }^{13} \mathrm{C}$ NMR (101 $\left.\left.\mathrm{MHz}_{\mathrm{CDCl}}\right)\right) \delta=161.3(\mathrm{CH}), 145.6(\mathrm{Cq}), 140.7(\mathrm{Cq}), 134.0(\mathrm{Cq}), 133.0(\mathrm{Cq}), 130.1(\mathrm{CH}), 128.9$ $(\mathrm{CH}), 128.9(\mathrm{CH}), 128.4(\mathrm{CH}), 76.0(\mathrm{Cq}), 67.9\left(\mathrm{CH}_{2}\right), 47.1(\mathrm{CH}), 39.8(\mathrm{CH}), 33.5\left(\mathrm{CH}_{2}\right), 20.7\left(\mathrm{CH}_{3}\right)$. (ESI)-HRMS calcd for $\mathrm{C}_{19} \mathrm{H}_{19} \mathrm{CINO}_{2} \mathrm{~S}[\mathrm{M}+\mathrm{H}]^{+} 360.0820$, found 360.0811 .

3-((1R,5S,6S)-1-tosyl-3-azabicyclo[3.2.0]hept-2-en-6-yl)benzonitrile (4e)<smiles>Cc1ccc(S(=O)(=O)C23C=NCC2[C@H](c2cccc(C#N)c2)C3)cc1</smiles>

The general procedure (GP-3) was followed using $3 e(45.0 \mathrm{mg}, 0.13 \mathrm{mmol})$. Purification by chromatography on silica gel ( $n$-hexanes/EtOAc/DCM 50:40:10) yielded 4 e $(14.0 \mathrm{mg}, 31 \%)$ as a yellow solid. ${ }^{1} \mathrm{H}$ NMR $\left(400 \mathrm{MHz}, \mathrm{CDCl}_{3}\right) \delta=7.74(\mathrm{~d}, J=8.3 \mathrm{~Hz}, 2 \mathrm{H}), 7.66-7.58(\mathrm{~m}, 2 \mathrm{H}), 7.56$ (dt, $J=7.7,1.4 \mathrm{~Hz}, 1 \mathrm{H}), 7.52-7.43(\mathrm{~m}, 2 \mathrm{H}), 7.37(\mathrm{~d}, J=8.0 \mathrm{~Hz}, 2 \mathrm{H}), 3.99(\mathrm{~d}, J=17.1 \mathrm{~Hz}, 1 \mathrm{H})$, 3.61 (ddd, $J=17.0,6.4,2.9 \mathrm{~Hz}, 1 \mathrm{H}$ ), $3.30(\mathrm{t}, J=6.4 \mathrm{~Hz}, 1 \mathrm{H}$ ), $3.14(\mathrm{dd}, J=12.3,8.7 \mathrm{~Hz}, 1 \mathrm{H}), 2.98$ $(\mathrm{td}, J=8.6,6.5 \mathrm{~Hz}, 1 \mathrm{H}), 2.73(\mathrm{dd}, J=12.5,8.8 \mathrm{~Hz}, 1 \mathrm{H}), 2.46(\mathrm{~s}, 3 \mathrm{H}) \cdot{ }^{13} \mathrm{C} \mathbf{N M R}\left(101 \mathrm{MHz}, \mathrm{CDCl}_{3}\right)$ $\delta=161.1(\mathrm{CH}), 145.9(\mathrm{Cq}), 143.6(\mathrm{Cq}), 133.1(\mathrm{Cq}), 131.5(\mathrm{CH}), 131.0(\mathrm{CH}), 130.6(\mathrm{CH}), 130.2$ $(\mathrm{CH}), 129.8(\mathrm{CH}), 128.9(\mathrm{CH}), 118.6(\mathrm{Cq}), 112.8(\mathrm{Cq}), 76.0(\mathrm{Cq}), 68.0\left(\mathrm{CH}_{2}\right), 46.8(\mathrm{CH}), 39.8(\mathrm{CH})$, $33.3\left(\mathrm{CH}_{2}\right), 21.8\left(\mathrm{CH}_{3}\right)$. (ESI)-HRMS calcd for $\mathrm{C}_{20} \mathrm{H}_{19} \mathrm{~N}_{2} \mathrm{O}_{2} \mathrm{~S}[\mathrm{M}+\mathrm{H}]^{+} 351.1162$, found 350.1149. 
(1R,5S,6S)-1-(methylsulfonyl)-6-phenyl-3-azabicyclo[3.2.0]hept-2-ene (4f)<smiles>CS(=O)(=O)[C@]12CN=C[C@H]1C[C@H]2c1ccccc1</smiles>

The general procedure (GP-3) was followed using $3 \mathrm{f}(26.4 \mathrm{mg}, 0.1 \mathrm{mmol})$. Purification by preparative TLC on silica gel ( $n$-hexanes/EtOAc/DCM 50:40:10) yielded $\mathbf{4 f}$ ( $14.5 \mathrm{mg}, 55 \%$ ) as a pale brown solid. ${ }^{1} \mathbf{H}$ NMR $\left(400 \mathrm{MHz}, \mathrm{CDCl}_{3}\right) \delta=7.80(\mathrm{t}, \mathrm{J}=2.6 \mathrm{~Hz}, 1 \mathrm{H}), 7.40-7.14(\mathrm{~m}, 5 \mathrm{H})$, $4.29-4.09(\mathrm{~m}, 2 \mathrm{H}), 3.41(\mathrm{t}, J=6.2 \mathrm{~Hz}, 1 \mathrm{H}), 3.18-3.00(\mathrm{~m}, 2 \mathrm{H}), 2.80-2.69(\mathrm{~m}, 4 \mathrm{H}) .{ }^{13} \mathrm{C}$ NMR $\left(101 \mathrm{MHz}, \mathrm{CDCl}_{3}\right) \delta=161.4(\mathrm{CH}), 141.0(\mathrm{Cq}), 129.0(\mathrm{CH}), 127.5(\mathrm{CH}), 127.1(\mathrm{CH}), 75.2(\mathrm{Cq}), 68.4$ $\left(\mathrm{CH}_{2}\right), 46.7(\mathrm{CH}), 41.0(\mathrm{CH}), 37.3\left(\mathrm{CH}_{3}\right), 32.7\left(\mathrm{CH}_{2}\right)$. (ESI)-HRMS calcd for $\mathrm{C}_{13} \mathrm{H}_{16} \mathrm{NO}_{2} \mathrm{~S}[\mathrm{M}+\mathrm{H}]^{+}$ 250.0896 , found 249.0905 .

(1R,5S,6S)-1-(methylsulfonyl)-6-(o-tolyl)-3-azabicyclo[3.2.0]hept-2-ene (4g)<smiles>Cc1ccccc1[C@H]1CC=NC[C@@H](S(C)(=O)=O)C1</smiles>

The general procedure (GP-3) was followed using $\mathbf{3 g}(43.7 \mathrm{mg}, 1.7 \mathrm{mmol})$. Purification by preparative TLC on silica gel ( $n$-hexanes/EtOAC/DCM 50:40:10) yielded $4 \mathrm{~g}(21.3 \mathrm{mg}, 49 \%)$ as a viscous yellow oil. ${ }^{1} \mathrm{H}$ NMR $\left(400 \mathrm{MHz}, \mathrm{CDCl}_{3}\right) \delta=7.83(\mathrm{~s}, 1 \mathrm{H}), 7.50(\mathrm{dd}, J=7.9,1.3 \mathrm{~Hz}, 1 \mathrm{H}), 7.34$ $-7.23(\mathrm{~m}, 1 \mathrm{H}), 7.23-7.09(\mathrm{~m}, 2 \mathrm{H}), 4.30-4.14(\mathrm{~m}, 2 \mathrm{H}), 3.53-3.44(\mathrm{~m}, 1 \mathrm{H}), 3.30(\mathrm{td}, J=8.8$, $6.8 \mathrm{~Hz}, 1 \mathrm{H}), 3.08(\mathrm{dd}, J=12.3,9.0 \mathrm{~Hz}, 1 \mathrm{H}), 2.81-2.71(\mathrm{~m}, 4 \mathrm{H}), 2.25(\mathrm{~s}, 3 \mathrm{H}) .{ }^{13} \mathrm{C} \mathrm{NMR}(101 \mathrm{MHz}$, $\left.\mathrm{CDCl}_{3}\right) \delta=161.4(\mathrm{CH}), 139.6(\mathrm{Cq}), 135.2(\mathrm{Cq}), 130.3(\mathrm{CH}), 127.1(\mathrm{CH}), 126.8(\mathrm{CH}), 126.0(\mathrm{CH})$, $75.1(\mathrm{Cq}), 68.5\left(\mathrm{CH}_{2}\right), 45.2(\mathrm{CH}), 37.2\left(\mathrm{CH}_{3}\right), 36.9(\mathrm{CH}), 32.2\left(\mathrm{CH}_{2}\right), 20.15\left(\mathrm{CH}_{3}\right)$. (ESI)-HRMS calcd for $\mathrm{C}_{14} \mathrm{H}_{17} \mathrm{NNaO}_{2} \mathrm{~S}[\mathrm{M}+\mathrm{Na}]^{+} 286.0872$, found 286.0861 .

(1R,5S,6S)-6-phenyl-1-(phenylsulfonyl)-3-azabicyclo[3.2.0]hept-2-ene (4h)<smiles>O=S(=O)(c1ccccc1)C12C=NCC1[C@H](c1ccccc1)C2</smiles>

The general procedure (GP-3) was followed using $3 \mathrm{~h}(32 \mathrm{mg}, 0.10 \mathrm{mmol}$ ). Purification by chromatography on silica gel ( $n$-hexanes/EtOAc/DCM 50:40:10) yielded $\mathbf{4 h}(17,9 \mathrm{mg}, 56 \%)$ as a pale yellow solid. ${ }^{1} \mathbf{H} \mathbf{~ N M R}\left(300 \mathbf{~ M H z}, \mathrm{CDCl}_{3}\right) \delta=7.97-7.84(\mathrm{~m}, 2 \mathrm{H}), 7.79-7.53(\mathrm{~m}, 4 \mathrm{H}), 7.45$ $-7.20(\mathrm{~m}, 5 \mathrm{H}), 4.00(\mathrm{~d}, J=17.0 \mathrm{~Hz}, 1 \mathrm{H}), 3.53(\mathrm{ddd}, J=17.0,6.3,2.9 \mathrm{~Hz}, 1 \mathrm{H}), 3.36(\mathrm{t}, J=6.4 \mathrm{~Hz}$, $1 \mathrm{H}), 3.24(\mathrm{dd}, J=12.1,8.8 \mathrm{~Hz}, 1 \mathrm{H}), 2.97(\mathrm{td}, J=8.6,6.6 \mathrm{~Hz}, 1 \mathrm{H}), 2.72(\mathrm{dd}, J=12.1,8.5 \mathrm{~Hz}, 1 \mathrm{H})$. ${ }^{13} \mathrm{C} \mathrm{NMR}\left(75 \mathrm{MHz}, \mathrm{CDCl}_{3}\right) \delta=161.3(\mathrm{CH}), 142.1(\mathrm{Cq}), 136.4(\mathrm{Cq}), 134.3(\mathrm{CH}), 129.4(\mathrm{CH}), 128.9$ $(\mathrm{CH}), 128.8(\mathrm{CH}), 127.3(\mathrm{CH}), 127.0(\mathrm{CH}), 76.0(\mathrm{Cq}), 67.9\left(\mathrm{CH}_{2}\right), 47.2(\mathrm{CH}), 40.4(\mathrm{CH}), 33.4\left(\mathrm{CH}_{2}\right)$. (ESI)-HRMS calcd for $\mathrm{C}_{18} \mathrm{H}_{18} \mathrm{NO}_{2} \mathrm{~S}[\mathrm{M}+\mathrm{H}]^{+} 312.1053$, found 312.1060 . 
(1R,5S,6S)-1-(mesitylsulfonyl)-6-phenyl-3-azabicyclo[3.2.0]hept-2-ene (4j)<smiles>Cc1cc(C)c(S(=O)(=O)[C@]23C=NC[C@H]2[C@@H](c2ccccc2)C3)c(C)c1</smiles>

The general procedure (GP-3) was followed using 3j $(34.6 \mathrm{mg}, 0.10 \mathrm{mmol})$. Purification by chromatography on silica gel ( $n$-hexanes/EtOAc/DCM 50:40:10) yielded $4 \mathbf{j}$ (15.8 $\mathrm{mg}, 46 \%$ ) as a dark yellow solid. ${ }^{1} \mathbf{H}$ NMR $\left(400 ~ \mathbf{~ M H}, \mathrm{CDCl}_{3}\right) \delta=7.47-7.19(\mathrm{~m}, 6 \mathrm{H}), 7.01(\mathrm{~s}, 2 \mathrm{H}), 4.14-4.05$ $(\mathrm{m}, 1 \mathrm{H}), 4.01-3.90(\mathrm{~m}, 1 \mathrm{H}), 3.53(\mathrm{t}, J=6.2 \mathrm{~Hz}, 1 \mathrm{H}), 3.20(\mathrm{dd}, J=12.0,8.9 \mathrm{~Hz}, 1 \mathrm{H}), 2.97-2.87$ (m, 1H), $2.73(\mathrm{dd}, J=12.0,8.5 \mathrm{~Hz}, 1 \mathrm{H}), 2.65(\mathrm{~s}, 6 \mathrm{H}), 2.34(\mathrm{~s}, 3 \mathrm{H}) .{ }^{13} \mathrm{C} \mathrm{NMR}\left(101 \mathrm{MHz}, \mathrm{CDCl}_{3}\right) \delta$ $=161.3(\mathrm{CH}), 144.0(\mathrm{Cq}), 142.3(\mathrm{Cq}), 140.8(\mathrm{Cq}), 132.8\left(\mathrm{CH}_{2}\right), 131.3(\mathrm{Cq}), 128.8(\mathrm{CH}), 127.1(\mathrm{CH})$, $126.9(\mathrm{CH}), 77.9(\mathrm{Cq}), 68.2\left(\mathrm{CH}_{2}\right), 48.0(\mathrm{CH}), 40.0(\mathrm{CH}), 35.44\left(\mathrm{CH}_{2}\right), 23.70\left(\mathrm{CH}_{3}\right), 21.05\left(\mathrm{CH}_{3}\right)$. (ESI)-HRMS calcd for $\mathrm{C}_{21} \mathrm{H}_{24} \mathrm{NO}_{2} \mathrm{~S}[\mathrm{M}+\mathrm{H}]^{+} 354.1522$, found 354.1532 .

(1R,5S,6S)-1-((4-nitrophenyl)sulfonyl)-6-phenyl-3-azabicyclo[3.2.0]hept-2-ene (4k)<smiles>O=[N+]([O-])c1ccc(S(=O)(=O)[C@]23C=NC[C@H]2[C@H](c2ccccc2)C3)cc1</smiles>

The general procedure (GP-3) was followed using 3k (36.6 mg, $0.10 \mathrm{mmol}$ ). Purification by chromatography on silica gel ( $n$-hexanes/EtOAc/DCM 50:40:10) yielded $\mathbf{4 k}(10.0 \mathrm{mg}, 27 \%)$ as a yellow solid. ${ }^{1} \mathrm{H}$ NMR $\left(400 \mathrm{MHz}, \mathrm{CDCl}_{3}\right) \delta=8.41(\mathrm{~d}, J=8.8 \mathrm{~Hz}, 2 \mathrm{H}), 8.08(\mathrm{~d}, J=8.8 \mathrm{~Hz}, 2 \mathrm{H})$, $7.62(\mathrm{~d}, J=2.7 \mathrm{~Hz}, 1 \mathrm{H}), 7.44-7.22(\mathrm{~m}, 5 \mathrm{H}), 4.04(\mathrm{~d}, J=17.2 \mathrm{~Hz}, 1 \mathrm{H}), 3.56$ (ddd, $J=17.3,6.4$, $2.9 \mathrm{~Hz}, 1 \mathrm{H}$ ), $3.38(\mathrm{t}, J=6.5 \mathrm{~Hz}, 1 \mathrm{H}), 3.25(\mathrm{dd}, J=12.3,8.9 \mathrm{~Hz}, 1 \mathrm{H}), 3.00(\mathrm{td}, J=8.7,6.6 \mathrm{~Hz}, 1 \mathrm{H})$, $2.74(\mathrm{dd}, J=12.3,8.6 \mathrm{~Hz}, 1 \mathrm{H}) .{ }^{13} \mathrm{C}$ NMR $(75 \mathrm{MHz}$, acetone) $\delta=159.8(\mathrm{CH}), 151.4(\mathrm{Cq}), 142.7$ (Cq), $142.3(\mathrm{Cq}), 130.7(\mathrm{CH}), 128.6(\mathrm{CH}), 126.9(\mathrm{CH}), 126.8(\mathrm{CH}), 124.6(\mathrm{CH}), 76.1(\mathrm{Cq}), 67.7$ $\left(\mathrm{CH}_{2}\right), 46.8(\mathrm{CH}), 40.1(\mathrm{CH}), 32.9\left(\mathrm{CH}_{2}\right)$. (ESI)-HRMS calcd for $\mathrm{C}_{18} \mathrm{H}_{17} \mathrm{~N}_{2} \mathrm{O}_{4} \mathrm{~S}[\mathrm{M}+\mathrm{H}]^{+} 357.0904$, found 357.0896 .

(1R,5S,6S)-1-((4-chlorophenyl)sulfonyl)-6-phenyl-3-azabicyclo[3.2.0]hept-2-ene (4i)<smiles>O=S(=O)(c1ccc(Cl)cc1)C12C=NCC1[C@H](c1ccccc1)C2</smiles>

The general procedure (GP-3) was followed using $3 \mathbf{i}(34.1 \mathrm{mg}, 0.10 \mathrm{mmol}$ ). Purification by chromatography on silica gel ( $n$-hexanes/EtOAC/DCM 50:40:10) yielded $4 \mathbf{i}$ (23.5 mg, 68\%) as a white solid. ${ }^{1} \mathrm{H}$ NMR $\left(400 \mathrm{MHz}, \mathrm{CDCl}_{3}\right) \delta=7.80(\mathrm{~d}, J=8.6 \mathrm{~Hz}, 2 \mathrm{H}), 7.61(\mathrm{~s}, 1 \mathrm{H}), 7.54(\mathrm{~d}, J=8.5$ $\mathrm{Hz}, 2 \mathrm{H}), 7.43-7.20(\mathrm{~m}, 5 \mathrm{H}), 4.01(\mathrm{~d}, J=17.1 \mathrm{~Hz}, 1 \mathrm{H}), 3.56$ (ddd, $J=17.2,6.3,2.9 \mathrm{~Hz}, 1 \mathrm{H}), 3.33$ $(\mathrm{t}, J=6.4 \mathrm{~Hz}, 1 \mathrm{H}), 3.21(\mathrm{dd}, J=12.2,8.8 \mathrm{~Hz}, 1 \mathrm{H}), 2.96(\mathrm{td}, J=8.7,6.6 \mathrm{~Hz}, 1 \mathrm{H}), 2.71(\mathrm{dd}, J=12.2$, $8.6 \mathrm{~Hz}, 1 \mathrm{H}) .{ }^{13} \mathrm{C} \mathrm{NMR}\left(75 \mathrm{MHz}, \mathrm{CDCl}_{3}\right) \delta=161.0(\mathrm{CH}), 141.9(\mathrm{Cq}), 141.3(\mathrm{Cq}), 134.9(\mathrm{Cq}), 130.3$ 
(CH), $129.8(\mathrm{CH}), 128.8(\mathrm{CH}), 127.3(\mathrm{CH}), 126.9(\mathrm{CH}), 76.1(\mathrm{Cq}), 68.0\left(\mathrm{CH}_{2}\right), 47.2(\mathrm{CH}), 40.4(\mathrm{CH})$, $33.4\left(\mathrm{CH}_{2}\right)$. (ESI)-HRMS calcd for $\mathrm{C}_{18} \mathrm{H}_{17} \mathrm{CINO}_{2} \mathrm{~S}[\mathrm{M}+\mathrm{H}]^{+} 346.0663$, found 346.0653 .

(1R,5S,6S)-1-(naphthalen-1-ylsulfonyl)-6-phenyl-3-azabicyclo[3.2.0]hept-2-ene (4I)<smiles>O=S(=O)(c1cccc2ccccc12)C12CN=CC1[C@@H](c1ccccc1)C2</smiles>

The general procedure (GP-3) was followed using 31 (72.3 $\mathrm{mg}, 0.20 \mathrm{mmol})$. Purification by chromatography on silica gel ( $n$-hexanes/EtOAc/DCM 50:40:10) yielded $4 \mathbf{1}(30.3 \mathrm{mg}, 42 \%)$ as a pale brown solid. ${ }^{1} \mathbf{H}$ NMR (300 MHz, $\left.\mathrm{CDCl}_{3}\right) \delta=8.45(\mathrm{~s}, 1 \mathrm{H}), 8.07-7.88(\mathrm{~m}, 3 \mathrm{H}), 7.83$ (dd, $J=$ 8.6, $1.9 \mathrm{~Hz}, 1 \mathrm{H}), 7.76-7.56(\mathrm{~m}, 3 \mathrm{H}), 7.40-7.20(\mathrm{~m}, 5 \mathrm{H}), 3.97(\mathrm{~d}, J=16.9 \mathrm{~Hz}, 1 \mathrm{H}), 3.54$ (ddd, J $=16.8,6.3,2.8 \mathrm{~Hz}, 1 \mathrm{H}), 3.43(\mathrm{t}, J=6.4 \mathrm{~Hz}, 1 \mathrm{H}), 3.28(\mathrm{dd}, J=12.2,8.8 \mathrm{~Hz}, 1 \mathrm{H}), 2.96(\mathrm{td}, J=8.7$, $6.5 \mathrm{~Hz}, 1 \mathrm{H}), 2.72(\mathrm{dd}, J=12.2,8.6 \mathrm{~Hz}, 1 \mathrm{H}) .{ }^{13} \mathrm{C} \mathrm{NMR}\left(75 \mathrm{MHz}^{\mathrm{CDCl}} \mathrm{CD}_{3} \delta=161.3(\mathrm{CH}), 142.2(\mathrm{Cq})\right.$, $135.5(\mathrm{Cq}), 133.5(\mathrm{Cq}), 132.1(\mathrm{Cq}), 130.9(\mathrm{CH}), 129.7(\mathrm{CH}), 129.7(\mathrm{CH}), 129.5(\mathrm{CH}), 128.8(\mathrm{CH})$, $128.0(\mathrm{CH}), 127.9(\mathrm{CH}), 127.3(\mathrm{CH}), 127.0(\mathrm{CH}), 123.1(\mathrm{CH}), 77.3(\mathrm{Cq}), 68.0\left(\mathrm{CH}_{2}\right), 47.3(\mathrm{CH}), 40.5$ (CH), $33.6\left(\mathrm{CH}_{2}\right)$. (ESI)-HRMS calcd for $\mathrm{C}_{22} \mathrm{H}_{20} \mathrm{NO}_{2} \mathrm{~S}[\mathrm{M}+\mathrm{H}]^{+} 362.1209$, found 361.1213.

\section{(1R,6R,7S)-7-phenyl-1-tosyl-3-azabicyclo[4.2.0]oct-2-ene (4m)}

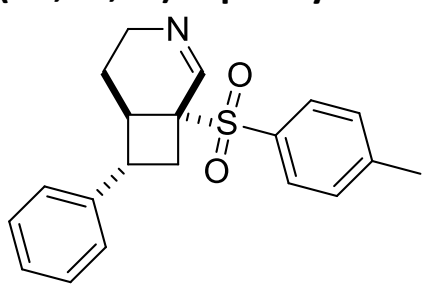

The general procedure (GP-3) was followed using $3 \mathrm{~m}(46 \mathrm{mg}, 0.13 \mathrm{mmol})$. Purification by chromatography on silica gel (gradient from $n$-hexanes/EtOAc/DCM 60:30:10 to 50:40:10) yielded $4 \mathrm{~m}(12.1 \mathrm{mg}, 26 \%)$ as a white solid. ${ }^{1} \mathrm{H}$ NMR (400 MHz, $\left.\mathrm{CDCl}_{3}\right) \delta=8.21(\mathrm{~s}, 1 \mathrm{H}), 7.75(\mathrm{~d}$, $J=8.3 \mathrm{~Hz}, 2 \mathrm{H}), 7.50-7.11(\mathrm{~m}, 8 \mathrm{H}), 3.79(\mathrm{dd}, J=18.3,5.6 \mathrm{~Hz}, 1 \mathrm{H}), 3.67-3.53(\mathrm{~m}, 1 \mathrm{H}), 3.32(\mathrm{t}$, $J=4.4 \mathrm{~Hz}, 1 \mathrm{H}), 3.21-3.06(\mathrm{~m}, 2 \mathrm{H}), 2.61-2.48(\mathrm{~m}, 1 \mathrm{H}), 2.45(\mathrm{~s}, 3 \mathrm{H}), 1.55-1.44(\mathrm{~m}, 1 \mathrm{H}), 0.95$ $-0.80(\mathrm{~m}, 1 \mathrm{H}) .{ }^{13} \mathrm{C}$ NMR (101 MHz, $\left.\mathrm{CDCl}_{3}\right) \delta=157.7(\mathrm{CH}), 145.5(\mathrm{Cq}), 142.0(\mathrm{Cq}), 133.4(\mathrm{Cq})$, 130.1 (CH), $129.2(\mathrm{CH}), 128.8(\mathrm{CH}), 127.2(\mathrm{CH}), 127.1(\mathrm{CH})(\mathrm{CH}), 56.9(\mathrm{Cq}), 45.1\left(\mathrm{CH}_{2}\right), 40.1(\mathrm{CH})$, $36.3(\mathrm{CH}), 34.0\left(\mathrm{CH}_{2}\right), 21.8\left(\mathrm{CH}_{3}\right), 19.8\left(\mathrm{CH}_{2}\right)$. (ESI)-HRMS calcd for $\mathrm{C}_{20} \mathrm{H}_{21} \mathrm{NNaO}_{2} \mathrm{~S}[\mathrm{M}+\mathrm{H}]^{+}$ 362.1185 , found 362.1191 . 


\section{X-Ray crystallography data}

Single crystal Data for 2a, 2'-1, and 4a were collected with a Bruker D8 diffractometer equipped with Photonll area detector, using a CuK $\alpha$ or a MoK $\alpha$ microfocus radiation source. Crystals were grown by vapor diffusion from hexane/acetone ( $2 a$ and $4 a)$ and hexane/THF $\left(2^{\prime}-1\right)$ and mounted on a cryoloop and the data collection strategy covered the sphere of reciprocal space. Absorption corrections were applied using the program SADABS. ${ }^{8}$ The structure was solved with the SHELXT code. ${ }^{9}$ Fourier analysis and refinement were performed by the fullmatrix least-squares methods based on F2 using SHELXL-2014 ${ }^{10}$, implemented in in Olex $2^{11}$. All the non-H atoms were refined with anisotropic displacement parameters. 
Crystal data and structure refinement for $\mathbf{2 a}, \mathbf{2}^{\prime}-\mathrm{I}$, and $\mathbf{4 a}$

\begin{tabular}{|c|c|c|c|}
\hline & $2 a$ & $2 \prime-1$ & $4 a$ \\
\hline Empirical formula & $\mathrm{C}_{20} \mathrm{H}_{21} \mathrm{NO}_{2} \mathrm{~S}$ & $\mathrm{C}_{26} \mathrm{H}_{25} \mathrm{NO}_{2} \mathrm{~S}$ & $\mathrm{C}_{19} \mathrm{H}_{19} \mathrm{NO}_{2} \mathrm{~S}$ \\
\hline Formula weight & 339.44 & 415.53 & 192.25 \\
\hline Temperature/K & 190 & 110 & 200 \\
\hline Crystal system & triclinic & monoclinic & monoclinic \\
\hline Space group & $\mathrm{P}-1$ & $\mathrm{P} 2_{1} / \mathrm{n}$ & $\mathrm{P} 2{ }_{1} / \mathrm{n}$ \\
\hline $\mathrm{a} / \AA$ & $6.0300(11)$ & $12.4094(10)$ & $5.4523(5)$ \\
\hline $\mathrm{b} / \AA ̊ \AA$ & $14.762(3)$ & $8.9350(8)$ & $10.5750(10)$ \\
\hline$c / \AA ̊$ & $29.542(7)$ & $19.7043(17)$ & $29.125(3)$ \\
\hline$\alpha /^{\circ}$ & $96.731(5)$ & 90 & 90 \\
\hline$\beta /^{\circ}$ & $93.220(5)$ & $105.200(3)$ & $95.261(3)$ \\
\hline$\gamma / /^{\circ}$ & $91.700(6)$ & 90 & 90 \\
\hline Volume $/ \AA^{3}$ & $2605.6(10)$ & 2108.3(3) & $1672.2(3)$ \\
\hline Z & 6 & 4 & 4 \\
\hline$\rho_{\text {calc }} g / \mathrm{cm}^{3}$ & 1.298 & 1.309 & 0.191 \\
\hline$\mu / \mathrm{mm}^{-1}$ & 0.198 & 0.177 & 0.380 \\
\hline$F(000)$ & 1080.0 & 880.0 & 101.0 \\
\hline Crystal size $/ \mathrm{mm}^{3}$ & $0.25 \times 0.19 \times 0.11$ & $0.12 \times 0.08 \times 0.02$ & $0.24 \times 0.23 \times 0.12$ \\
\hline Radiation & $\operatorname{MoK} \alpha(\lambda=0.71073)$ & $\operatorname{MoK} \alpha(\lambda=0.71073)$ & CuK $\alpha(\lambda=1.54184)$ \\
\hline $2 \Theta$ range for data coll. $/^{\circ}$ & 5.562 to 52.04 & 4.47 to 51.566 & 6.094 to 149.08 \\
\hline Index ranges & $\begin{array}{l}-7 \leq h \leq 7,-18 \leq k \leq 18 \\
-36 \leq \mathrm{l} \leq 36\end{array}$ & $\begin{array}{l}-15 \leq h \leq 13,-10 \leq k \leq \\
10,-24 \leq 1 \leq 24\end{array}$ & $\begin{array}{l}-6 \leq h \leq 6,-13 \leq k \leq 13,-36 \\
\leq l \leq 33\end{array}$ \\
\hline Reflections collected & 69975 & 33876 & 50378 \\
\hline Independent reflections & $\begin{array}{l}10235\left[R_{\text {int }}=0.0629\right. \\
\left.R_{\text {sigma }}=0.0352\right]\end{array}$ & $\begin{array}{l}4027\left[R_{\text {int }}=0.1284\right. \\
\left.R_{\text {sigma }}=0.0674\right]\end{array}$ & $\begin{array}{l}3380\left[R_{\text {int }}=0.0511, R_{\text {sigma }}=\right. \\
0.0202]\end{array}$ \\
\hline Data/restraints/parameters & $10235 / 0 / 652$ & $4027 / 0 / 276$ & $3380 / 0 / 209$ \\
\hline Goodness-of-fit on $F^{2}$ & 1.034 & 1.084 & 1.011 \\
\hline Final $R$ indexes $[I>=2 \sigma(I)]$ & $\begin{array}{l}R_{1}=0.0438, w R_{2}= \\
0.1008\end{array}$ & $\begin{array}{l}\mathrm{R}_{1}=0.0588, \mathrm{wR}_{2}= \\
0.1319\end{array}$ & $\mathrm{R}_{1}=0.0512, w \mathrm{R}_{2}=0.1487$ \\
\hline $\begin{array}{l}\text { Largest diff. peak/hole / e } \\
\AA^{-3}\end{array}$ & $0.23 /-0.38$ & $0.43 /-0.39$ & $0.18 /-0.39$ \\
\hline
\end{tabular}



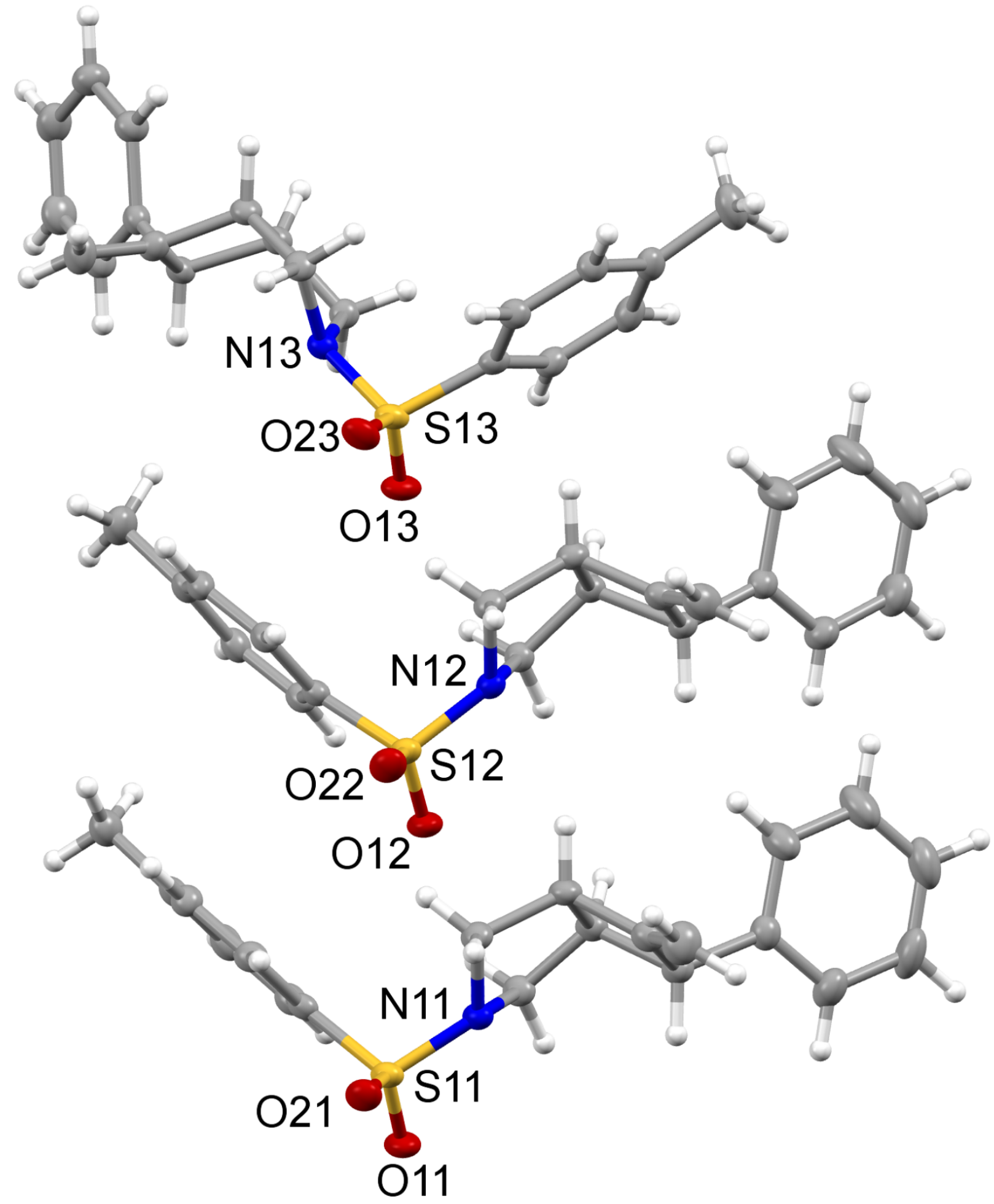

Figure S1. Molecular structure of $2 \mathrm{a}$ with thermal ellipsoids depicted at the $30 \%$ probability level. The asymmetric unit comprises three molecular entities. 


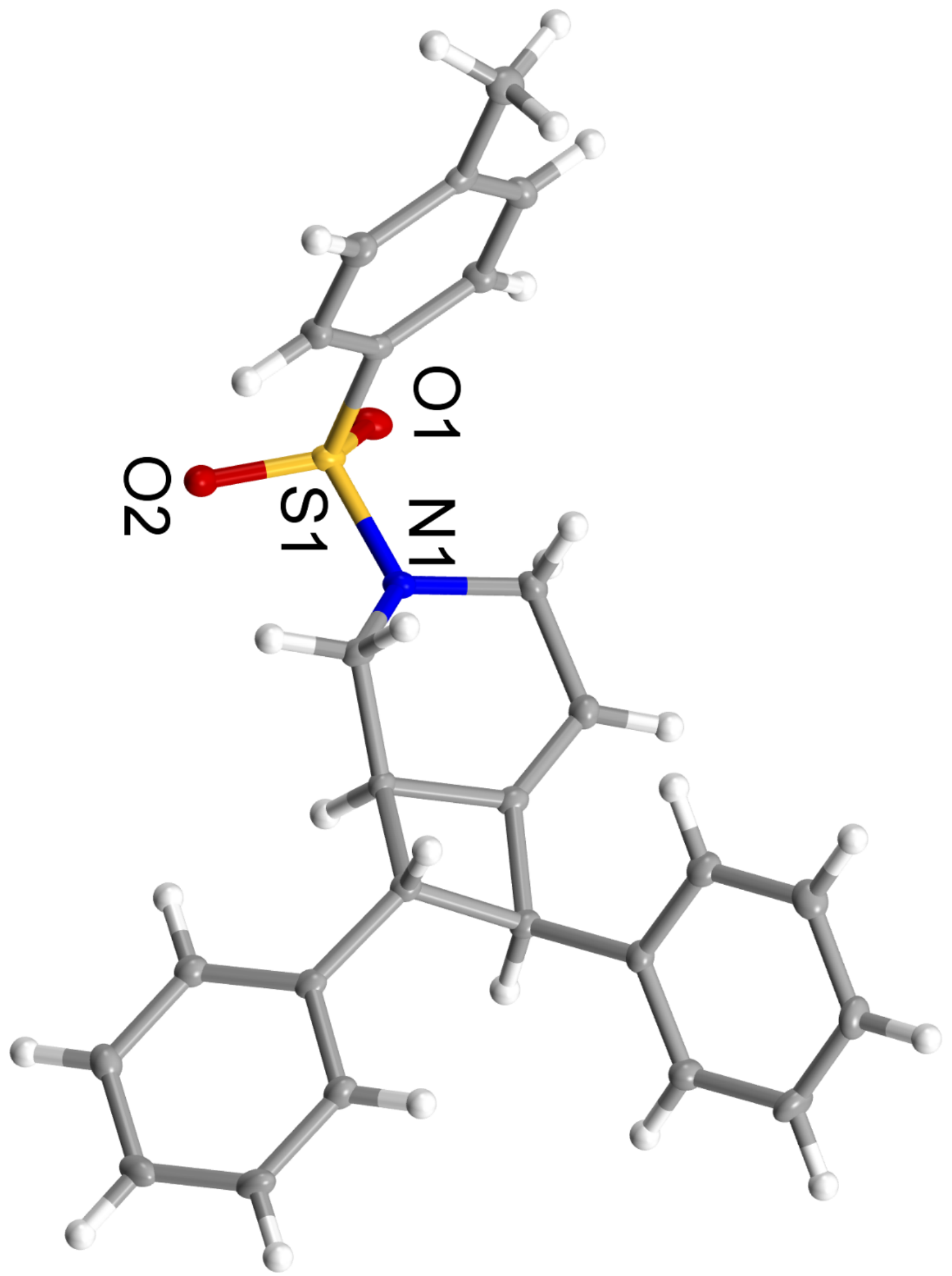

Figure S2. Molecular structure of $\mathbf{2}^{\prime}$-I with thermal ellipsoids depicted at the $30 \%$ probability level. 


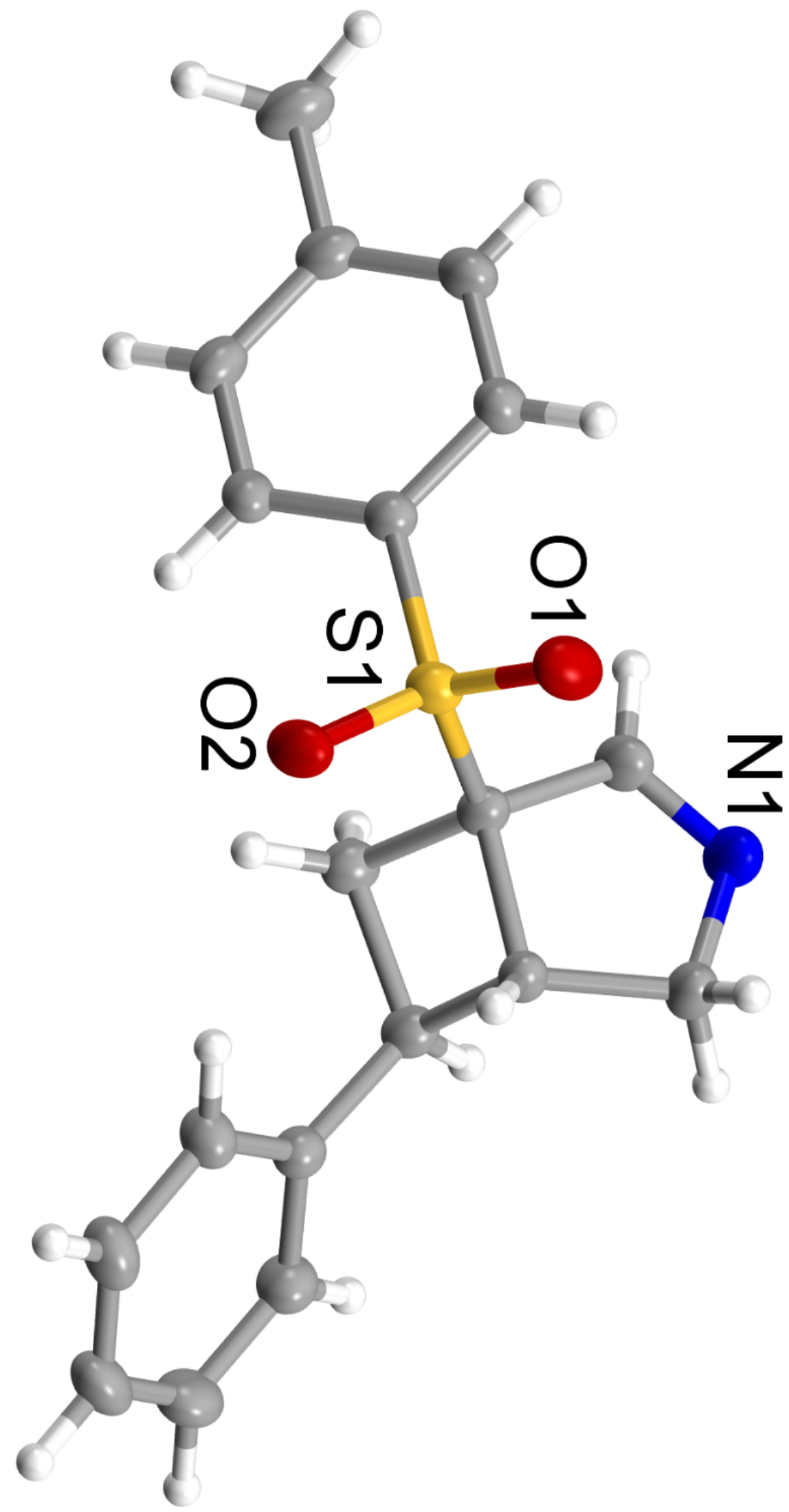

Figure S3. Molecular structure of $\mathbf{4 a}$ with thermal ellipsoids depicted at the $30 \%$ probability level. 
Copies of NMR spectra

${ }^{1} \mathrm{H}$ NMR $\left(400 \mathrm{MHz}, \mathrm{CDCl}_{3}\right)$

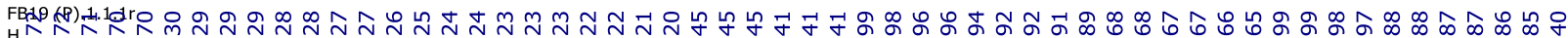

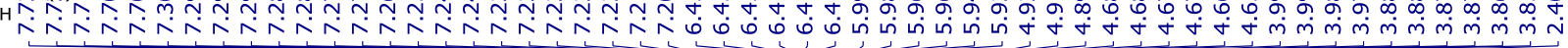
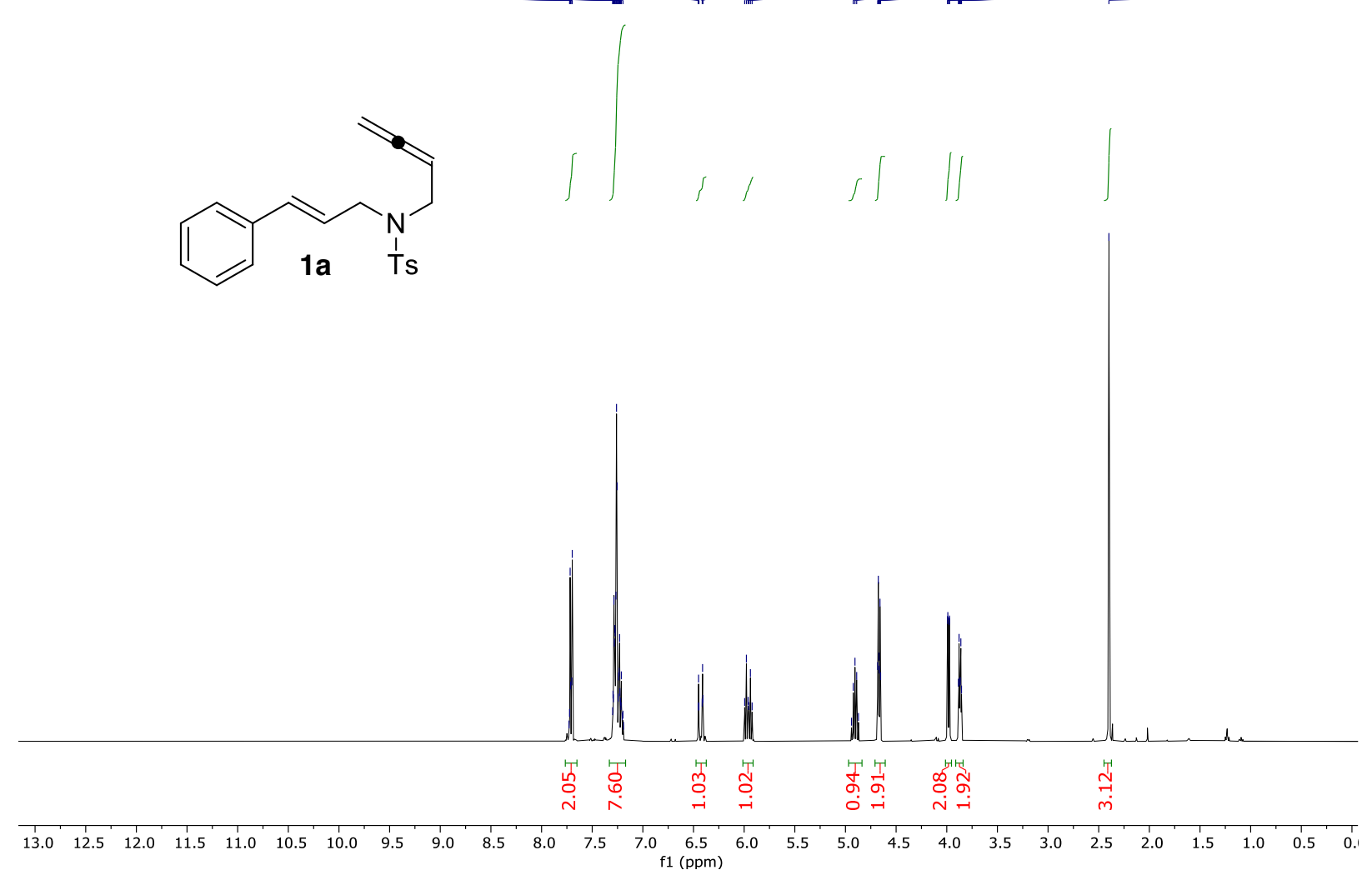

\section{${ }^{13} \mathrm{C}$ NMR (101 MHz, $\left.\mathrm{CDCl}_{3}\right)$}

FB19.(P).2.1.1r

C

i̊ m ํำ

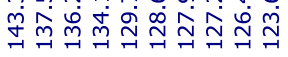

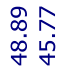

$\stackrel{\stackrel{\circ}{n}}{\stackrel{n}{N}}$

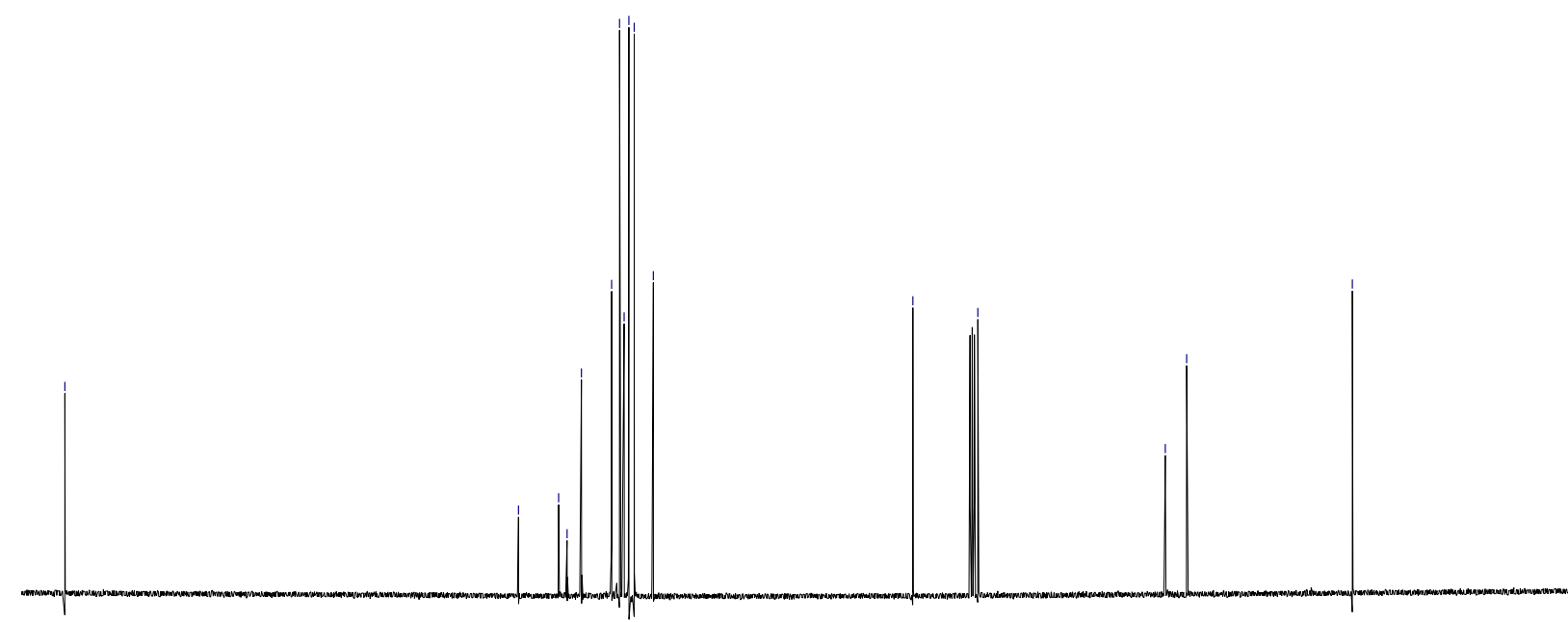

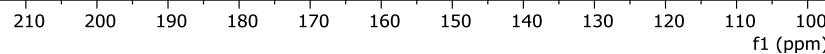


${ }^{1} \mathrm{H}$ NMR (300 MHz, acetone)

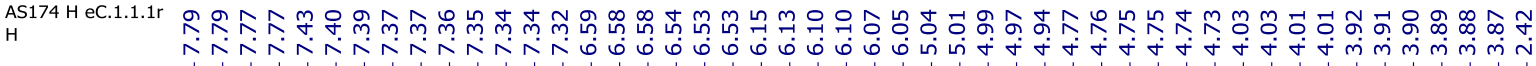<smiles>[Y9]N(C/C=C\C=C)C/C=C/c1ccc(Cl)cc1</smiles>

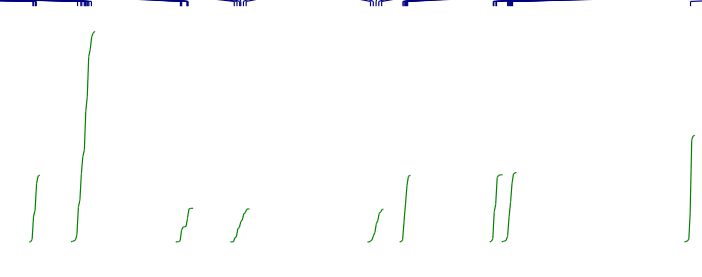

$1 b$

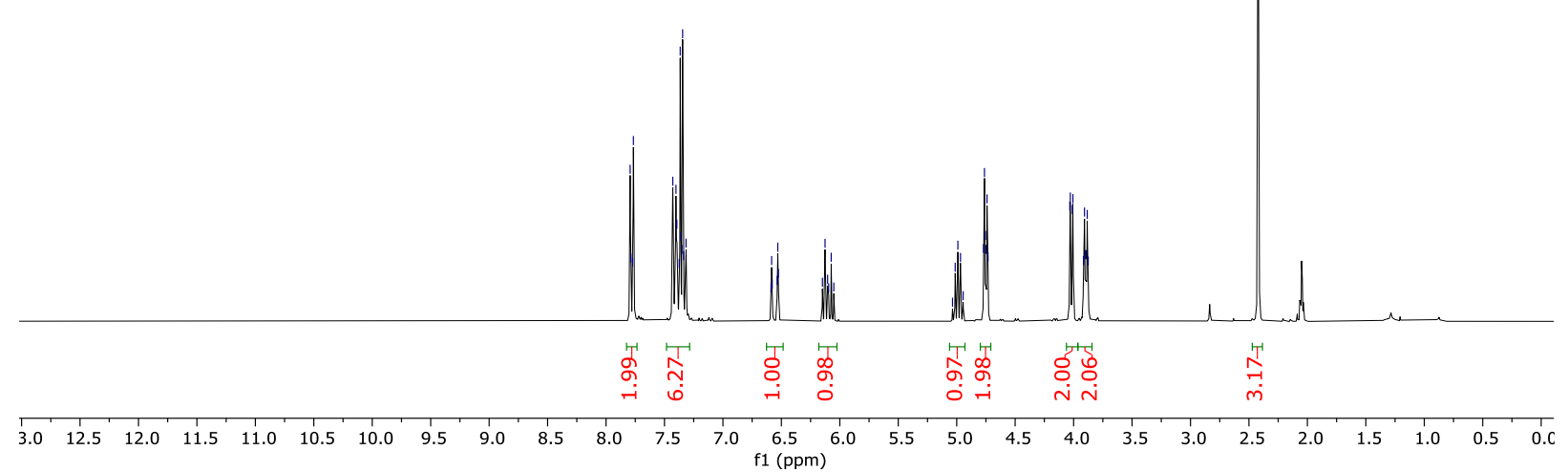

${ }^{13} \mathrm{C}$ NMR (75 MHz, acetone)

ASd 7 H ec.2.1.1r

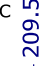

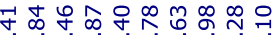

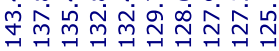

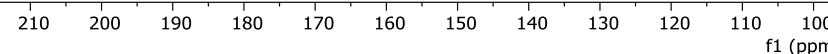

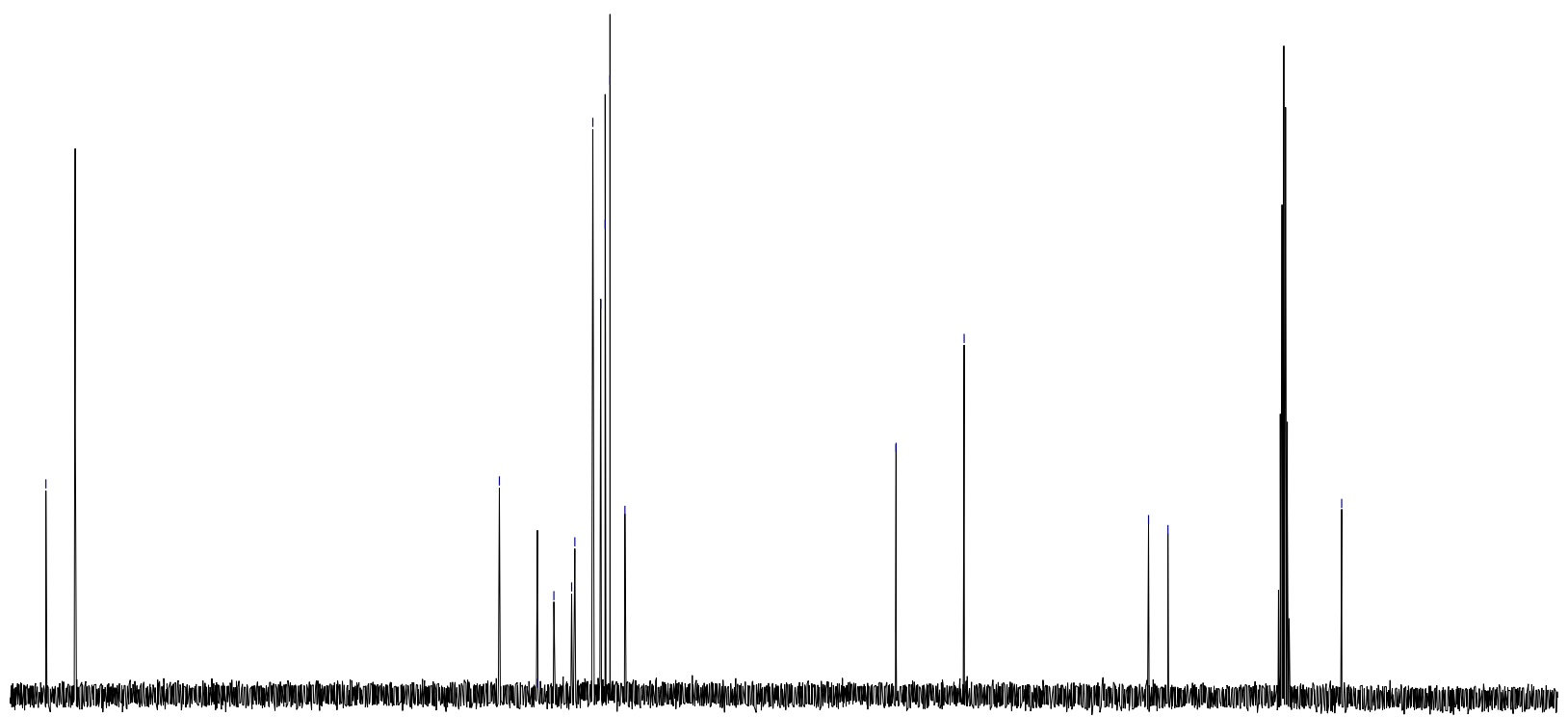

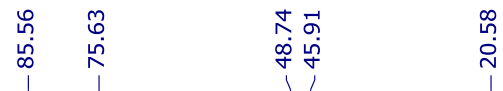

$\stackrel{\infty}{\stackrel{\infty}{N}}$ 
${ }^{1} \mathrm{H}$ NMR $\left(400 \mathrm{MHz}, \mathrm{CDCl}_{3}\right)$

AS179.1.1.11

花

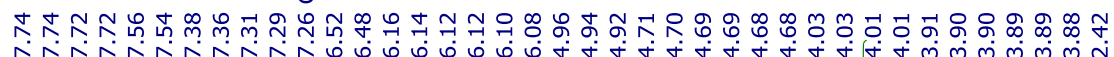

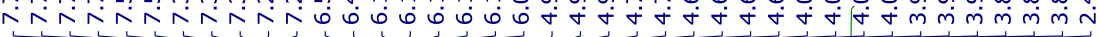<smiles>C=CCN(C)C/C=C/c1ccc(C(F)(F)F)cc1</smiles>
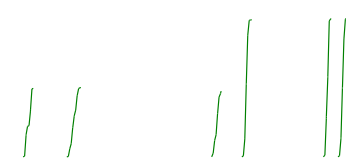

$\mathrm{F}_{3} \mathrm{C}$

Ts

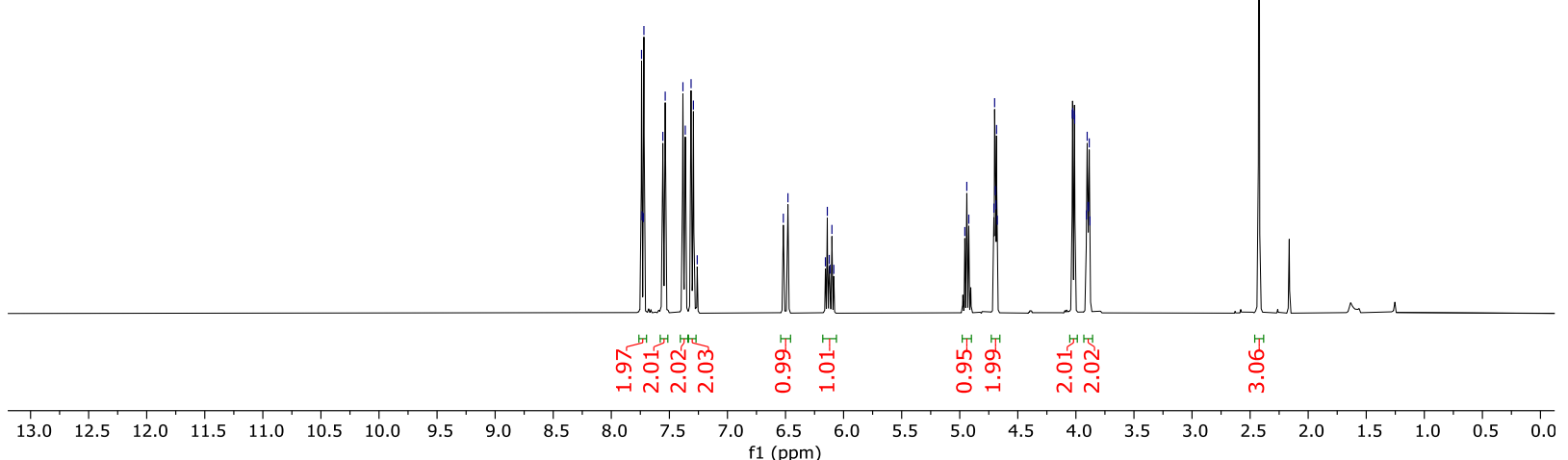

\section{${ }^{13} \mathrm{C}$ NMR (101 MHz, $\left.\mathrm{CDCl}_{3}\right)$}

$\stackrel{\circ}{\stackrel{\circ}{\circ}}$

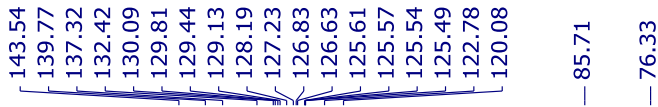

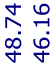

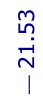

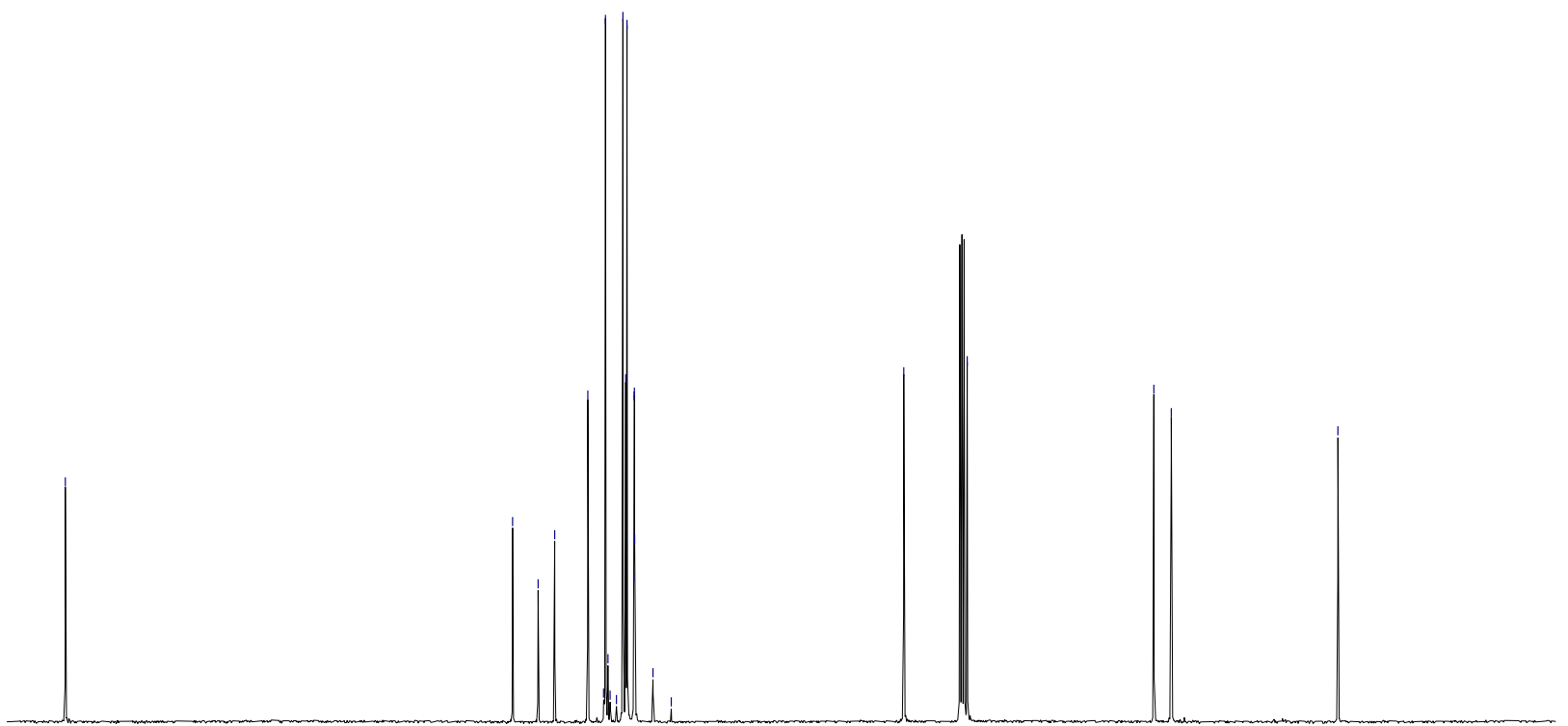

$\begin{array}{rrrrrrrrrrrrrrrrrrrrrr}1 & 1 \\ 210 & 200 & 190 & 180 & 170 & 160 & 150 & 140 & 130 & 120 & \begin{array}{r}110 \\ f 1(\mathrm{ppm})\end{array} & 90 & 80 & 70 & 60 & 50 & 40 & 30 & 20 & 10 & 0 & -1\end{array}$


${ }^{19} \mathrm{~F} \mathrm{NMR} \mathrm{(564} \mathrm{MHz,} \mathrm{CDCl}_{3}$ )

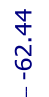<smiles>C=CCN([12CH3])C/C=C/c1ccc(C(F)(F)F)cc1</smiles>

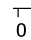

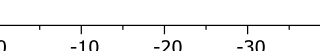

$20-100$

\begin{tabular}{llllllllll}
\hline 110 & -120 & -130 & -140 & -150 & -160 & -170 & -180 & -190 & -21
\end{tabular}


${ }^{1} \mathrm{H}$ NMR (400 MHz, $\left.\mathrm{CDCl}_{3}\right)$

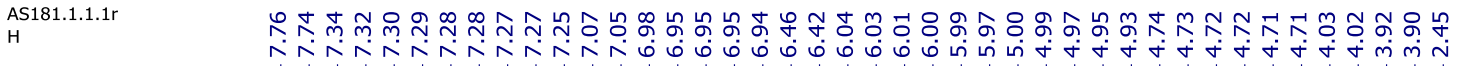<smiles>C=CCN([13CH3])C/C=C/c1cccc(F)c1</smiles>
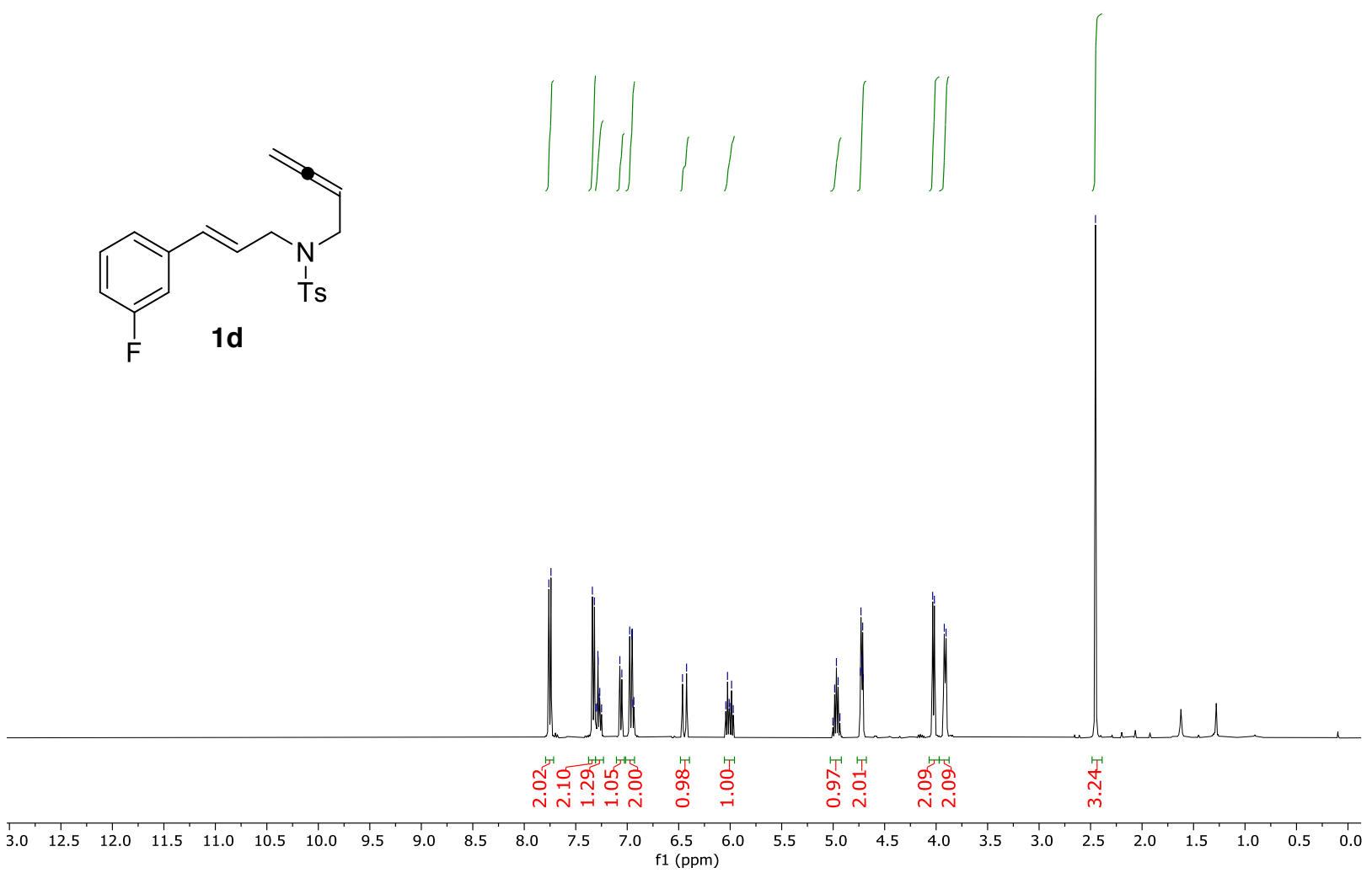

\section{${ }^{13} \mathrm{C}$ NMR (101 MHz, $\mathrm{CDCl}_{3}$ )}

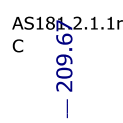

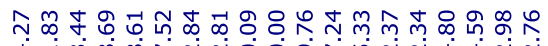

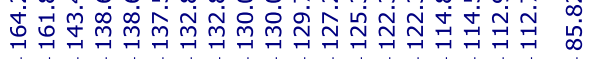

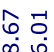

这

$\stackrel{\substack{+\stackrel{i}{i}}}{i}$

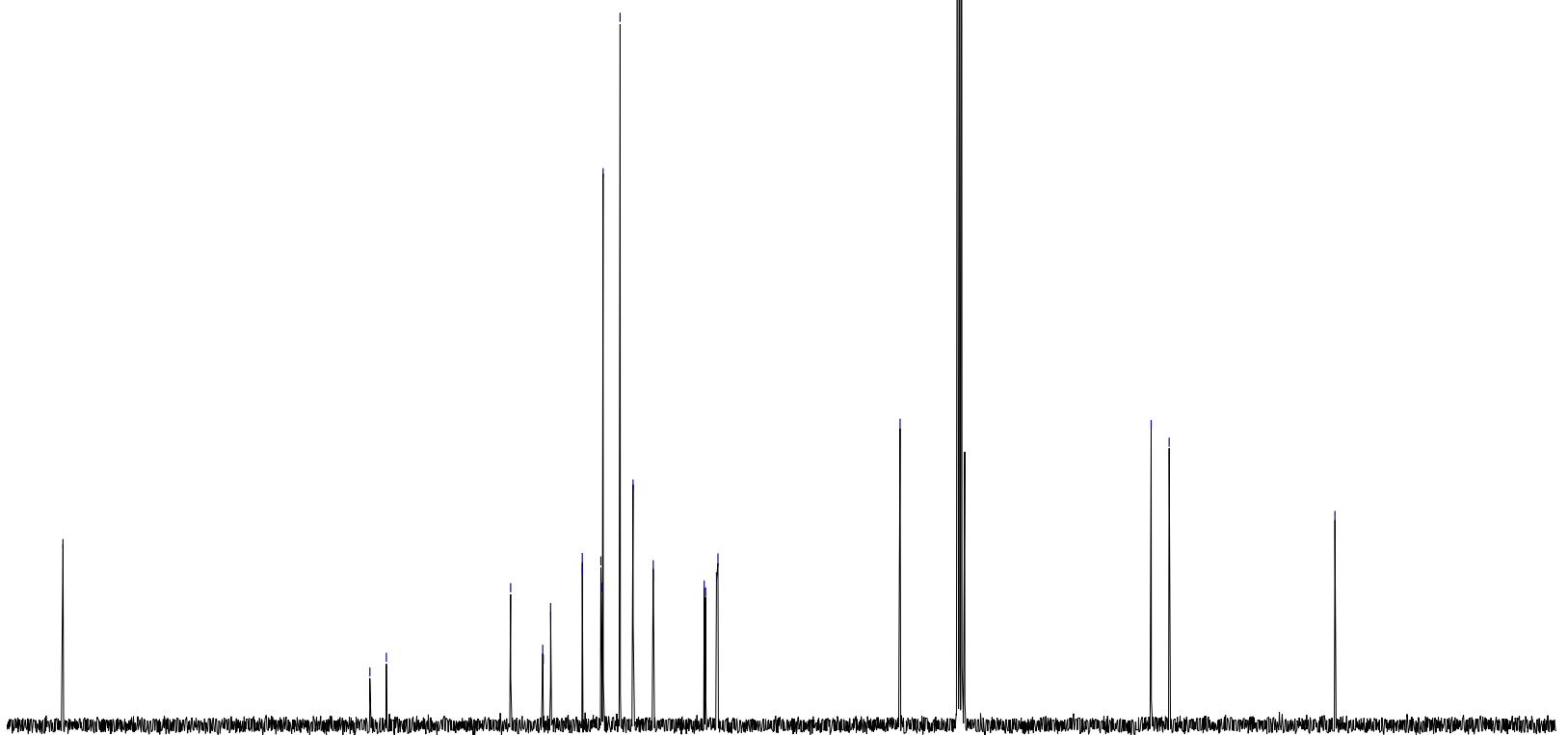

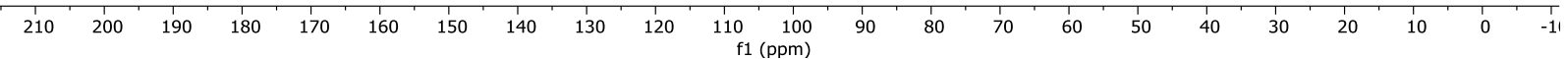


${ }^{19}$ F NMR (564 MHz, $\mathrm{CDCl}_{3}$ )

AS181
single_pulse
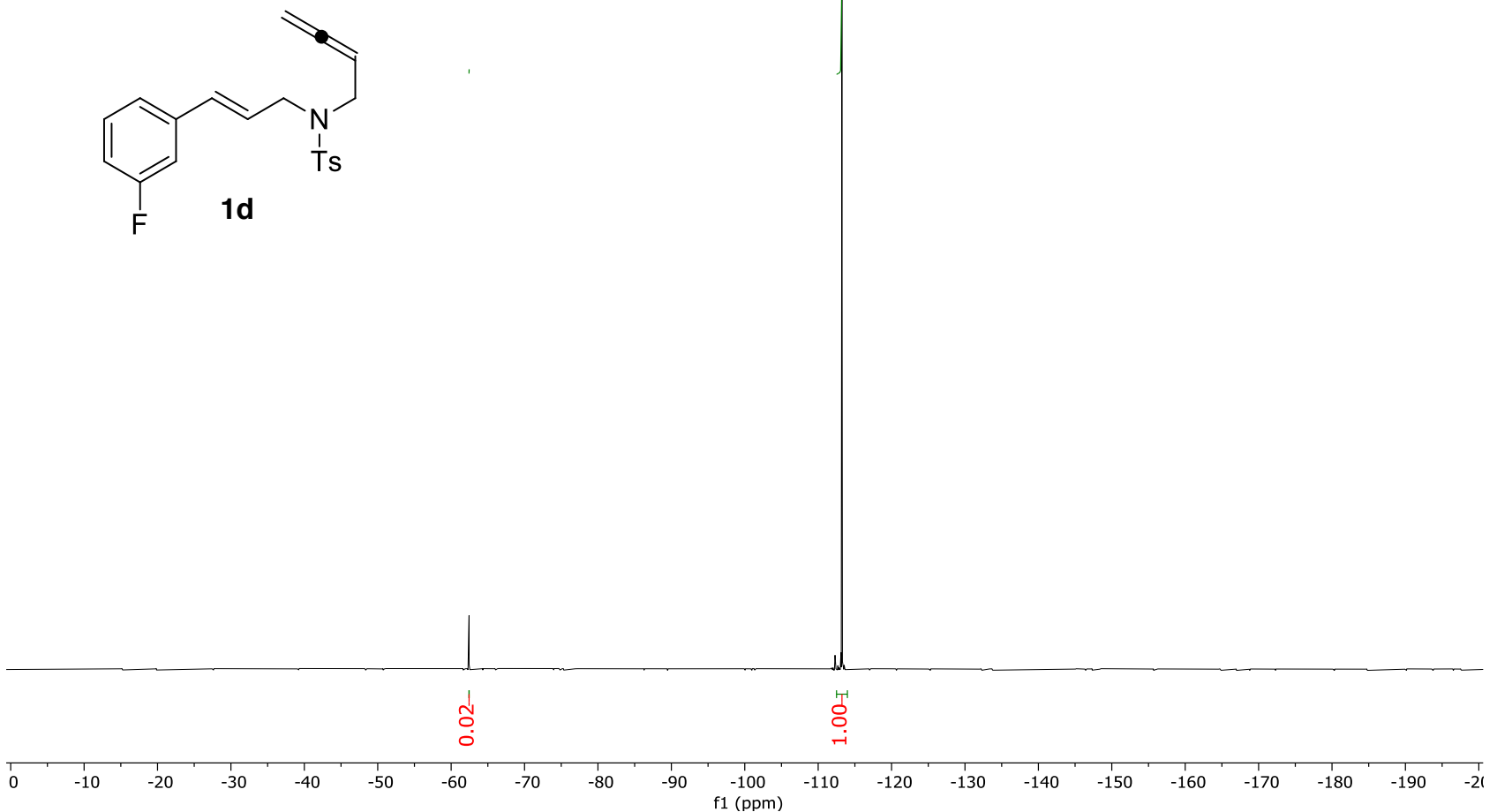
${ }^{1} \mathrm{H}$ NMR (400 MHz, $\mathrm{CDCl}_{3}$ )

AS201.2.1.1r<smiles>C=CCN(C)C/C=C/c1cccc(C#N)c1</smiles>
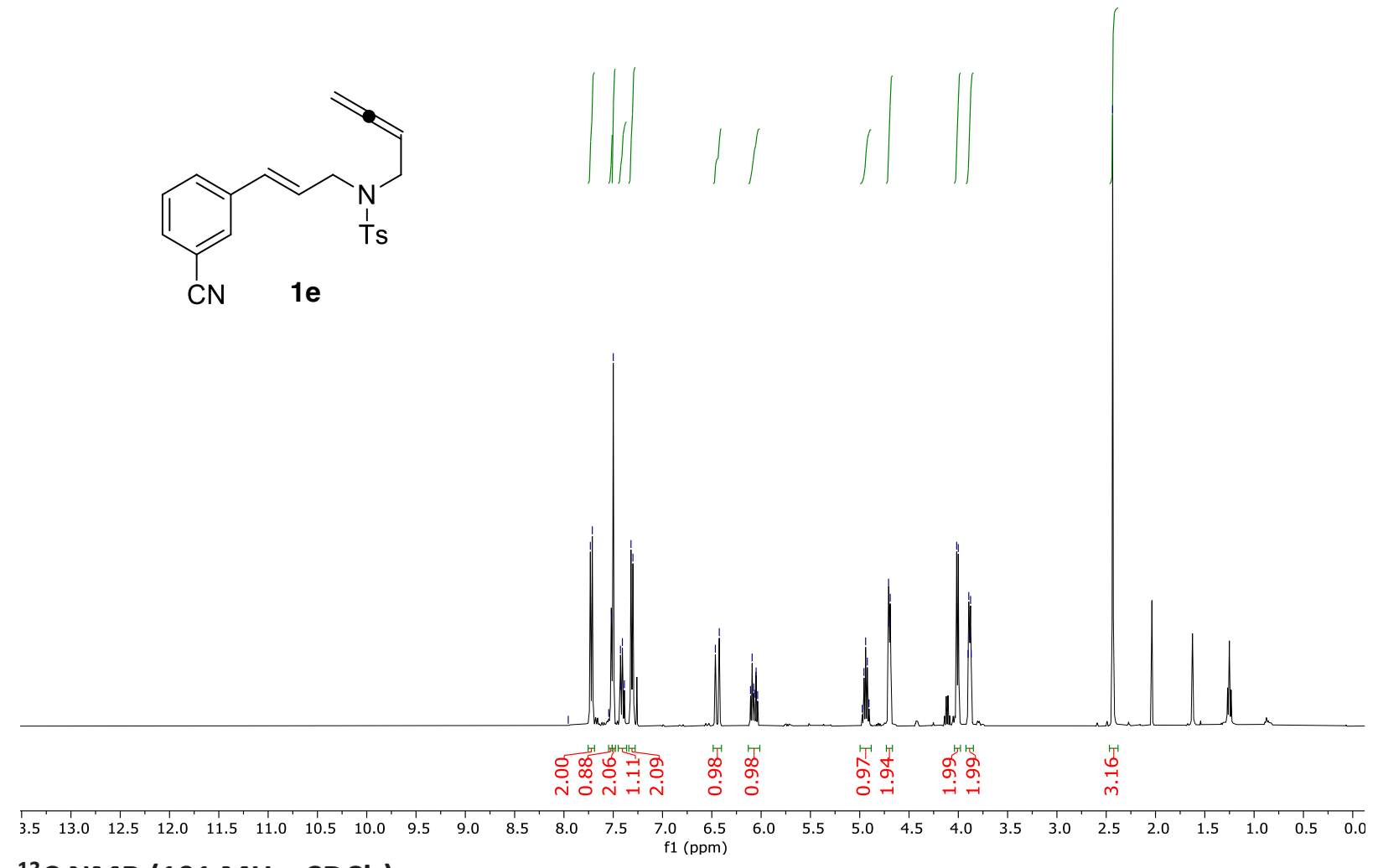

${ }^{13} \mathrm{C}$ NMR (101 $\left.\mathrm{MHz}, \mathrm{CDCl}_{3}\right)$

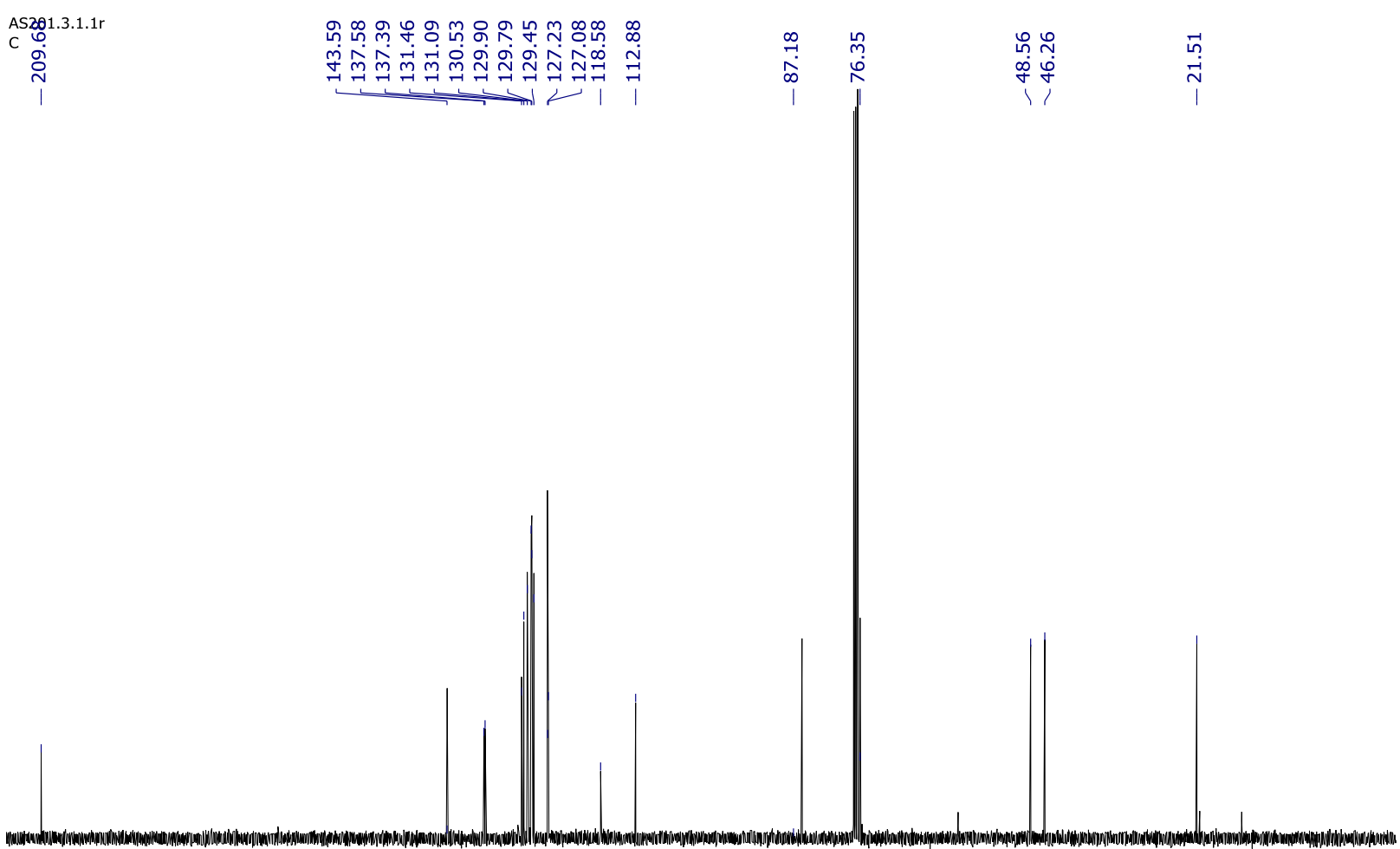

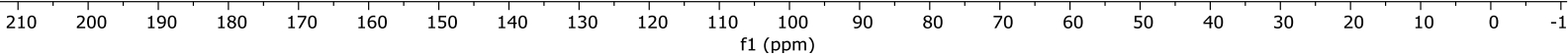


${ }^{1} \mathrm{H}$ NMR $\left(400 \mathrm{MHz}, \mathrm{CDCl}_{3}\right)$

GB15.2.1.1r

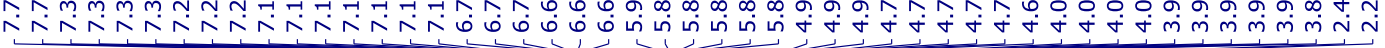

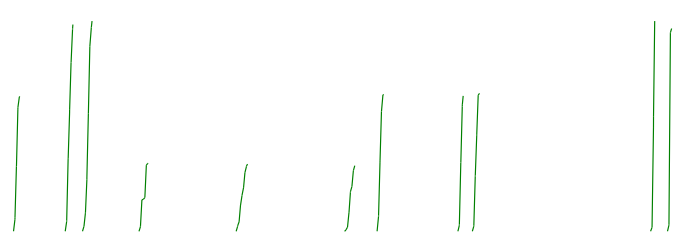<smiles>C=CCN([13F])C/C=C/c1ccccc1C</smiles>

f

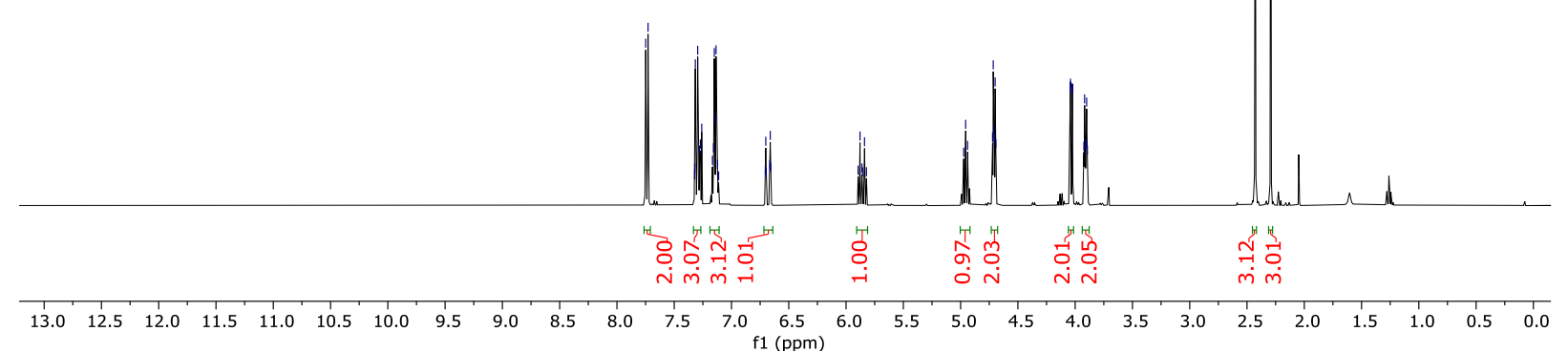

${ }^{13} \mathrm{C}$ NMR (101 MHz, $\left.\mathrm{CDCl}_{3}\right)$

GB153.1.1.

$\stackrel{\text { iे. }}{\text { i. }}$

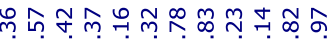

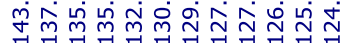

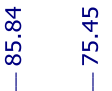

$\stackrel{\infty}{\infty}$

$\stackrel{\infty}{+} \stackrel{\sim}{+}$

นุก

국

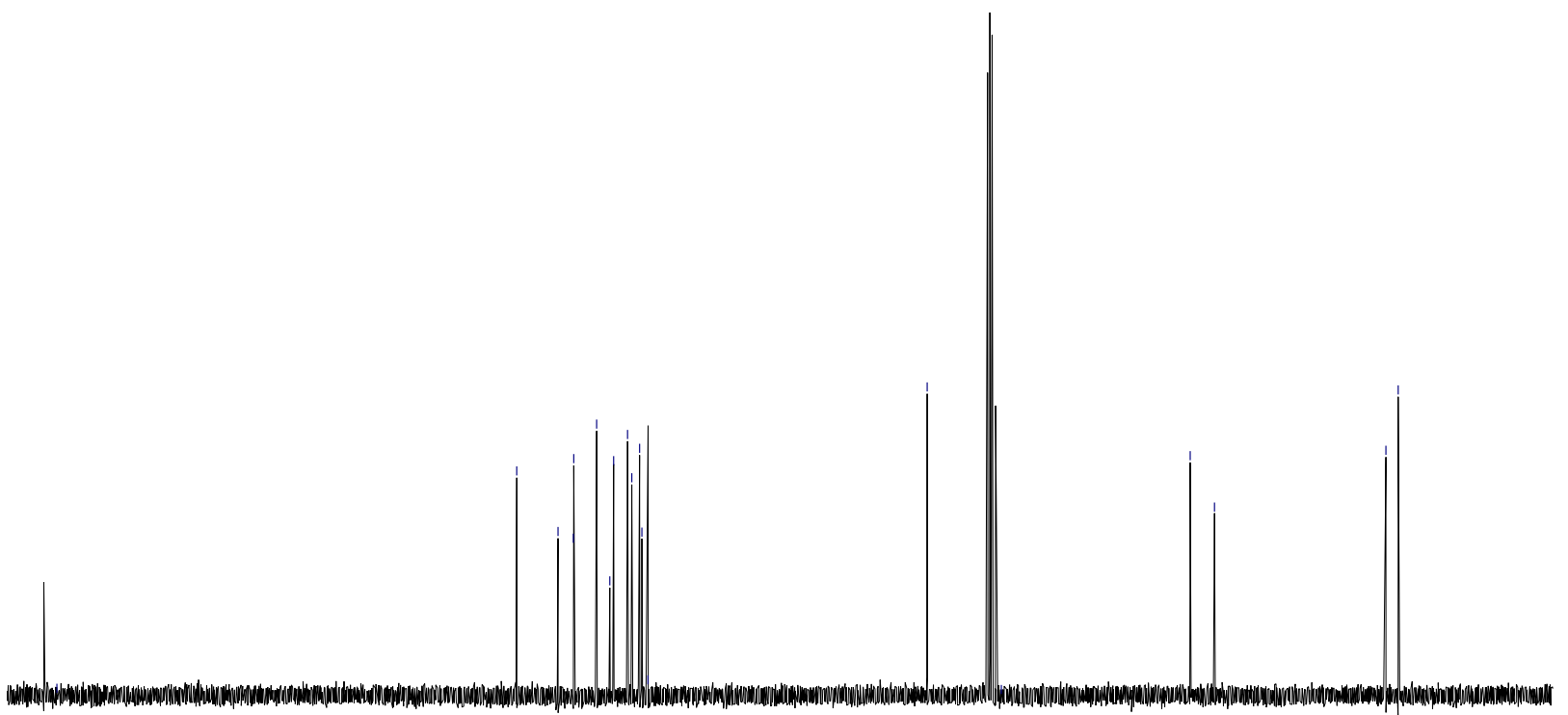

$\begin{array}{lllllllllllllllllllllll}1 & 1 & 200 & 190 & 180 & 170 & 160 & 150 & 140 & 130 & 120 & 110 & 100 & 90 & 80 & 70 & 60 & 50 & 40 & 30 & 20 & 10 & 0\end{array}$ 
${ }^{1} \mathrm{H}$ NMR $\left(400 \mathrm{MHz}, \mathrm{CDCl}_{3}\right)$

As240h,c.1fid<smiles>[Y9]N(C/C=C\C)C/C=C/c1cccnc1</smiles>
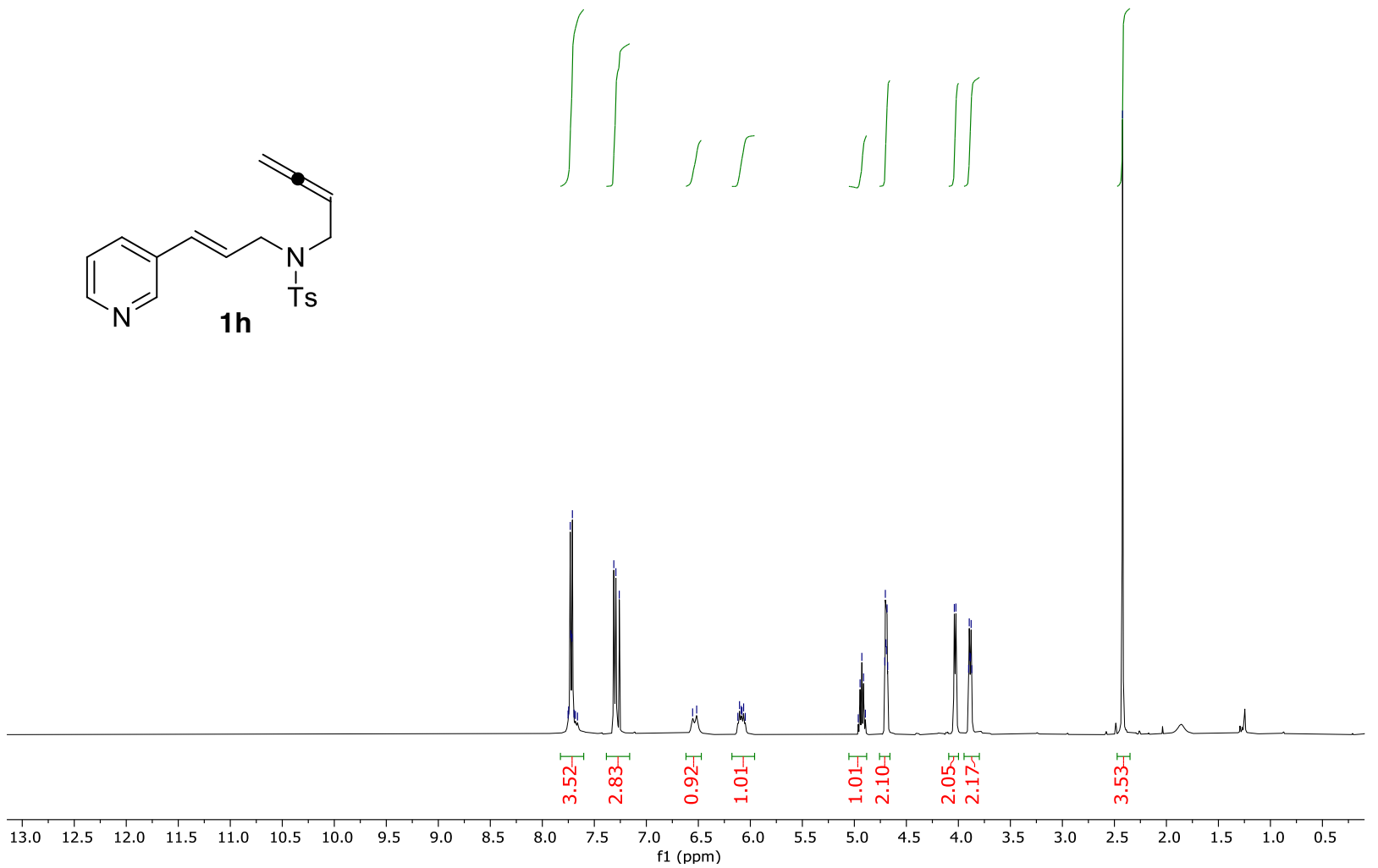

${ }^{13} \mathrm{C}$ NMR (101 MHz, $\mathrm{CDCl}_{3}$ )

AS2 24 h,c.2.1.1r

c

iे

గొน

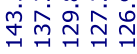

$\underset{\substack { t \\ \begin{subarray}{c}{\infty \\ \infty{ t \\ \begin{subarray} { c } { \infty \\ \infty } }\end{subarray}}{1}$

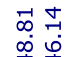

1 द

r

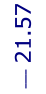

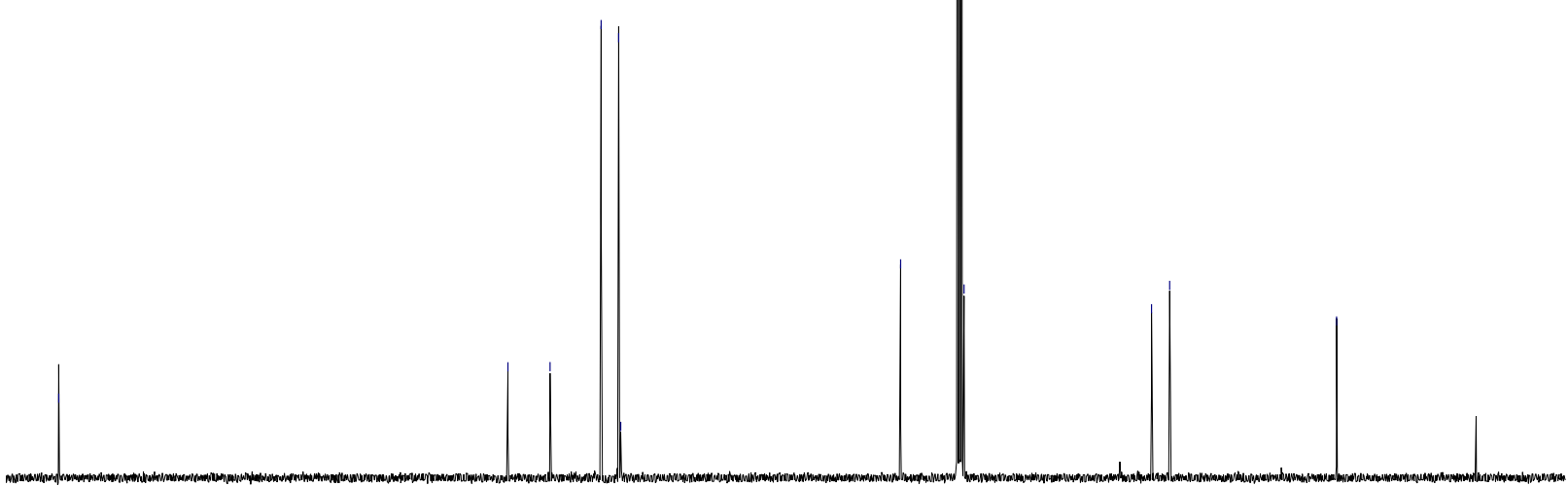

$\begin{array}{lllllllllllllllllllllll}210 & 200 & 190 & 180 & 170 & 160 & 150 & 140 & 130 & 120 & 110 & 100 & 90 & 80 & 70 & 60 & 50 & 40 & 30 & 20 & 10 & 0 & -10\end{array}$ 
${ }^{1} \mathrm{H}$ NMR (400 MHz, $\left.\mathrm{CDCl}_{3}\right)$

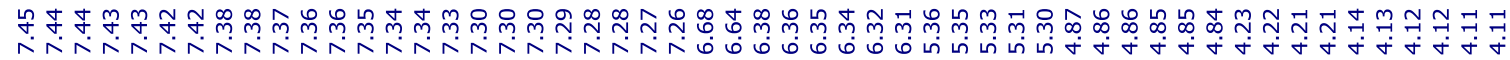<smiles>C=CCCOCC=Cc1ccccc1</smiles>

1j

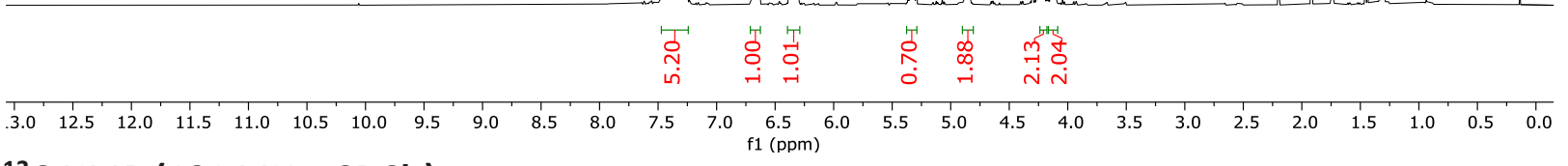

${ }^{13} \mathrm{C}$ NMR (101 MHz, $\mathrm{CDCl}_{3}$ )

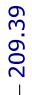

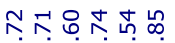

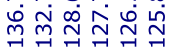

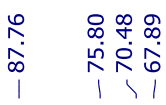

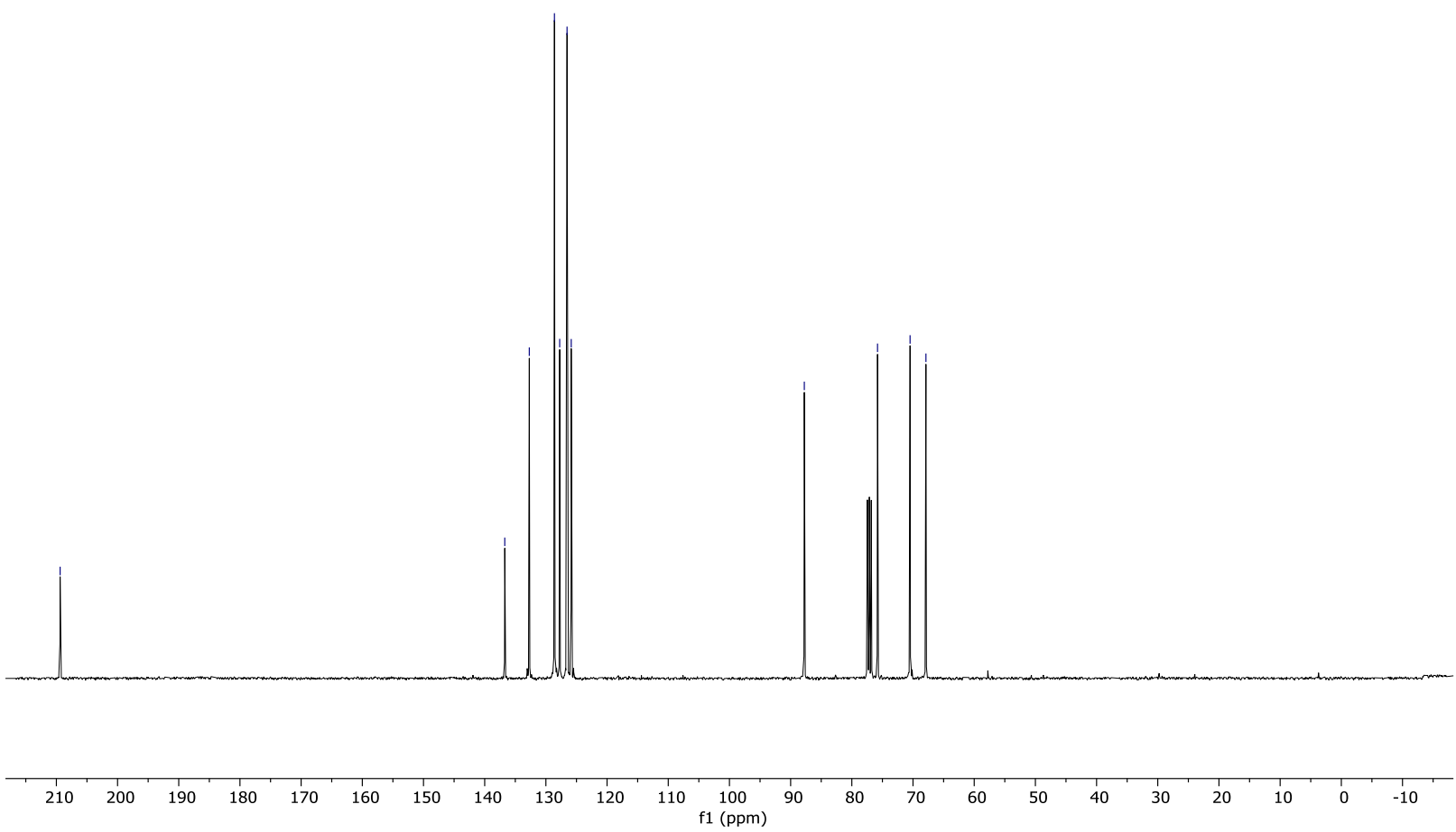


${ }^{1} \mathrm{H}$ NMR (400 MHz, $\mathrm{CDCl}_{3}$ )

等

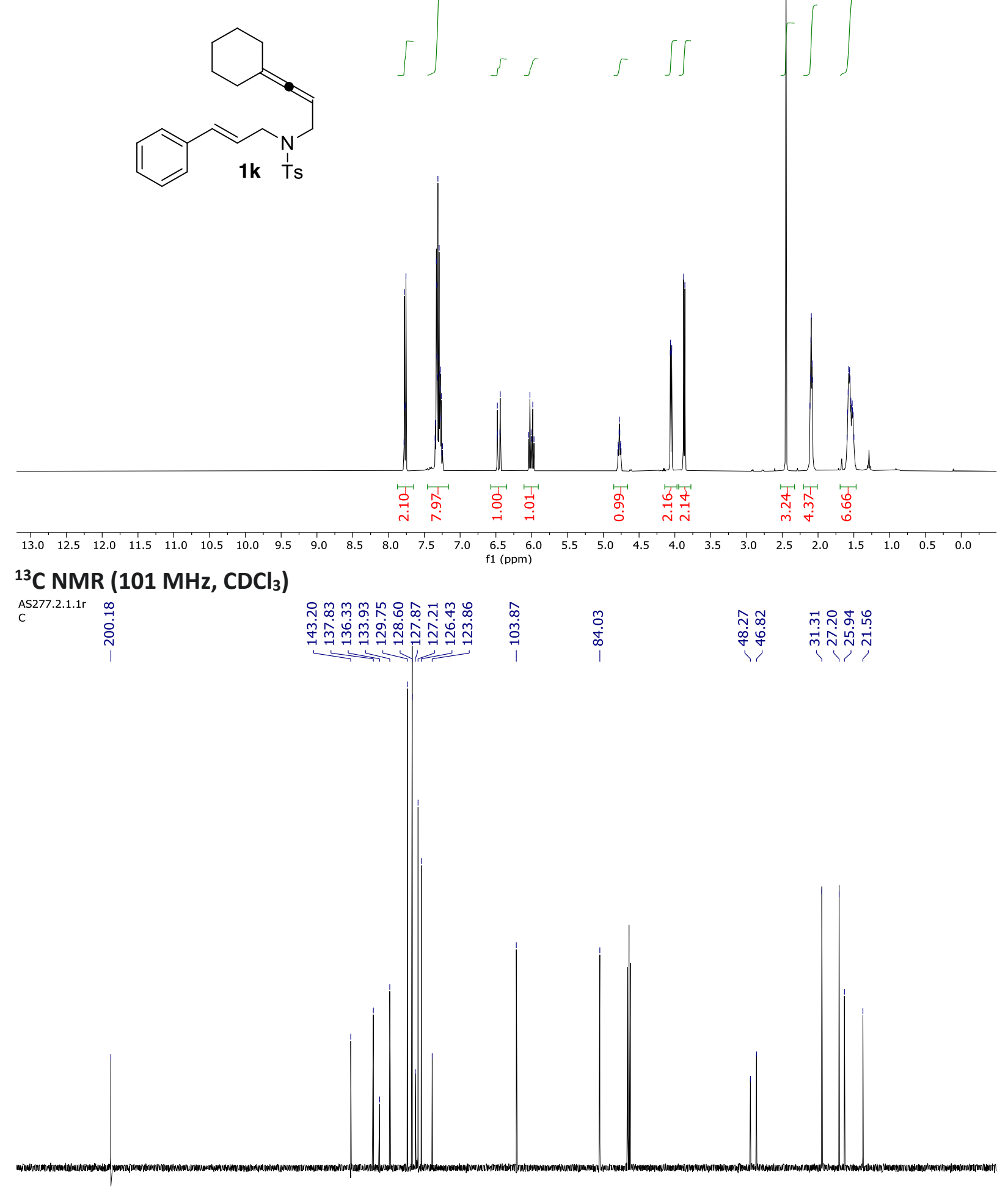

$\begin{array}{lllllllllllllllllllllllllllllll}220 & 210 & 200 & 190 & 180 & 170 & 160 & 150 & 140 & 130 & 120 & 110 & 100 & 90 & 80 & 70 & 60 & 50 & 40 & 30 & 20 & 10 & 0 & -1\end{array}$ 
${ }^{1} \mathrm{H}$ NMR (400 MHz, acetone)

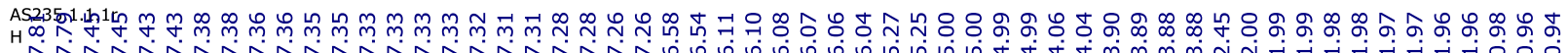

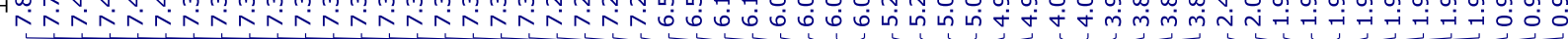
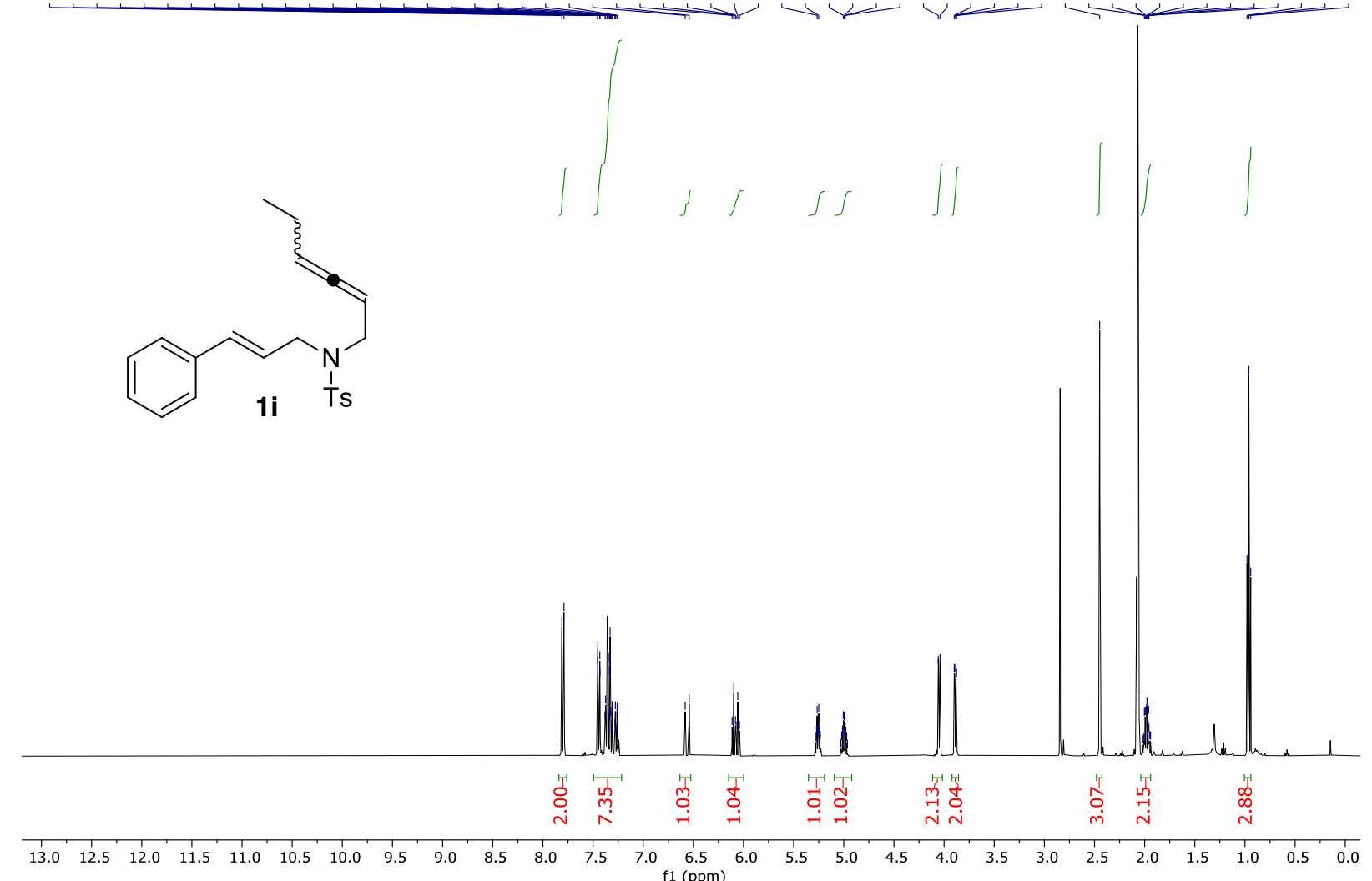

${ }^{13} \mathrm{C}$ NMR (101 MHz, acetone)

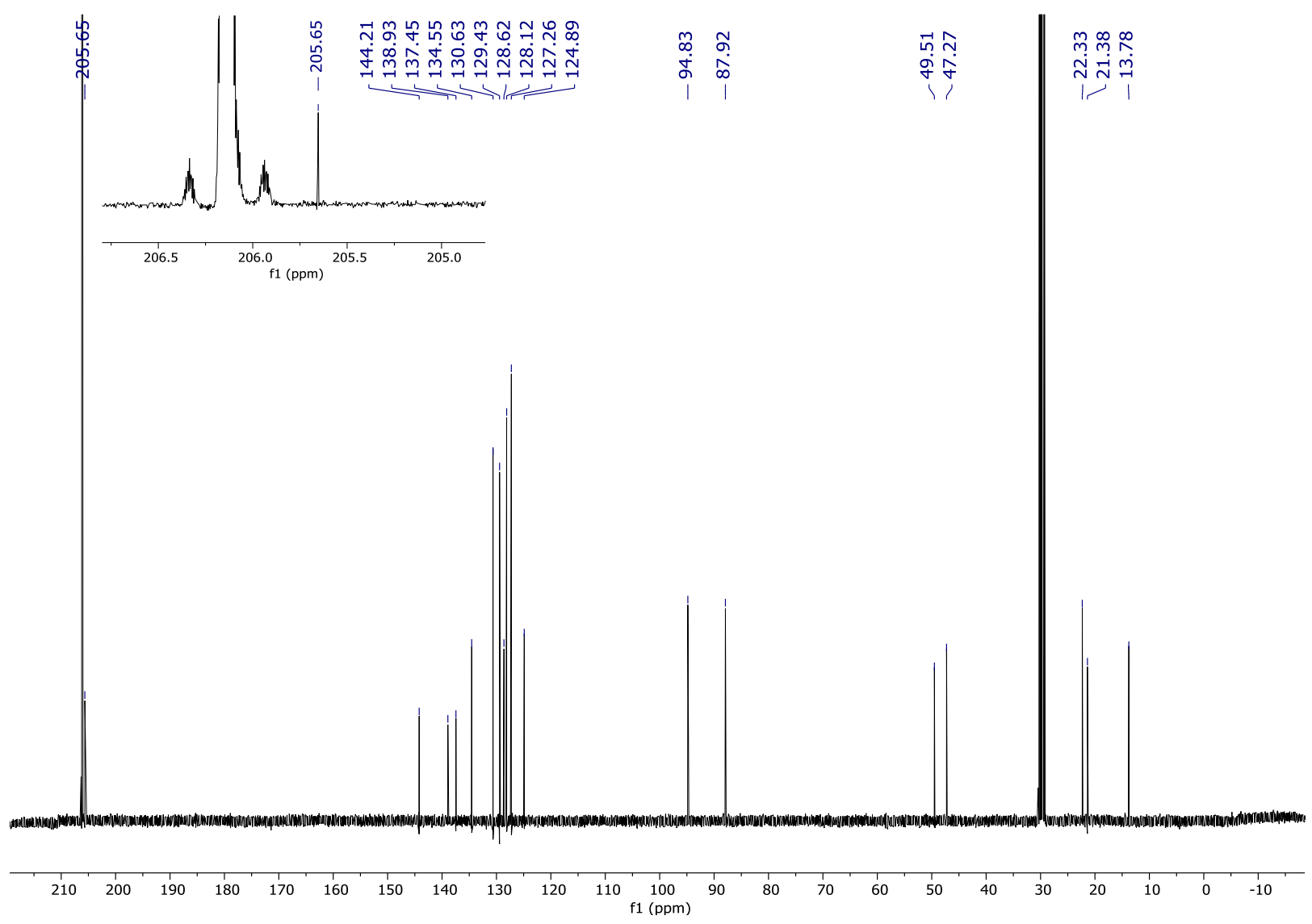


${ }^{1} \mathrm{H}$ NMR (400 MHz, $\left.\mathrm{CDCl}_{3}\right)$

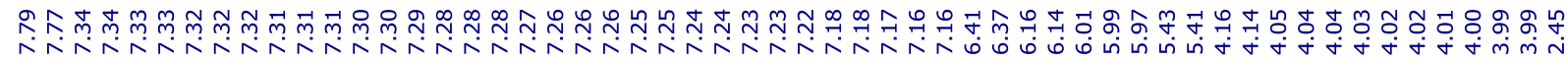<smiles>[Al]N(C/C=C/c1ccccc1)C/C=C/c1ccccc1</smiles>
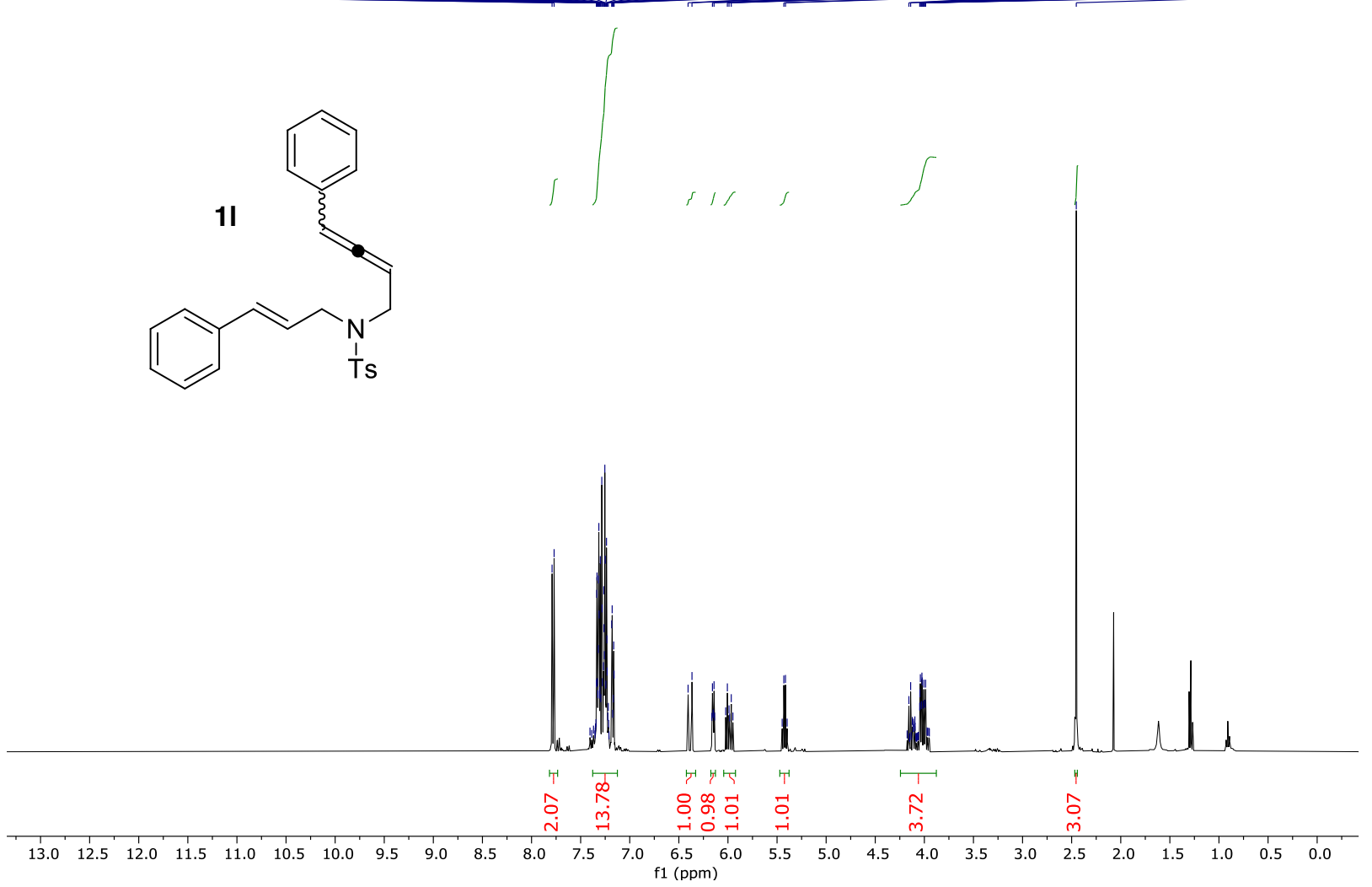

${ }^{13} \mathrm{C}$ NMR (101 $\left.\mathrm{MHz}, \mathrm{CDCl}_{3}\right)$

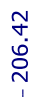

₹

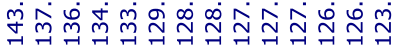

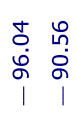

مे $\underset{\infty}{\infty}$

安

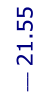

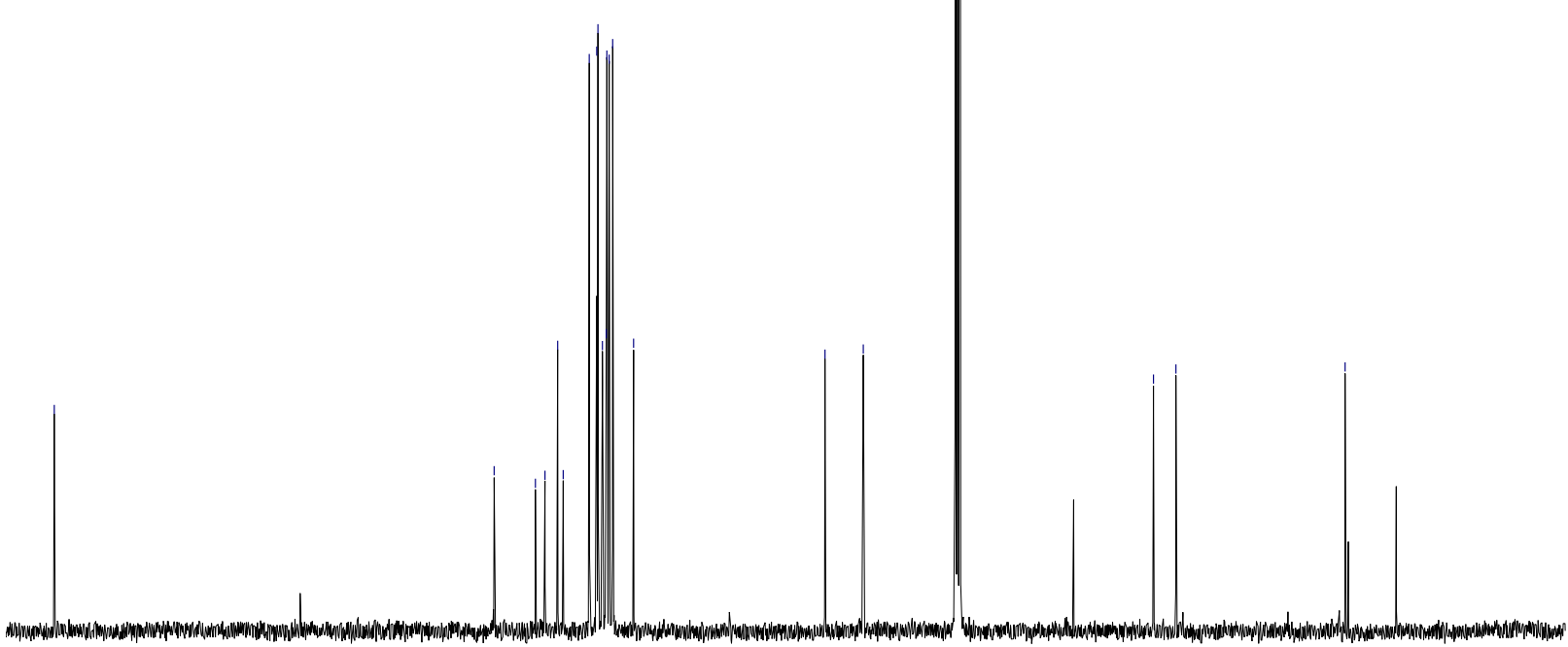

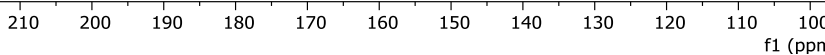


${ }^{1} \mathrm{H}$ NMR $\left(400 \mathrm{MHz}, \mathrm{CDCl}_{3}\right)$

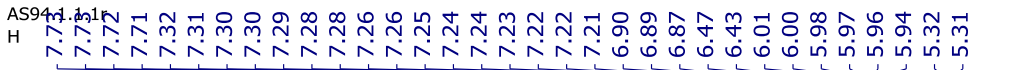

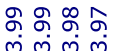
inm<smiles>C=C=CN(C/C=C/c1ccccc1)S(=O)(=O)c1ccc(C)cc1</smiles>
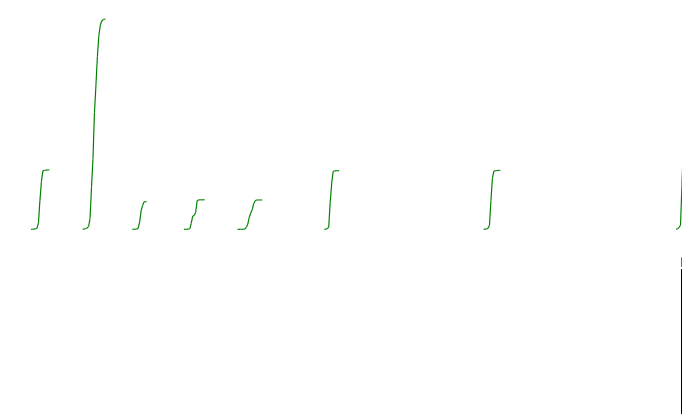

$3 a$

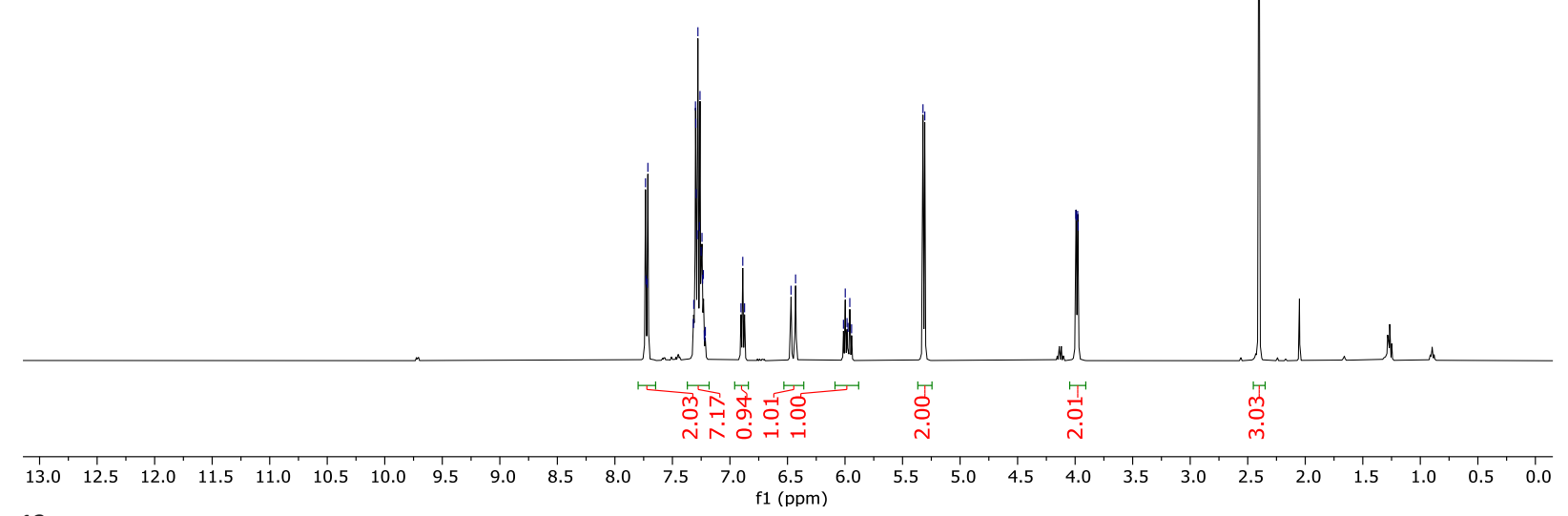

${ }^{13} \mathrm{C}$ NMR (101 MHz, $\left.\mathrm{CDCl}_{3}\right)$

AS94.2.1.1N

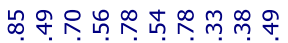

亲

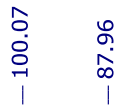

$\underset{\substack{\infty \\ \infty}}{\substack{\infty \\ \mid}}$

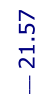

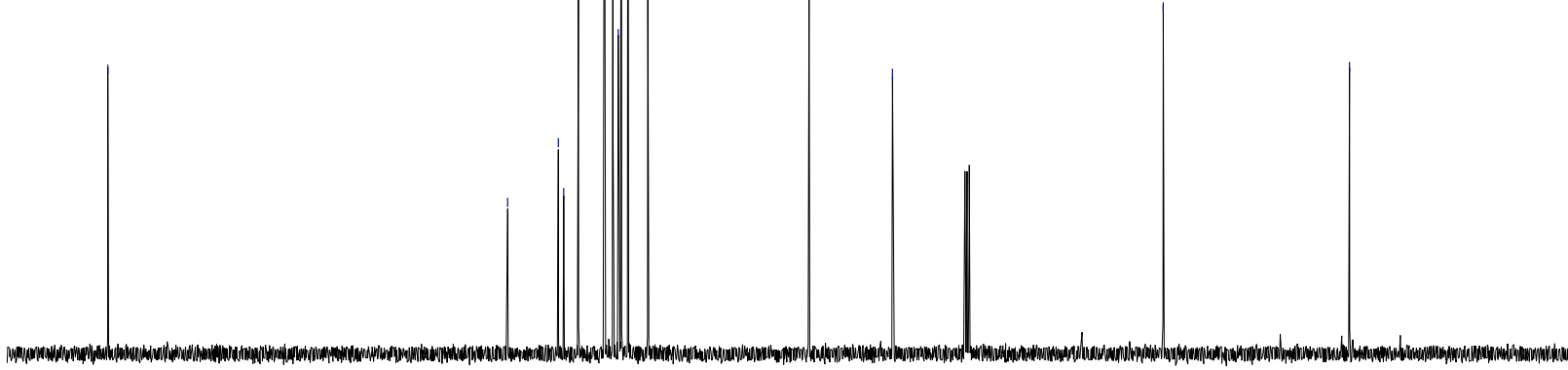

$\begin{array}{llllllllllllllllllllllll}210 & 200 & 190 & 180 & 170 & 160 & 150 & 140 & 130 & 120 & 110 & 100 & 90 & 80 & 70 & 60 & 50 & 40 & 30 & 20 & 10 & 0 & -1\end{array}$ 
${ }^{1} \mathrm{H}$ NMR (400 MHz, acetone)

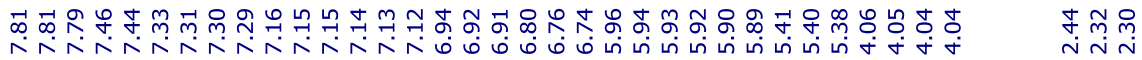

Nin<smiles>C=C=CN([As])C/C=C/c1ccccc1C</smiles>

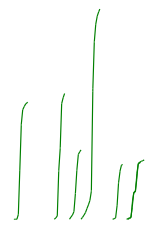

$3 b$

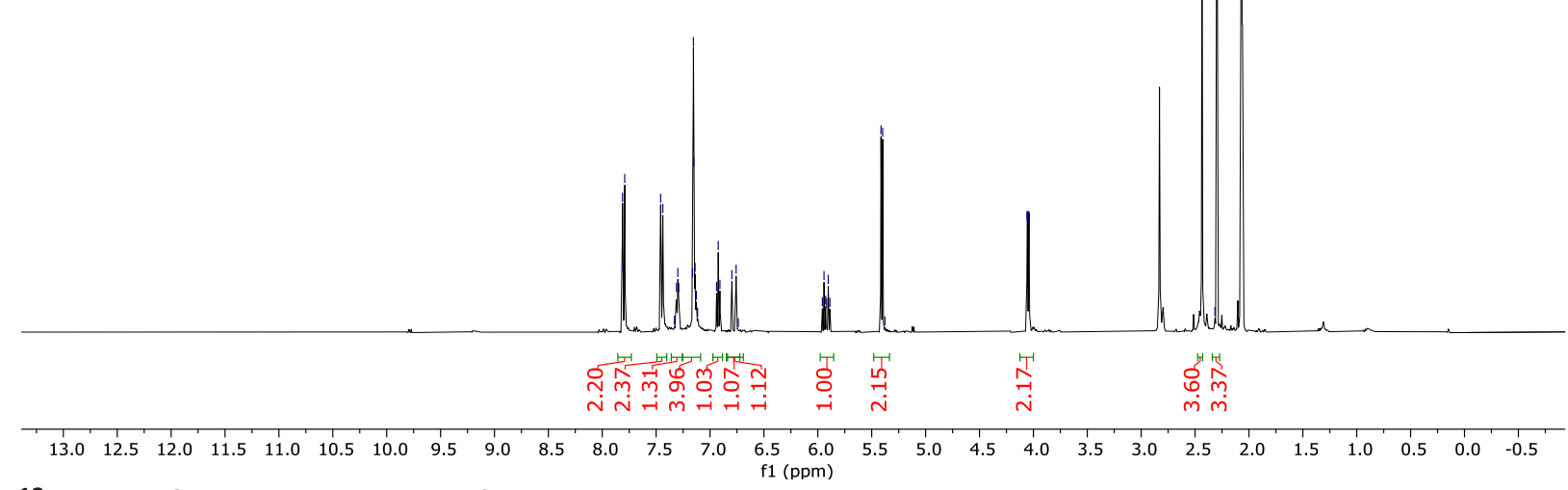

${ }^{13} \mathrm{C}$ NMR (101 MHz, acetone)

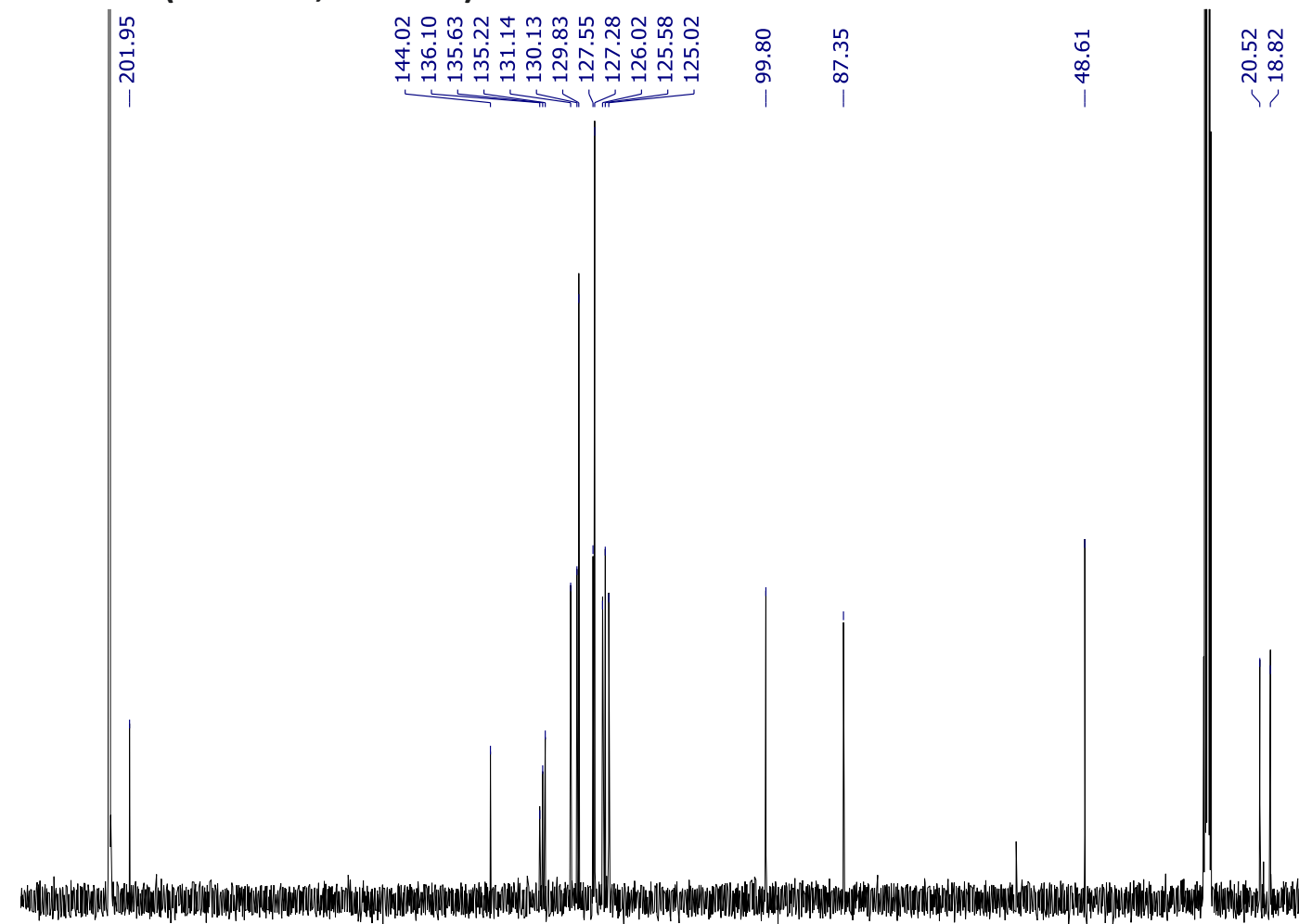


${ }^{1} \mathrm{H}$ NMR (400 MHz, acetone)

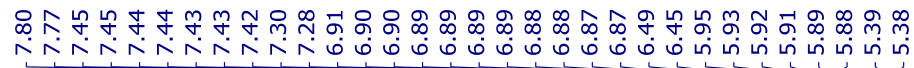

ने ने

mं mं

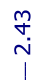<smiles>C=C=CN([As])C/C=C/c1ccc(OC)cc1</smiles>

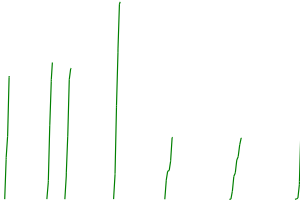

3c

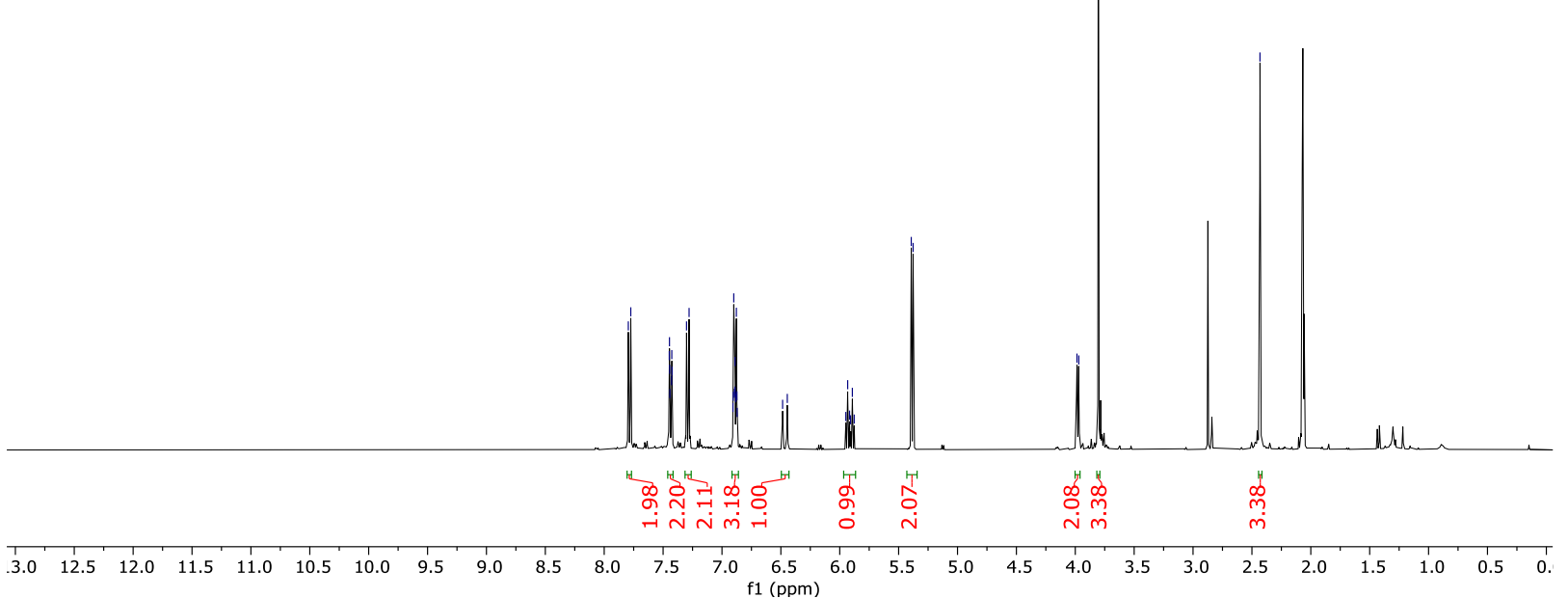

${ }^{13} \mathrm{C}$ NMR (101 MHz, acetone)

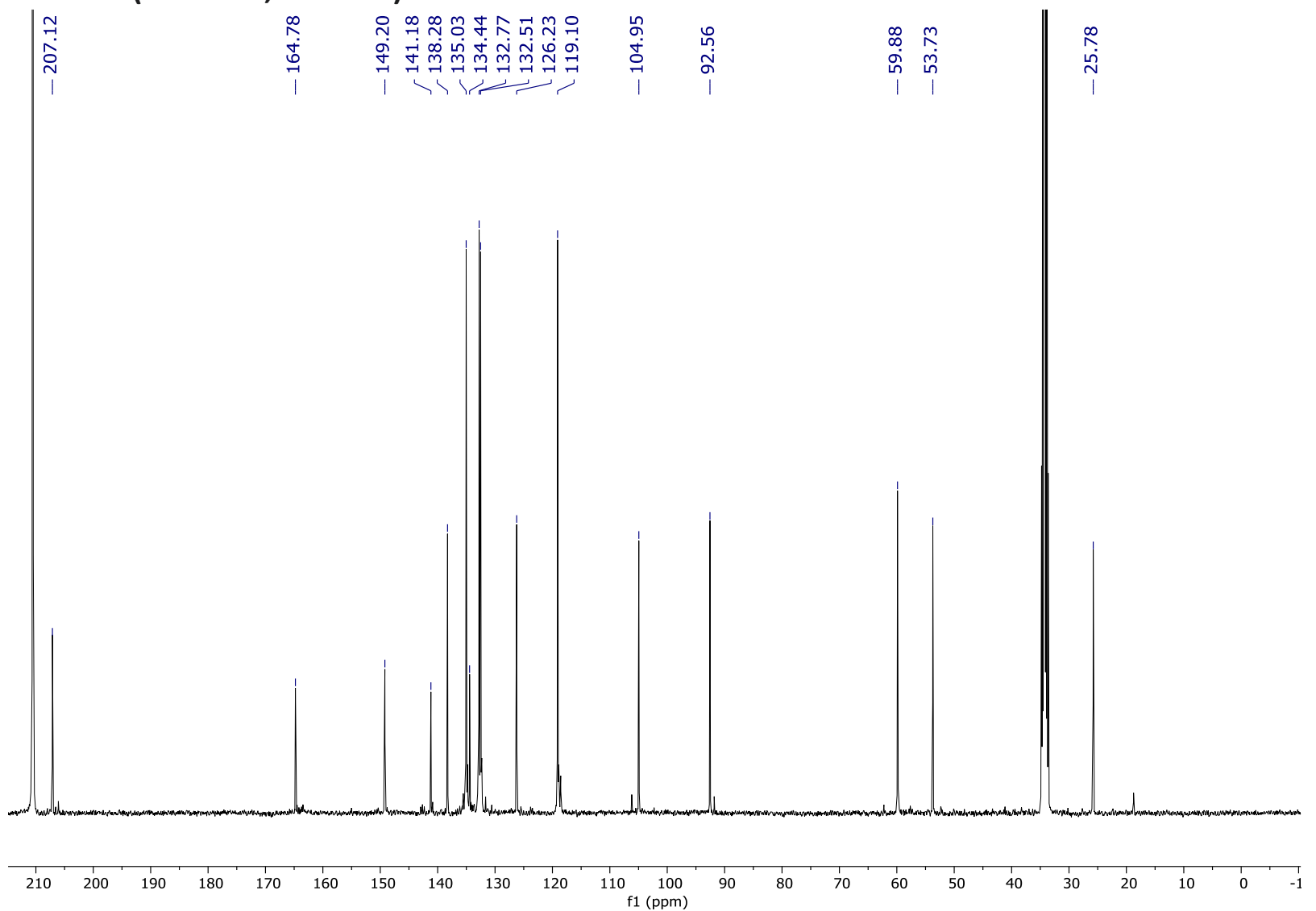


${ }^{1} \mathrm{H}$ NMR (400 MHz, $\mathrm{CDCl}_{3}$ )

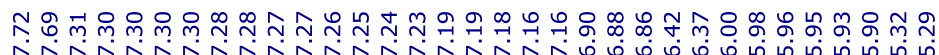

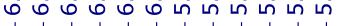

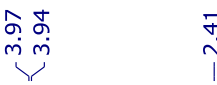<smiles>C=CCN([As])C/C=C/c1ccc(Cl)cc1</smiles>

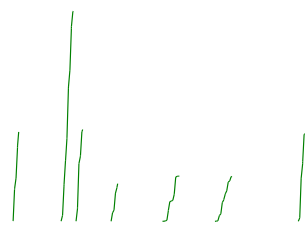

$3 d$

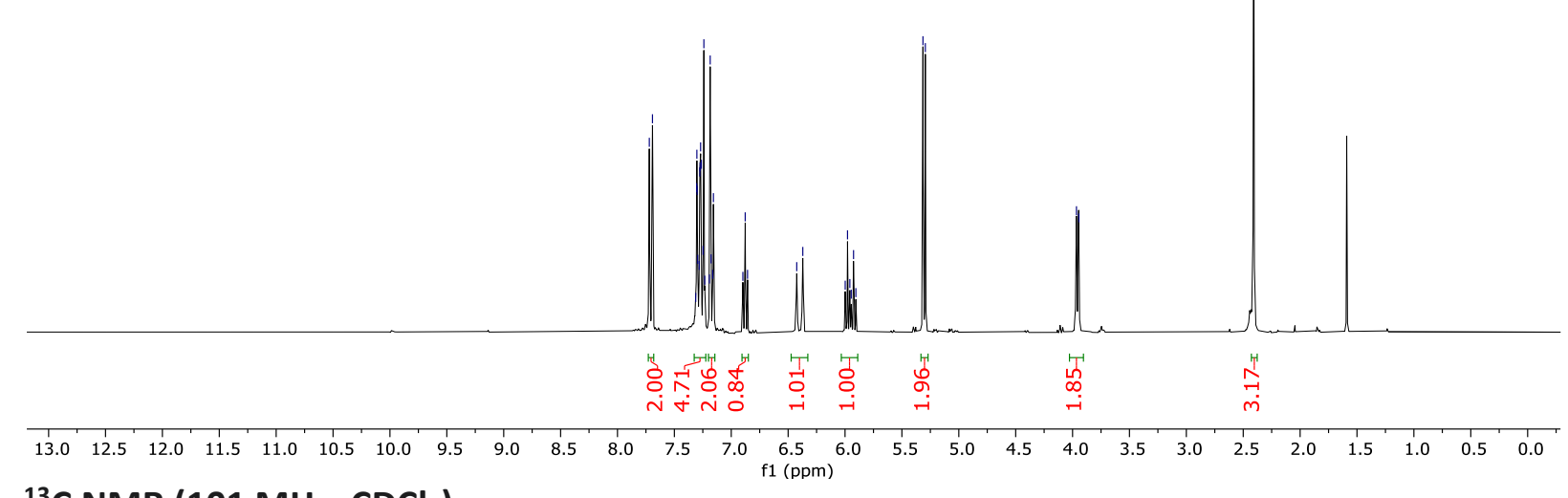

${ }^{13} \mathrm{C}$ NMR (101 MHz, $\mathrm{CDCl}_{3}$ )

ì

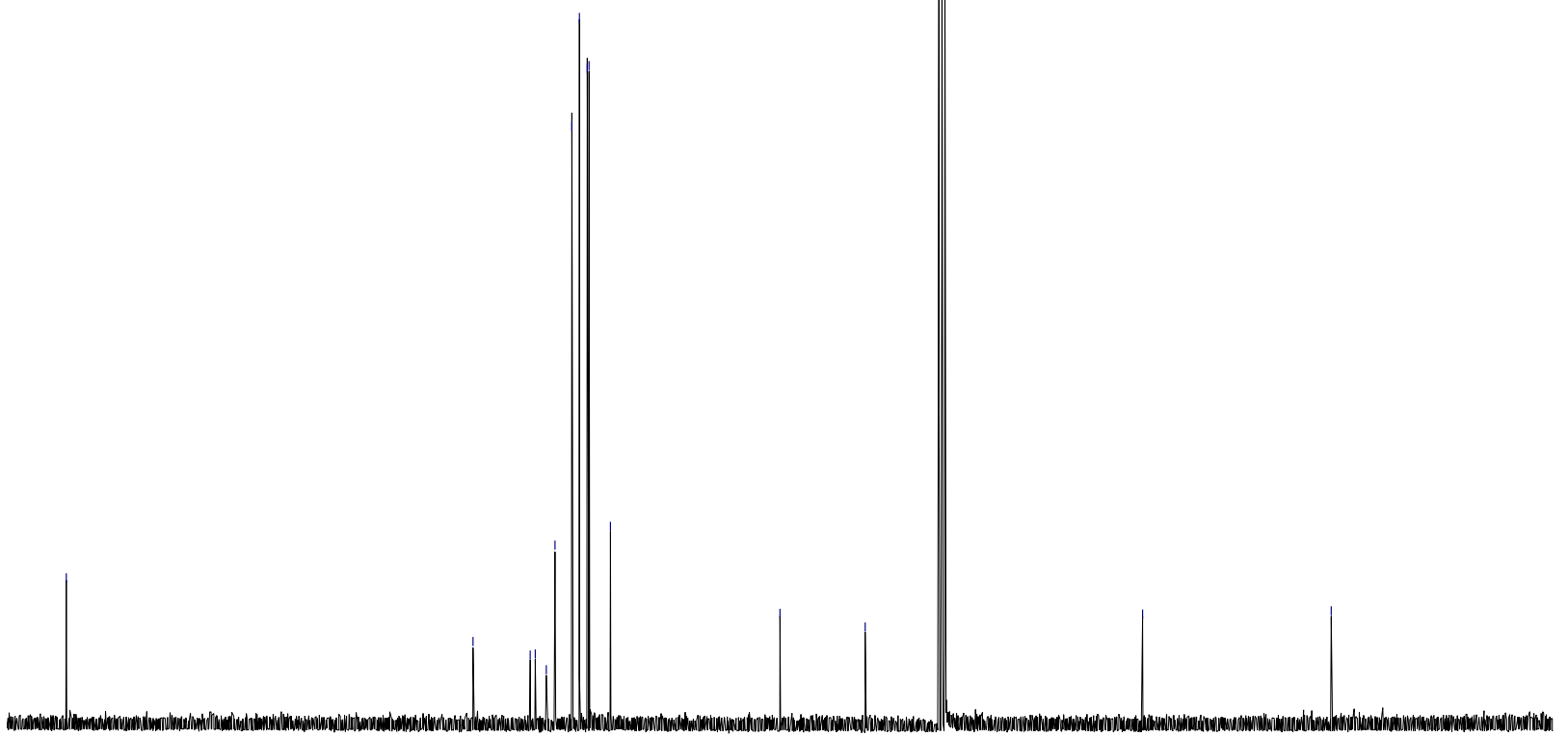

\begin{tabular}{llllllllllllllllllllllllll}
\hline 10 & 200 & 190 & 180 & 170 & 160 & 150 & 140 & 130 & 120 & 110 & 100 & 90 & 80 & 70 & 60 & 50 & 40 & 30 & 20 & 10 & 0 & -
\end{tabular} 
${ }^{1} \mathrm{H}$ NMR (400 MHz, acetone)

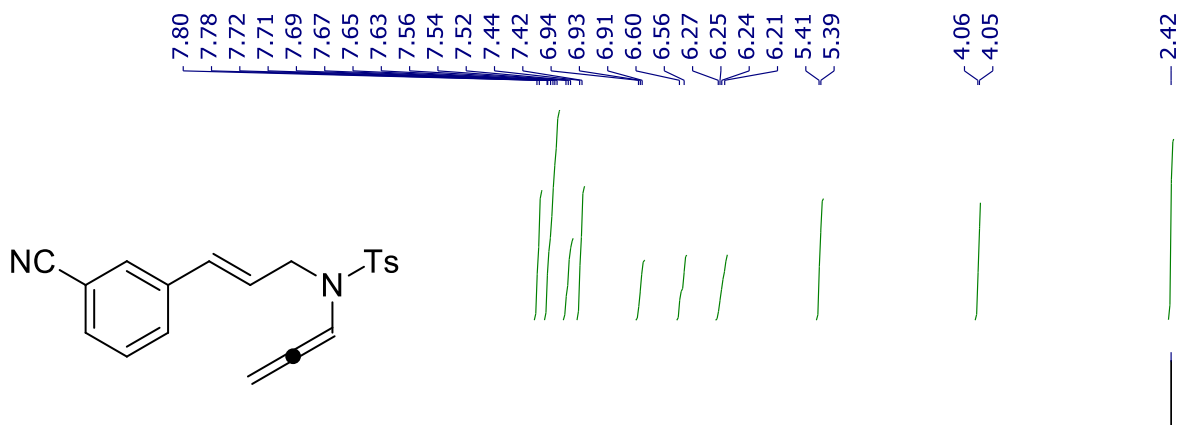

$3 e$

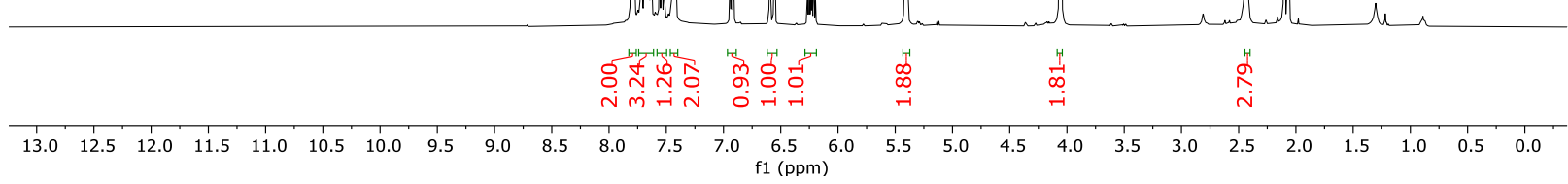

${ }^{13} \mathrm{C}$ NMR (101 MHz, acetone)

సิ

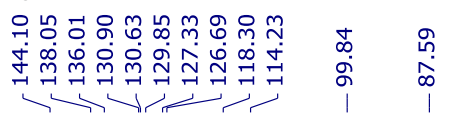

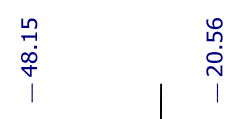

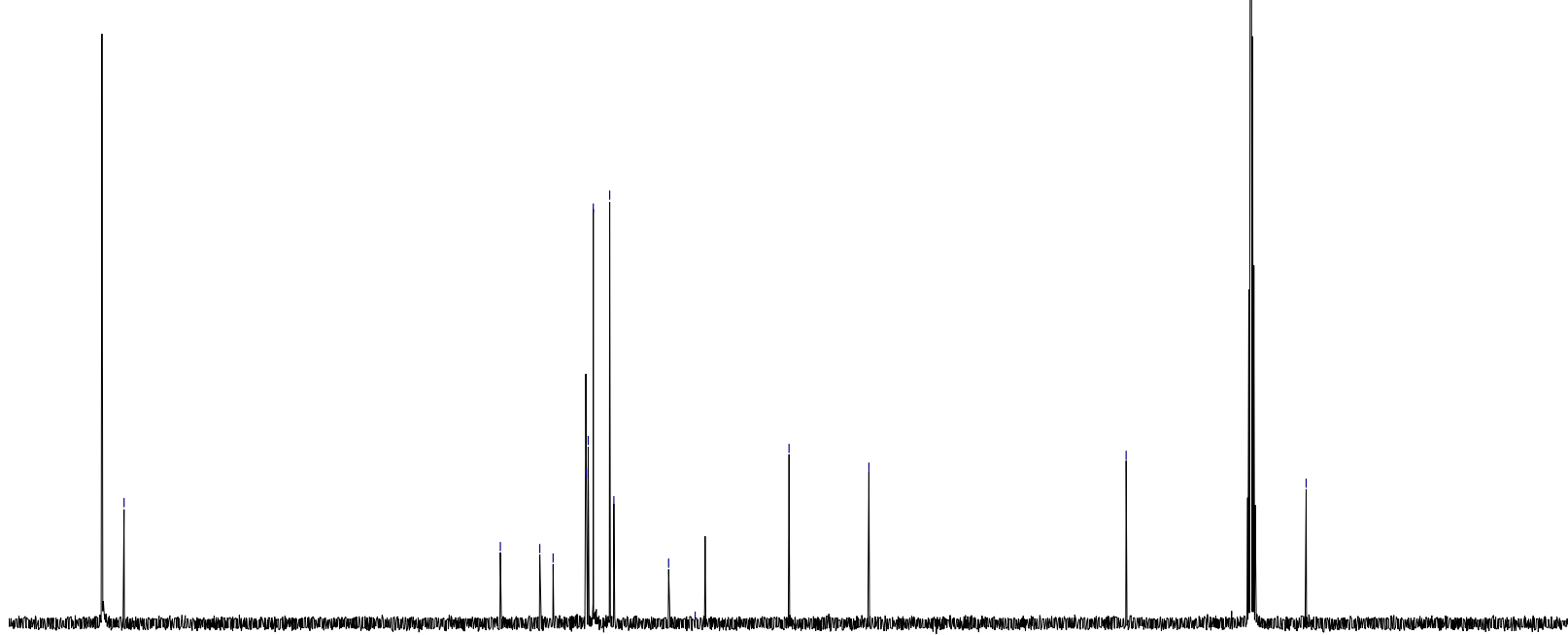

$\begin{array}{lllllllllllllllllllllll}210 & 200 & 190 & 180 & 170 & 160 & 150 & 140 & 130 & 120 & 110 & 100 & 90 & 80 & 70 & 60 & 50 & 40 & 30 & 20 & 10 & 0 & -10\end{array}$ 
${ }^{1} \mathrm{H}$ NMR $\left(400 \mathrm{MHz}, \mathrm{CDCl}_{3}\right)$

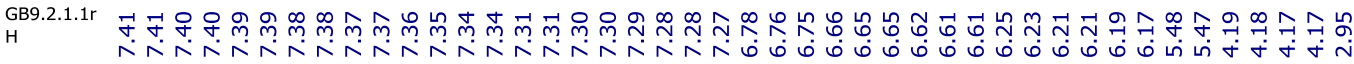<smiles>C=C=CN(C/C=C/c1ccccc1)S(C)(=O)=O</smiles>

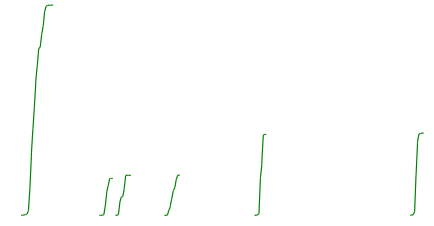

$3 f$

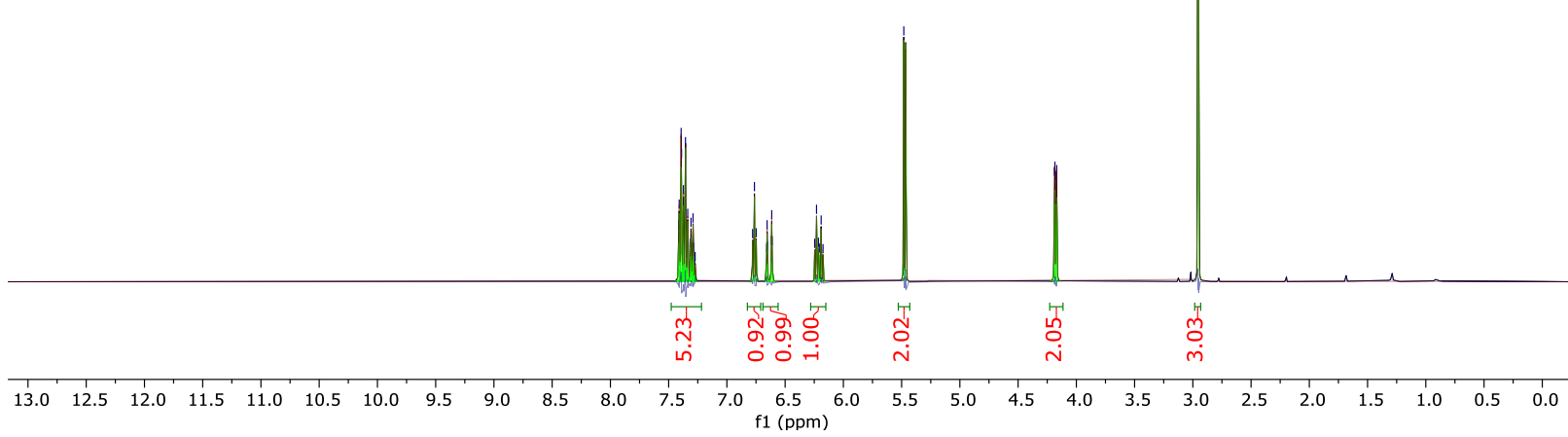

${ }^{13} \mathrm{C}$ NMR (101 MHz, $\left.\mathrm{CDCl}_{3}\right)$

GB9.3. $\stackrel{\text { Gid }}{\sim}$

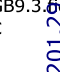

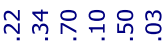

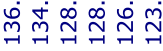

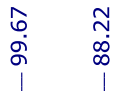

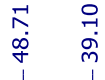

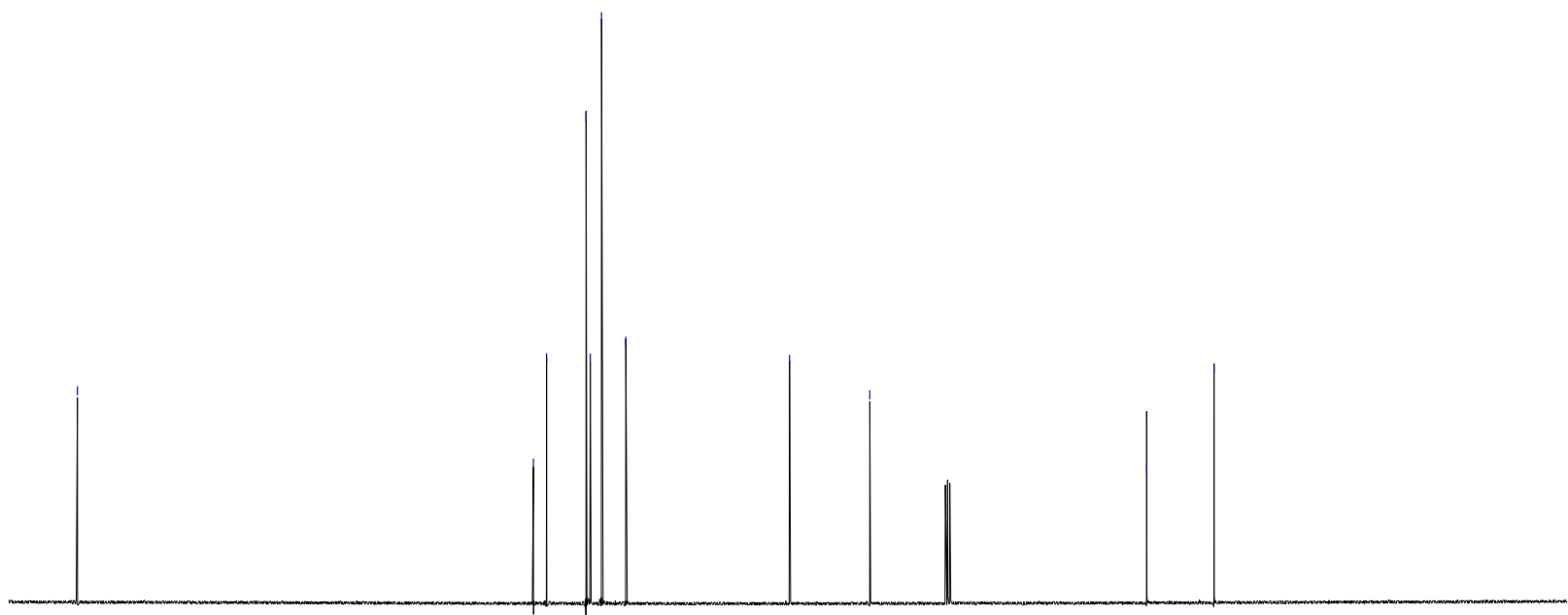

$\begin{array}{llllllllllllllllllllllll}210 & 200 & 190 & 180 & 170 & 160 & 150 & 140 & 130 & 120 & 110 & 100 & 90 & 80 & 70 & 60 & 50 & 40 & 30 & 20 & 10 & 0 & -1\end{array}$ 
${ }^{1} \mathrm{H}$ NMR (400 MHz, acetone)

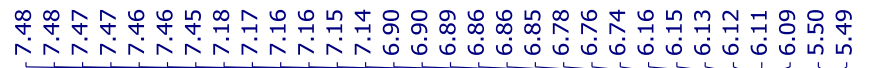<smiles>C=CCN(C/C=C/c1ccccc1C)S(C)(=O)=O</smiles>

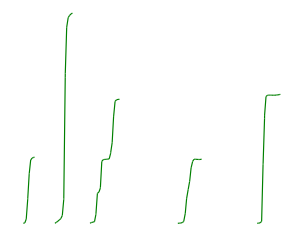

$3 g$

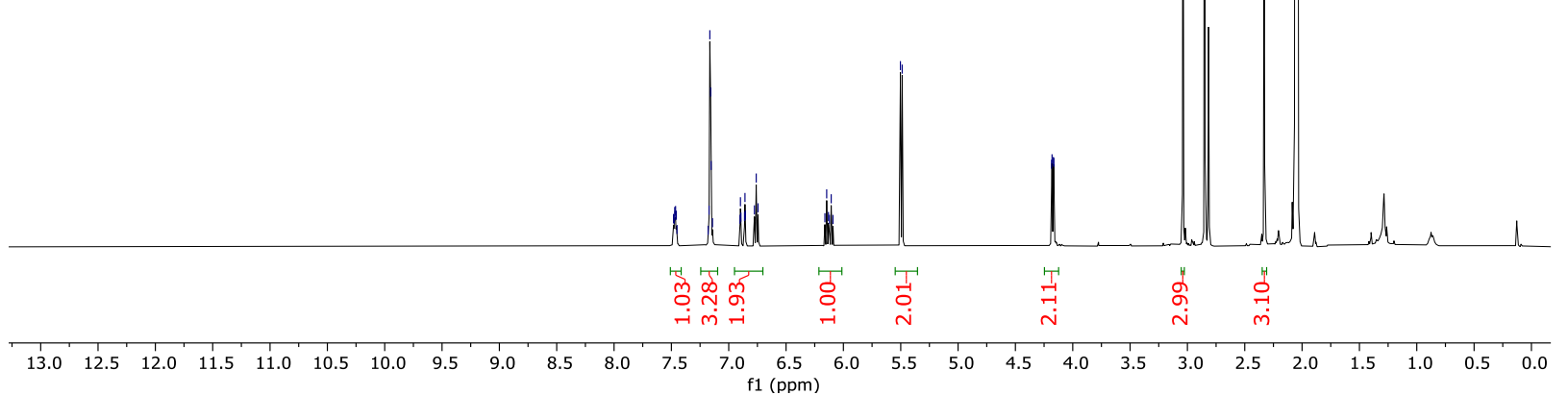

${ }^{13} \mathrm{C}$ NMR (101 MHz, acetone)

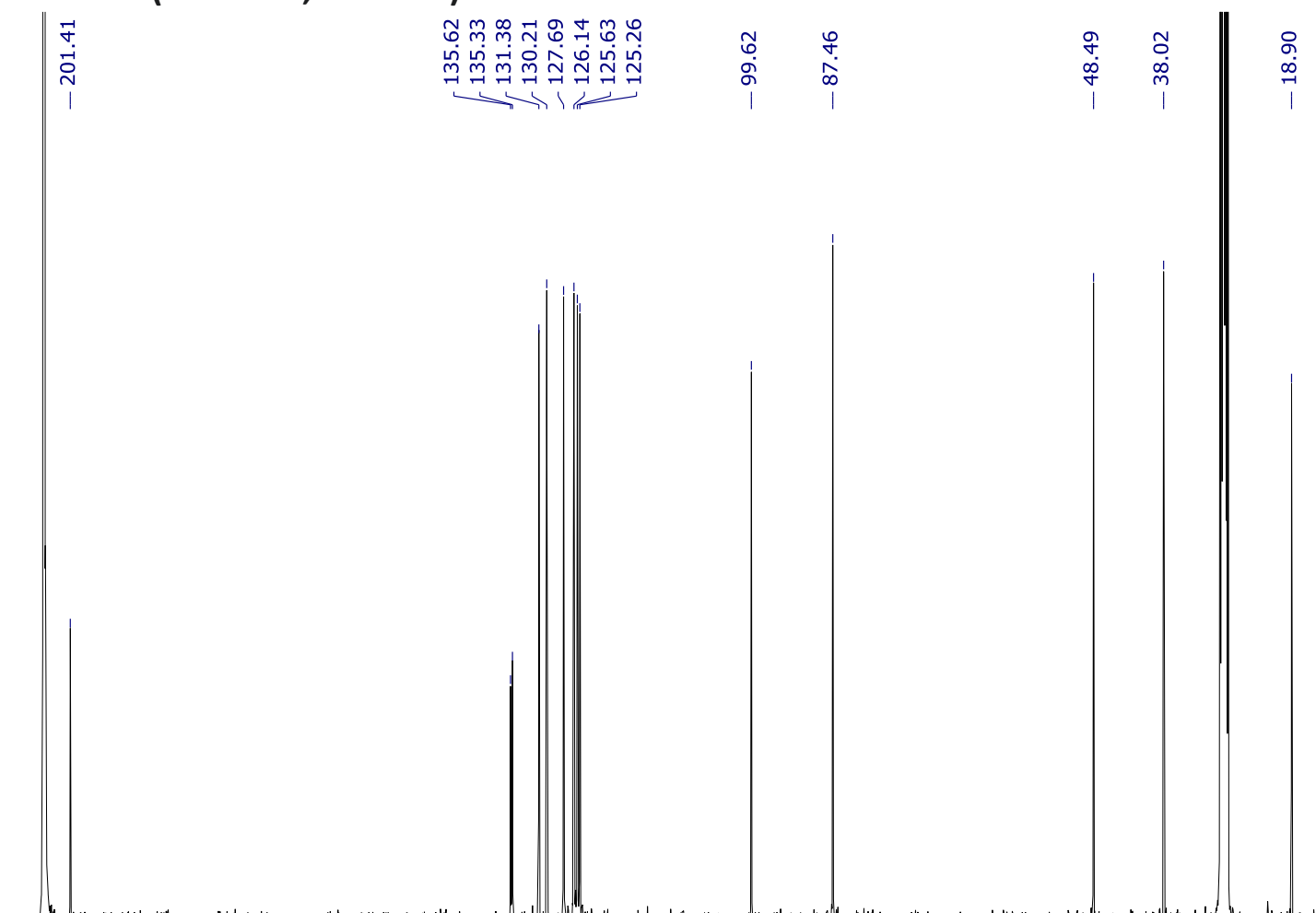

กั

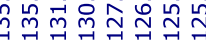

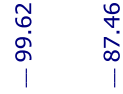

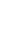


${ }^{1} \mathrm{H}$ NMR (400 MHz, acetone)

(1)

${ }^{13} \mathrm{C}$ NMR (101 MHz, acetone)

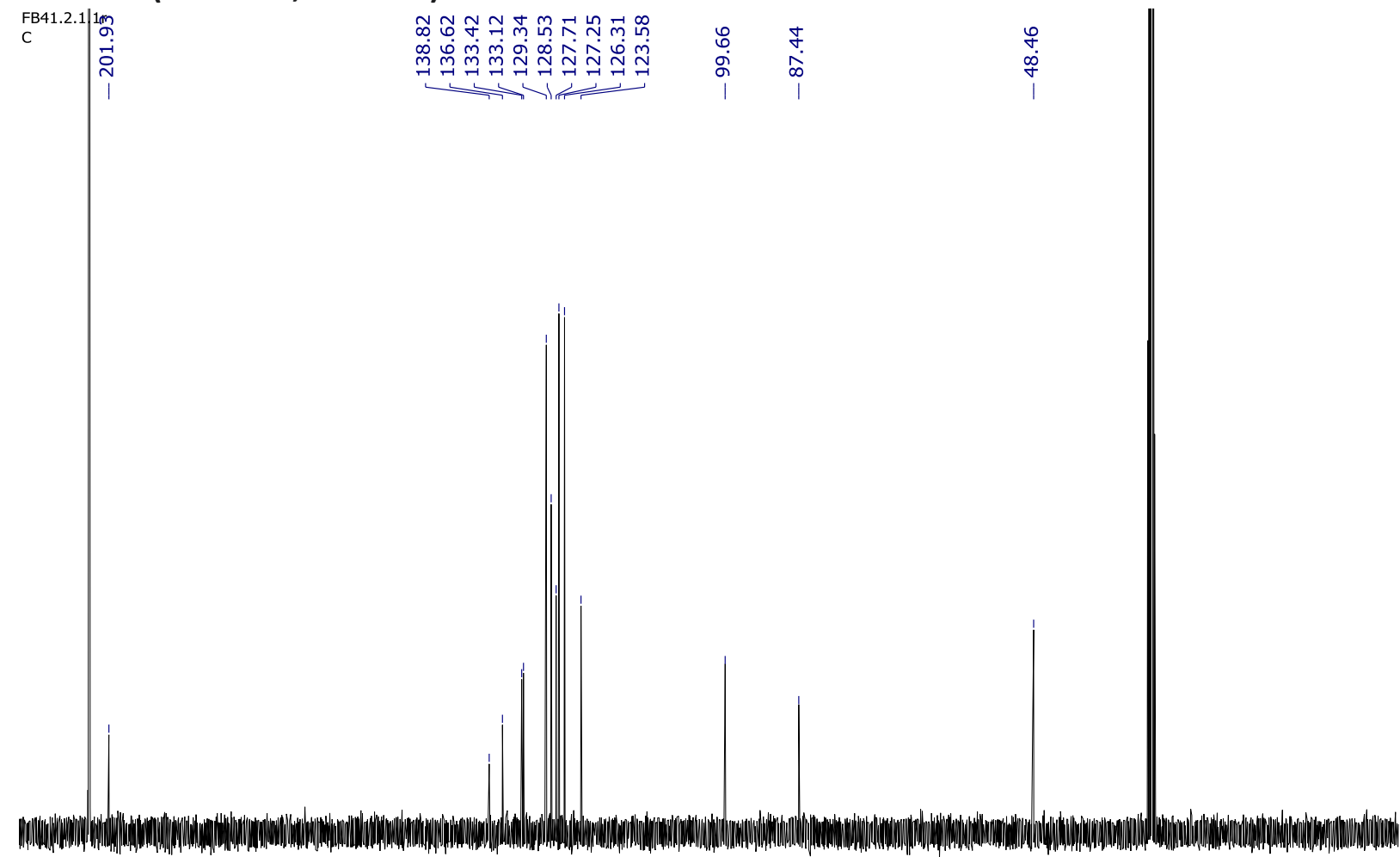

$\begin{array}{lllllllllllllllllllllllllll}210 & 200 & 190 & 180 & 170 & 160 & 150 & 140 & 130 & 120 & 110 & 100 & 90 & 80 & 70 & 60 & 50 & 40 & 30 & 20 & 10 & 0 & -10\end{array}$ 
${ }^{1} \mathrm{H}$ NMR (300 MHz, acetone)

FB 321

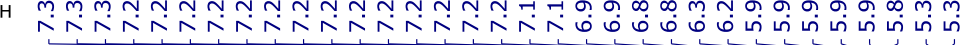

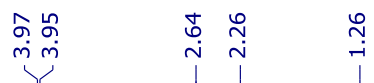<smiles>C=C=CN(C/C=C/c1ccccc1)S(=O)(=O)c1c(C)cc(C)cc1C</smiles>

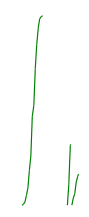

3j

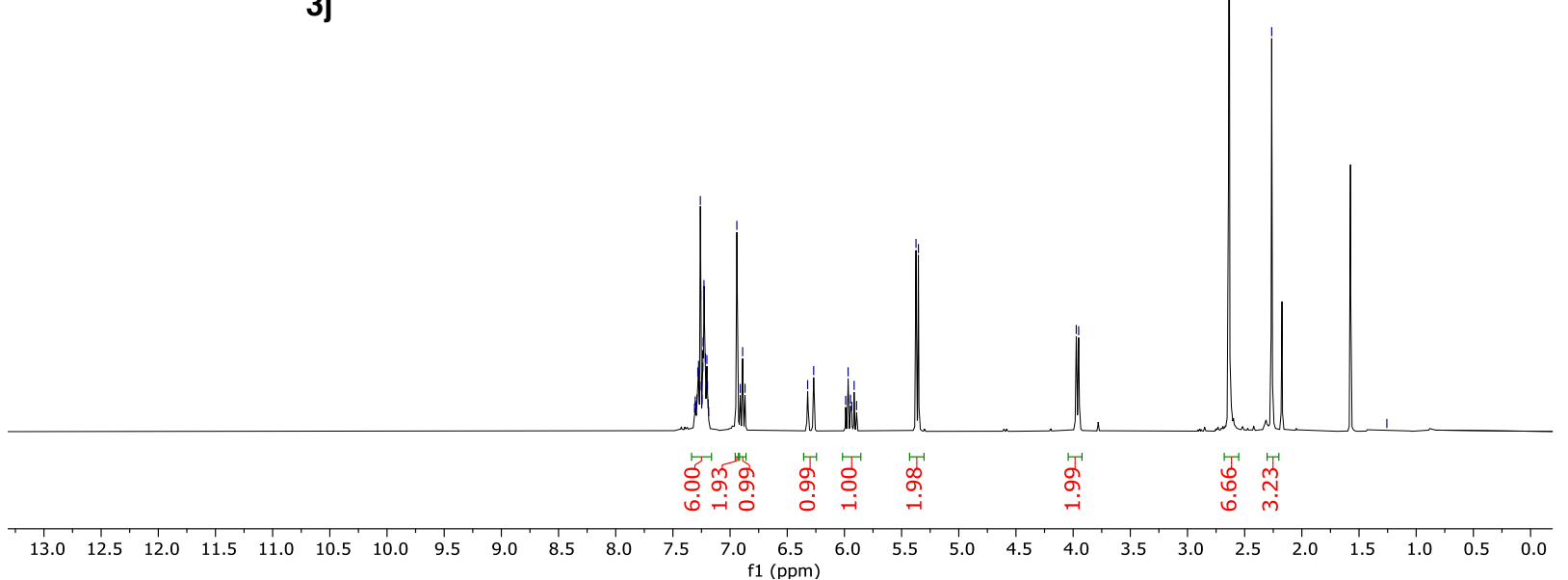

${ }^{13} \mathrm{C}$ NMR (75 MHz, acetone)

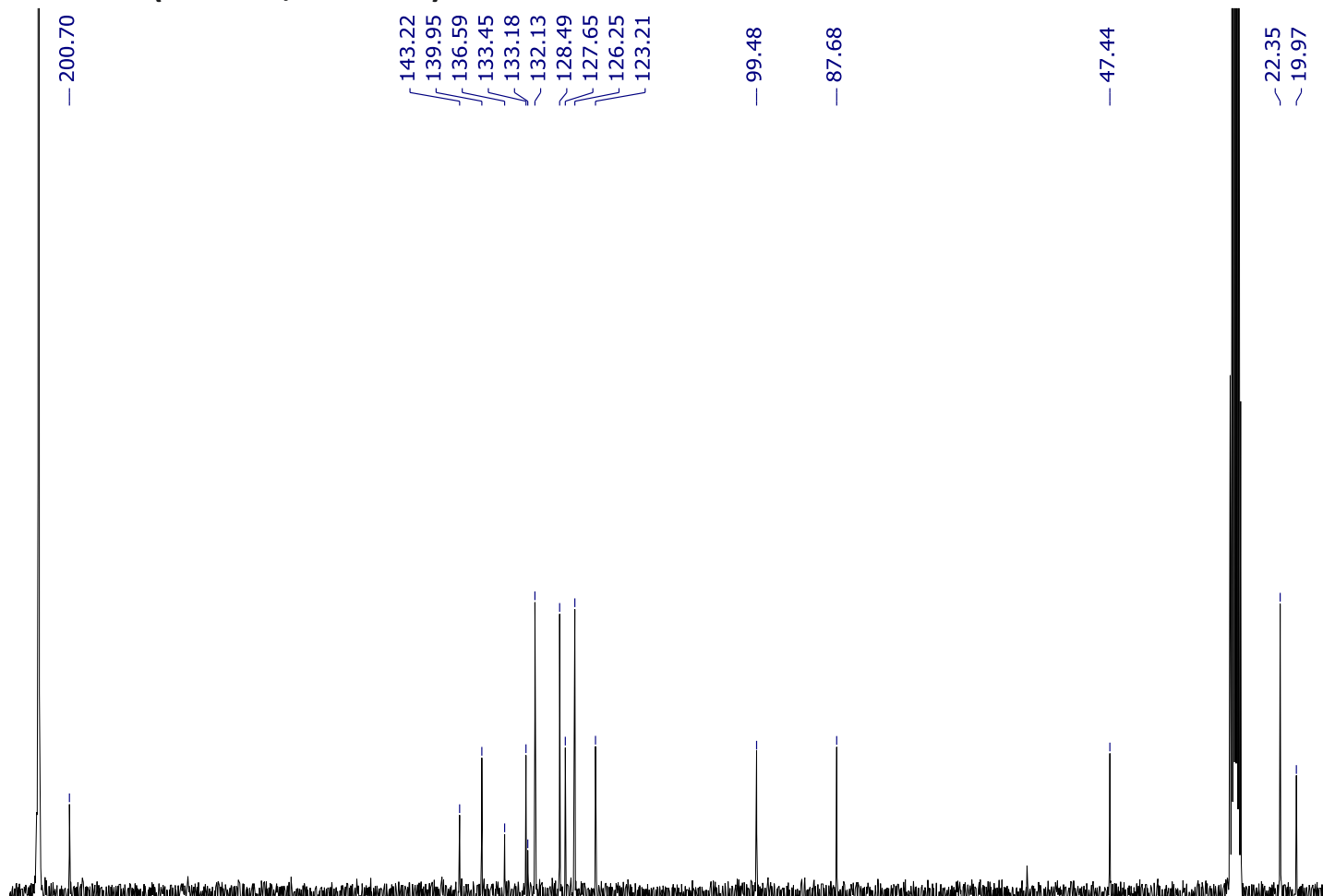

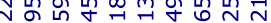

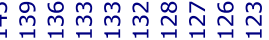


${ }^{1} \mathrm{H}$ NMR (300 MHz, $\left.\mathrm{CDCl}_{3}\right)$

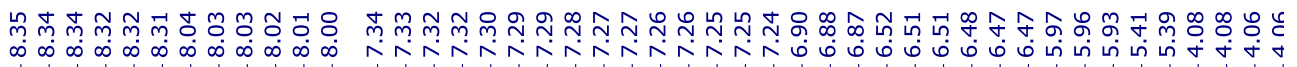

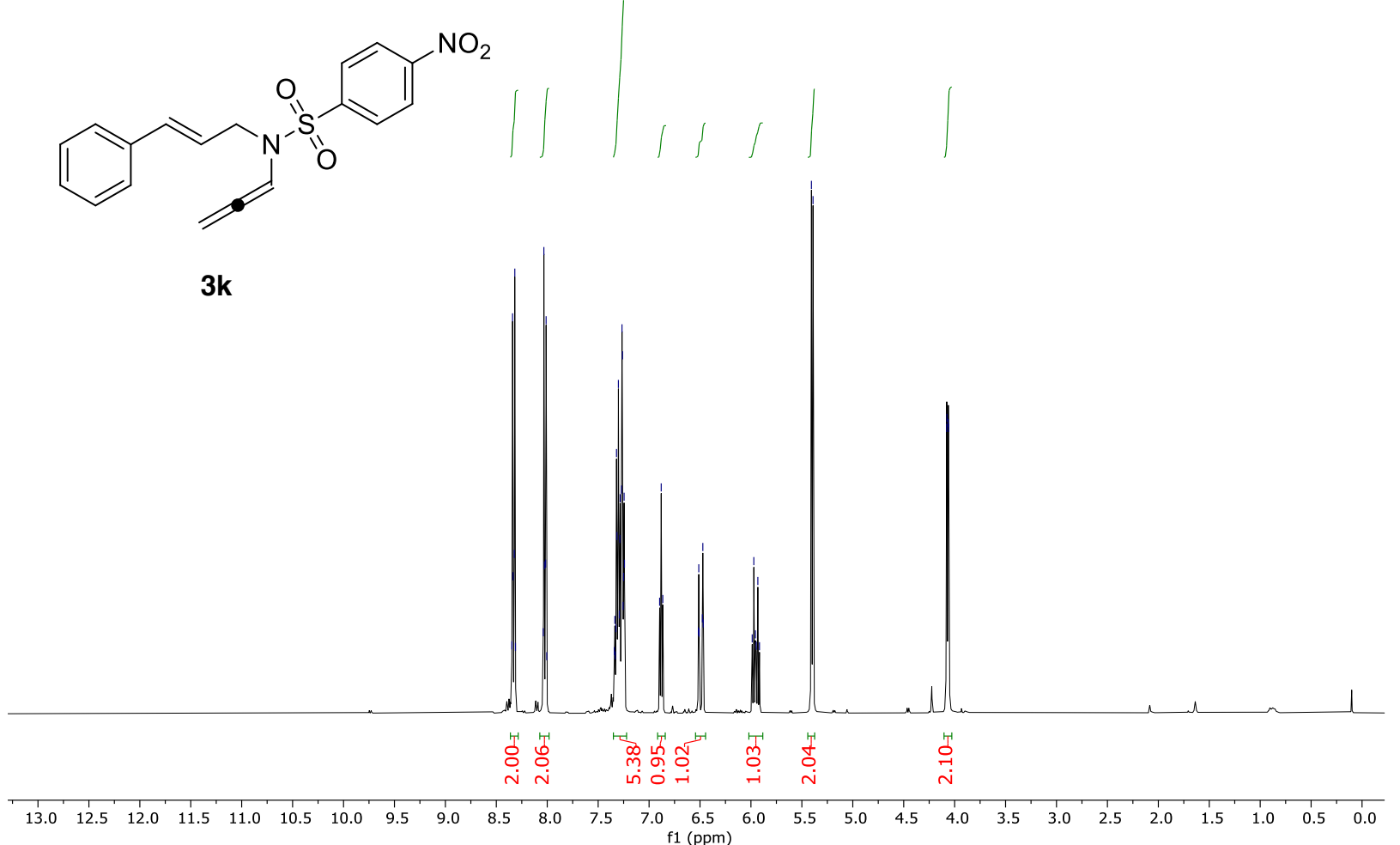

${ }^{13} \mathrm{C}$ NMR (75 MHz, $\mathrm{CDCl}_{3}$ )

品

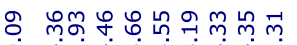

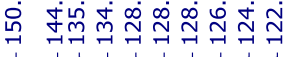

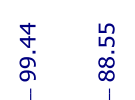

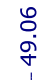

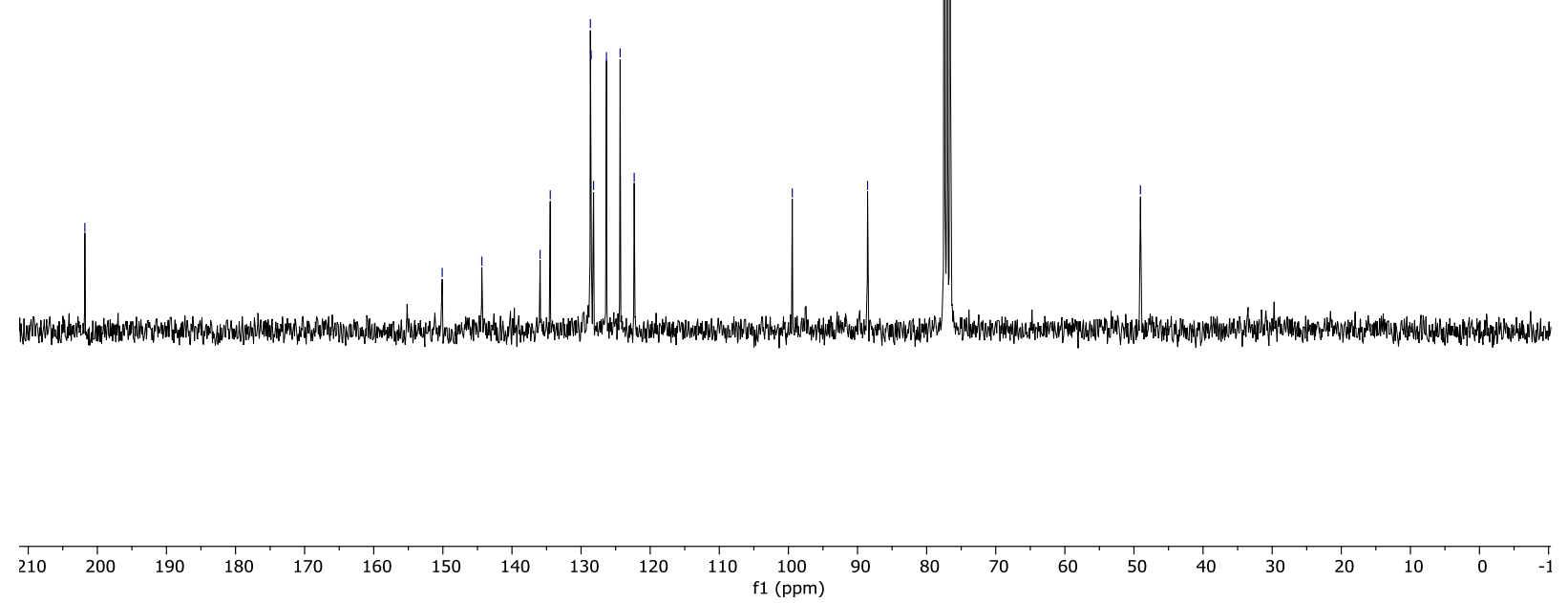




\section{${ }^{1} \mathrm{H}$ NMR (300 MHz, acetone)}

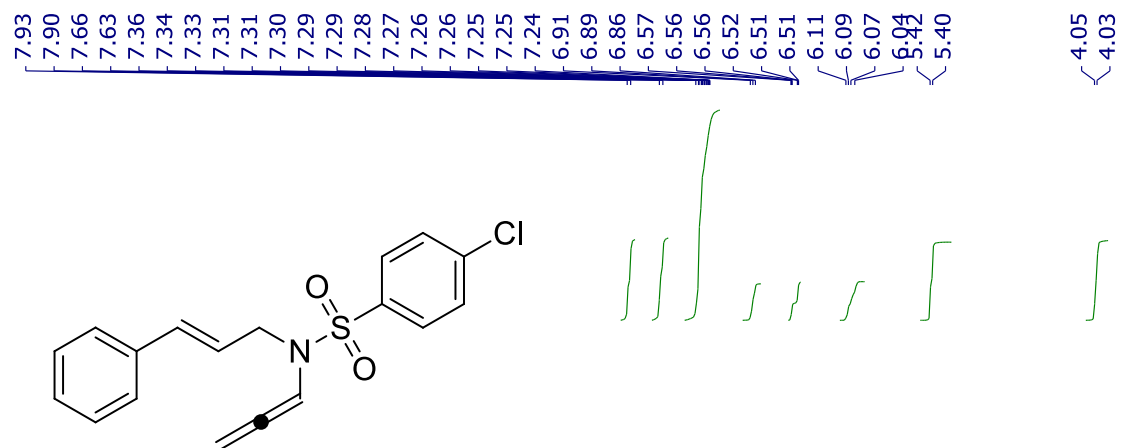

3i

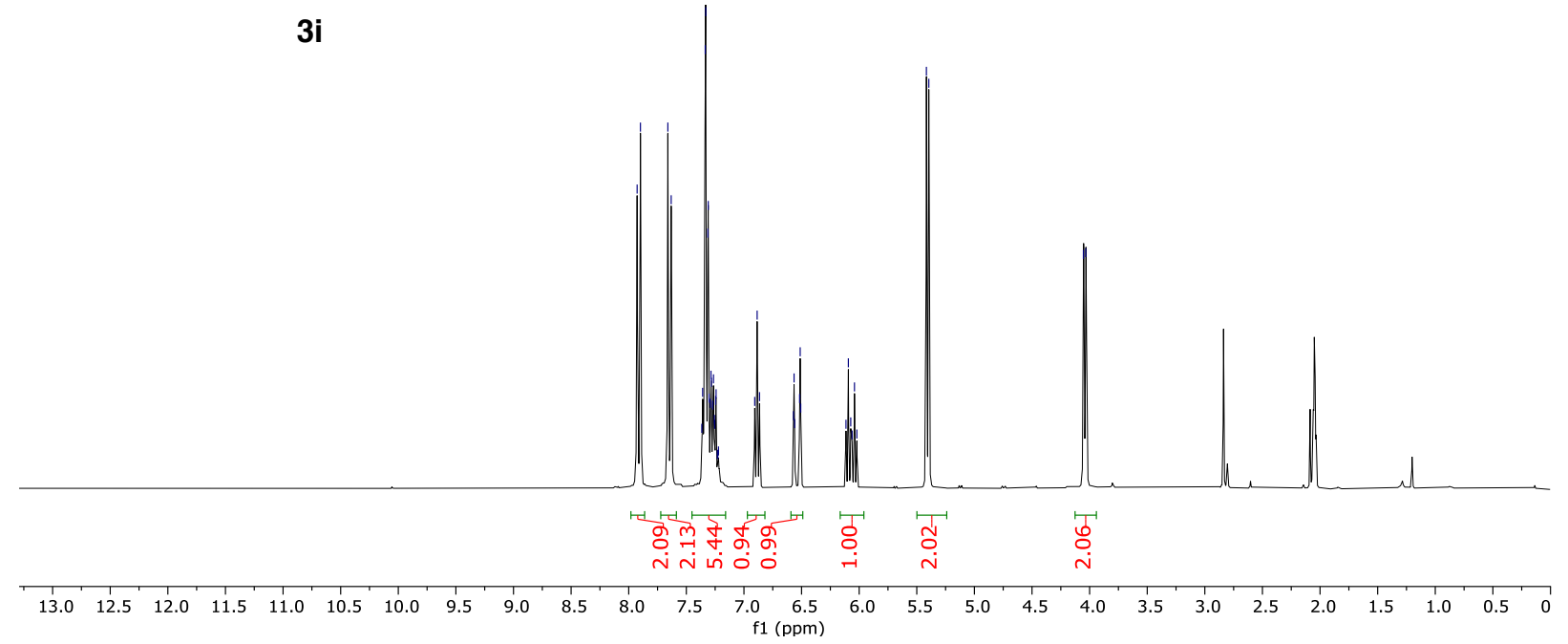

${ }^{13} \mathrm{C}$ NMR (75 MHz, acetone)

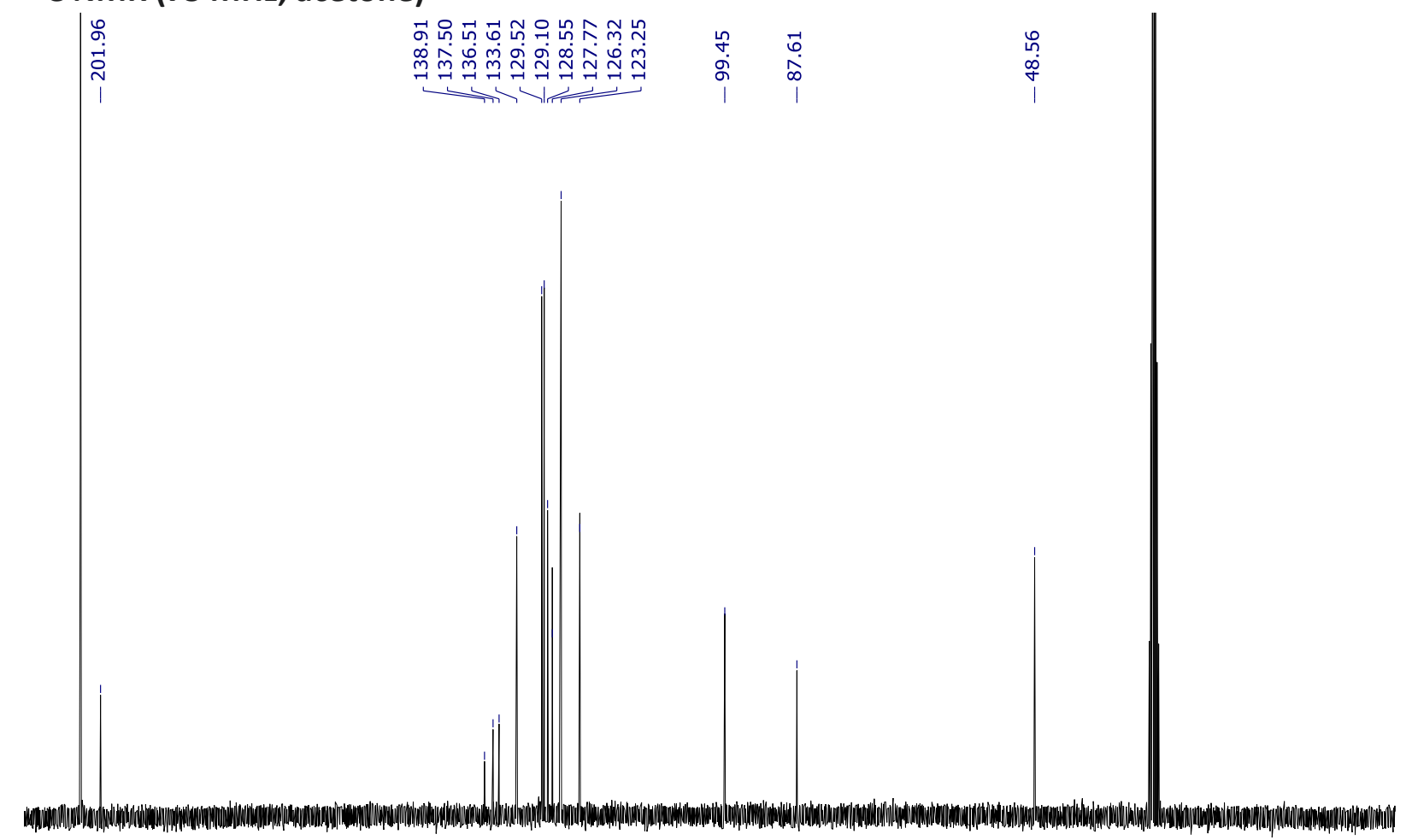

$\begin{array}{llllllllllllllllllllllllll}210 & 200 & 190 & 180 & 170 & 160 & 150 & 140 & 130 & 120 & 110 & 100 & 90 & 80 & 70 & 60 & 50 & 40 & 30 & 20 & 10 & 0 & -1\end{array}$ 
${ }^{1} \mathrm{H}$ NMR (300 MHz, acetone)

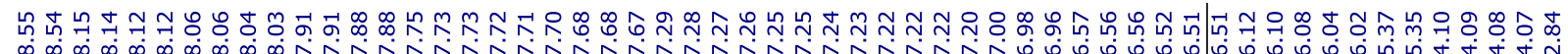
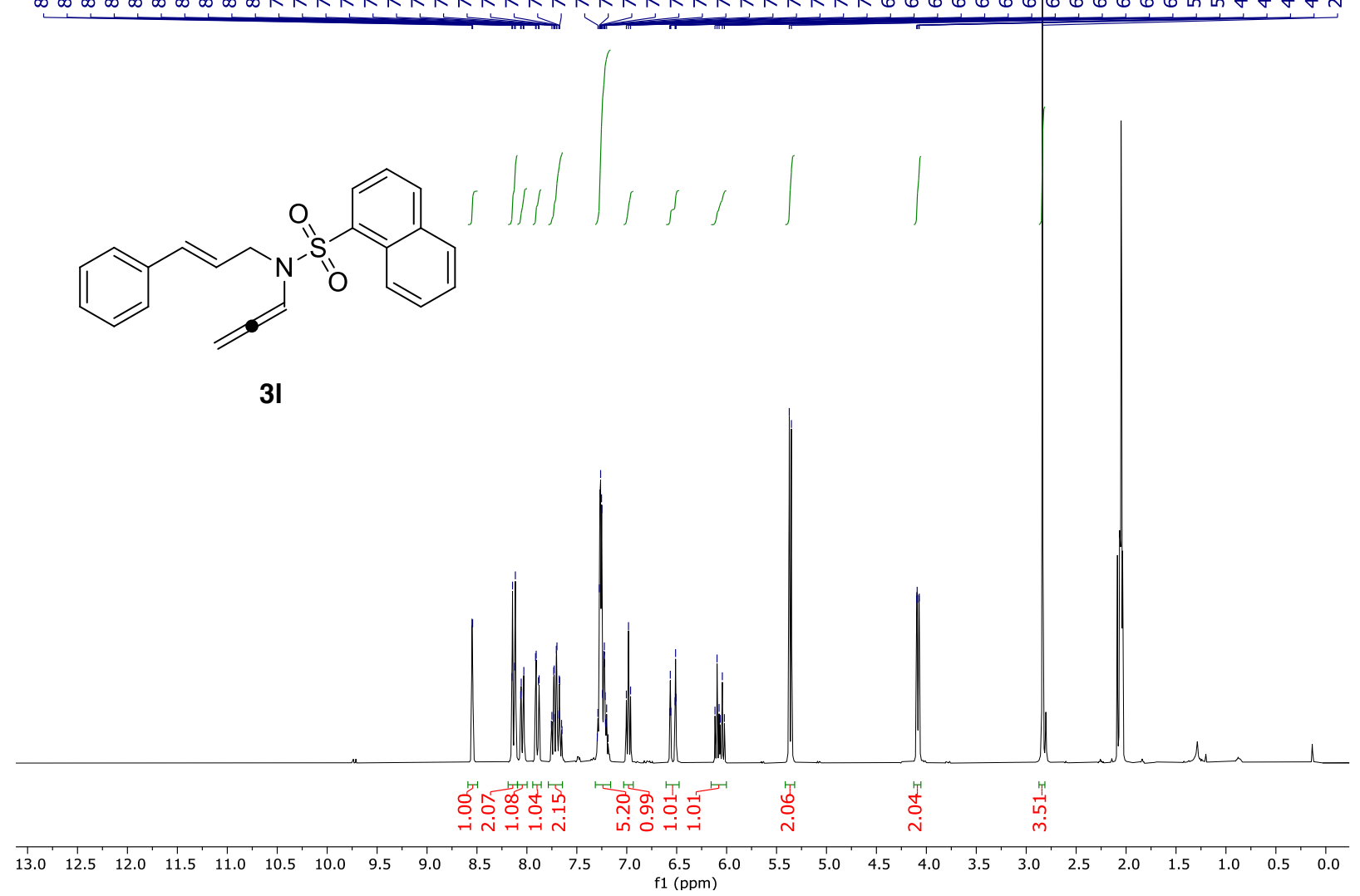

${ }^{13} \mathrm{C}$ NMR (75 MHz, acetone)

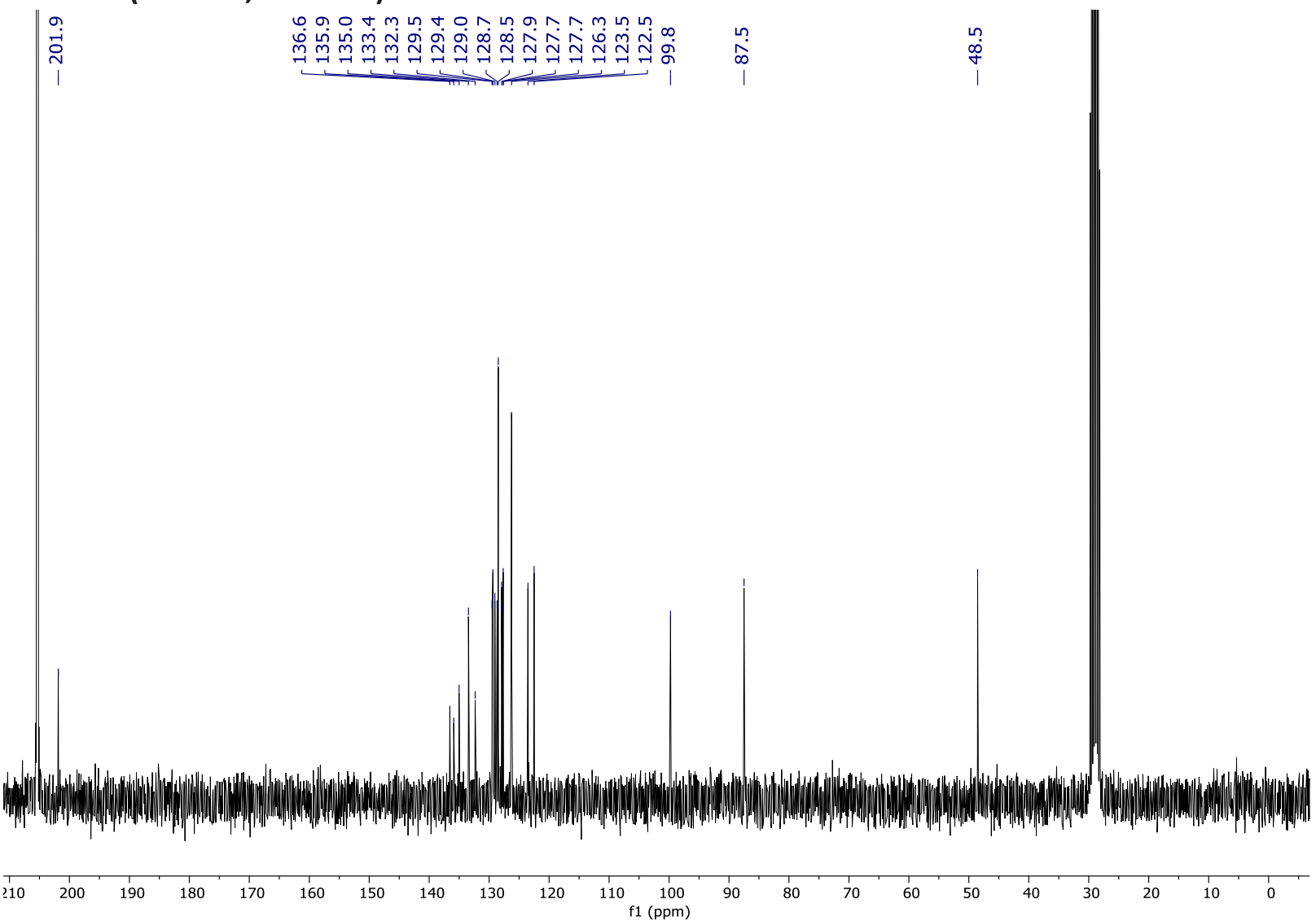


${ }^{1} \mathrm{H}$ NMR (400 MHz, acetone)

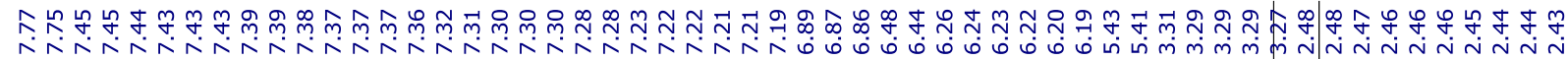<smiles>C=CN(CC/C=C/c1ccccc1)S(=O)(=O)c1ccc(C)cc1</smiles><smiles>C#CC(C)(C)C(C)C(C)(C)C=C</smiles>

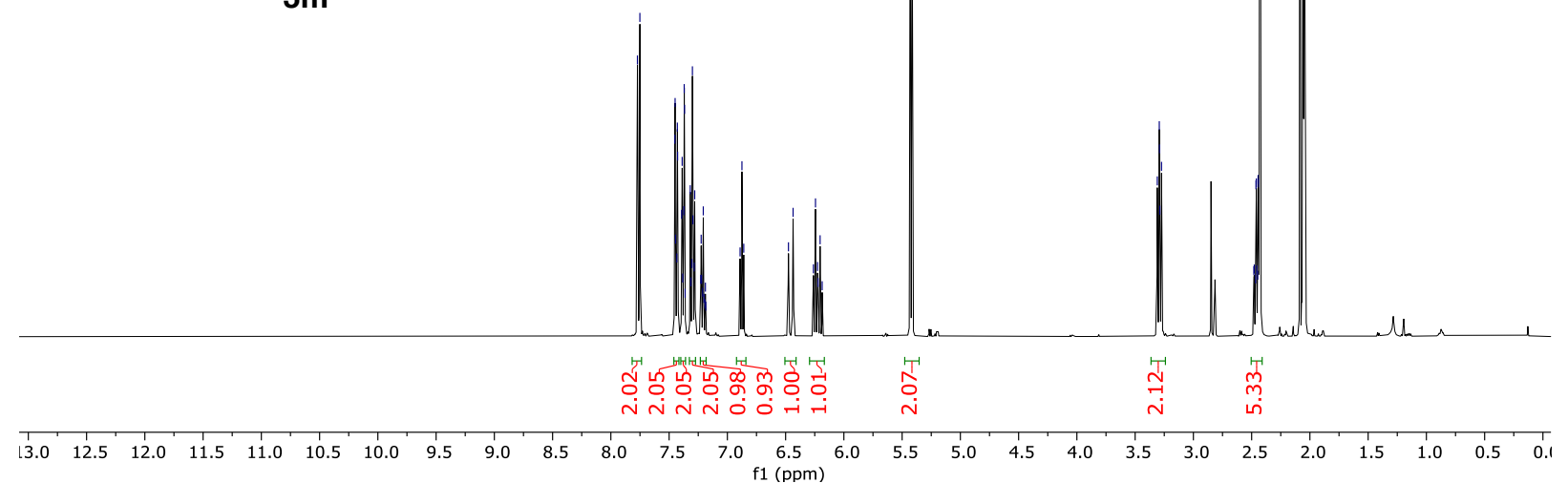

${ }^{13} \mathrm{C}$ NMR (101 MHz, acetone)

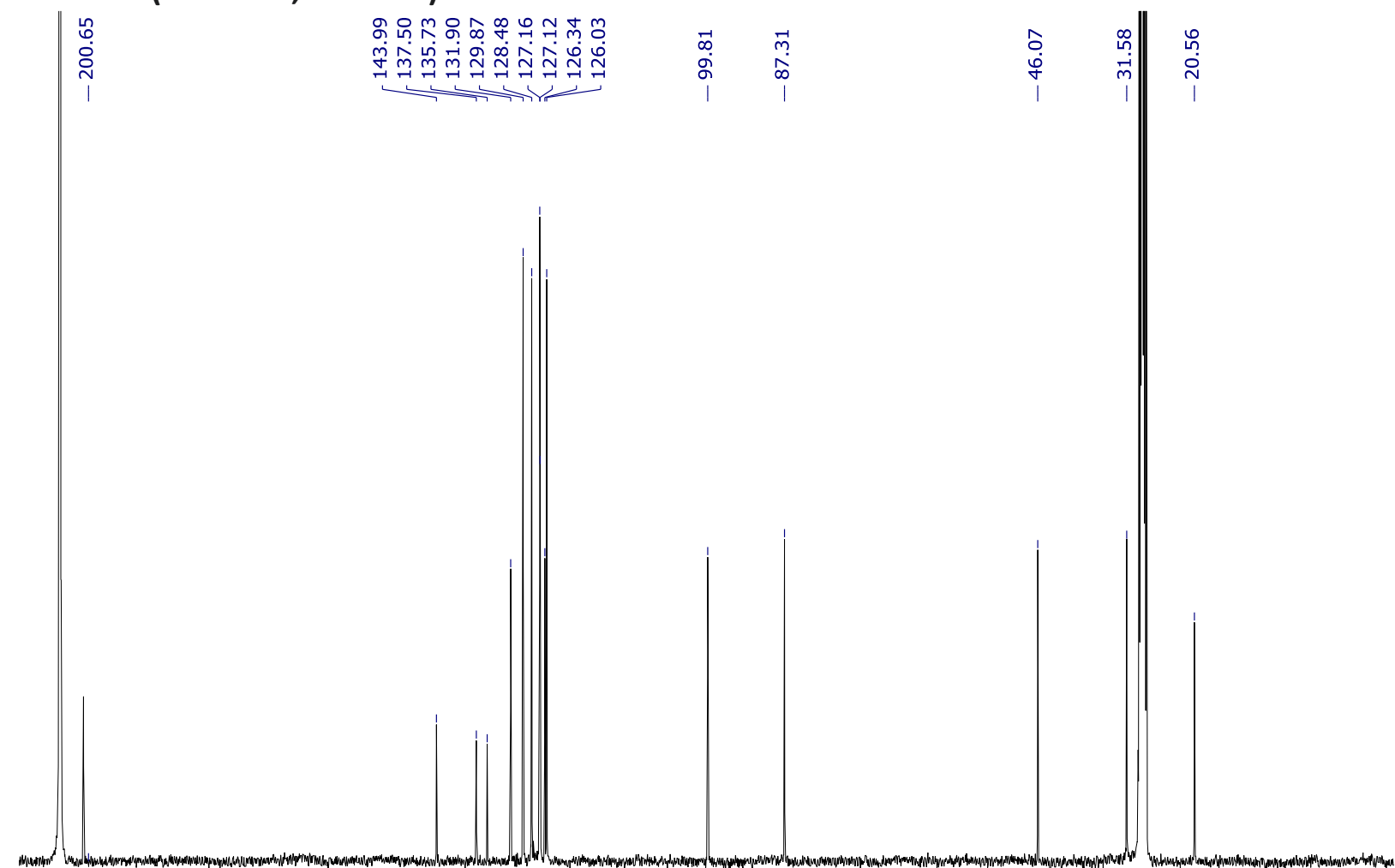

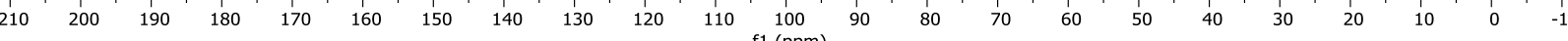


${ }^{1} \mathrm{H}$ NMR (400 MHz, $\left.\mathrm{CDCl}_{3}\right)$

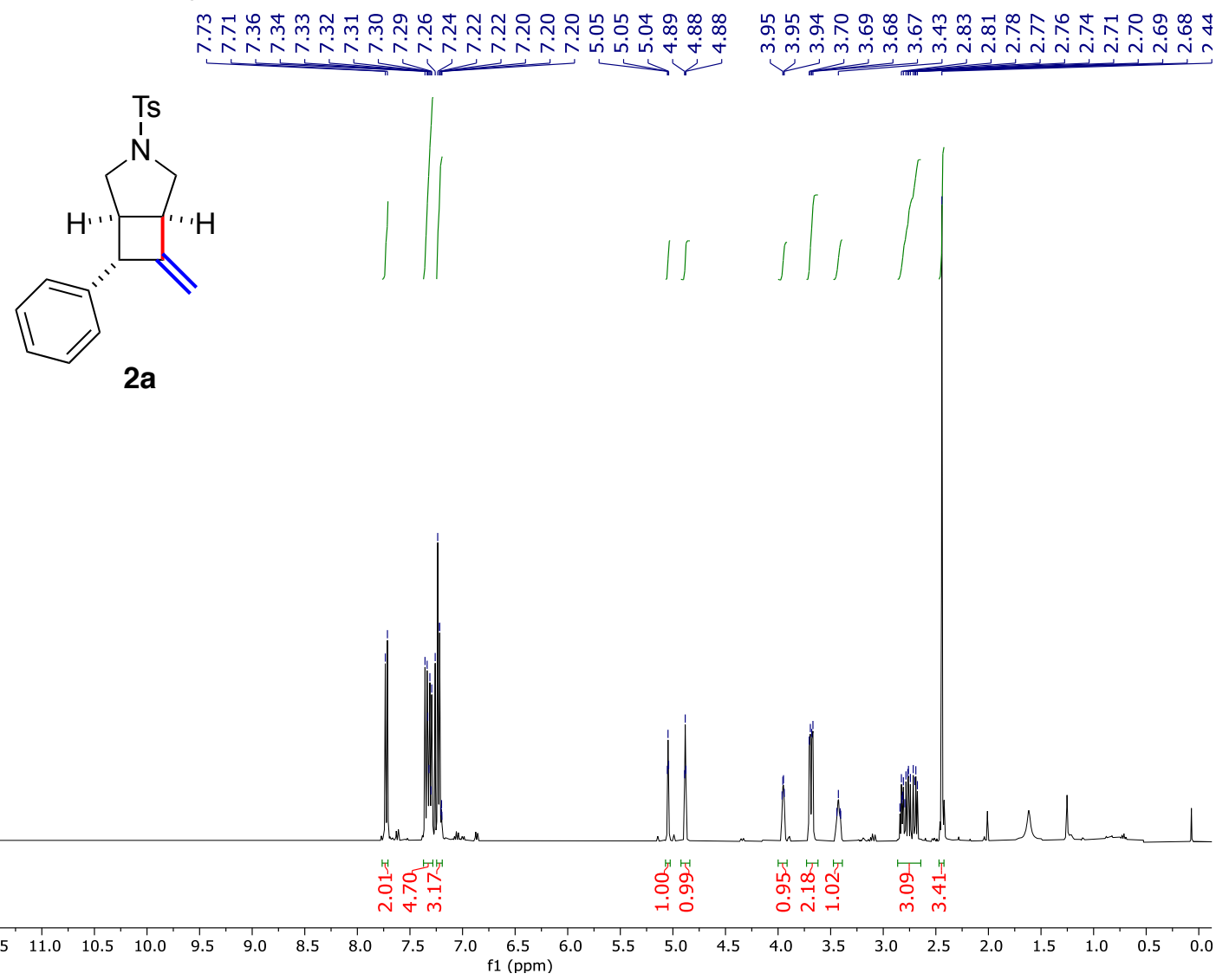

${ }^{13} \mathrm{C}$ NMR (101 $\left.\mathrm{MHz}, \mathrm{CDCl}_{3}\right)$

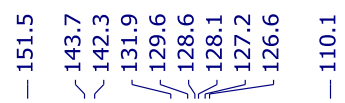

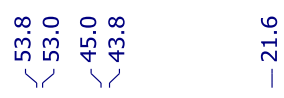

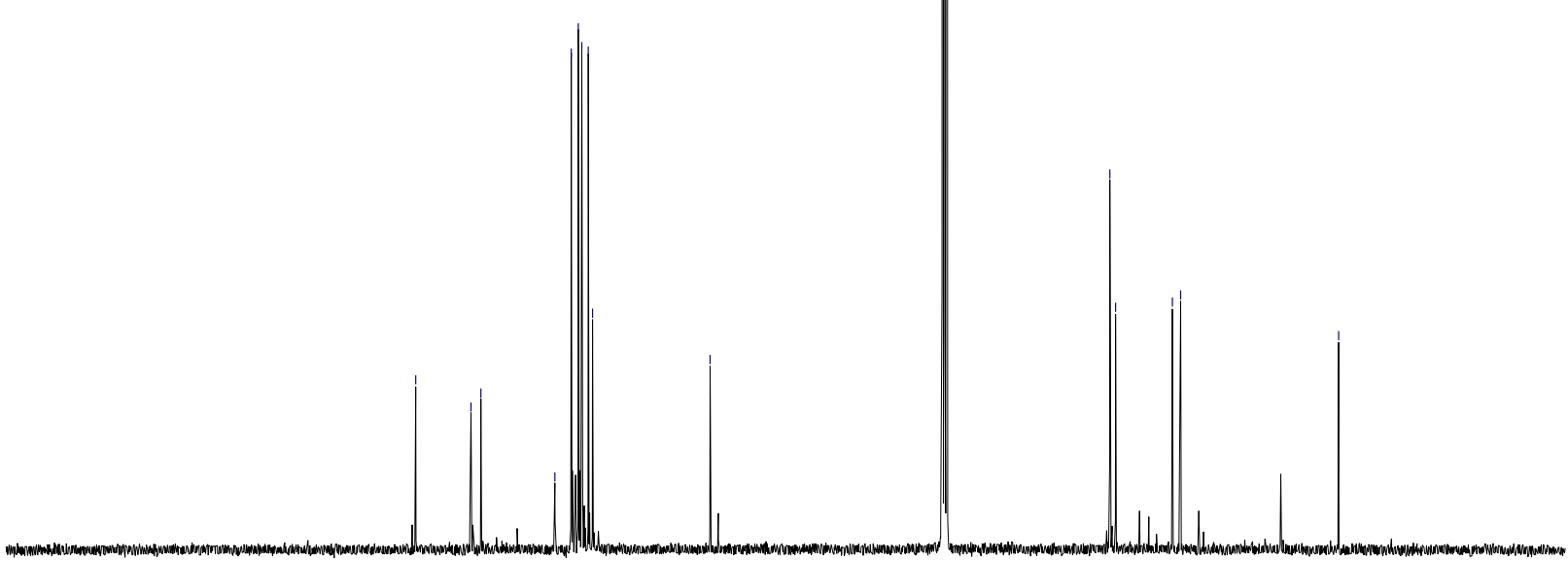

$\begin{array}{llllllllllllllllllllll}200 & 190 & 180 & 170 & 160 & 150 & 140 & 130 & 120 & 110 & 100 & 90 & 80 & 70 & 60 & 50 & 40 & 30 & 20 & 10 & 0 & -1\end{array}$ 
${ }^{1} \mathrm{H}$ NMR (300 MHz, $\mathrm{CDCl}_{3}$ )

AS175 H e C 1.1.1.1.

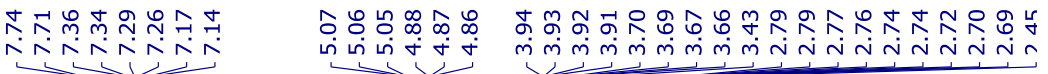<smiles>C=C1[C@H]2CN(C)C[C@H]2[C@H]1c1ccc(Cl)cc1</smiles>

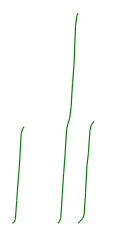

$\mathrm{Cl}^{\prime}$

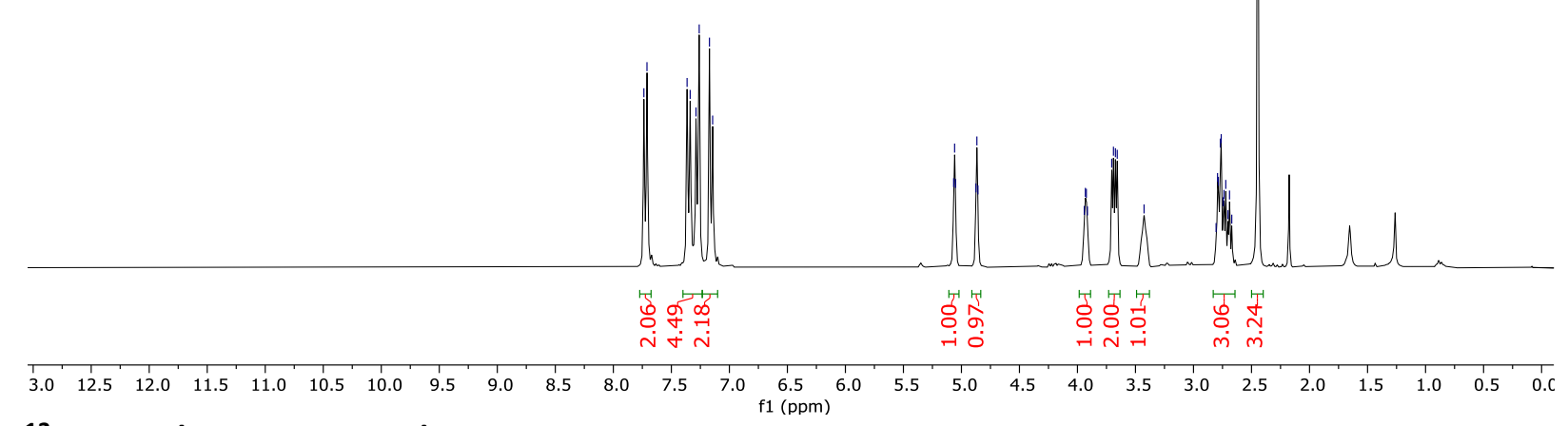

${ }^{13} \mathrm{C}$ NMR (75 MHz, $\mathrm{CDCl}_{3}$ )

AS175 H e C 1.2.1.1r

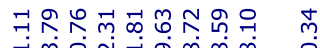

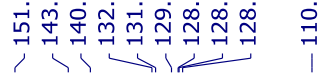

충ํㅇํำ

กึ่

セ̊ำ

in

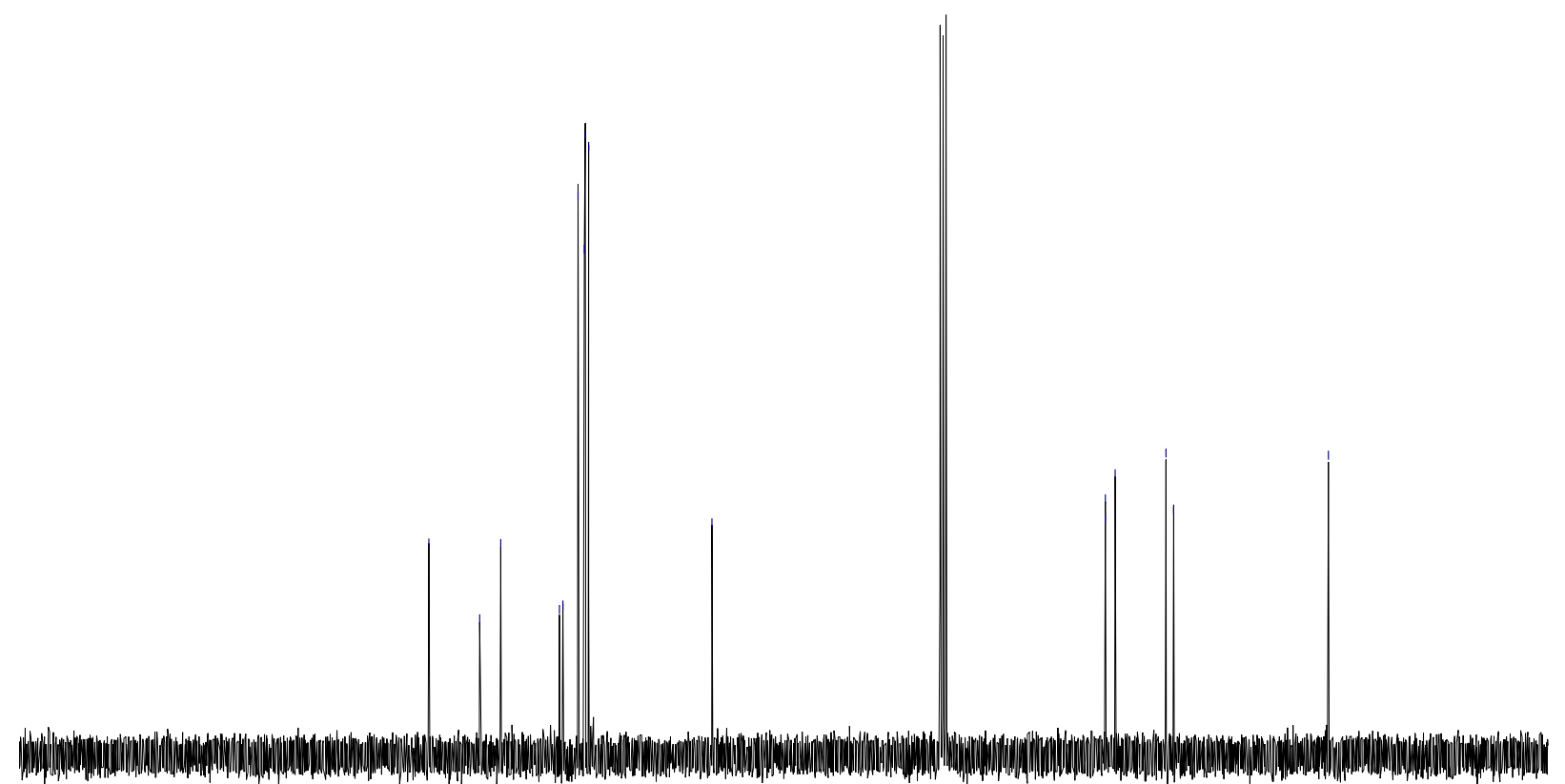

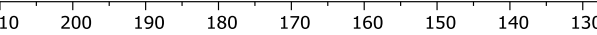
120 100
$\mathrm{f} 1(\mathrm{ppm})$ 
${ }^{1} \mathrm{H}$ NMR (400 MHz, $\mathrm{CDCl}_{3}$ )

AS180.1.1.1r

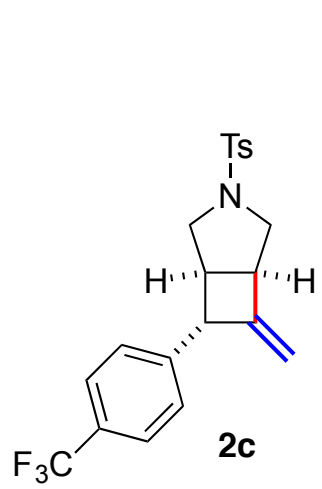

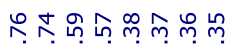

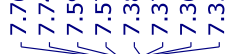

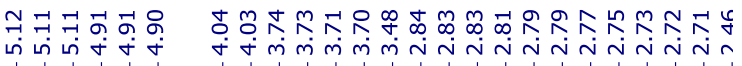

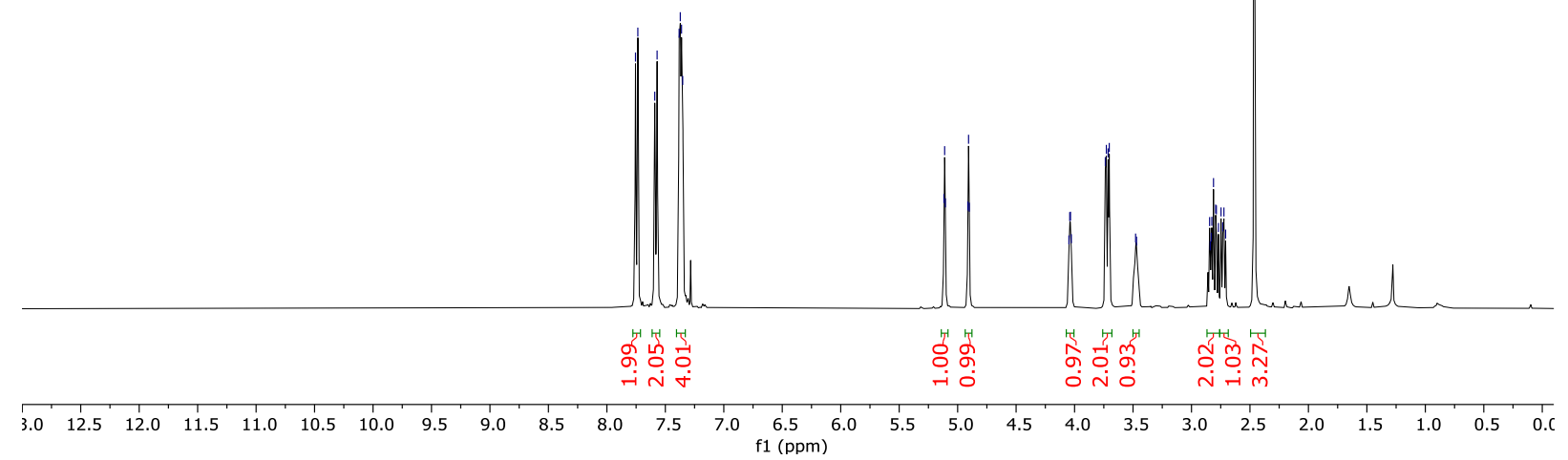

${ }^{13} \mathrm{C}$ NMR (101 MHz, $\mathrm{CDCl}_{3}$ )

AS180.4.1.1r

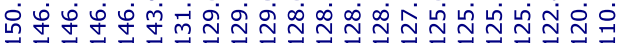

ำำกำㅇำ

กึ่

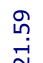

i

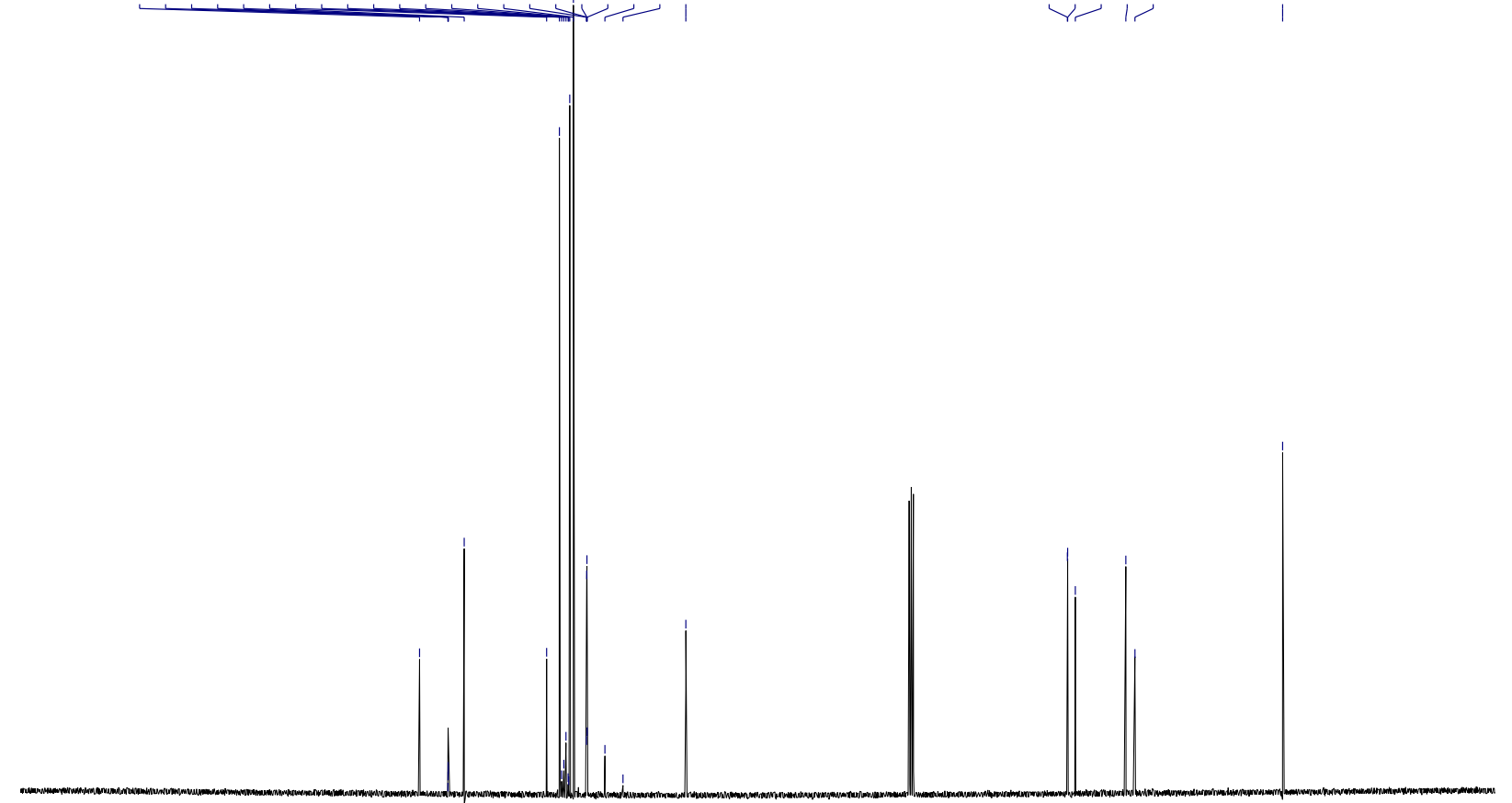

$\begin{array}{llllllllllllllllllllllllllll}10 & 200 & 190 & 180 & 170 & 160 & 150 & 140 & 130 & 120 & 110 & 100 & 90 & 80 & 70 & 60 & 50 & 40 & 30 & 20 & 10 & 0 & -:\end{array}$ 
${ }^{19} \mathrm{~F} \mathrm{NMR}\left(564 \mathrm{MHz}, \mathrm{CDCl}_{3}\right.$ )

AS180

single_pulse

$\stackrel{\infty}{\sim}$<smiles>C=C1[C@@H]2CN([As])C[C@@H]2[C@H]1c1ccc(C(F)(F)F)cc1</smiles>

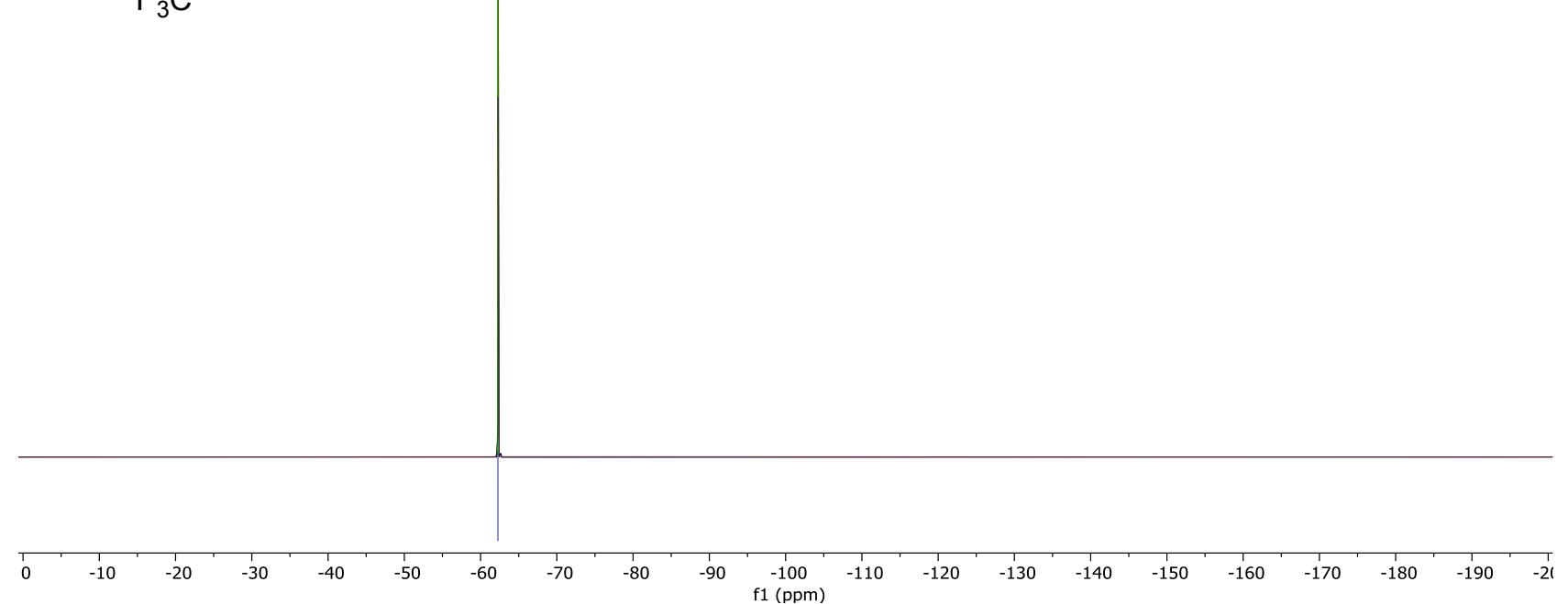


${ }^{1} \mathrm{H} \mathrm{NMR}\left(600 \mathrm{MHz}, \mathrm{CDCl}_{3}\right)$

AS1B 사수<smiles>[3H]N1C[C@@H]2C(=C)[C@H](c3cccc(F)c3)[C@@H]2C1</smiles>

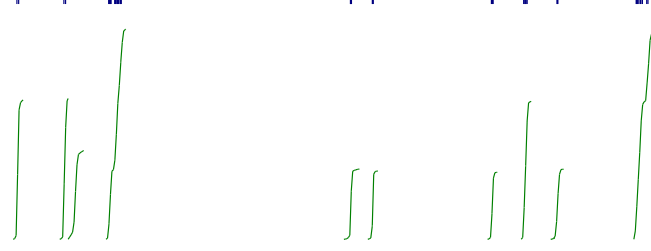

dd

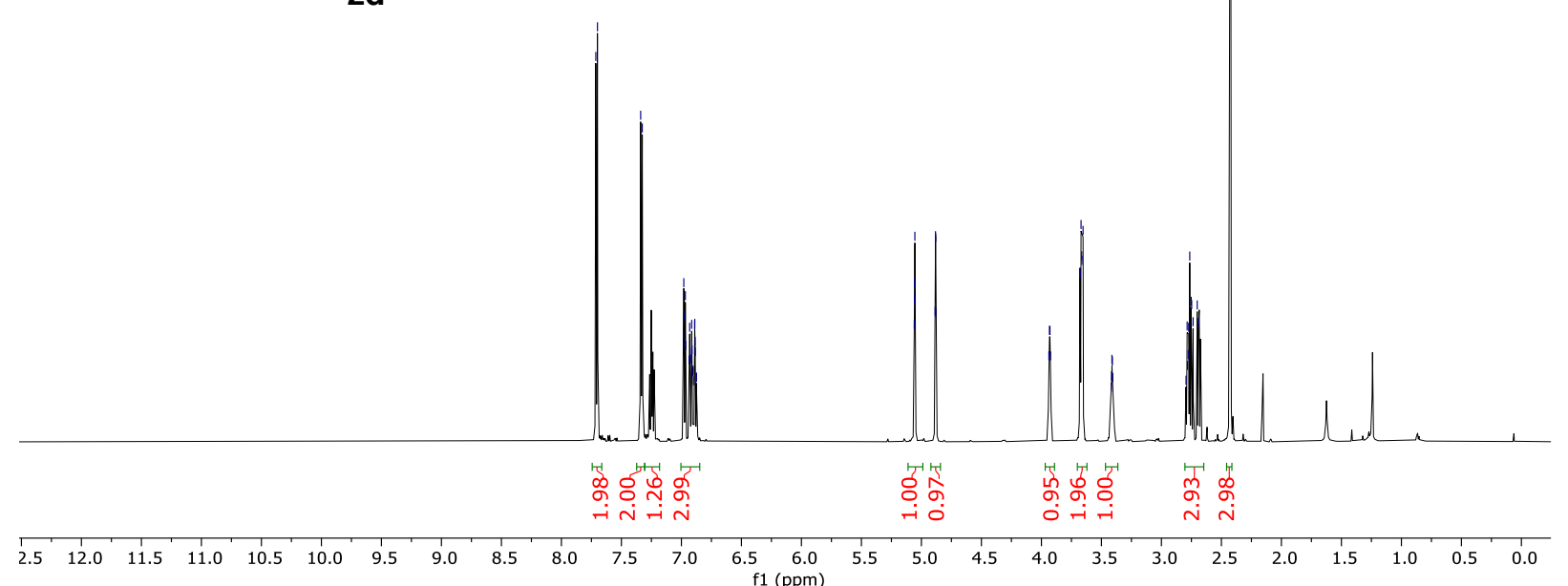

${ }^{13} \mathrm{C}$ NMR (151 MHz, $\left.\mathrm{CDCl}_{3}\right)$

AS182 药

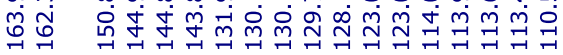

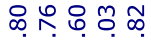

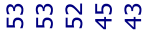

ָㅡㅁ

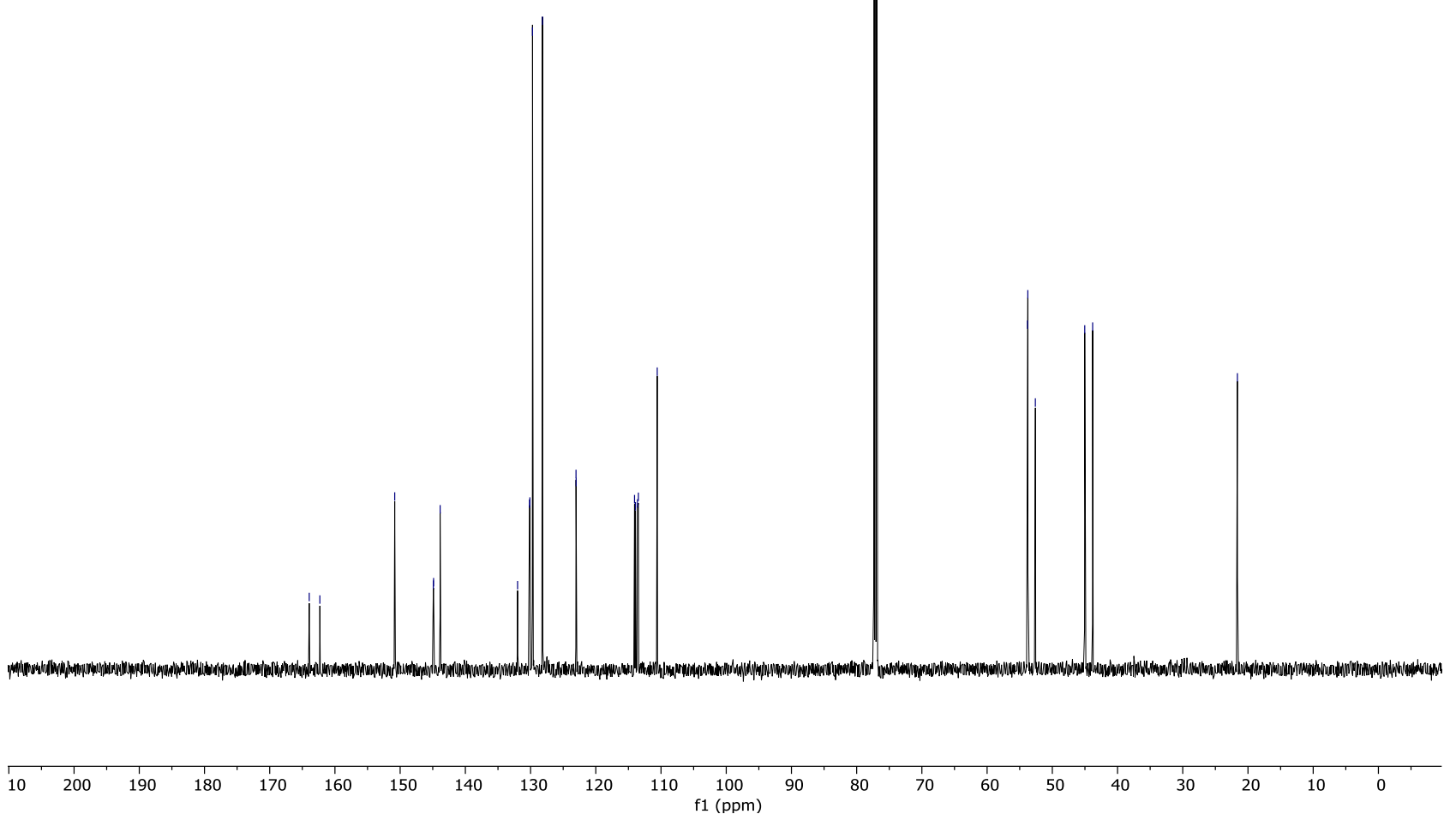


${ }^{19} \mathrm{~F} \mathrm{NMR} \mathrm{(564} \mathrm{MHz,} \mathrm{CDCl}_{3}$ )

AS182

ॠे

고 고 ㄱำ<smiles>[3H]N1C[C@H]2C(=C)[C@H](c3cccc(F)c3)[C@H]2C1</smiles>

2d

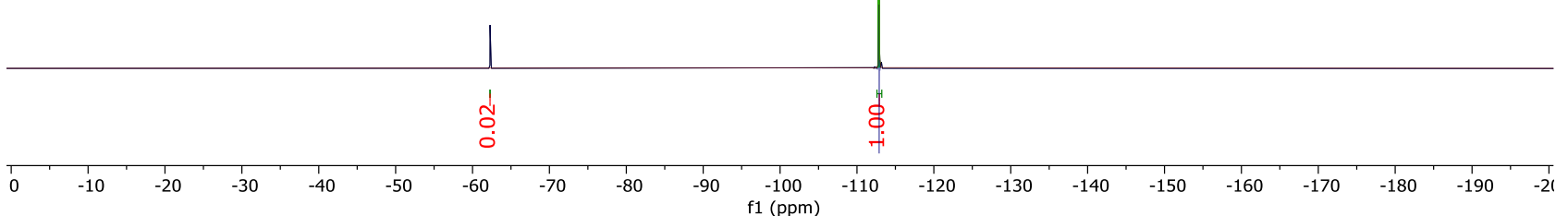


${ }^{1} \mathrm{H}$ NMR (400 MHz, $\mathrm{CDCl}_{3}$ )

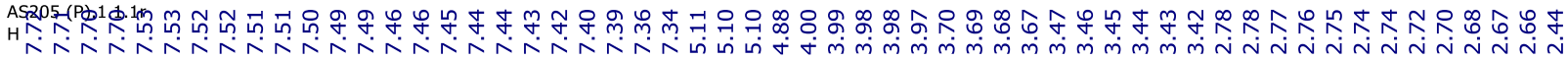<smiles>[3H]N1C[C@@H]2C(=C)[C@H](c3cccc(C#N)c3)[C@@H]2C1</smiles>
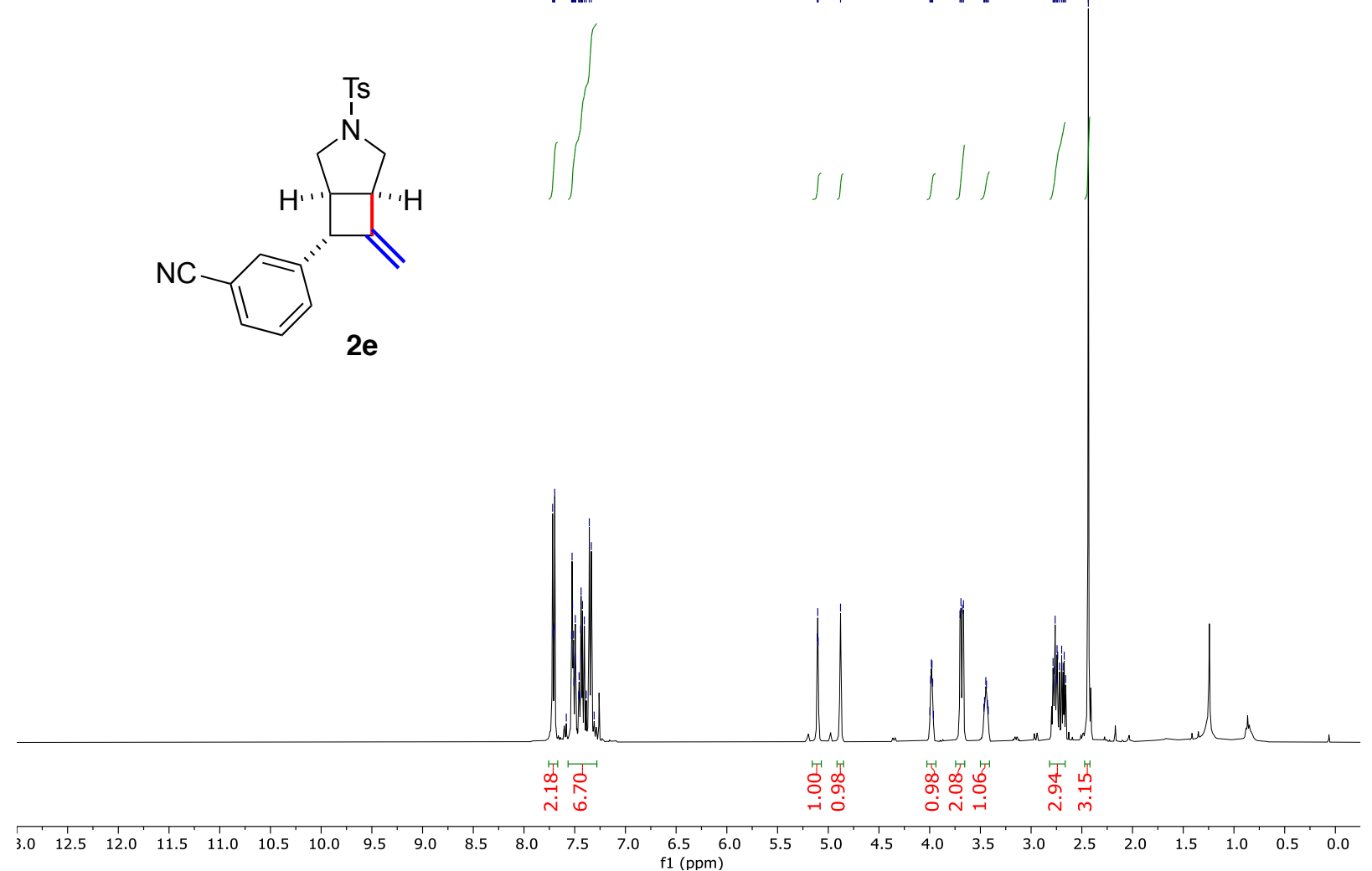

${ }^{13} \mathrm{C}$ NMR (101 MHz, $\mathrm{CDCl}_{3}$ )

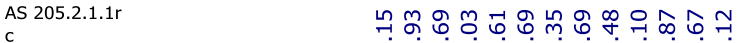

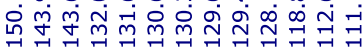

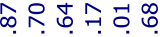

ดे ผึ่

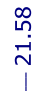

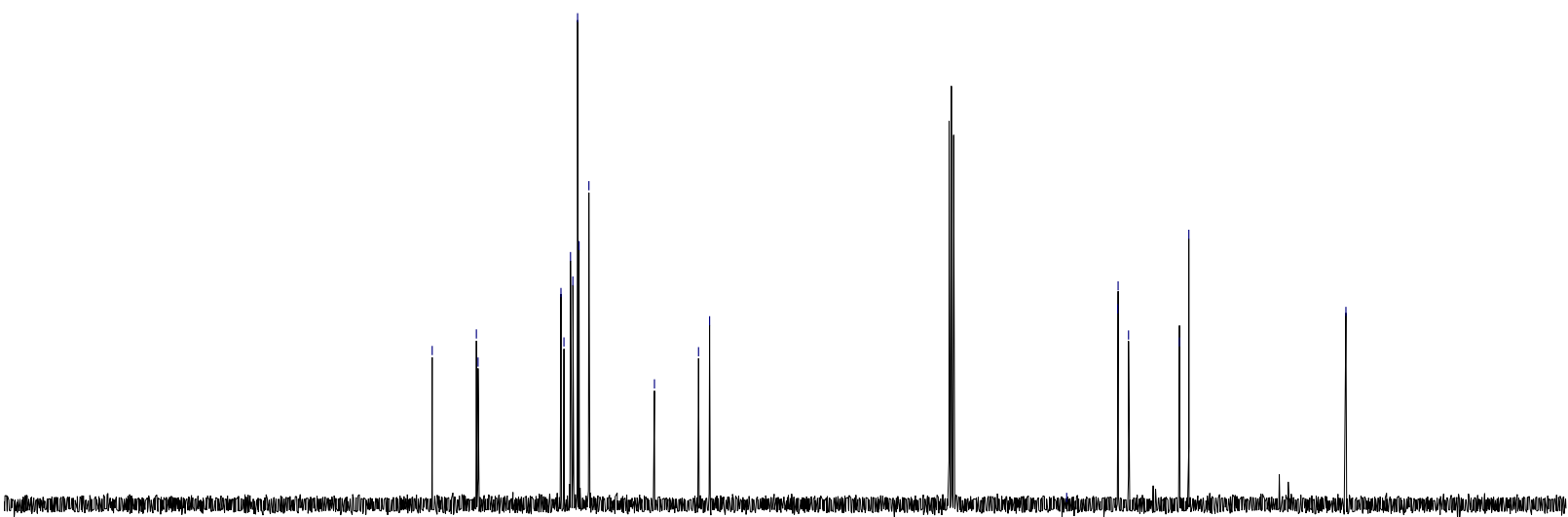

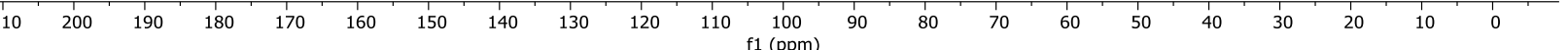


${ }^{1} \mathrm{H}$ NMR $\left(400 \mathrm{MHz}, \mathrm{CDCl}_{3}\right)$

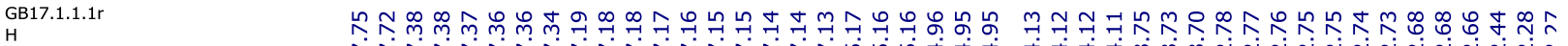

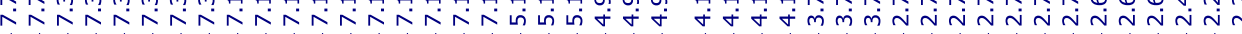<smiles>C=C1[C@H]2CN([AsH3])C[C@@H]2[C@H]1c1ccccc1C</smiles>

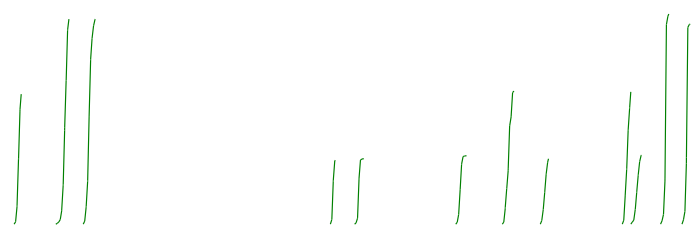

$2 f$

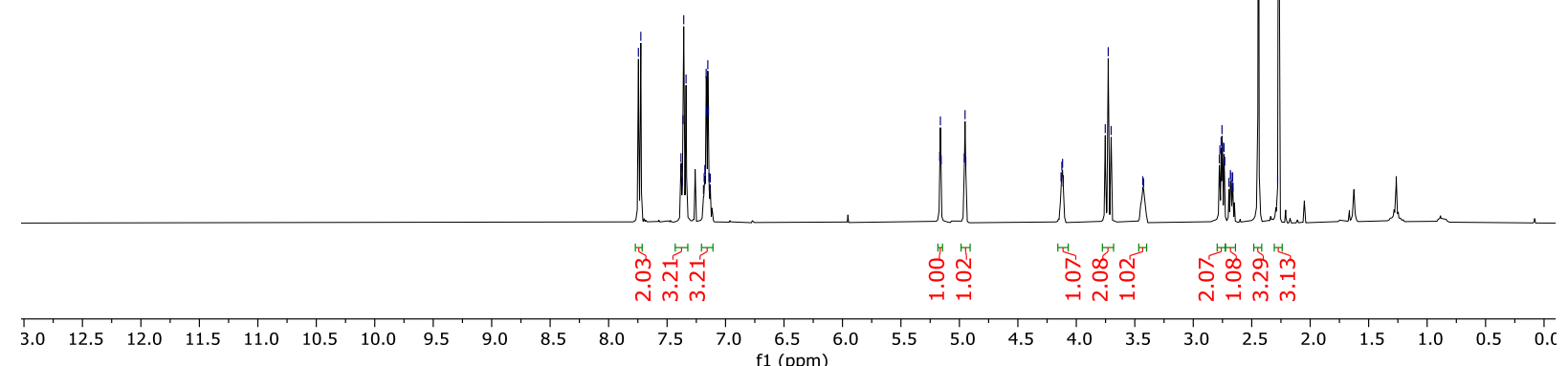

\section{${ }^{13} \mathrm{C}$ NMR (101 MHz, $\left.\mathrm{CDCl}_{3}\right)$}

GB17.2.1.1r

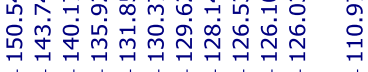

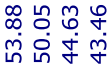

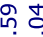

4.

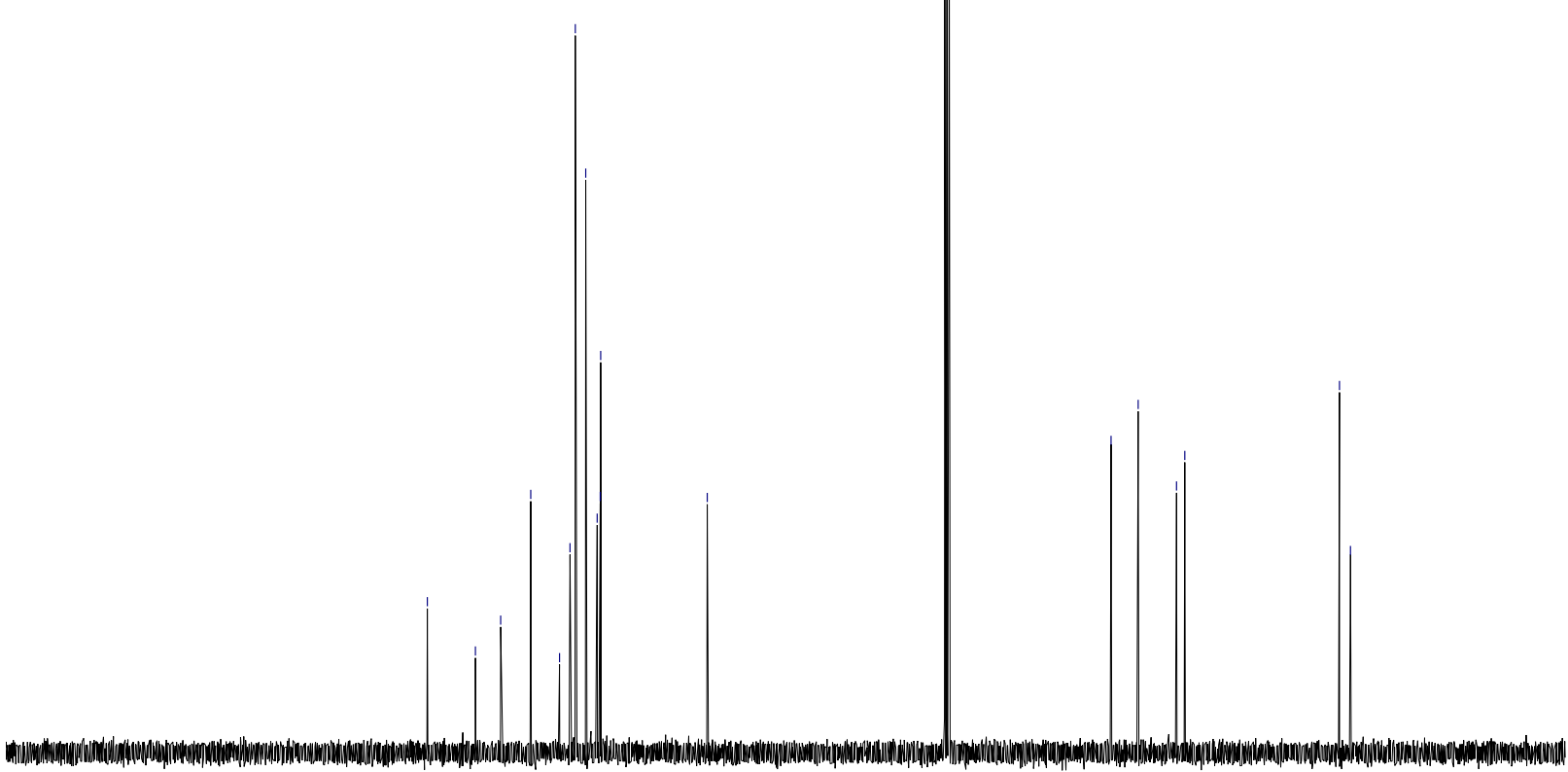

$\begin{array}{lllllllllllllllllllllllll}10 & 200 & 190 & 180 & 170 & 160 & 150 & 140 & 130 & 120 & 110 & \begin{array}{c}100 \\ \mathrm{f} 1(\mathrm{ppm})\end{array} & 90 & 80 & 70 & 60 & 50 & 40 & 30 & 20 & 10 & 0 & -1\end{array}$ 
${ }^{1} \mathrm{H}$ NMR (400 MHz, $\left.\mathrm{CDCl}_{3}\right)$

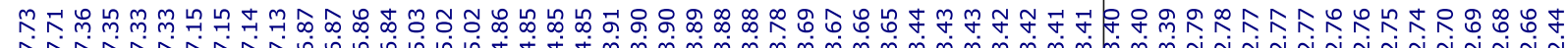

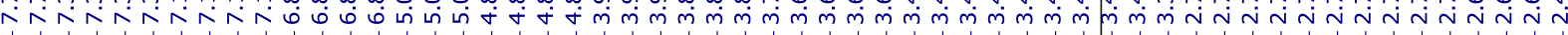<smiles>[3H]N1CC2C(=C)[C@H](c3ccc(OC)cc3)[C@@H]2C1</smiles>

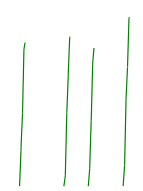

2g

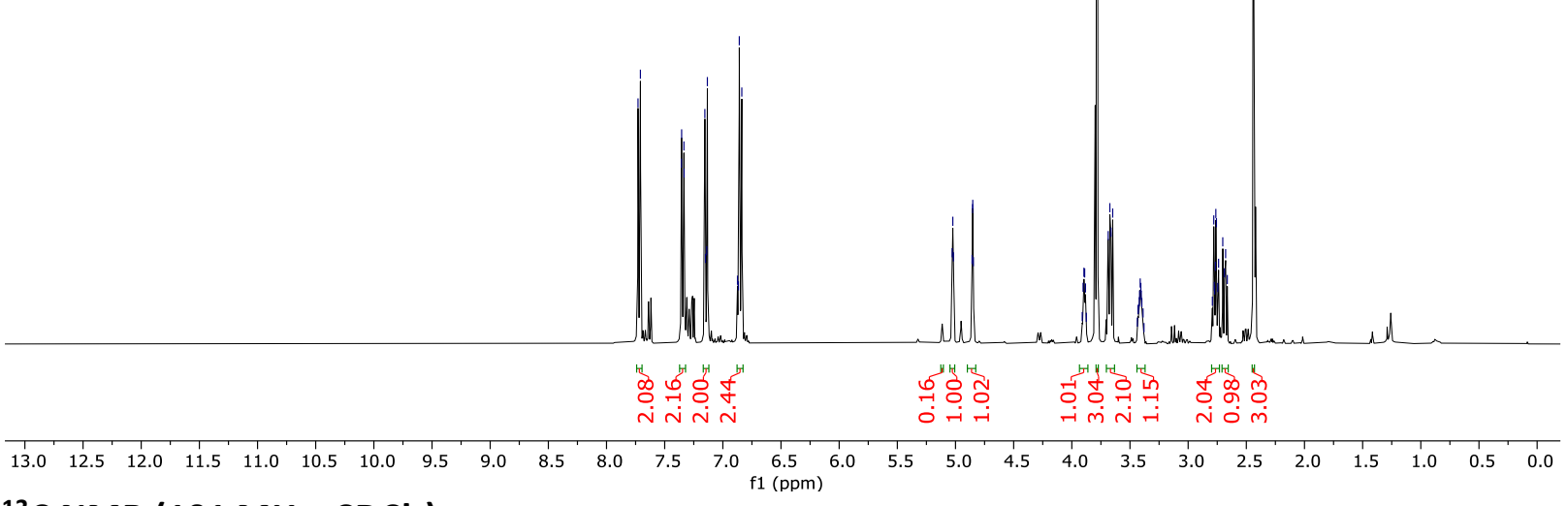

${ }^{13} \mathrm{C}$ NMR (101 MHz, $\left.\mathrm{CDCl}_{3}\right)$

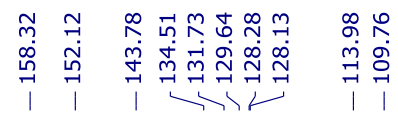

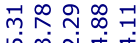

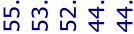

$\stackrel{\vec{n}}{+}$

1) i

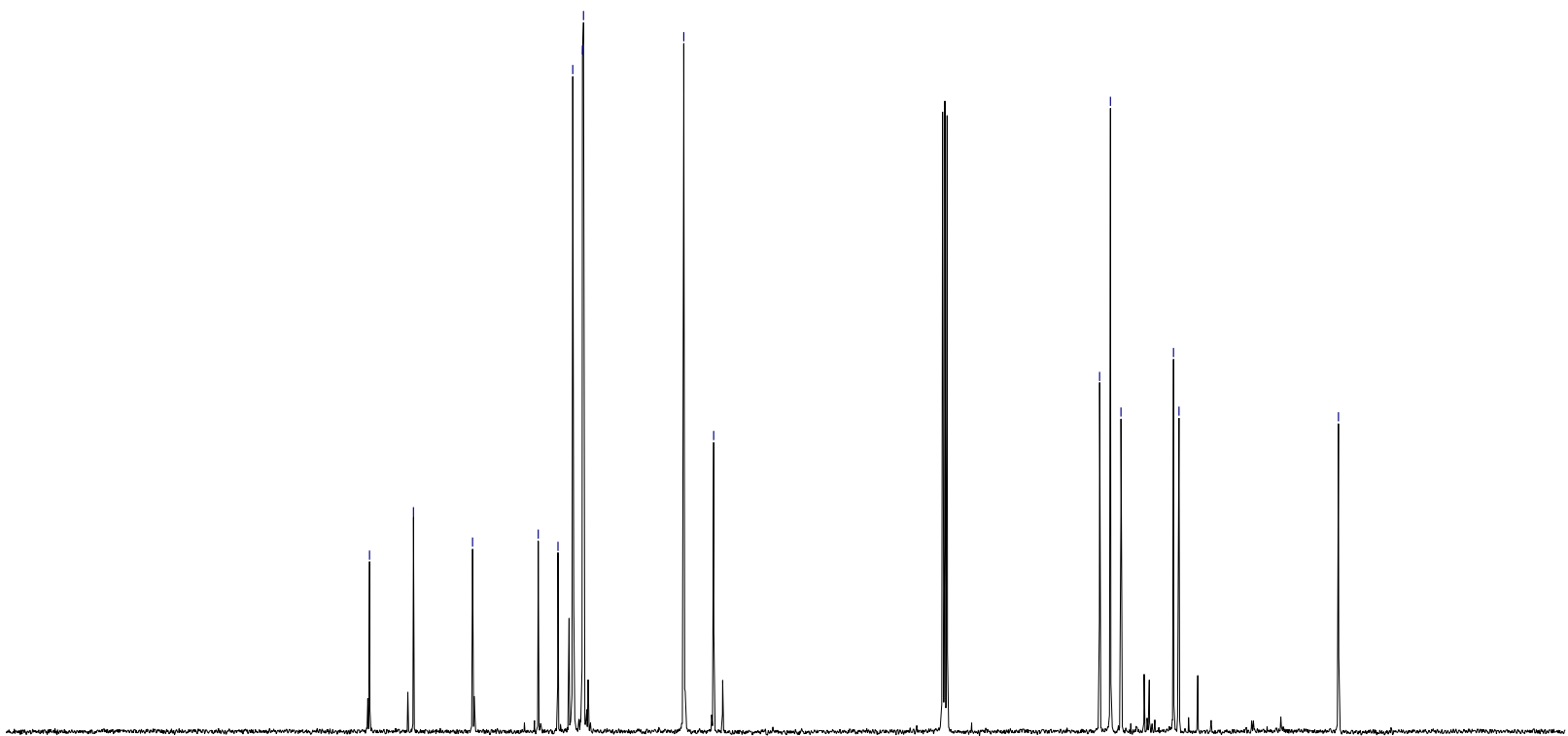

$\begin{array}{lllllllllllllllllllllll}200 & 190 & 180 & 170 & 160 & 150 & 140 & 130 & 120 & 110 & 100 & 90 & 80 & 70 & 60 & 50 & 40 & 30 & 20 & 10 & 0 & -1\end{array}$ 
${ }^{1} \mathrm{H}$ NMR (400 MHz, $\left.\mathrm{CDCl}_{3}\right)$

AS244 pulito.1.fid

오ํ mั

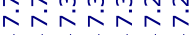

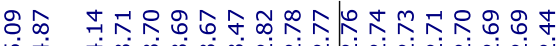

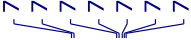

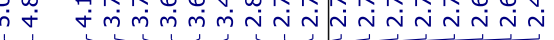<smiles>C=C1[C@H]2CN([AsH3])C[C@H]2[C@H]1c1cccnc1</smiles>

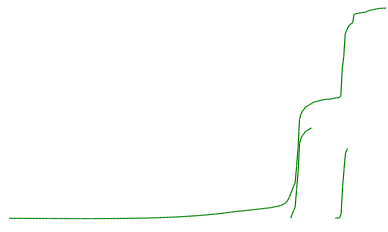

$2 \mathrm{~h}$

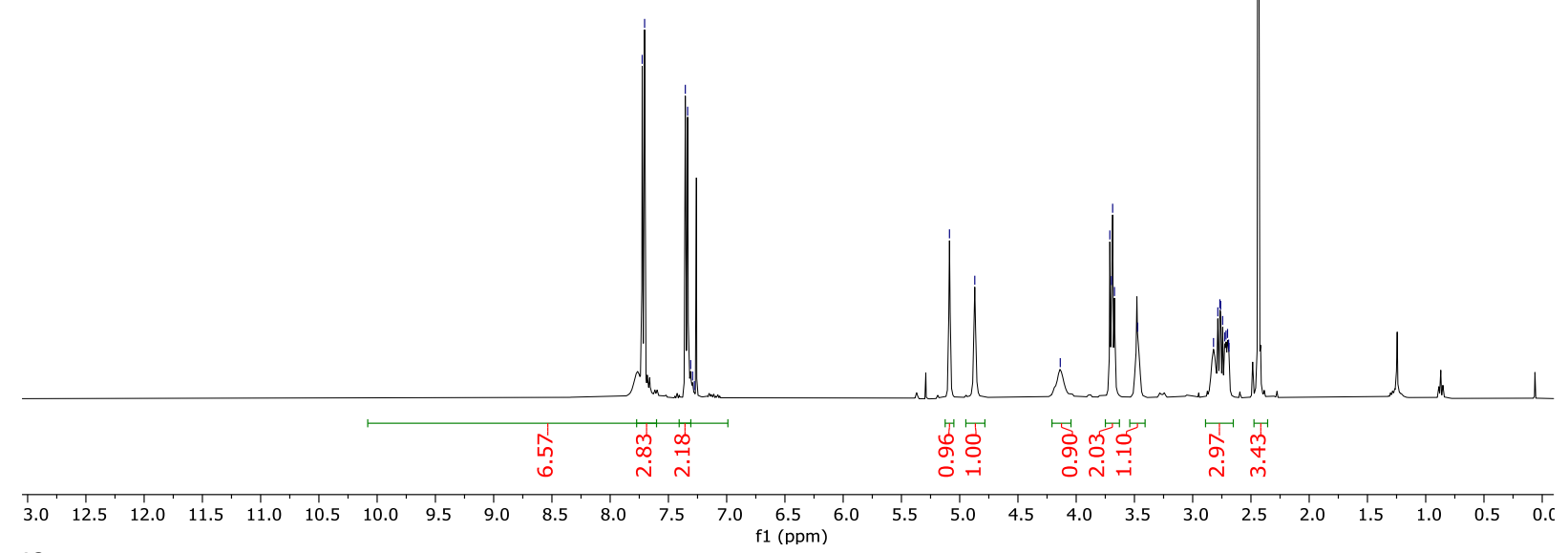

${ }^{13} \mathrm{C}$ NMR (101 MHz, $\mathrm{CDCl}_{3}$ )

AS244 pulito.2.fid

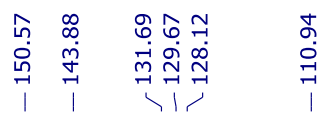

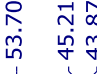

$\stackrel{8}{\mathrm{i}}$

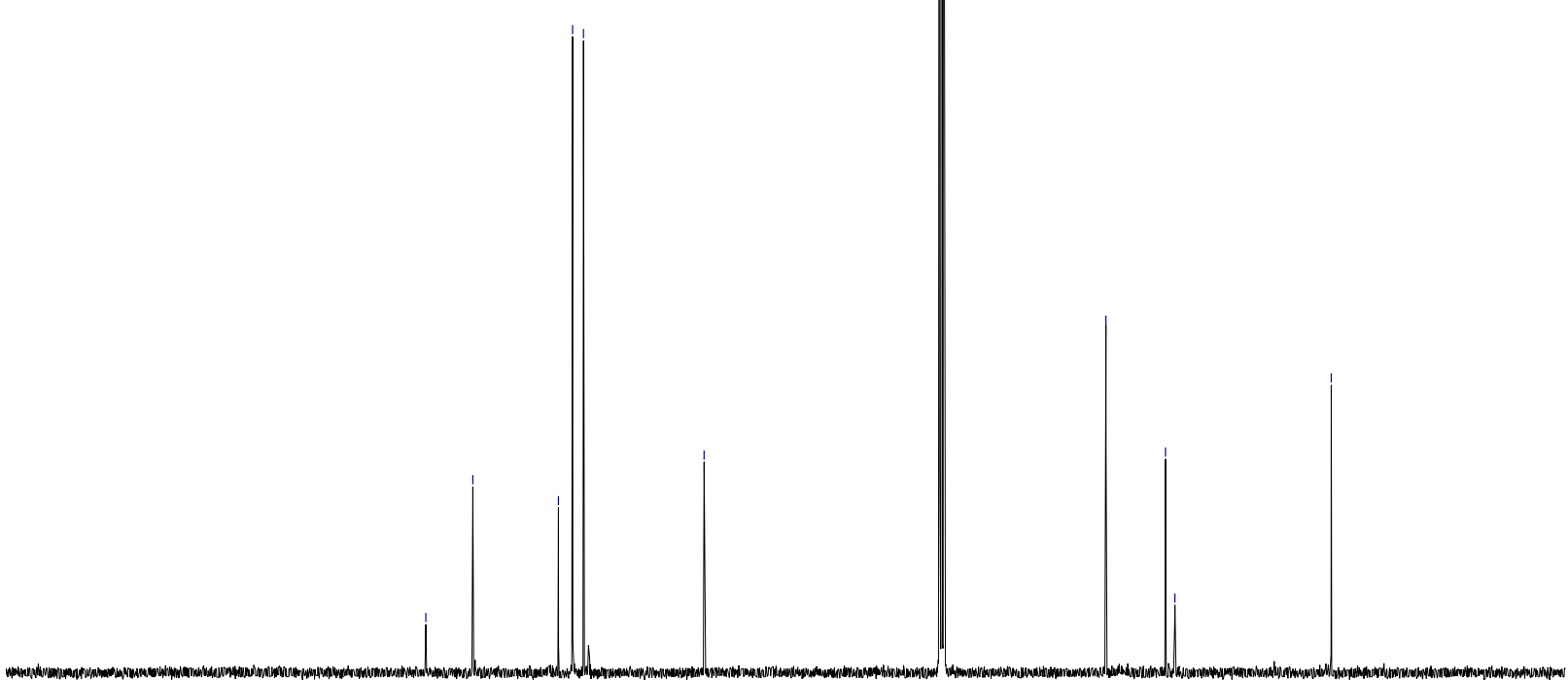

$\begin{array}{llllllllllllllllllllllll}10 & 200 & 190 & 180 & 170 & 160 & 150 & 140 & 130 & 120 & 110 & 100 & 90 & 80 & 70 & 60 & 50 & 40 & 30 & 20 & 10 & 0 & -1\end{array}$ 
${ }^{1} \mathrm{H}$ NMR $\left(400 \mathrm{MHz}, \mathrm{CDCl}_{3}\right)$

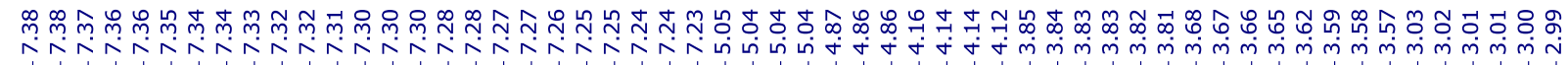

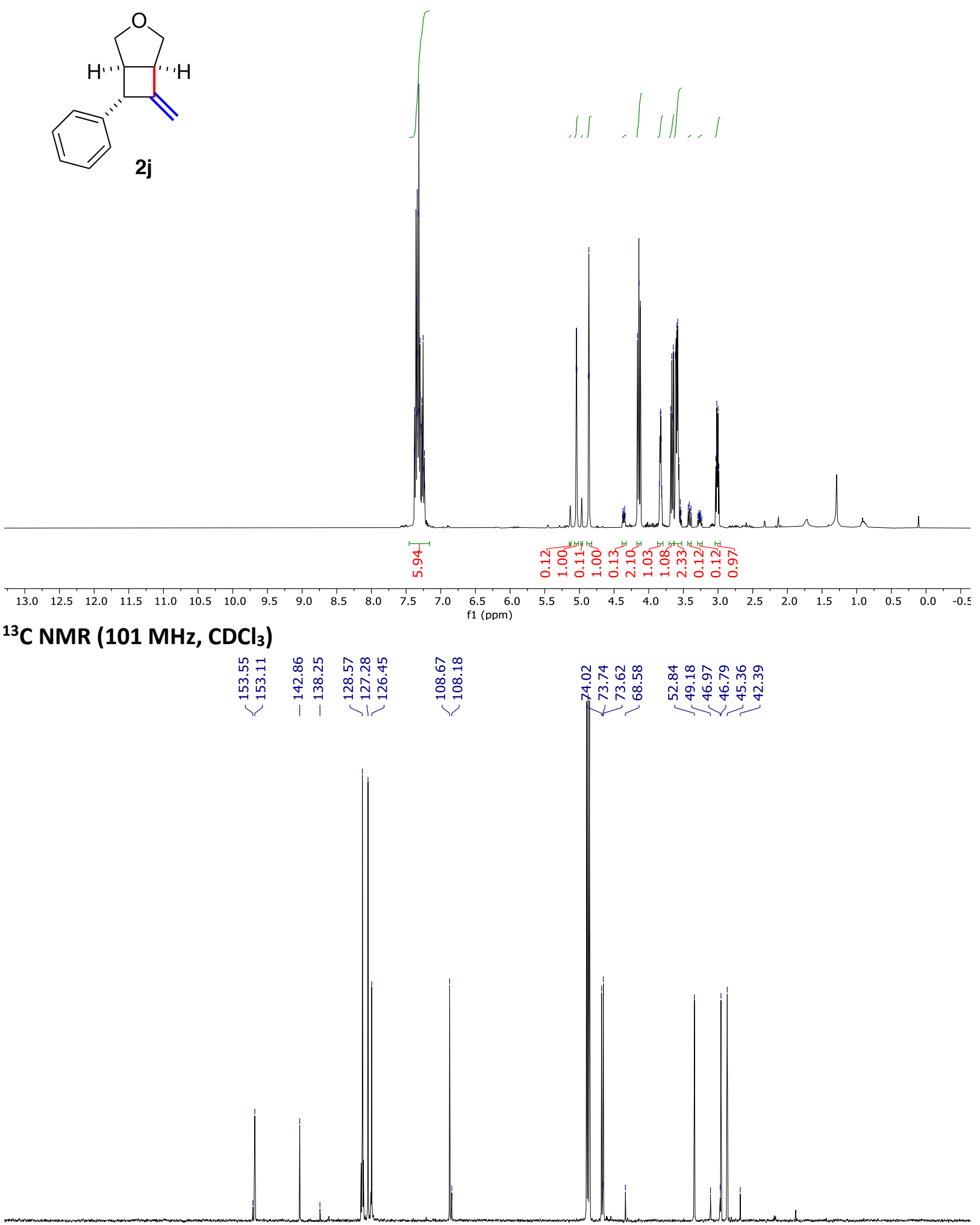

$\begin{array}{llllllllllllllllllllllllllllll}10 & 200 & 190 & 180 & 170 & 160 & 150 & 140 & 130 & 120 & 110 & 100 & 90 & 80 & 70 & 60 & 50 & 40 & 30 & 20 & 10 & 0 & -1\end{array}$ 
${ }^{1} \mathrm{H}$ NMR (400 MHz, $\left.\mathrm{CDCl}_{3}\right)$

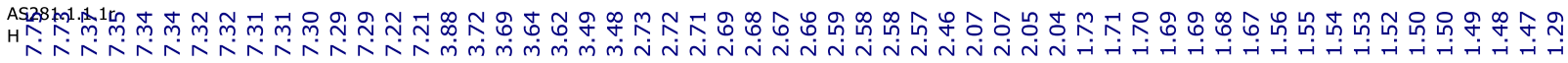<smiles>[NH-]C1C[C@@H]2[C@@H](c3ccccc3)C(=C3CCCCC3)[C@@H]2C1</smiles>

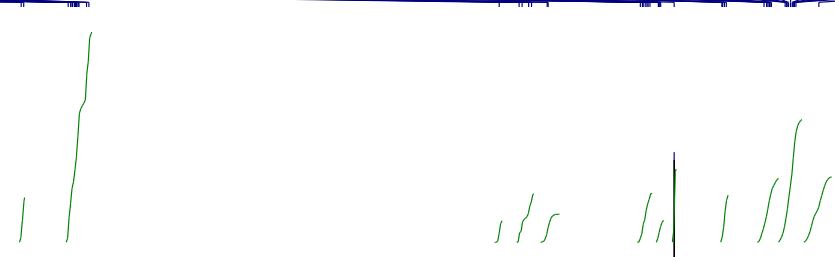

2k

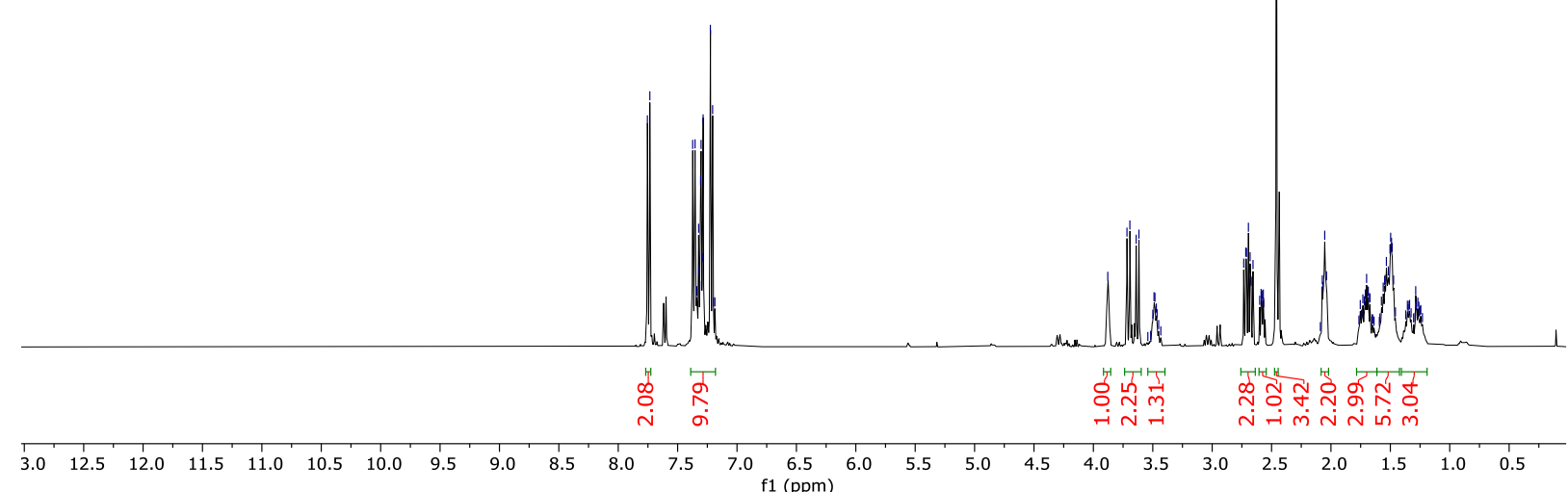

${ }^{13} \mathrm{C}$ NMR (101 MHz, CDCl 3 )

AS281.2.1.1r
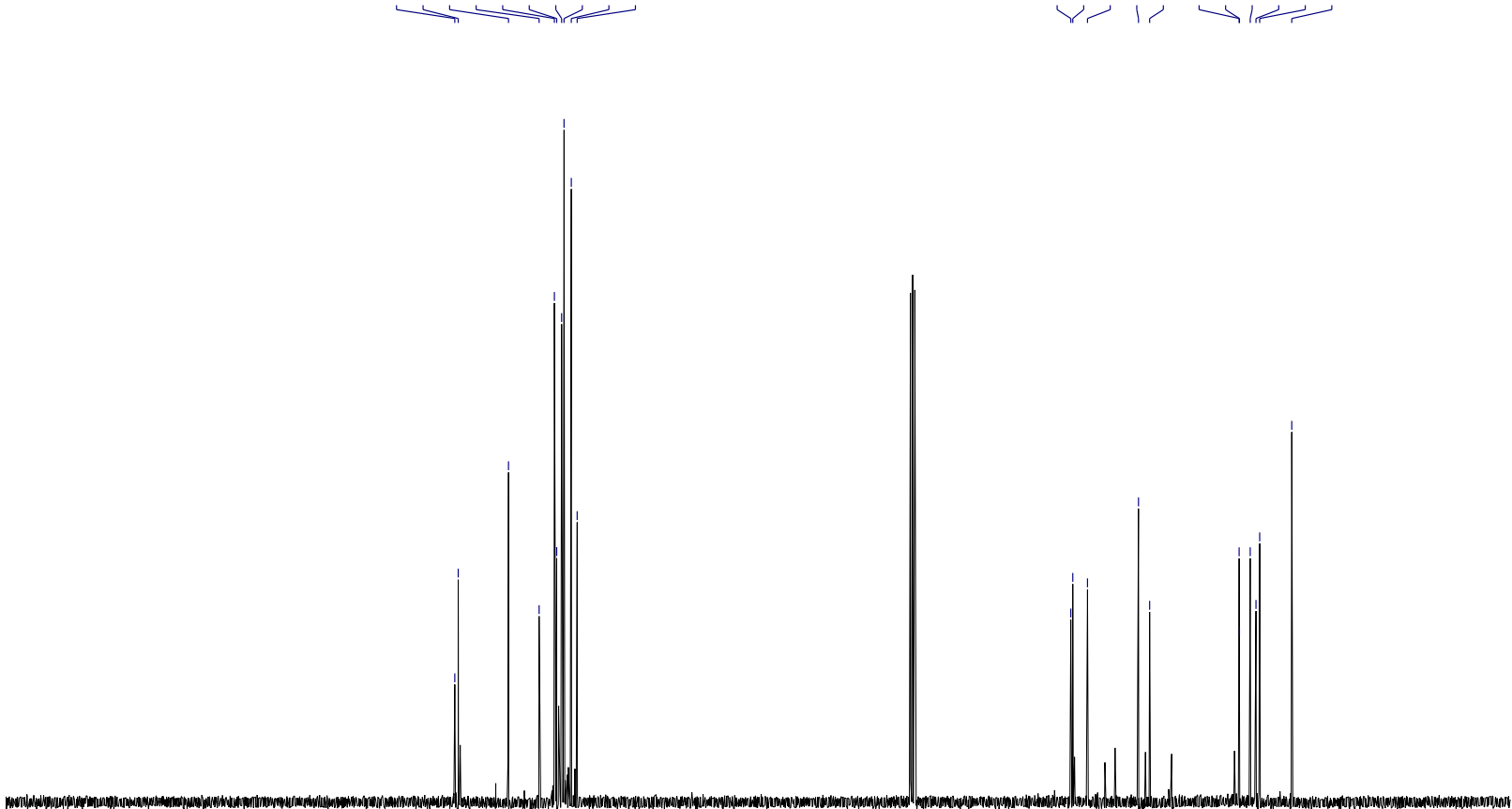

$\begin{array}{llllllllllllllllllllllll}200 & 190 & 180 & 170 & 160 & 150 & 140 & 130 & 120 & 110 & 100 & 90 & 80 & 70 & 60 & 50 & 40 & 30 & 20 & 10 & 0 & -1\end{array}$ 
${ }^{1} \mathrm{H}$ NMR (400 MHz, acetone)

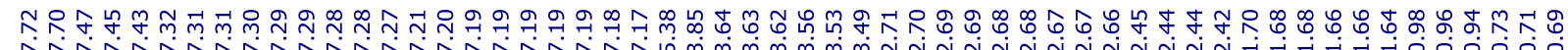

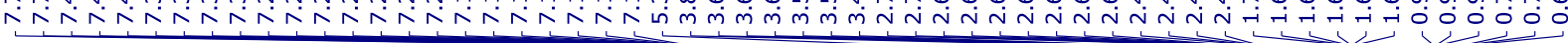<smiles>CCC=C1[C@H]2CN([AsH3])C[C@H]2[C@H]1c1ccccc1</smiles>
L

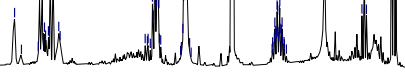

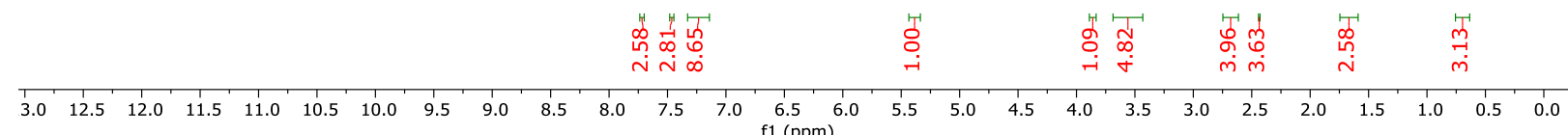

${ }^{13} \mathrm{C}$ NMR (101 MHz, acetone)

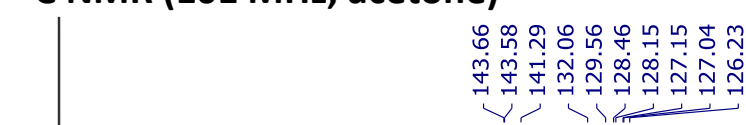

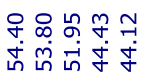

รุำ ภำ

กิ่

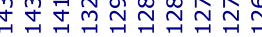

140 $10 \begin{gathered}100 \\ \text { f1 }(\mathrm{ppm})\end{gathered}$ 
${ }^{1} \mathrm{H}$ NMR (400 MHz, $\left.\mathrm{CDCl}_{3}\right)$

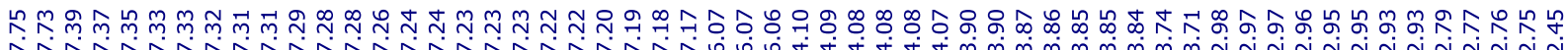

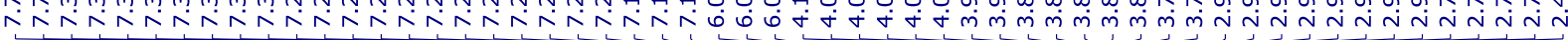

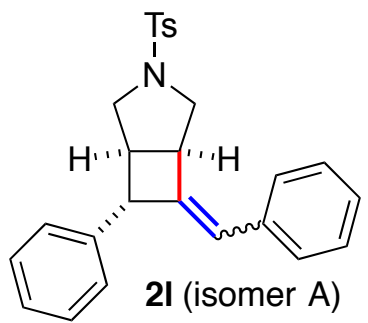
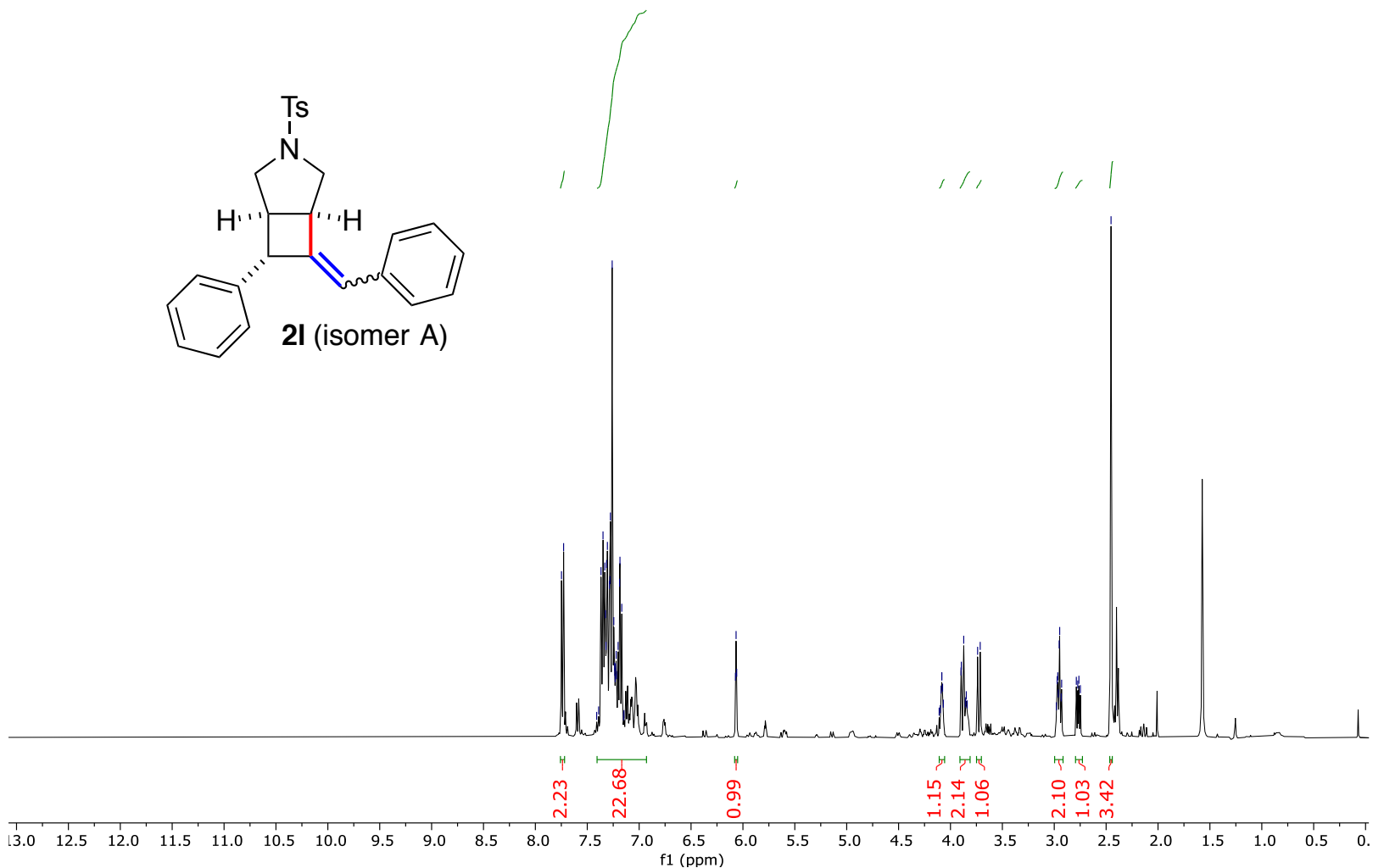

${ }^{13} \mathrm{C}$ NMR (101 MHz, $\left.\mathrm{CDCl}_{3}\right)$

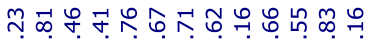

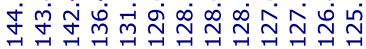

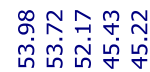

$\widehat{\sigma}$

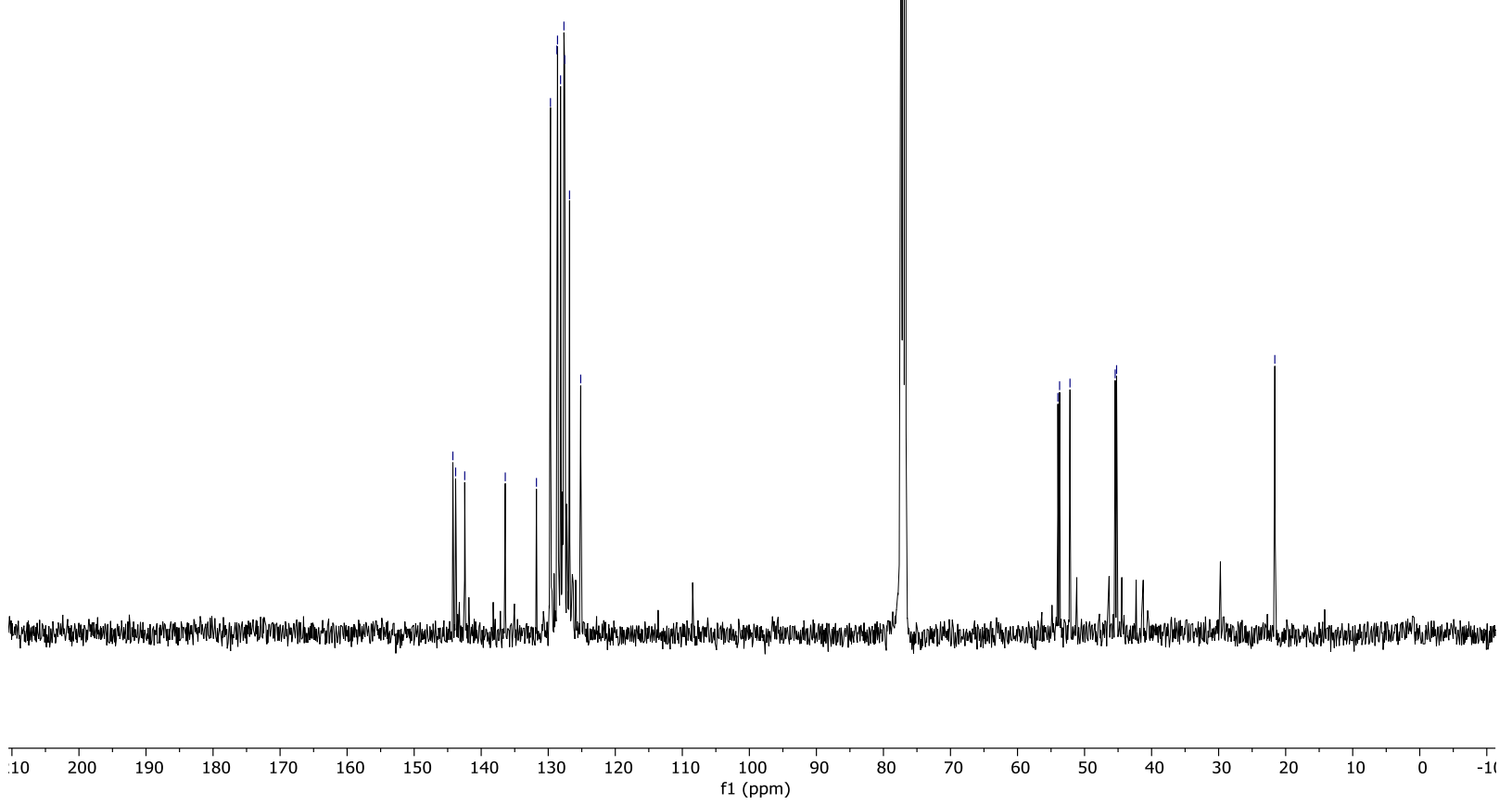


${ }^{1} \mathrm{H}$ NMR (400 MHz, $\left.\mathrm{CDCl}_{3}\right)$

As212Hapdod

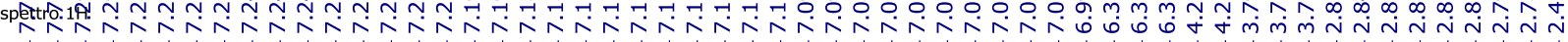

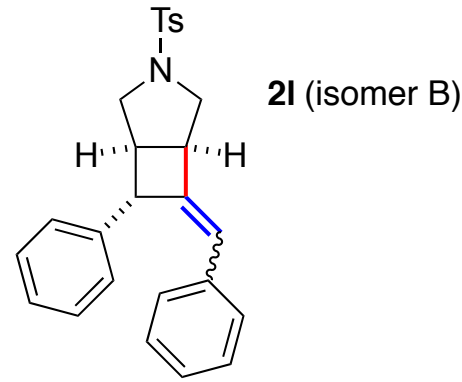<smiles>CC1CCCCC1</smiles>

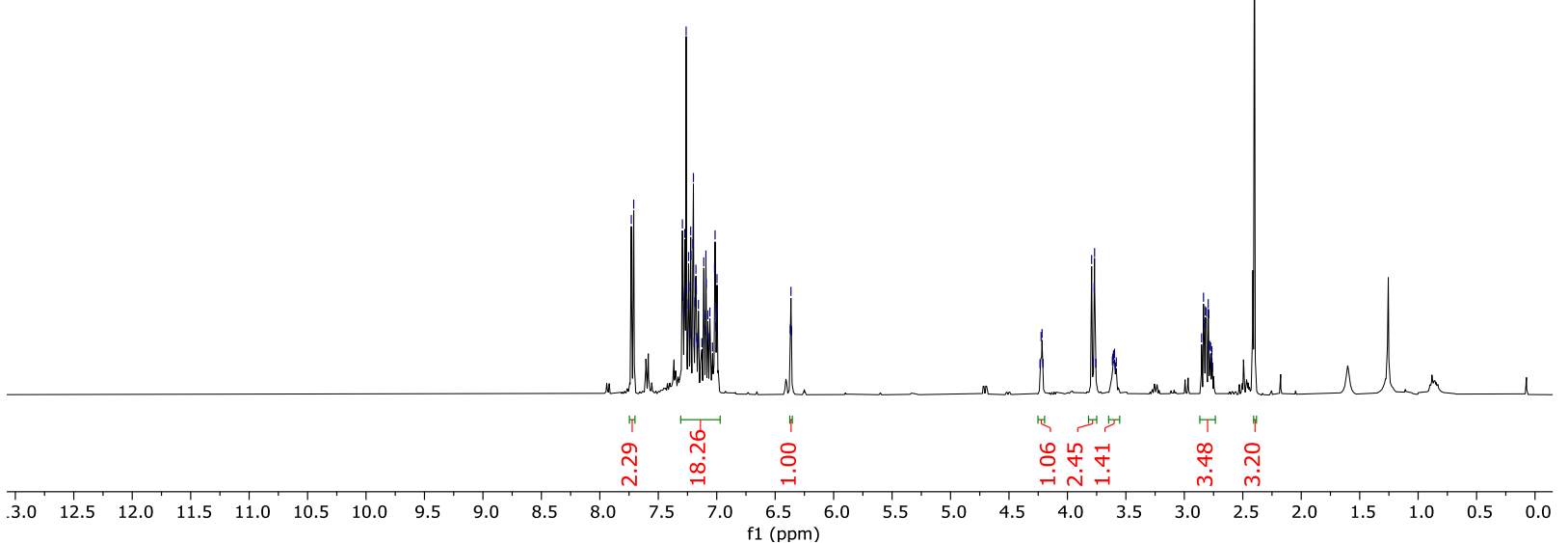

\section{${ }^{13} \mathrm{C}$ NMR (101 MHz, $\left.\mathrm{CDCl}_{3}\right)$}

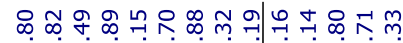

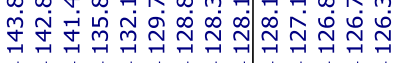

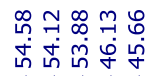

$\stackrel{\infty}{\stackrel{\infty}{+}}$

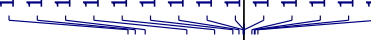

ro

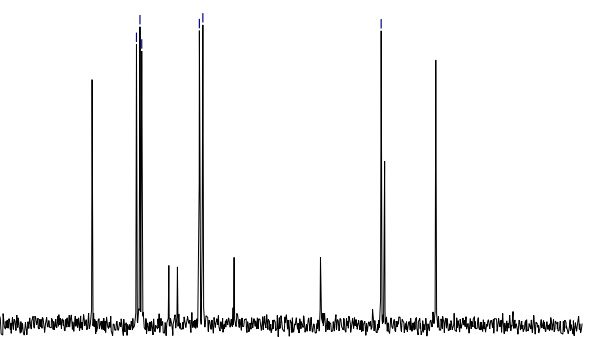

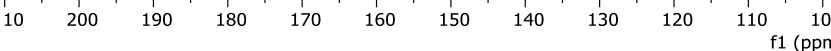


${ }^{1} \mathrm{H}$ NMR (600 MHz, $\mathrm{CDCl}_{3}$ )

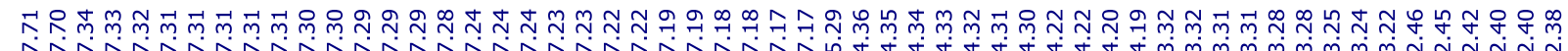

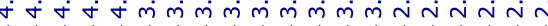
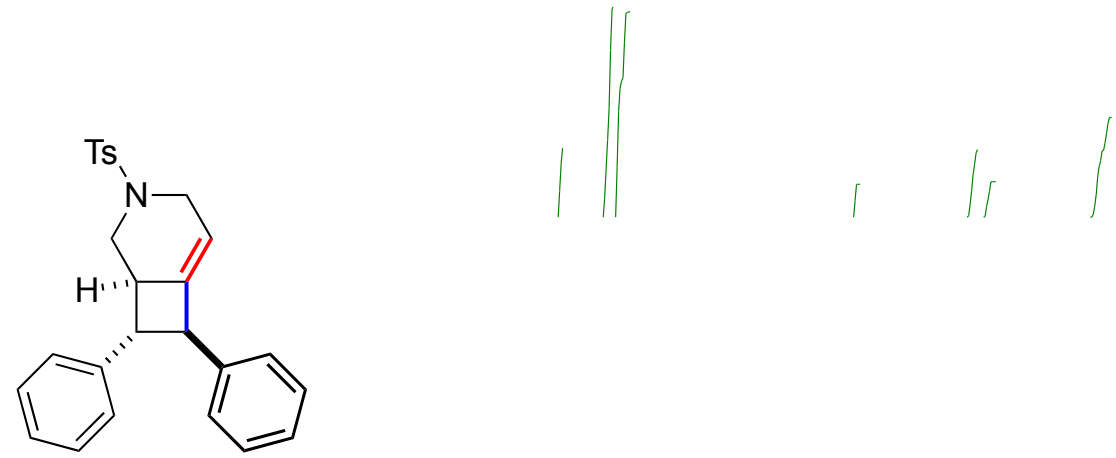

2'-I

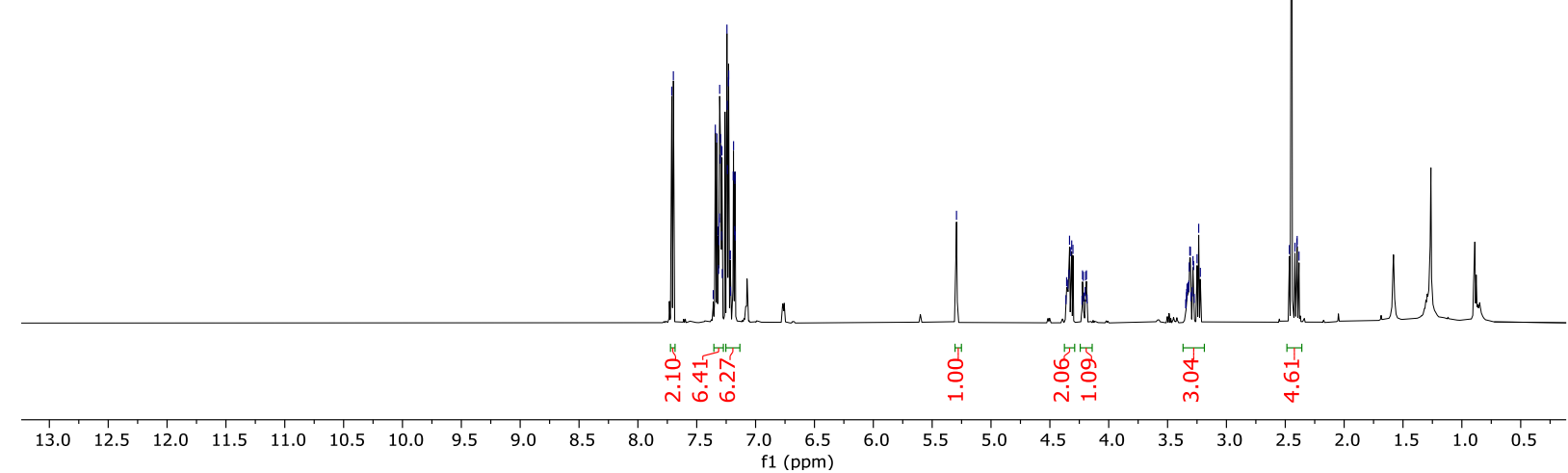

\section{${ }^{13} \mathrm{C}$ NMR (151 MHz, $\left.\mathrm{CDCl}_{3}\right)$}

ஸ்

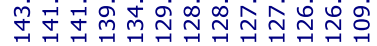

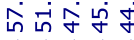

$\stackrel{\circ}{i}$

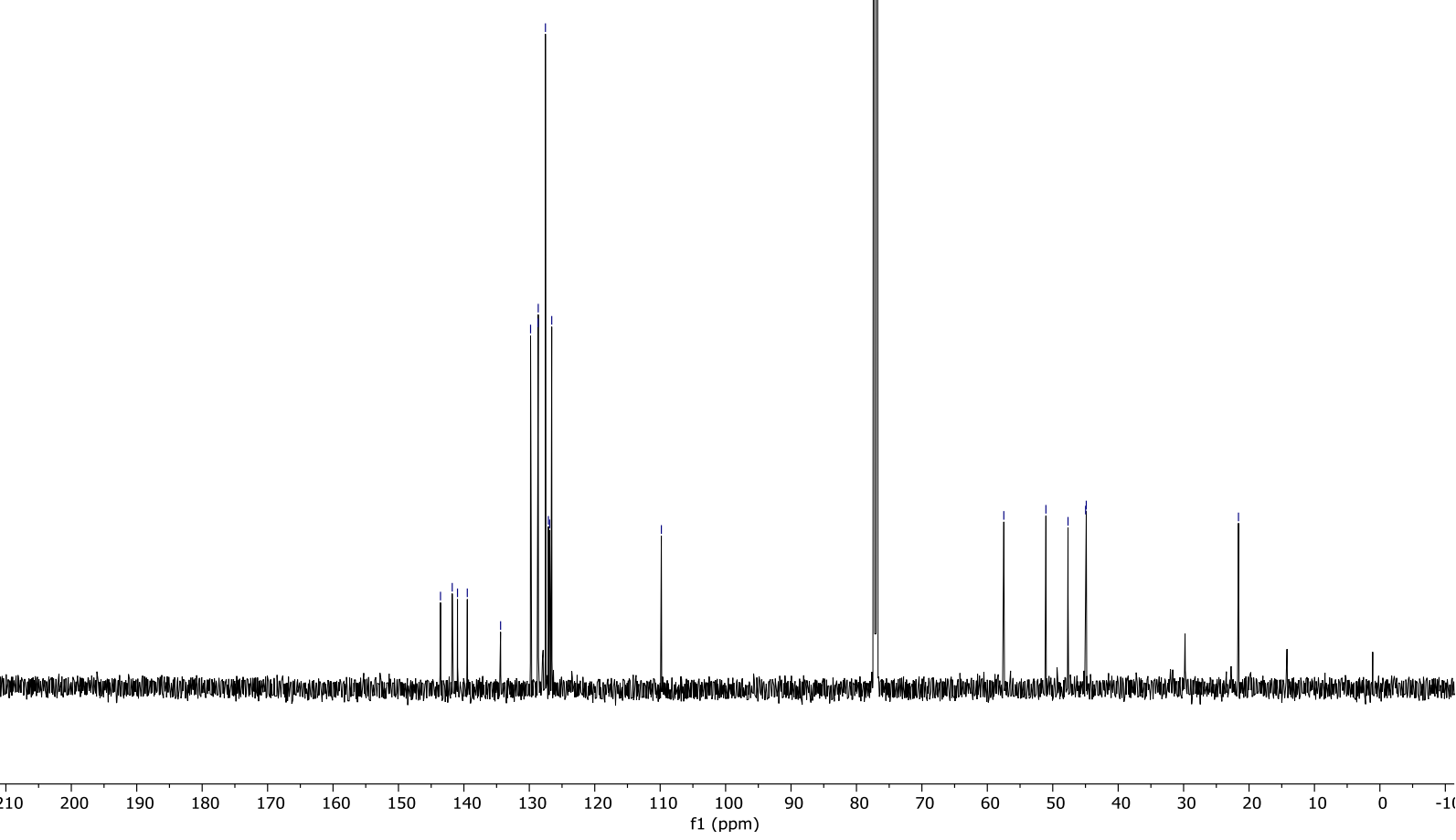


${ }^{1} \mathrm{H}$ NMR $\left(400 \mathrm{MHz}, \mathrm{CDCl}_{3}\right)$

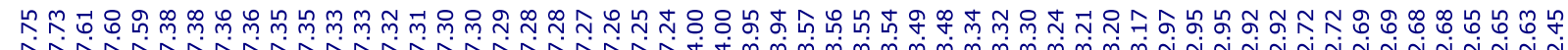

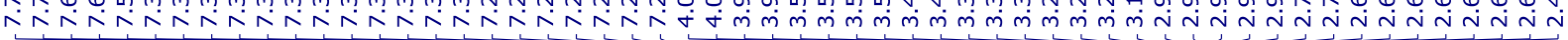<smiles>Cc1ccc([S@@]2(=O)C[C@H](c3ccccc3)[C@H]3CN=C[C@H]32)cc1</smiles><smiles>C=CCCC</smiles>

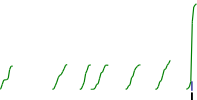

$4 a$

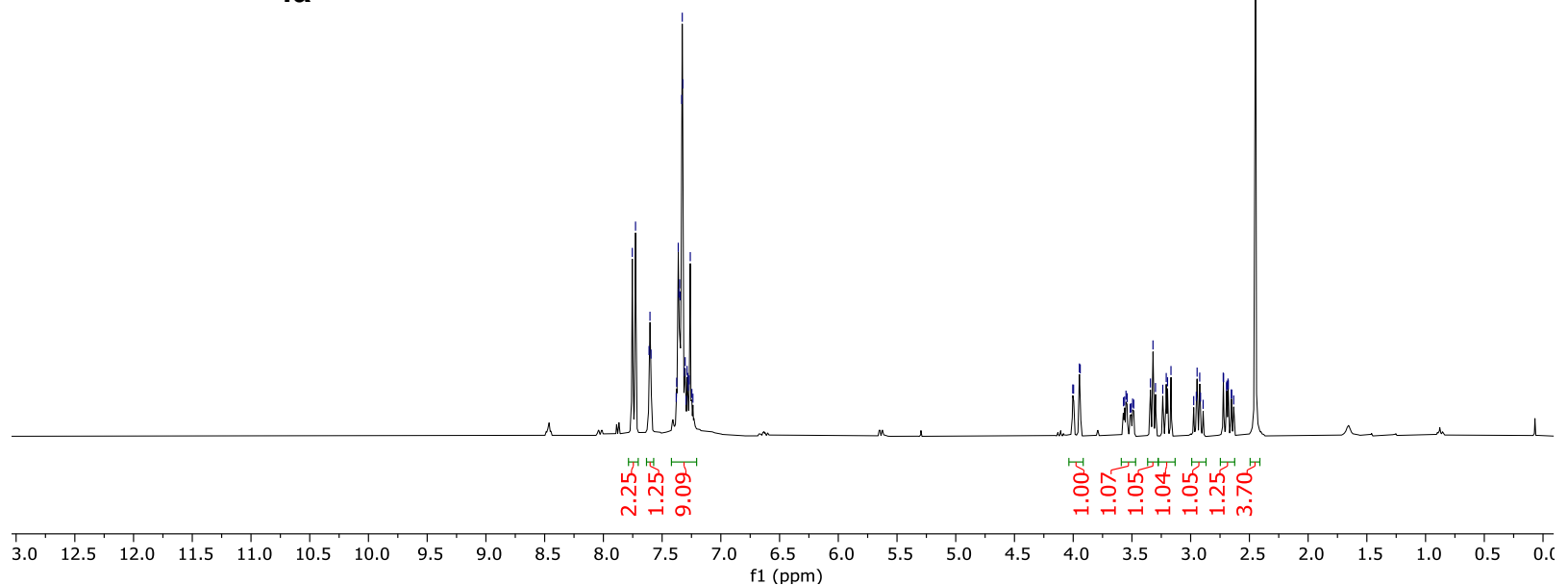

${ }^{13} \mathrm{C}$ NMR (101 MHz, $\mathrm{CDCl}_{3}$ )

AS99 12-17.2.fid

J $\quad$ 운

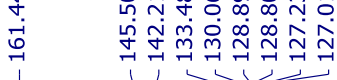

$\stackrel{\infty}{\circ}$

굴유
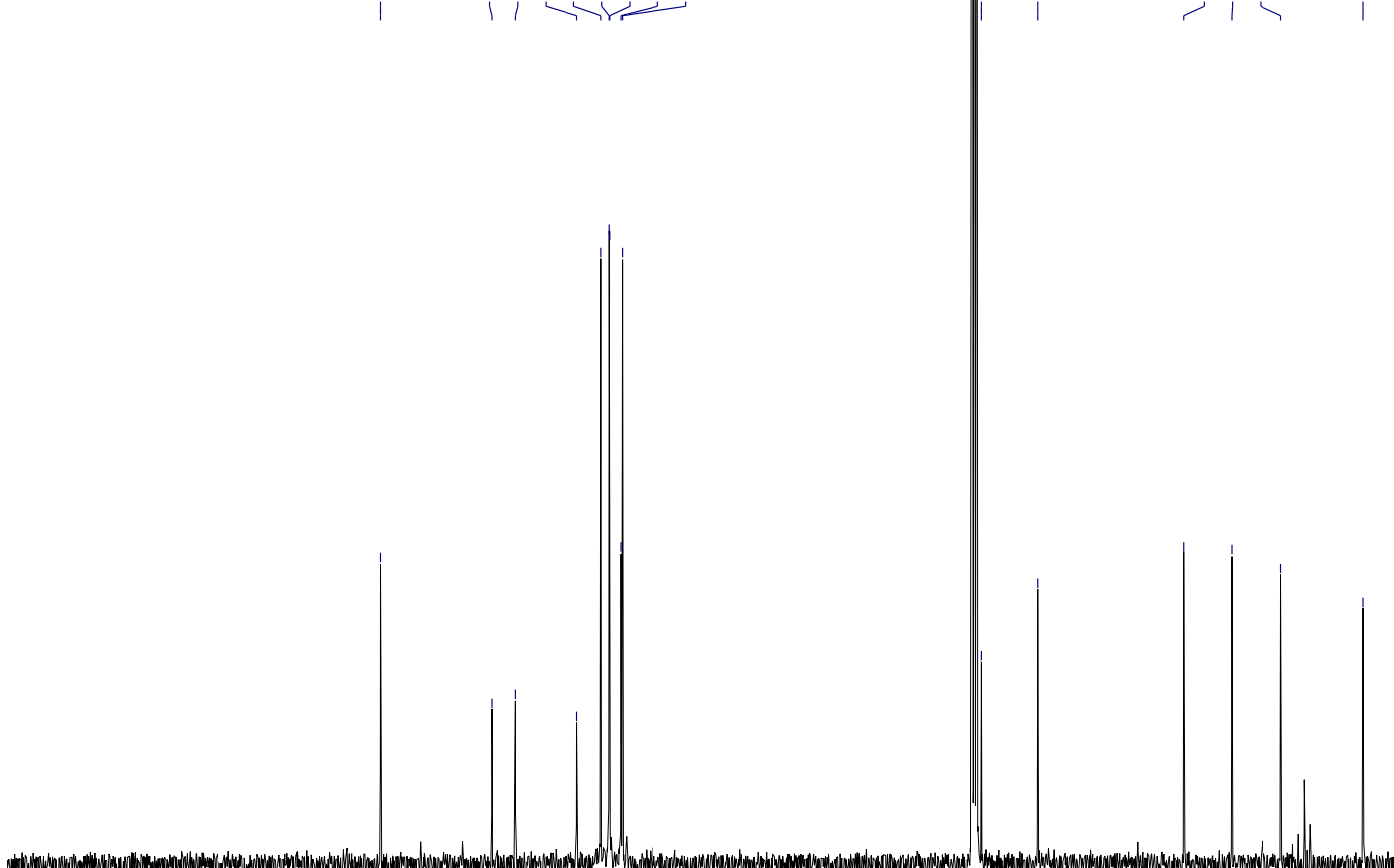

$210 \quad 200 \quad 190 \quad 180 \quad 170 \quad 160$ 100
f1 $(\mathrm{ppm})$ 守年 
${ }^{1} \mathrm{H}$ NMR (400 MHz, $\mathrm{CDCl}_{3}$ )

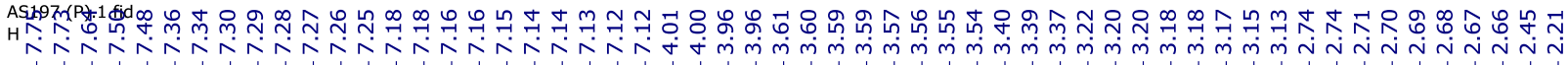<smiles>Cc1ccc([C@]2(O)C[C@@H]3[C@H]2C=NC[C@H]3c2ccccc2C)cc1</smiles>

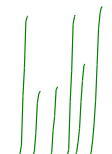

$4 b$

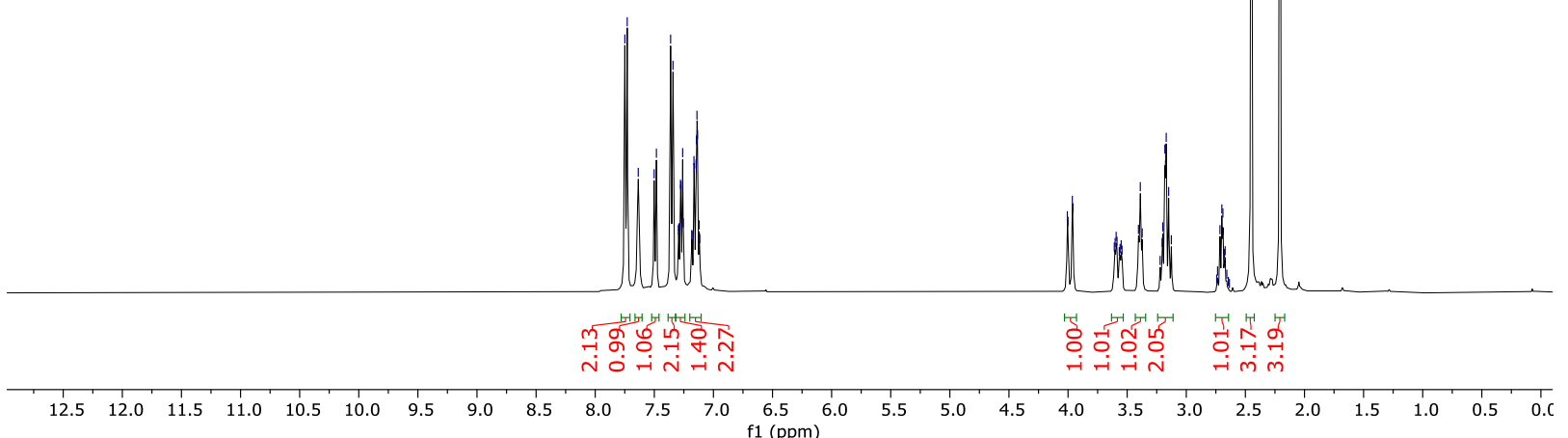

${ }^{13} \mathrm{C}$ NMR (101 MHz, $\mathrm{CDCl}_{3}$ )

AS197 (P).2.1.1r

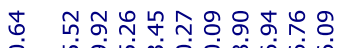

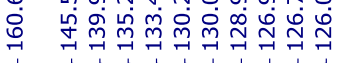

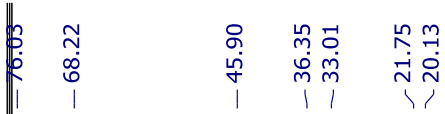

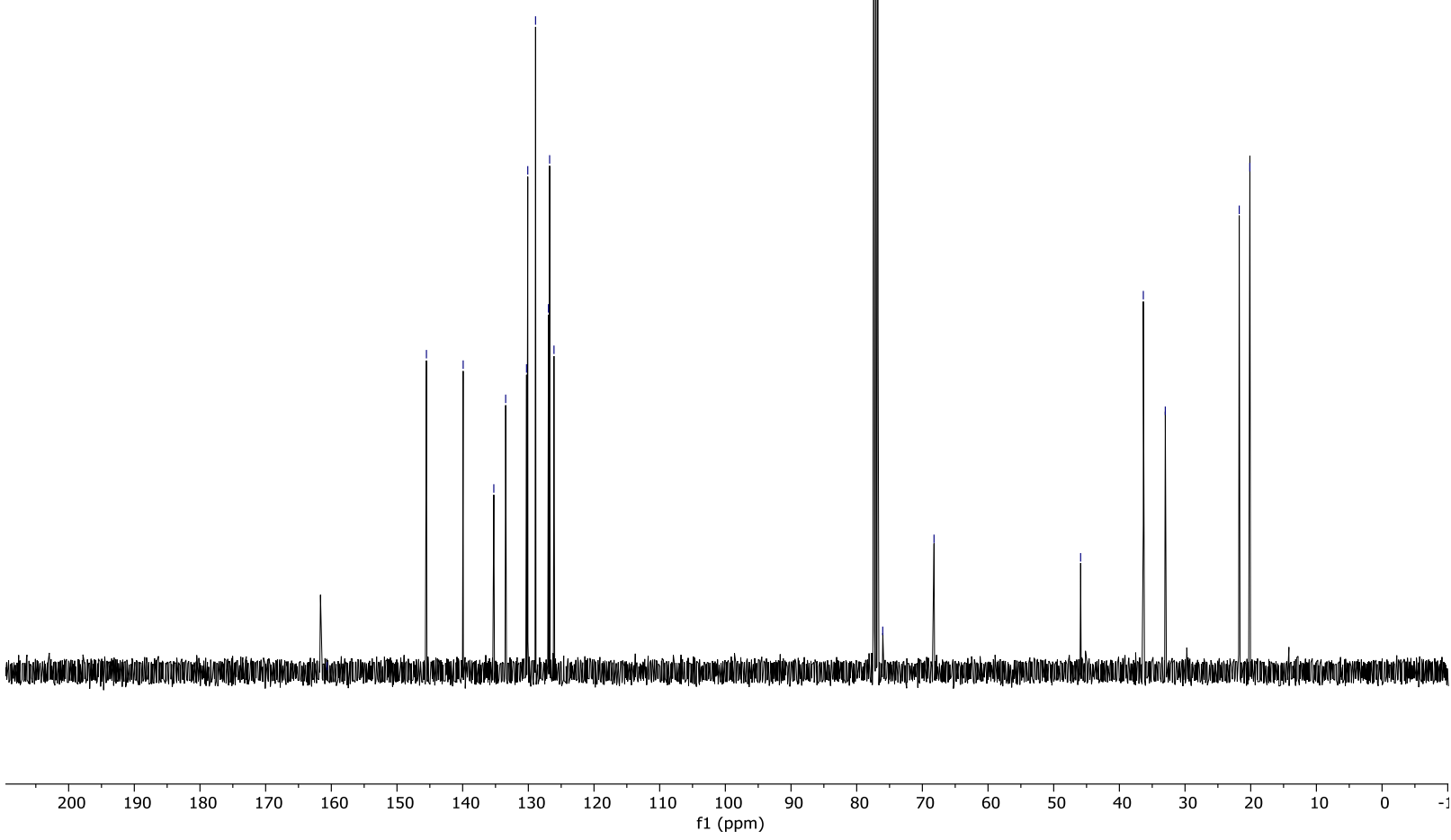


${ }^{1} \mathrm{H}$ NMR (300 MHz, $\left.\mathrm{CDCl}_{3}\right)$ AS149 H e C.1.fid
H<smiles>COc1ccc([C@@H]2C[C@]3(S(=O)(=O)c4ccc(C)cc4)C=NC[C@H]23)cc1</smiles>
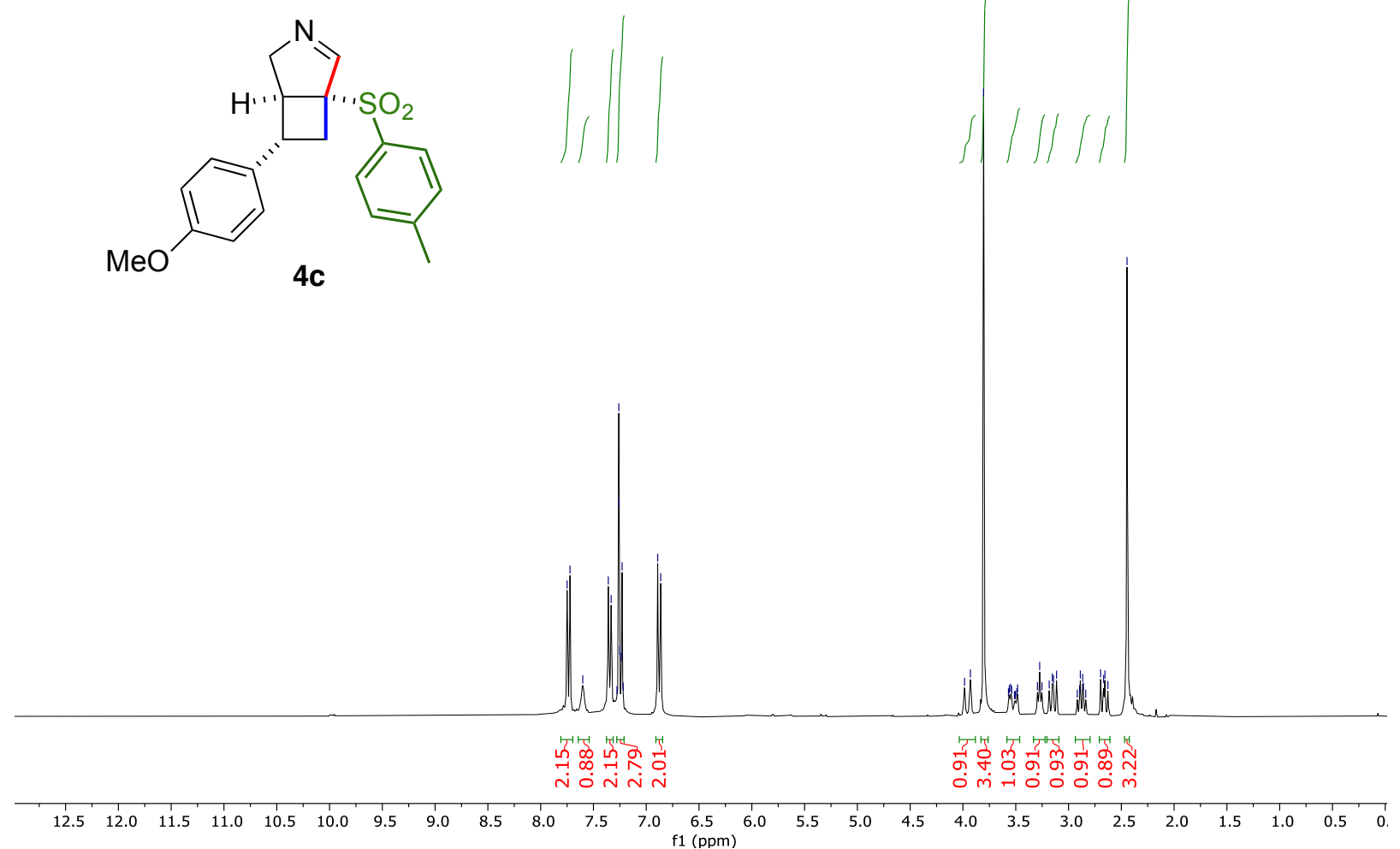

${ }^{13} \mathrm{C}$ NMR (75 $\left.\mathrm{MHz} \mathrm{CDCl}_{3}\right)$

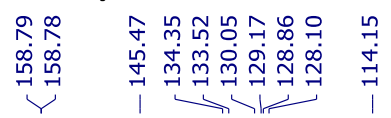

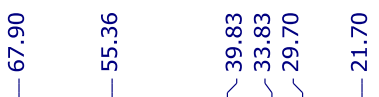

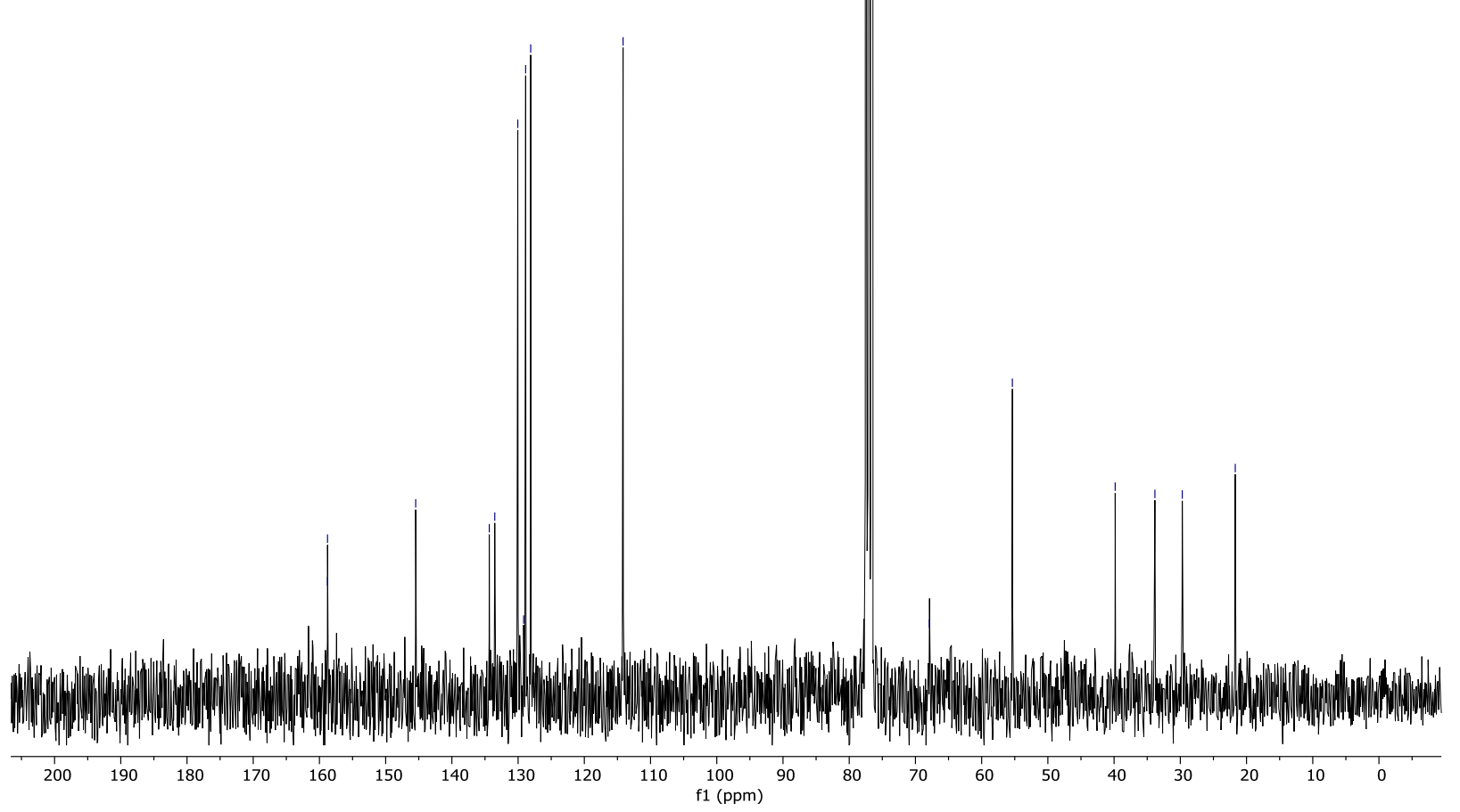


${ }^{1} \mathrm{H}$ NMR (400 MHz, $\mathrm{CDCl}_{3}$ )

AS147.1.fid

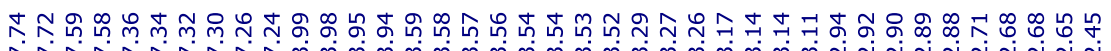

NNヘ<smiles>Cc1ccc(S(=O)(=O)C23C=NC[C@H]2[C@H](c2ccc(Cl)cc2)C3)cc1</smiles>
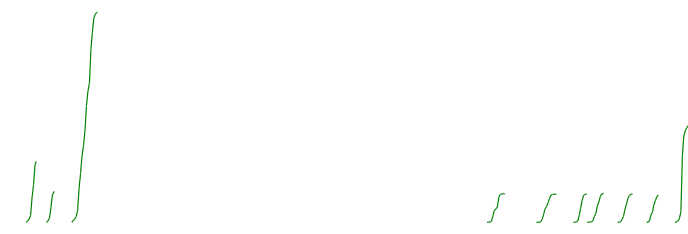

Cl $4 d$

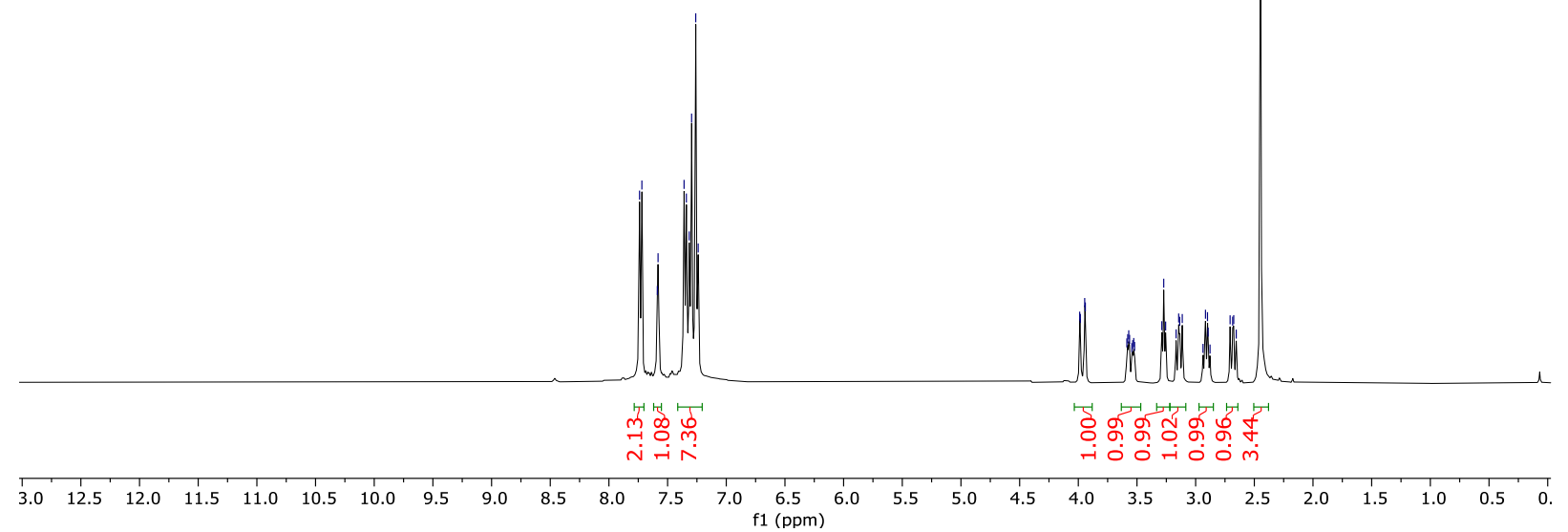

${ }^{13} \mathrm{C}$ NMR (101 MHz, $\left.\mathrm{CDCl}_{3}\right)$

AS147.2.fid

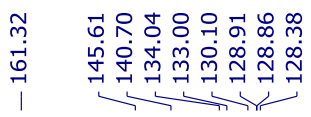

$\mid \begin{array}{ll}0 \\ 0 & 0 \\ 0 & 0 \\ 0 & 0 \\ 1 & 1\end{array}$

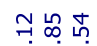

$\dot{\gamma} \dot{m} \stackrel{m}{m}$

$\stackrel{m}{\text { in }}$

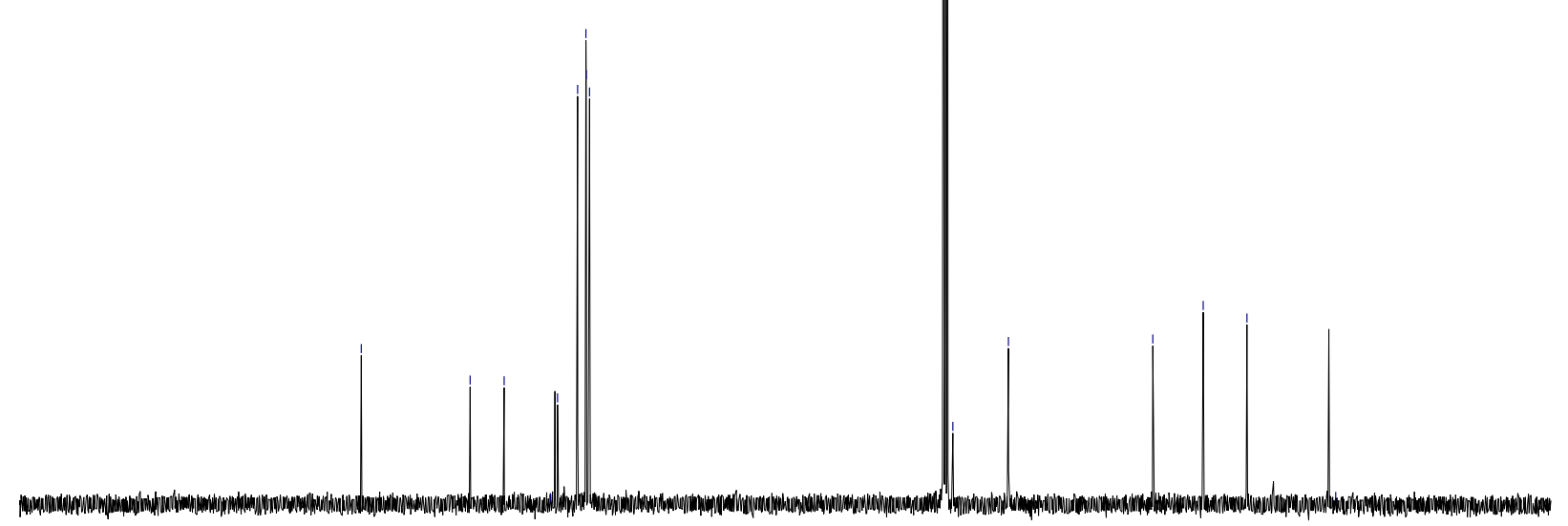

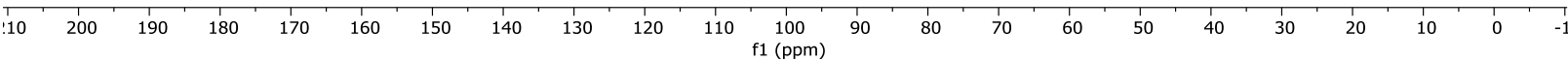


${ }^{1} \mathrm{H}$ NMR (400 MHz, $\left.\mathrm{CDCl}_{3}\right)$

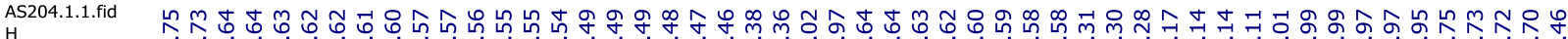

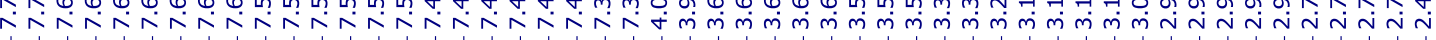<smiles>Cc1ccc([C@]2(O)C=NC[C@H]2c2cccc(C)c2)cc1</smiles>
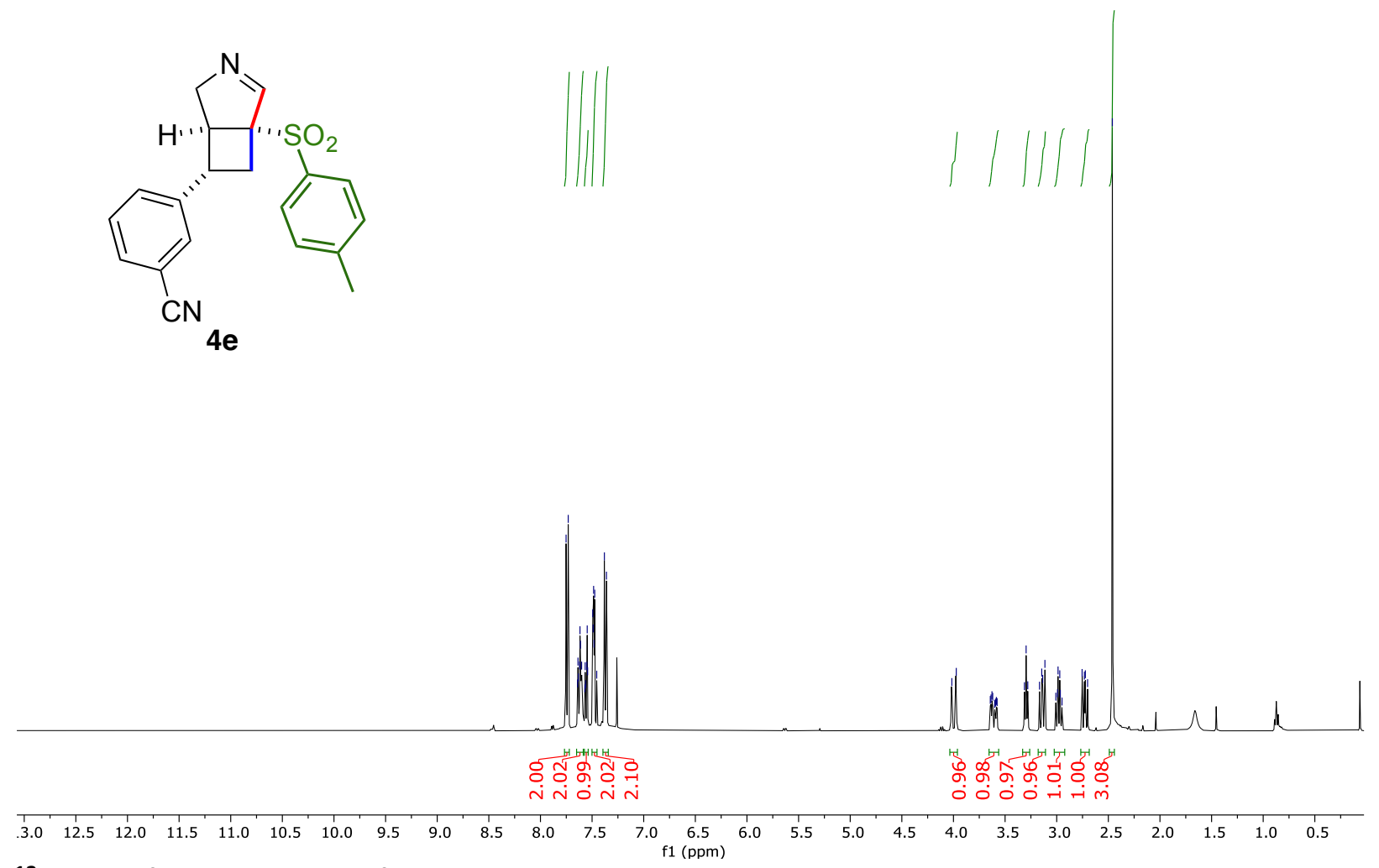

${ }^{13} \mathrm{C}$ NMR (101 MHz, $\left.\mathrm{CDCl}_{3}\right)$

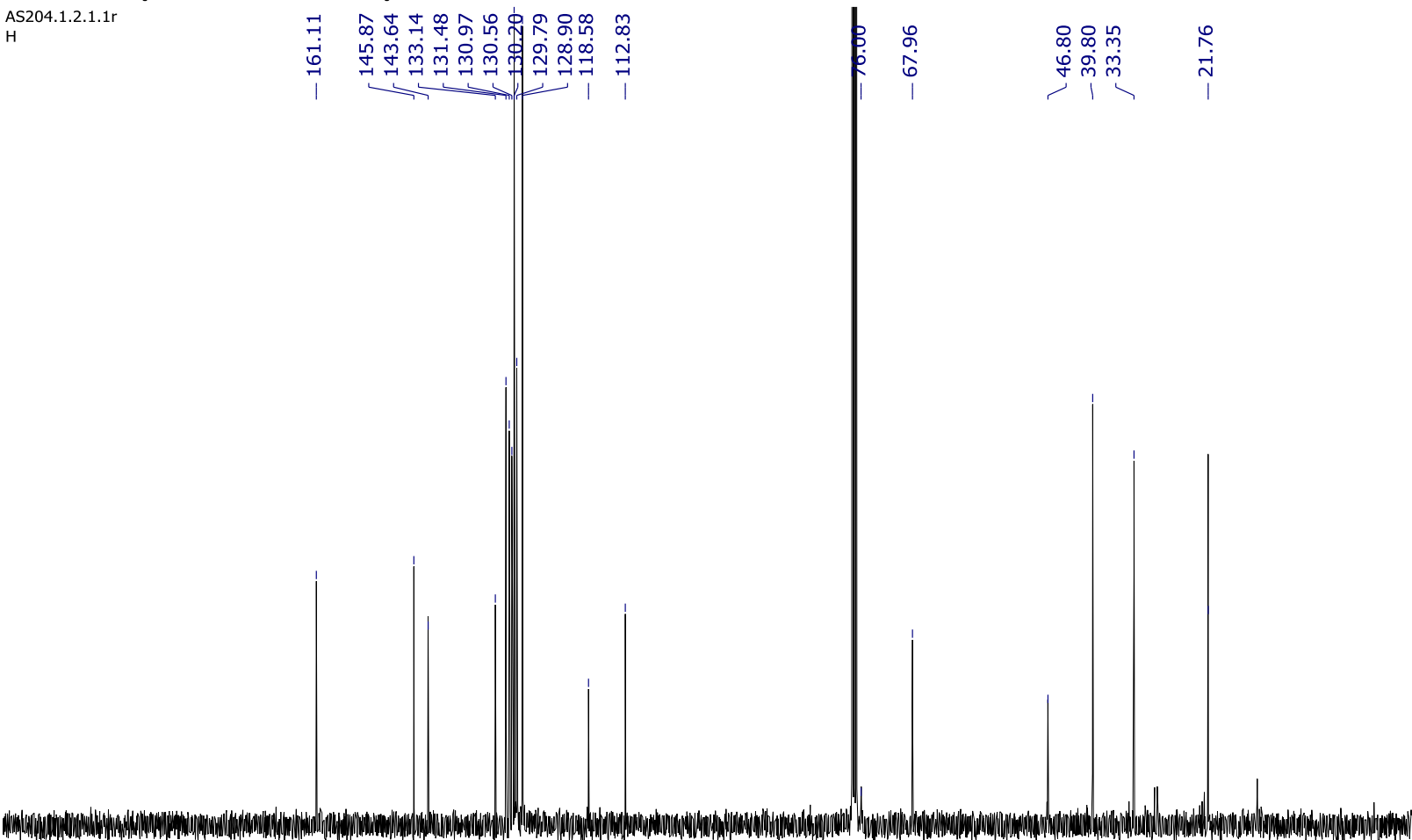

\begin{tabular}{lllllllllllllllllllllll}
\hline 10 & 200 & 190 & 180 & 170 & 160 & 150 & 140 & 130 & 120 & 110 & 100 & 90 & 80 & 70 & 60 & 50 & 40 & 30 & 20 & 10 & 0 &
\end{tabular} 
${ }^{13} \mathrm{C}$ NMR (101 MHz, $\left.\mathrm{CDCl}_{3}\right)$

AS 118.1.1.1

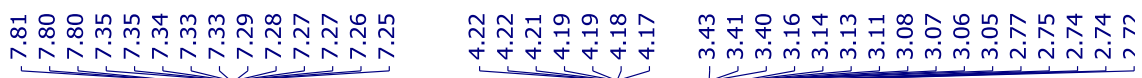

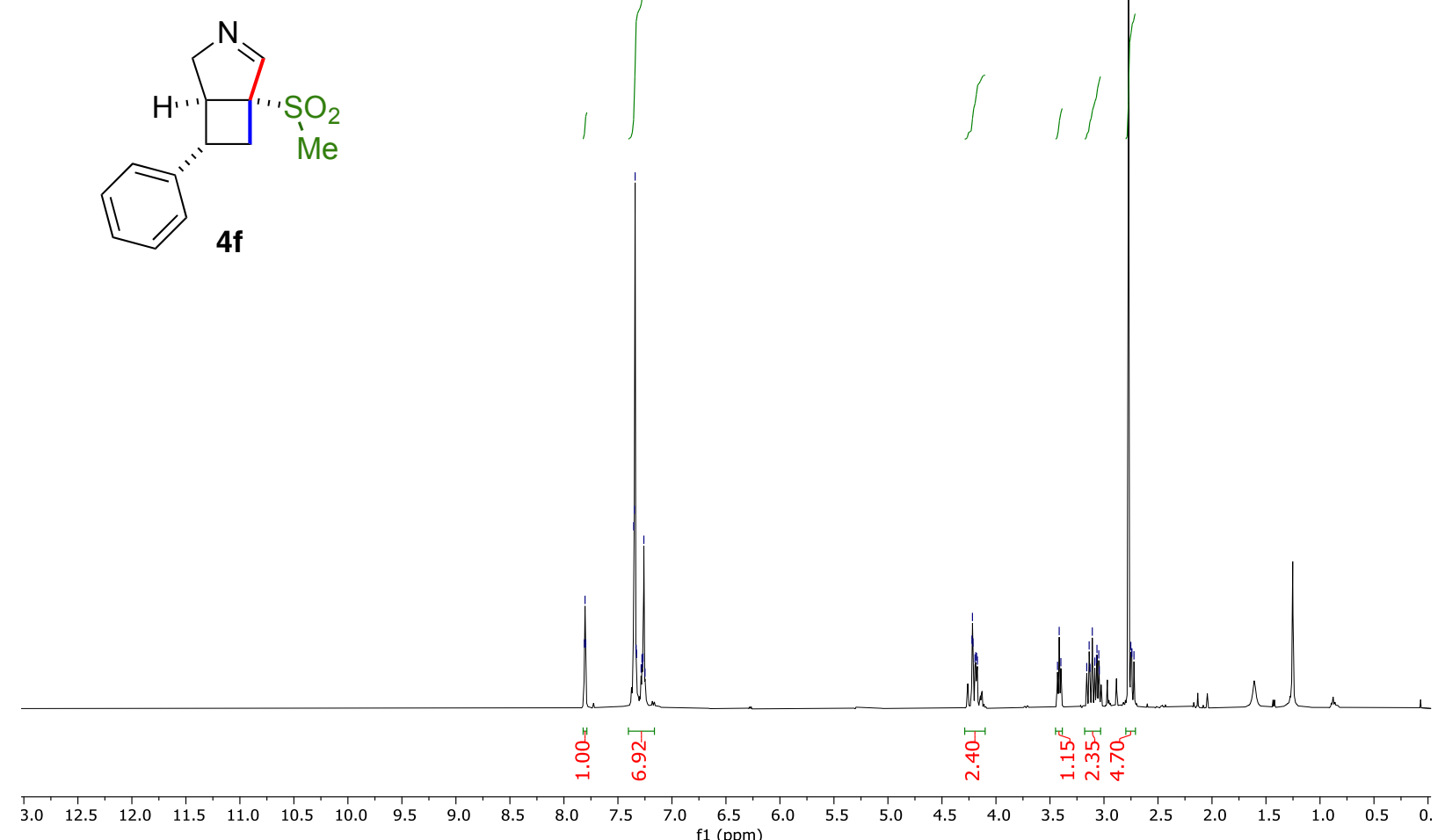

${ }^{13} \mathrm{C}$ NMR (101 MHz, $\mathrm{CDCl}_{3}$ )

AS 118.2.1.1.

$\underset{\substack{\infty \\ 0}}{\infty}$

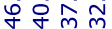

111

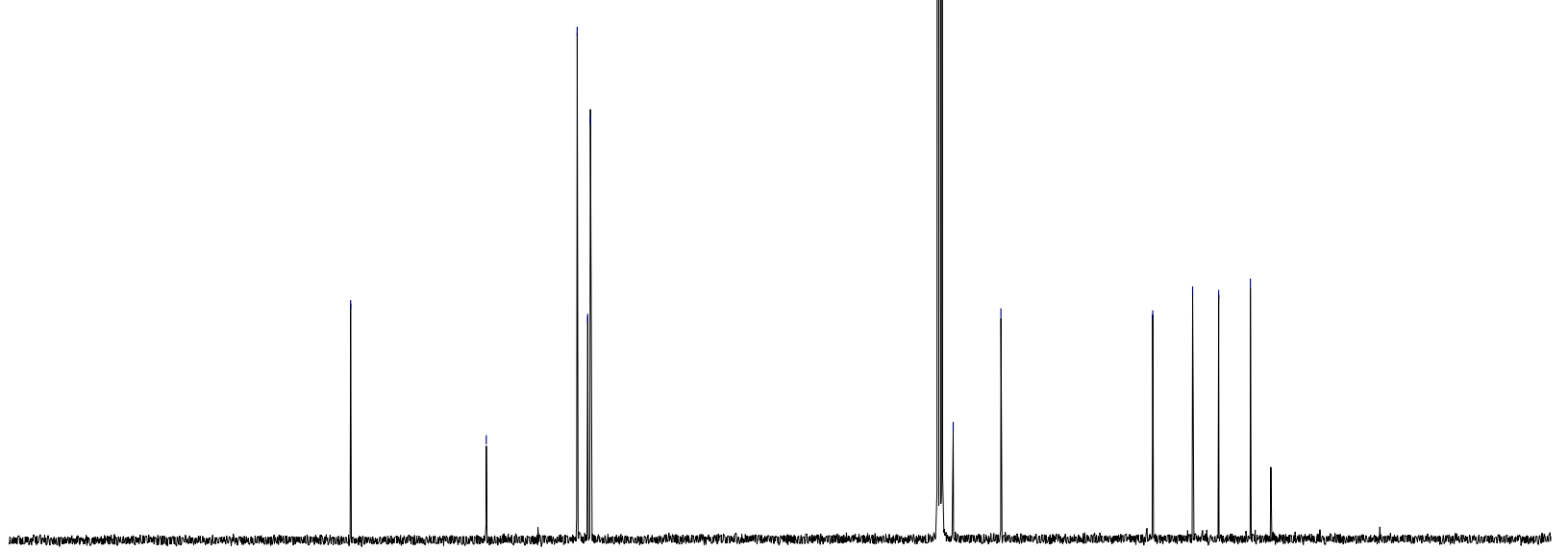

$\begin{array}{lllllllllllllllllllllll}10 & 200 & 190 & 180 & 170 & 160 & 150 & 140 & 130 & 120 & 110 & 100 & 90 & 80 & 70 & 60 & 50 & 40 & 30 & 20 & 10 & 0 & -1\end{array}$ 
${ }^{1} \mathrm{H}$ NMR (400 MHz, $\left.\mathrm{CDCl}_{3}\right)$

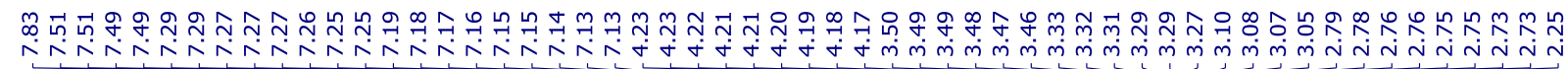<smiles>Cc1ccccc1[C@@H]1C[C@]2(S(C)(=O)=O)C=NC[C@H]12</smiles><smiles>CC=CC#CC(C)C</smiles>
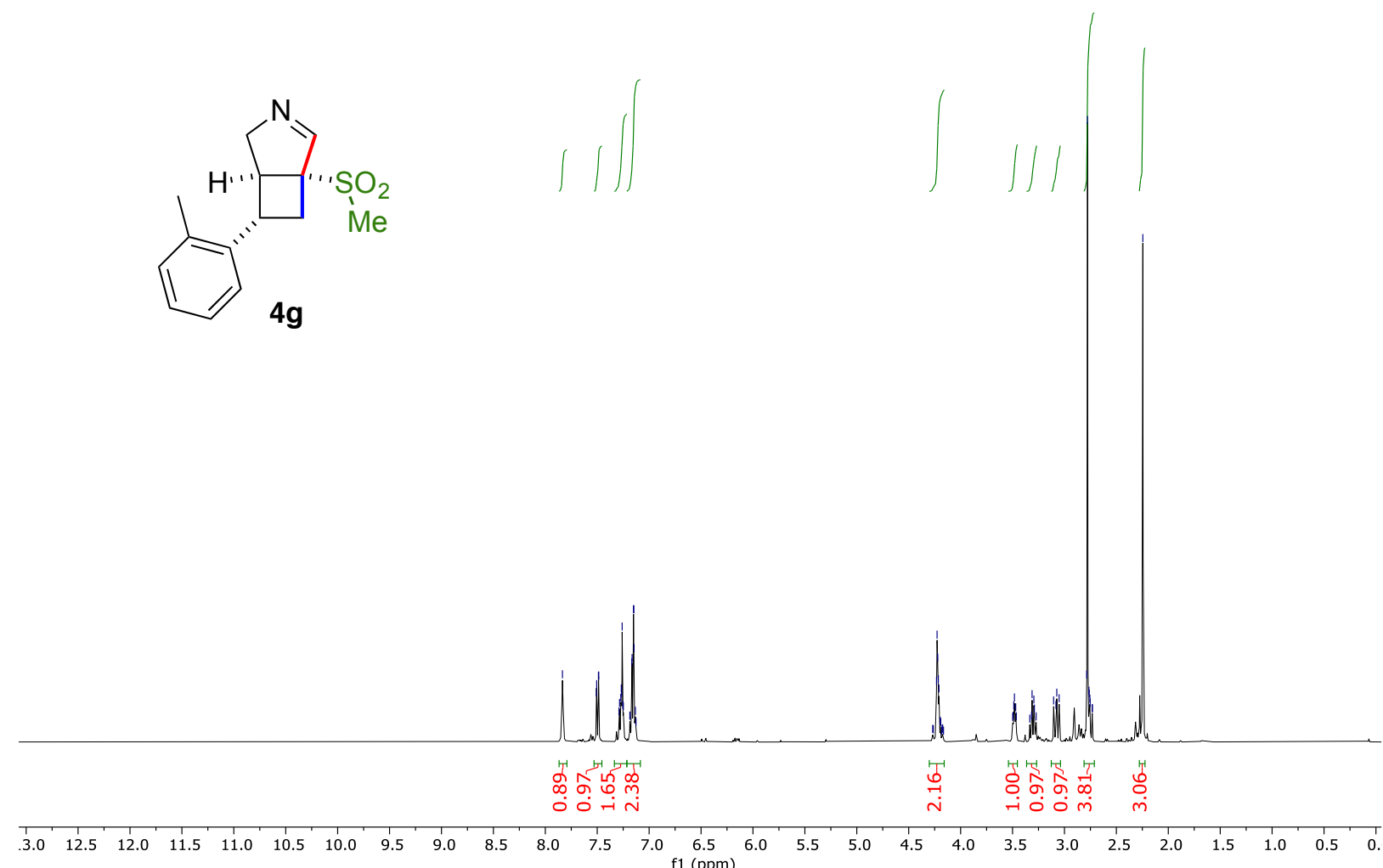

${ }^{13} \mathrm{C}$ NMR (101 MHz, $\mathrm{CDCl}_{3}$ )

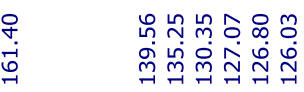

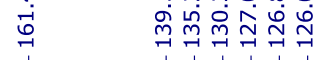

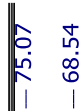

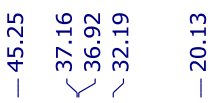

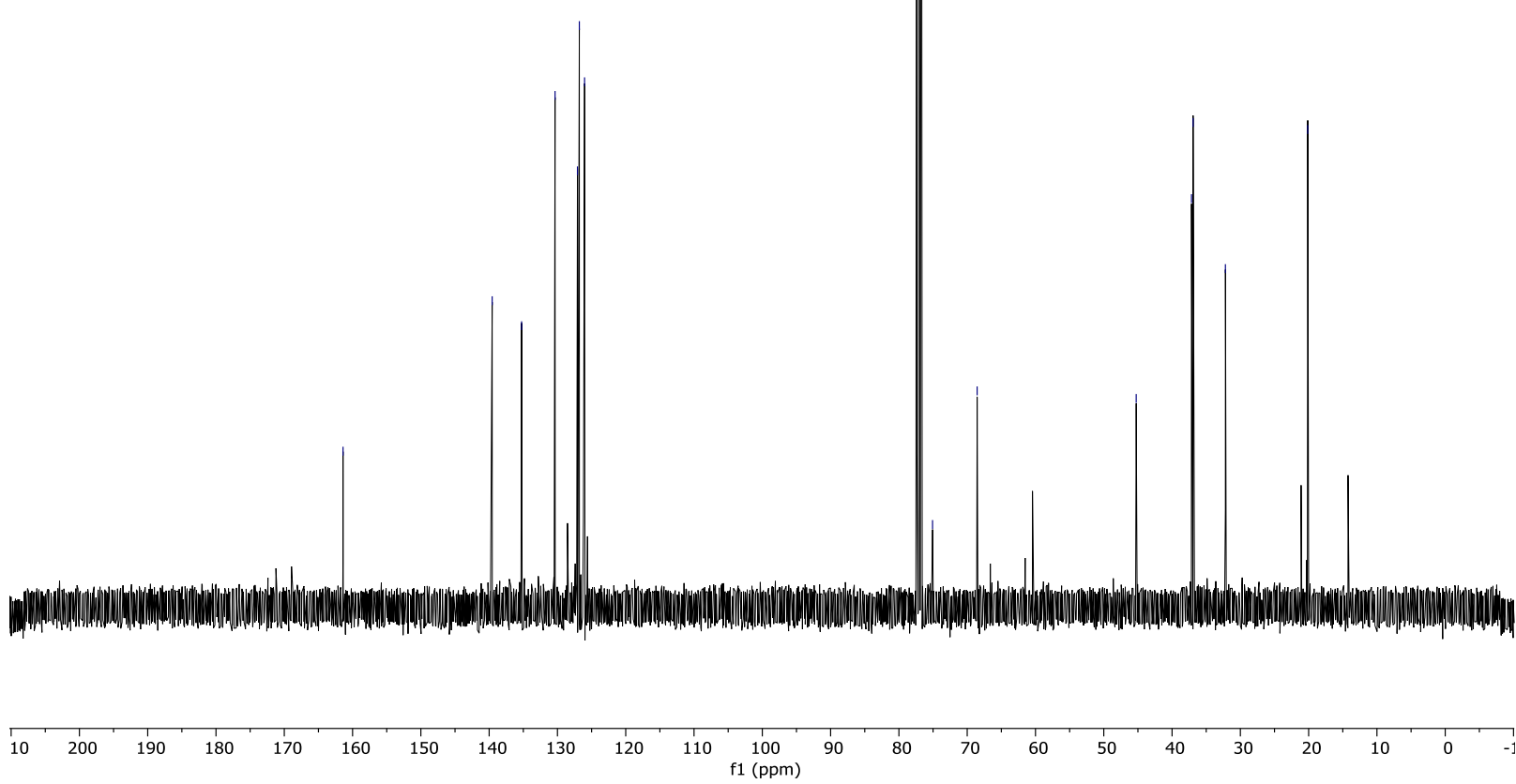




\section{${ }^{1} \mathrm{H}$ NMR (300 MHz, $\left.\mathrm{CDCl}_{3}\right)$}

AS138 H e C.1.fid

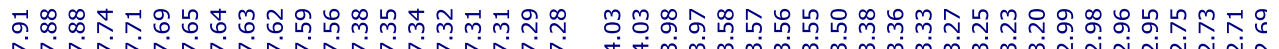

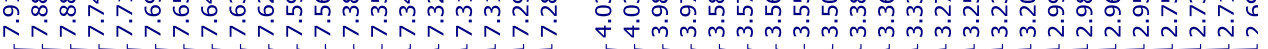<smiles>O=S(=O)(O)[C@]12C=NC[C@H]1[C@@H](c1ccccc1)C2</smiles>
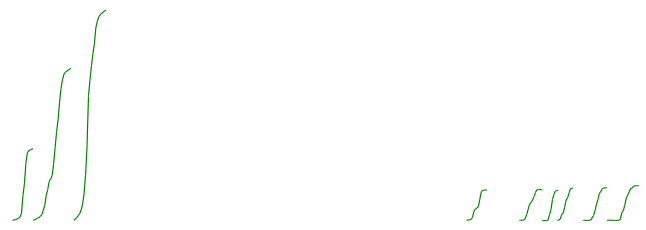

$4 h$

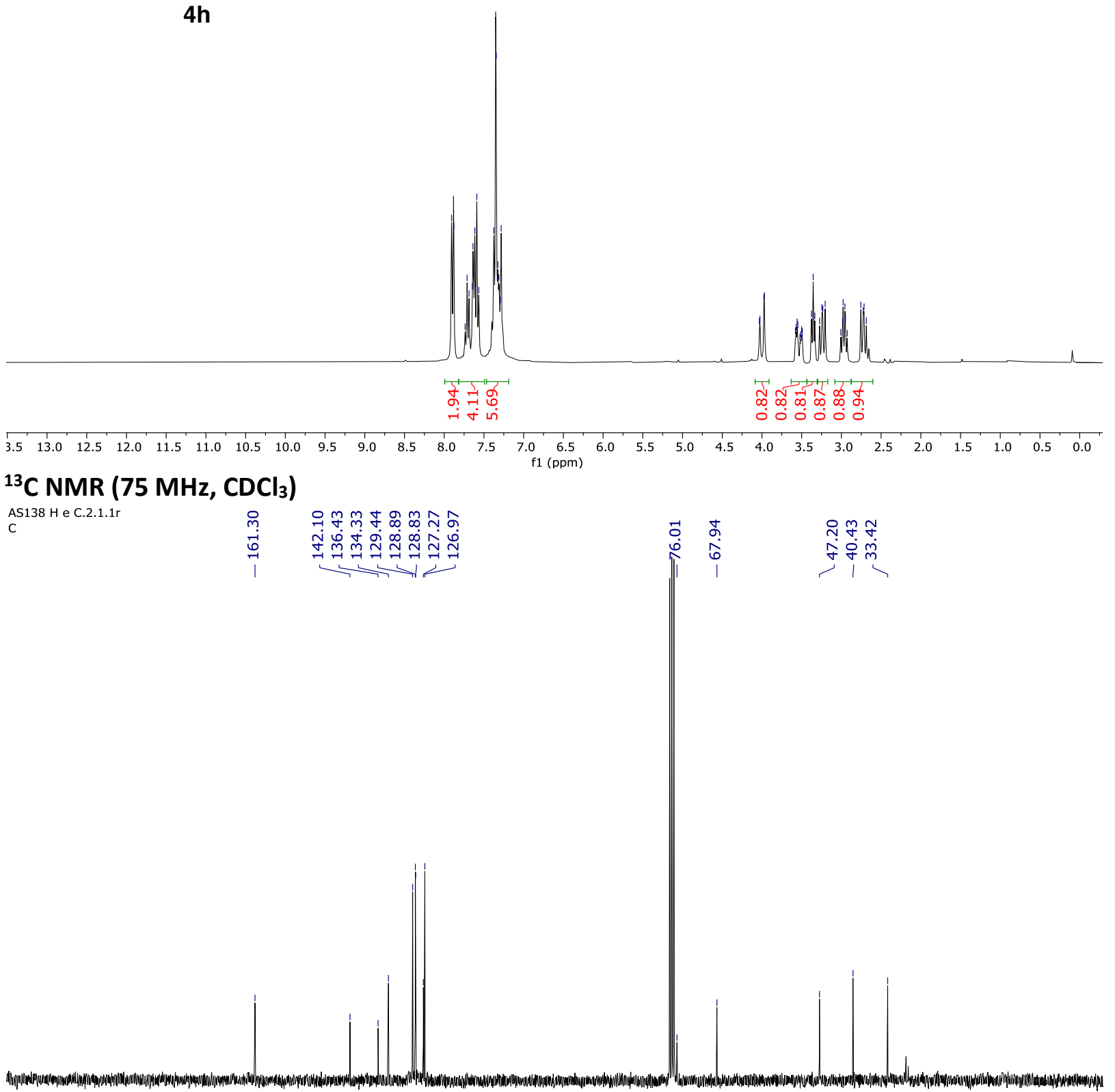

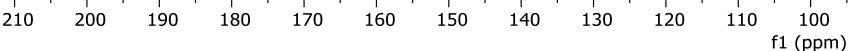


${ }^{1} \mathrm{H}$ NMR (400 MHz, $\mathrm{CDCl}_{3}$ )
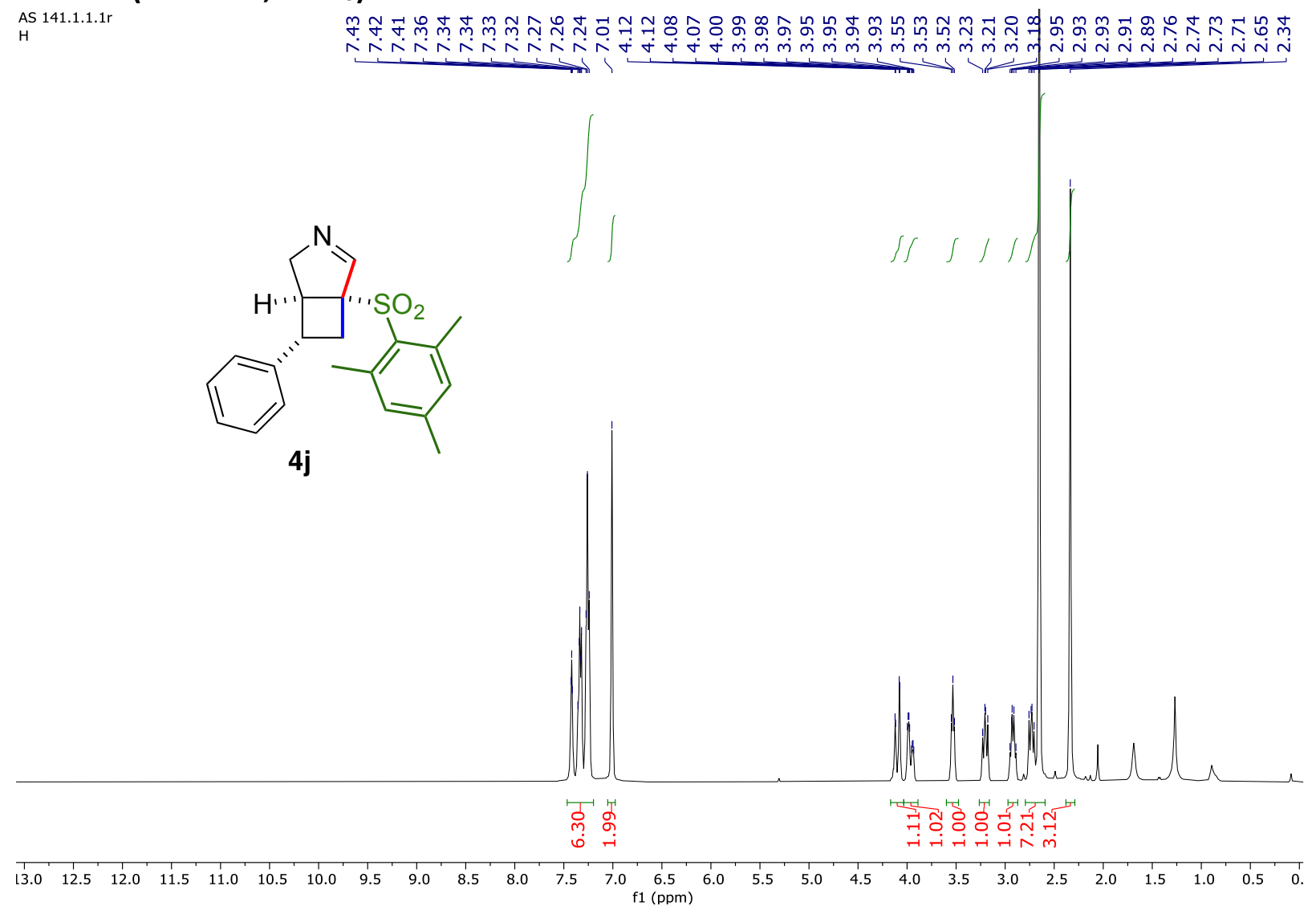

${ }^{13} \mathrm{C}$ NMR (101 $\left.\mathrm{MHz}, \mathrm{CDCl}_{3}\right)$

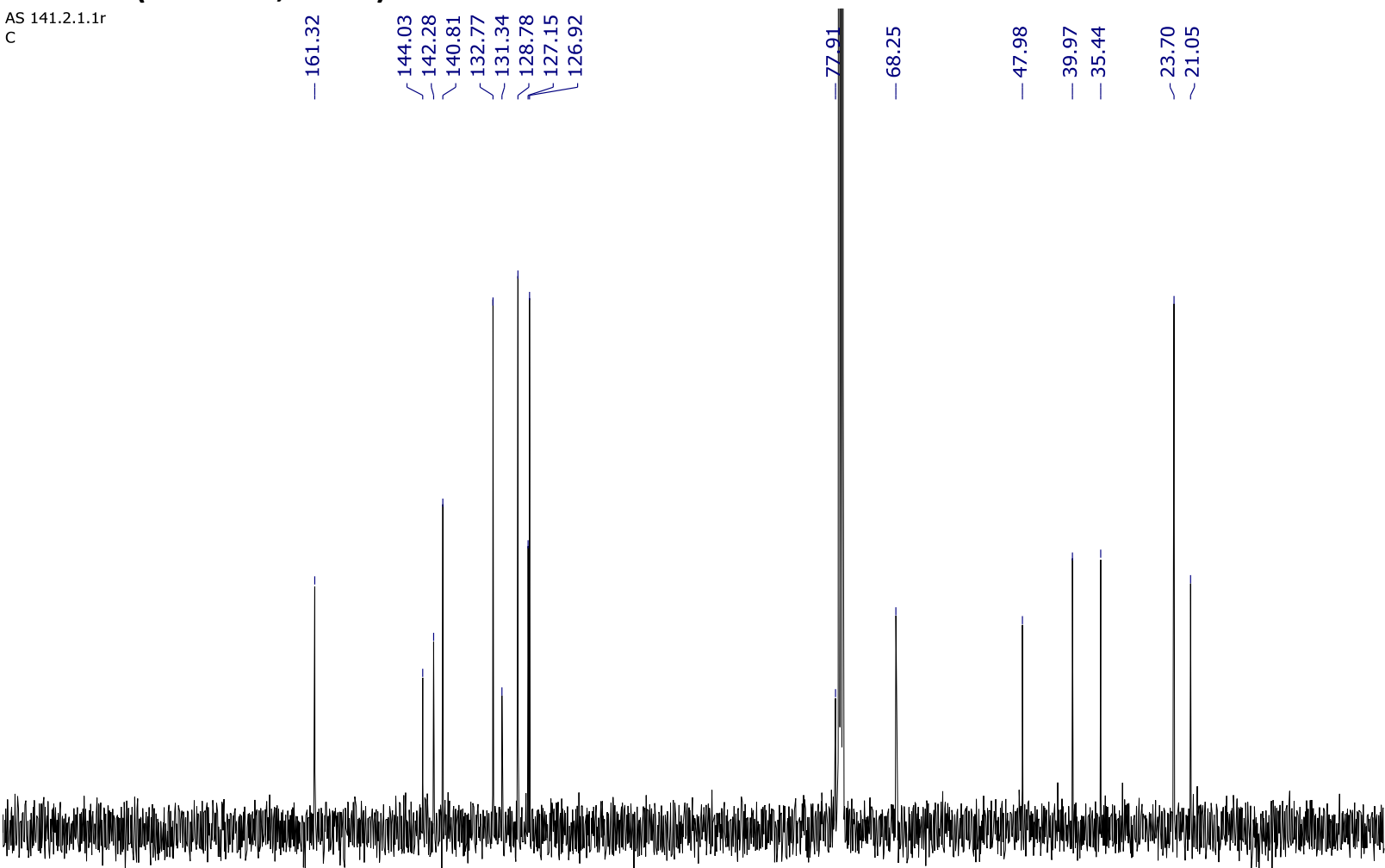

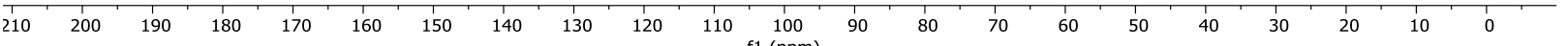


${ }^{1} \mathrm{H}$ NMR $\left(400 \mathrm{MHz}, \mathrm{CDCl}_{3}\right)$

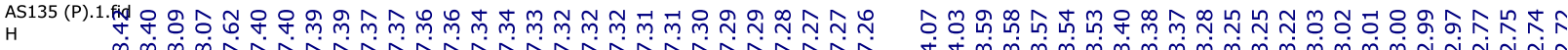

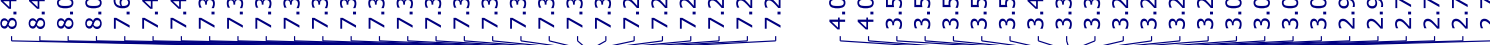<smiles>O=[N+]([O-])c1ccc([C@]2(O)C=NC[C@H]2c2ccccc2)cc1</smiles>
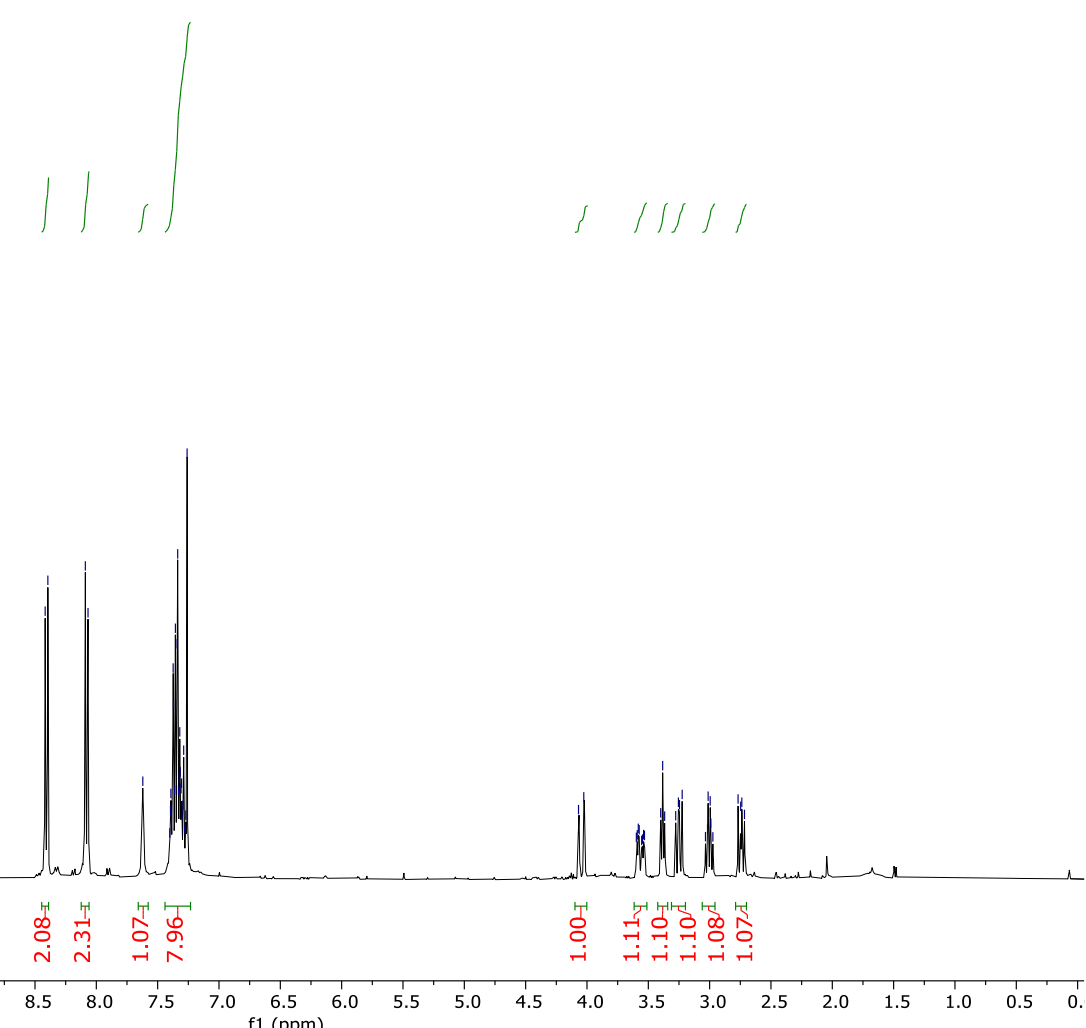

\section{${ }^{13} \mathrm{C}$ NMR (75 MHz, acetone)}

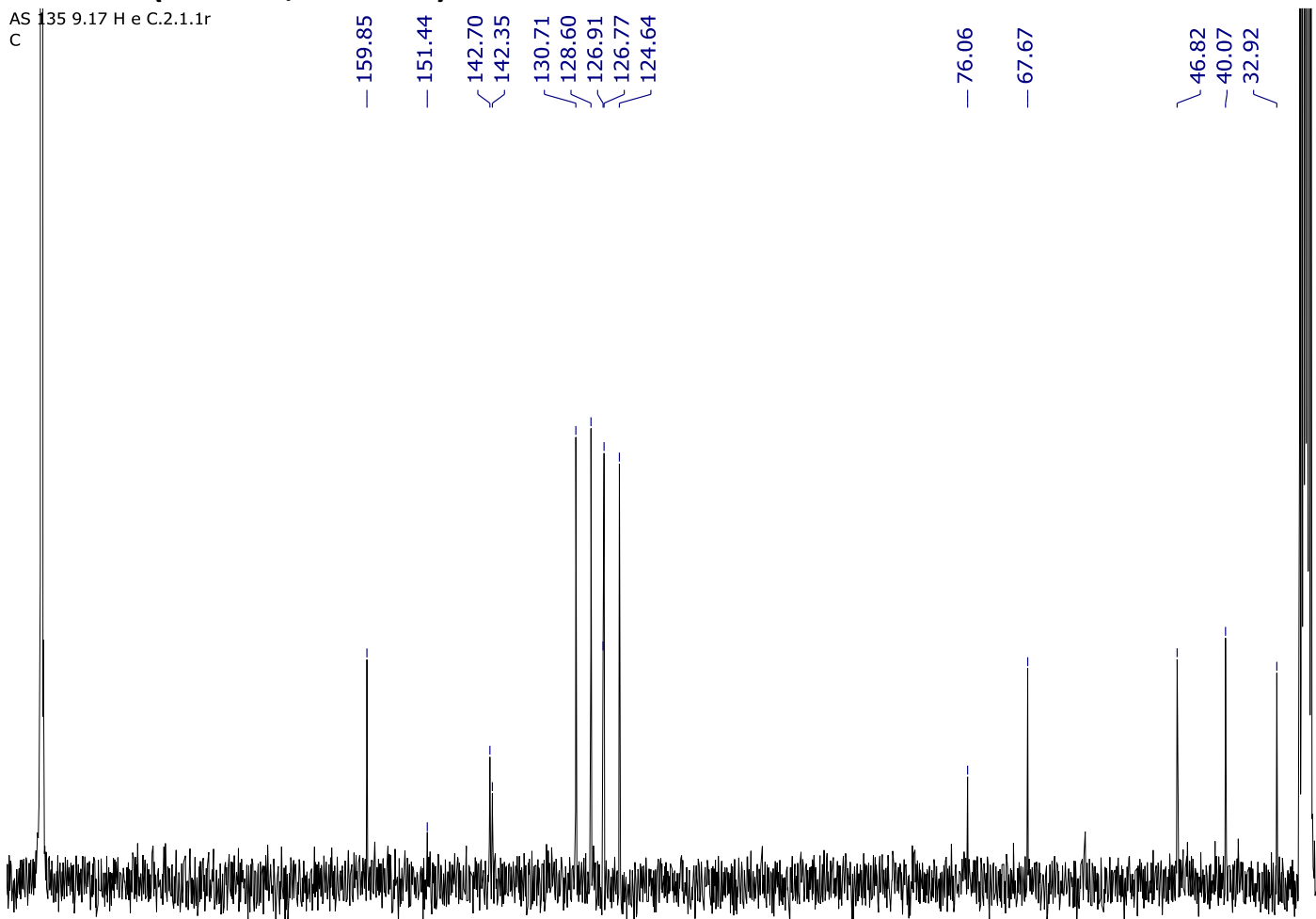

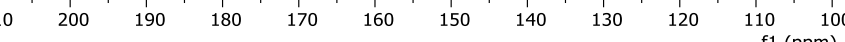


${ }^{1} \mathrm{H}$ NMR (400 MHz, $\left.\mathrm{CDCl}_{3}\right)$

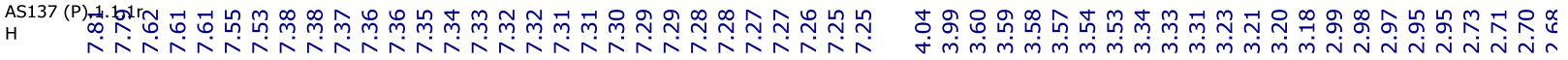
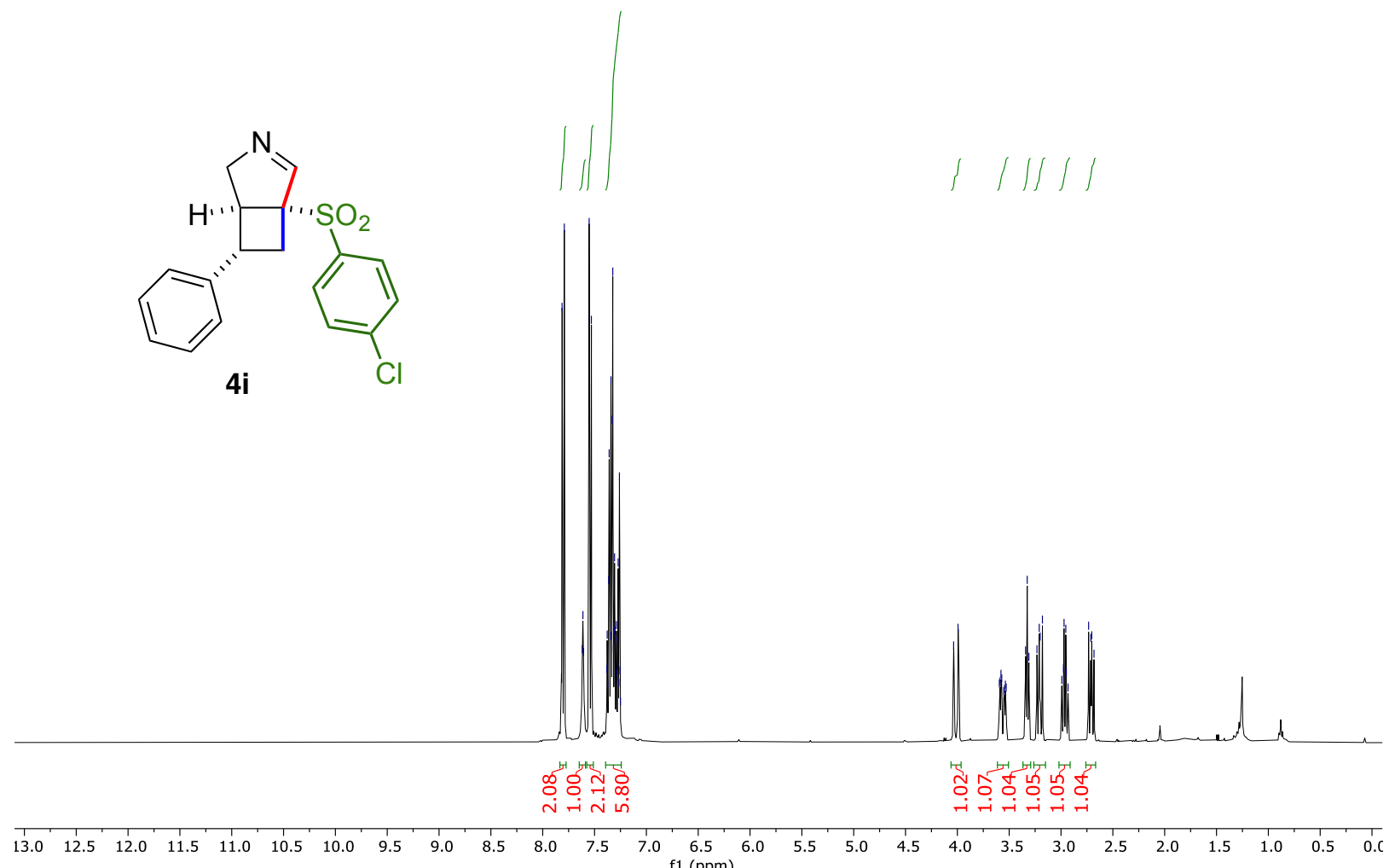

${ }^{13} \mathrm{C} \mathrm{NMR}\left(75 \mathrm{MHz}, \mathrm{CDCl}_{3}\right)$

AS 137.2.1.1

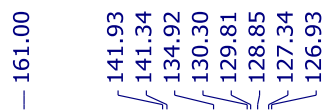

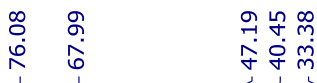

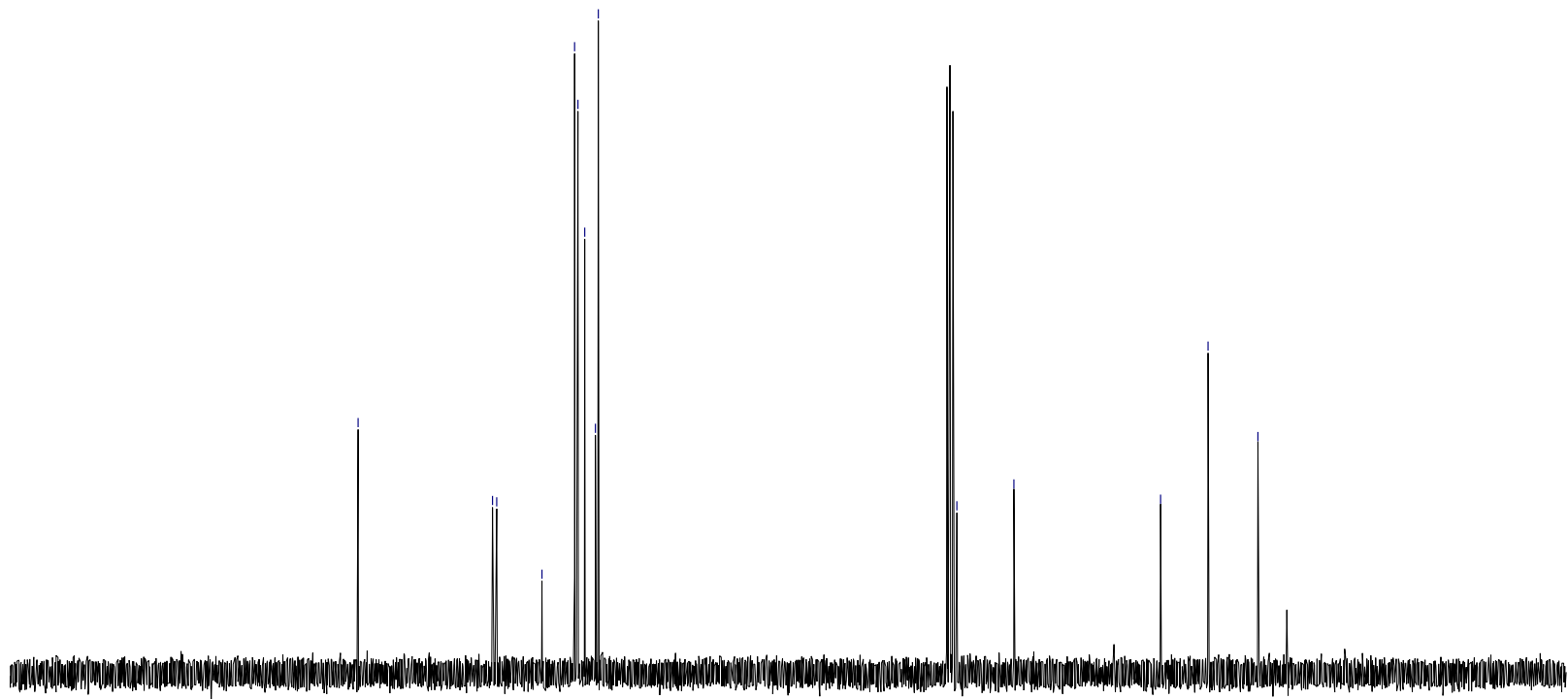

$\begin{array}{llllllllllllllllllllllllllllll}10 & 200 & 190 & 180 & 170 & 160 & 150 & 140 & 130 & 120 & 110 & 100 & 90 & 80 & 70 & 60 & 50 & 40 & 30 & 20 & 10 & 0 & -1\end{array}$ 
${ }^{1} \mathrm{H}$ NMR (300 MHz, $\left.\mathrm{CDCl}_{3}\right)$

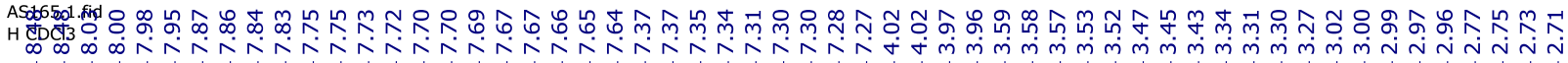<smiles>O=S(=O)(O)c1cccc2ccccc12</smiles>

4!
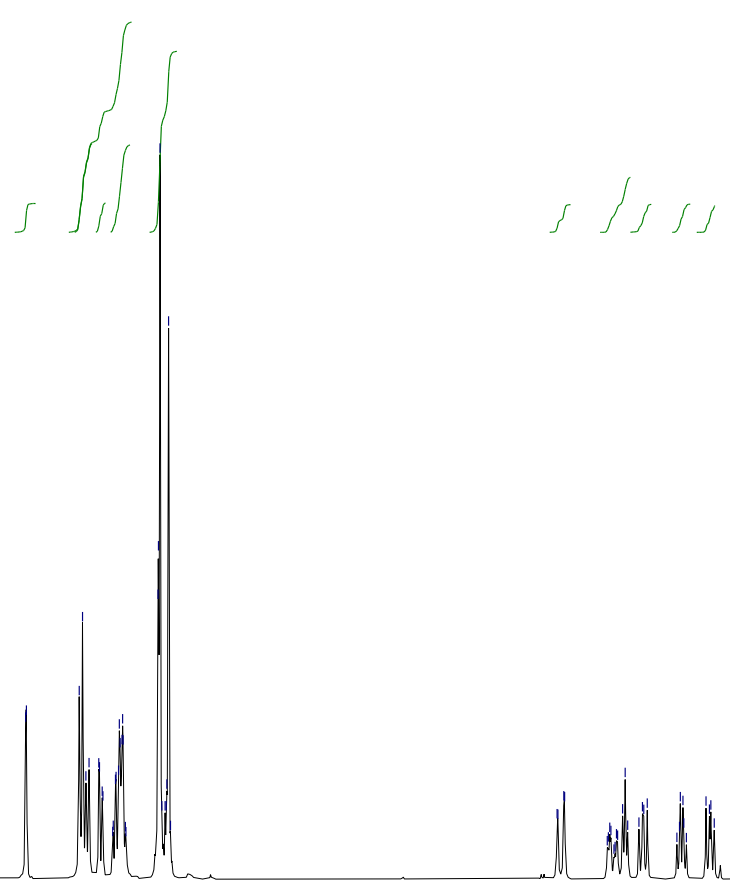

F

का का तर -i

$\begin{array}{lllllllllllllllllllllllllllllll}13.0 & 12.5 & 12.0 & 11.5 & 11.0 & 10.5 & 10.0 & 9.5 & 9.0 & 8.5 & 8.0 & 7.5 & 7.0 & 6.5 & 6.0 & 5.5 & 5.0 & 4.5 & 4.0 & 3.5 & 3.0 & 2.5 & 2.0 & 1.5 & 1.0 & 0.5 & 0.0\end{array}$ ${ }^{13} \mathrm{C}$ NMR (75 MHz, $\mathrm{CDCl}_{3}$ )

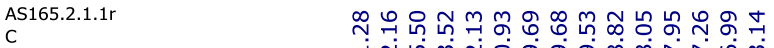

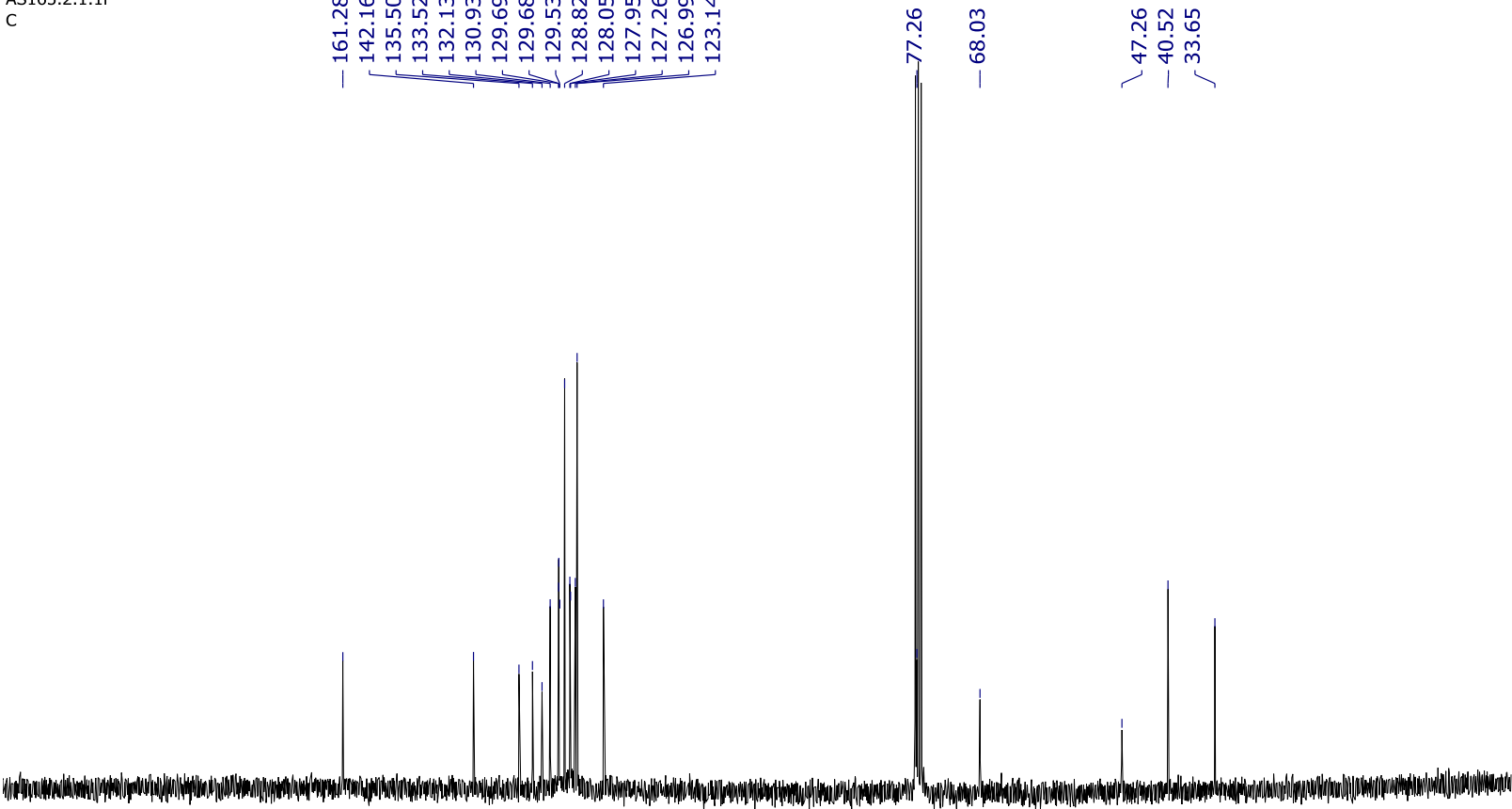

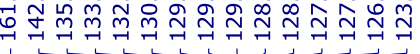

ำ ำ 
${ }^{1} \mathrm{H}$ NMR $\left(400 \mathrm{MHz}, \mathrm{CDCl}_{3}\right)$

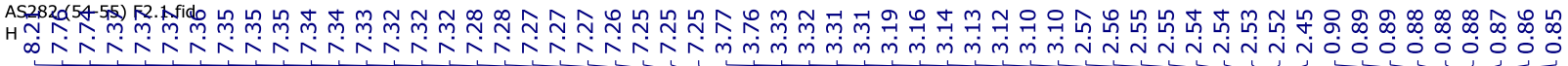
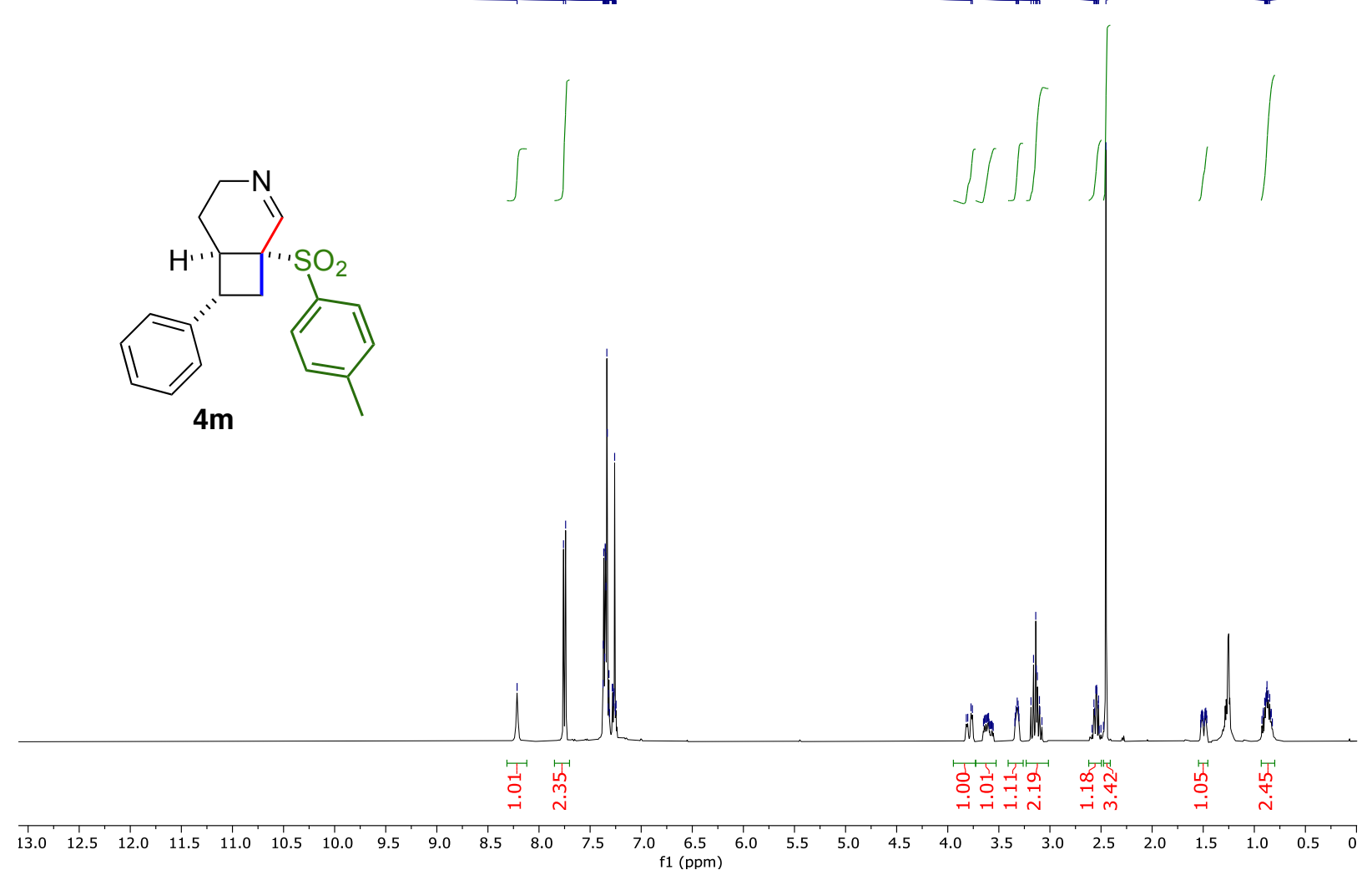

${ }^{13} \mathrm{C}$ NMR (101 $\left.\mathrm{MHz}, \mathrm{CDCl}_{3}\right)$

\section{AS282 (54-55) F2.2.1.1} AS

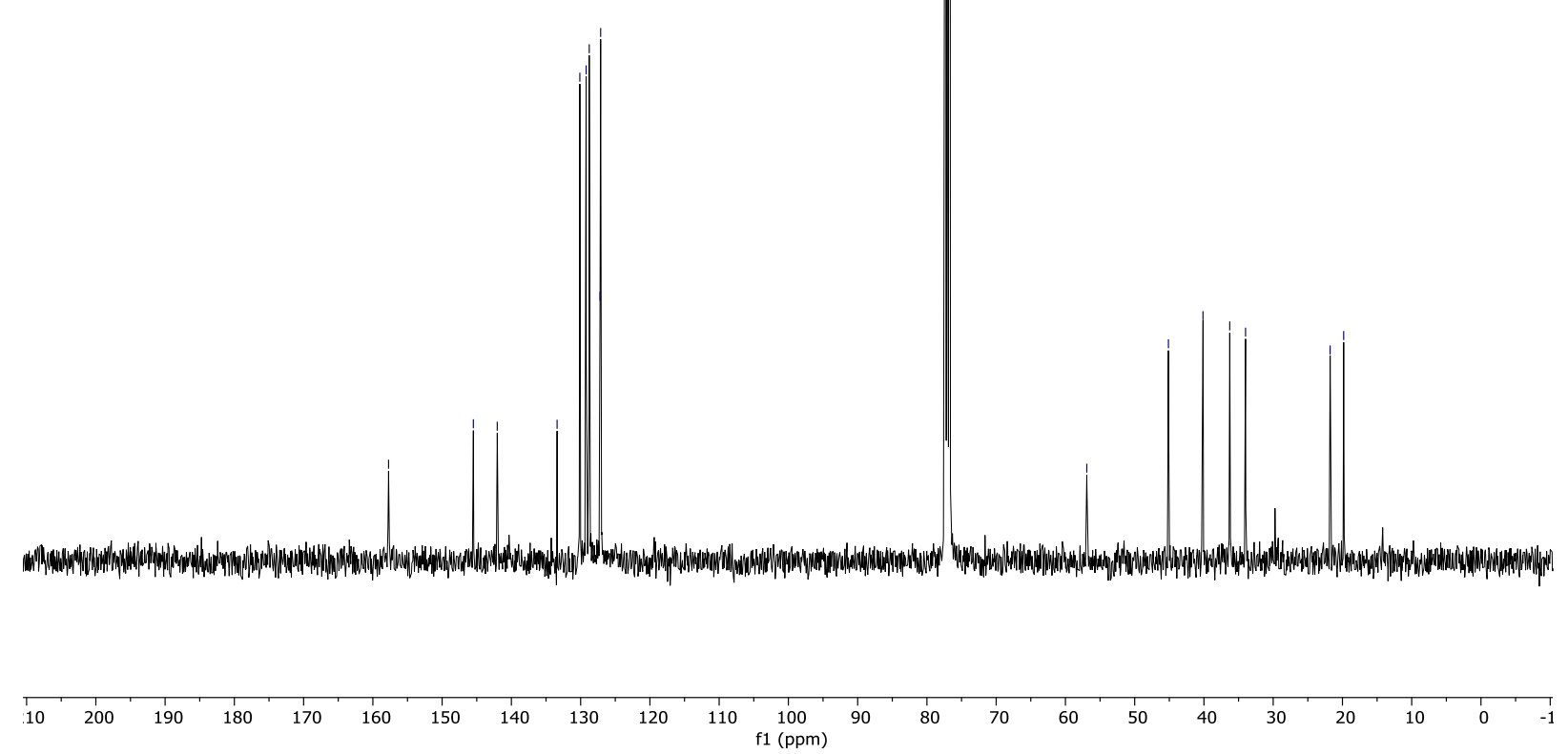




\section{Xyz coordinates}

optimized at the M06/Def2-tzvp level with DMF as implicit solvent

\section{1}

35

scf done: -1146.775960

$\begin{array}{llll}\text { C } 8.646922 & -4.891170 & 0.108052\end{array}$

$\begin{array}{llll}\text { C } & 8.872387 & -3.781292 & 0.911007\end{array}$

$\begin{array}{llll}\text { C } & 7.965534 & -2.740312 & 0.934692\end{array}$

$\begin{array}{llll}\text { C } & 6.809457 & -2.780705 & 0.152718\end{array}$

$\begin{array}{llll}\text { C } & 6.596896 & -3.903239 & -0.646698\end{array}$

C $7.504404 \quad-4.948094 \quad-0.671270$

$\begin{array}{llll}\text { C } & 5.820225 & -1.706686 & 0.138601\end{array}$

$\begin{array}{llll}\text { C } & 5.871368 & -0.552054 & 0.795402\end{array}$

C $4.794801 \quad 0.472816 \quad 0.728424$

$\begin{array}{lllll}\mathrm{N} & 5.300042 & 1.770729 & 0.266302\end{array}$

$\begin{array}{llll}\text { S } & 4.930387 & 2.261069 & -1.240015\end{array}$

$\begin{array}{lllll}\text { O } & 5.695036 & 3.443515 & -1.507423\end{array}$

$\begin{array}{llll}\text { C } & 5.756403 & 2.715200 & 1.285288\end{array}$

$\begin{array}{llll}\text { C } & 4.636625 & 3.374071 & 2.037801\end{array}$

$\begin{array}{llll}\text { C } & 4.318008 & 3.069756 & 3.261347\end{array}$

$\begin{array}{llll}\text { C } & 3.226193 & 2.713589 & -1.218101\end{array}$

\begin{tabular}{lllll} 
O & 5.048949 & 1.125125 & -2.106175 \\
\hline & 4.342688 & 0.635277 & 1.714684
\end{tabular}

$\begin{array}{cccc}\text { H } & 4.342688 & 0.635277 & 1.714684\end{array}$

$\begin{array}{llll}H & 3.991751 & 0.128284 & 0.072813\end{array}$

$\begin{array}{llll}\text { H } & 2.943782 & 2.977334 & -2.236785\end{array}$

$\begin{array}{lllll}H & 2.633488 & 1.865798 & -0.876983\end{array}$

$\begin{array}{lllll}H & 3.090074 & 3.566963 & -0.556786\end{array}$

$\begin{array}{llll}H & 6.723965 & -0.305540 & 1.425004\end{array}$

$\begin{array}{llll}\text { H } & 4.949727 & -1.892269 & -0.490726\end{array}$

H $\quad \begin{array}{llll}5.701638 & -3.949387 & -1.258691\end{array}$

H $7.317176-5.809676 \quad-1.301561$

H $\quad 9.359901 \quad-5.7070550 .093443$

$\begin{array}{llll}H & 9.763328 & -3.730912 & 1.526339\end{array}$

$\begin{array}{llll}\text { H } & 8.156937 & -1.884856 & 1.572945\end{array}$

H $\quad 6.383985 \quad 3.461102 \quad 0.798280$

$\begin{array}{llll}H & 6.393669 & 2.157148 & 1.975867\end{array}$

$\begin{array}{llll}\text { H } & 4.069650 & 4.143559 & 1.516806\end{array}$

$\begin{array}{llll}\text { C } & 4.022311 & 2.761223 & 4.485600\end{array}$

$\begin{array}{lllll}\text { H } & 4.473126 & 3.280904 & 5.324924\end{array}$

H $\quad 3.310502 \quad 1.974942 \quad 4.715211$

${ }^{3} 1$

35

scf done: -1146.695914

\begin{tabular}{|c|c|c|c|}
\hline & & & \\
\hline & 1.646817 & 0.020474 & \\
\hline & 1.169530 & & \\
\hline & -0.175502 & 1330 & \\
\hline & -1.097 & & \\
\hline & -0.650 & -0.4 & \\
\hline & & & \\
\hline & 3.600 & -0.34 & \\
\hline & 3.630 & & \\
\hline & 4.167 & & \\
\hline & 5.419396 & 1.965878 & 0.27 \\
\hline & 3.211 & & \\
\hline & 4.0672 & 4.352 & -0.7 \\
\hline & 2.4075 & 3.354649 & 0.997 \\
\hline & & & \\
\hline & & & \\
\hline
\end{tabular}




$\begin{array}{rrrr}H & 4.235109 & 0.093384 & -1.546488 \\ H & 1.873976 & 4.304512 & 1.007939 \\ H & 1.712397 & 2.524328 & 1.117405 \\ H & 3.161139 & 3.336632 & 1.786083 \\ H & 3.687110 & 0.426440 & 2.489226 \\ H & 3.930409 & -1.370324 & 0.292167 \\ H & 1.888113 & 0.848597 & 3.973887 \\ H & -0.519579 & 0.853987 & 4.494541 \\ H & -2.155957 & 0.036516 & 2.824950 \\ H & -1.365082 & -0.800438 & 0.634739 \\ H & 1.037014 & -0.820088 & 0.105723 \\ H & 5.696559 & 3.018254 & 0.344785 \\ H & 5.284126 & 1.582453 & 1.290368 \\ \text { C } & 6.503765 & 1.194740 & -0.414802 \\ \text { C } & 7.028755 & 0.117472 & 0.089662 \\ \text { H } & 6.833710 & 1.559425 & -1.385159 \\ \text { C } & 7.536242 & -0.954095 & 0.614935 \\ H & 8.401278 & -0.912563 & 1.268913 \\ \text { H } & 7.114436 & -1.933904 & 0.413342\end{array}$

TS (1-II)

35

scf done: -1146.690499

$\begin{array}{llll}\text { C } & 0.207718 & 0.207421 & 0.045621\end{array}$

C $\quad 0.227805 \quad 0.362878 \quad 1.412456$

$\begin{array}{llll}\text { C } & 1.428893 & 0.283727 & 2.111594\end{array}$

$\begin{array}{llll}\text { C } & 2.610199 & 0.041784 & 1.416536\end{array}$

C $2.601844 \quad-0.115298 \quad 0.048653$

C $1.395220 \quad-0.032838-0.684962$

$\begin{array}{llll}\text { C } & 1.334521 & -0.209032 & -2.078273\end{array}$

$\begin{array}{llll}\text { C } & 2.464976 & -0.491535 & -2.947029\end{array}$

$\begin{array}{llll}\text { C } & 2.211171 & -0.374917 & -4.430183\end{array}$

N $1.799636 \quad-1.689472 \quad-4.909082$

S $\quad 0.397628 \quad-1.971286 \quad-5.671601$

C $0.738733 \quad-1.657369-7.368876$

C $2.675146 \quad-2.708597 \quad-4.358200$

$\begin{array}{llll}\text { C } & 2.758946 & -2.451430 & -2.862914\end{array}$

C $1.754612-3.064111-2.091200$

C $\quad 0.700574 \quad-3.319107 \quad-1.382169$

$\begin{array}{llll}\text { O } & -0.560913 & -0.990215 & -5.249559\end{array}$

$\begin{array}{llll}\text { O } & 0.092205 & -3.364481 & -5.526029\end{array}$

H $\quad 3.123914 \quad-0.076704 \quad-4.956463$

H $1.432030 \quad 0.355115-4.657623$

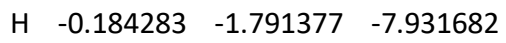

H $1.092212 \quad-0.630957 \quad-7.463912$

H $\quad \begin{array}{llll}\text { H.499997 } & -2.357147 & -7.709023\end{array}$

H $3.417495 \quad-0.074907 \quad-2.624133$

H $\quad 0.349644 \quad-0.184250 \quad-2.542114$

$\begin{array}{llll}H & -0.730269 & 0.269418 & -0.497870\end{array}$

$\begin{array}{llll}H & -0.697562 & 0.547651 & 1.946273\end{array}$

H $1.444061 \quad 0.405138 \quad 3.188009$

H $3.547643 \quad-0.0274851 .956896$

H $3.533286-0.312562 \quad-0.470726$

$\begin{array}{llll}\text { H } & 3.668497 & -2.610637 & -4.808526\end{array}$

H $\quad 2.285211-3.698671 \quad-4.588063$

H $3.760069-2.544180 \quad-2.442046$

H $0.425948 \quad-4.337785-1.102552$

H $\quad 0.027601-2.540592-1.009330$

${ }^{3}$ II

35

scf done: -1146.721976

C $0.000000 \quad 0.000000 \quad 0.000000$ 


\begin{tabular}{lrrr} 
C & 0.000000 & 0.000000 & 1.552151 \\
C & 1.493934 & 0.000000 & 1.841920 \\
N & 2.044117 & 0.860602 & 0.780400 \\
C & 1.088770 & 1.025926 & -0.322296 \\
C & -0.681856 & 1.185584 & 2.135069 \\
C & -2.042366 & 1.251295 & 2.482729 \\
C & -2.558302 & 2.426394 & 3.079572 \\
C & -3.881887 & 2.523519 & 3.441686 \\
C & -4.750603 & 1.458622 & 3.223894 \\
C & -4.270096 & 0.292918 & 2.634503 \\
C & -2.947691 & 0.184359 & 2.268531 \\
S & 3.638987 & 0.824460 & 0.468057 \\
O & 3.920256 & 1.889050 & -0.448340 \\
C & -1.288492 & 0.276039 & -0.620527 \\
C & -1.819900 & 1.017705 & -1.548901 \\
C & 3.938107 & -0.698252 & -0.368775 \\
O & 4.311241 & 0.769843 & 1.732058 \\
H & 1.896194 & -1.018212 & 1.779023 \\
H & 1.734284 & 0.403290 & 2.825976 \\
H & 5.003891 & -0.750560 & -0.587141 \\
H & 3.651252 & -1.526536 & 0.277966 \\
H & 3.368766 & -0.717407 & -1.297434 \\
H & -0.457238 & -0.923492 & 1.916615 \\
H & -0.076342 & 2.071671 & 2.315687 \\
H & -1.882349 & 3.257805 & 3.252245 \\
H & -4.248751 & 3.435484 & 3.898863 \\
H & -5.792995 & 1.536256 & 3.508772 \\
H & -4.943810 & -0.537884 & 2.458097 \\
H & -2.598155 & -0.728078 & 1.796698 \\
H & 1.550913 & 0.854231 & -1.296332 \\
H & 0.678382 & 2.040833 & -0.319920 \\
H & 0.354389 & -0.990611 & -0.317258 \\
H & -2.885355 & 1.008935 & -1.764616 \\
H & -1.215908 & 1.687196 & -2.168250 \\
& & & \\
\hline
\end{tabular}

\section{TS (1-II')}

35

scf done: -1146.678467

$\begin{array}{lccc}\text { C } & 0.045452 & 0.130642 & 0.336764 \\ \text { C } & 0.003330 & 0.049081 & 1.711334 \\ \text { C } & 1.192384 & 0.033683 & 2.474323 \\ \text { C } & 2.417896 & 0.121946 & 1.775102 \\ \text { C } & 2.448443 & 0.200451 & 0.401970 \\ \text { C } & 1.264105 & 0.201762 & -0.329731 \\ \text { C } & 1.186235 & -0.101633 & 3.878933 \\ \text { C } & -0.018392 & -0.298427 & 4.679857 \\ \text { C } & -0.696525 & -1.632891 & 4.701781 \\ \text { N } & 0.185213 & -2.724679 & 5.130754 \\ \text { S } & 0.474568 & -3.974251 & 4.126677 \\ \text { O } & -0.749731 & -4.288505 & 3.450032 \\ \text { C } & 1.163572 & -2.444609 & 6.200156 \\ \text { C } & 0.606976 & -1.414110 & 7.123926 \\ \text { C } & 0.677426 & -0.124087 & 6.804375 \\ \text { C } & 0.945908 & 1.148677 & 6.959201 \\ \text { O } & 1.135187 & -4.986569 & 4.897779 \\ \text { C } & 1.616139 & -3.403692 & 2.910228 \\ \text { H } & -1.086863 & -1.892499 & 3.711230 \\ \text { H } & -1.561581 & -1.586690 & 5.370254 \\ \text { H } & 1.893870 & -4.260619 & 2.297154 \\ \text { H } & 1.135294 & -2.641282 & 2.297044 \\ \text { H } & 2.497560 & -2.996190 & 3.406712 \\ \text { H } & 2.151681 & -0.052984 & 4.382194 \\ \text { H } & -0.708436 & 0.540443 & 4.774680\end{array}$




$\begin{array}{rrrr}H & 3.341688 & 0.113486 & 2.345360 \\ H & 3.400901 & 0.260548 & -0.112260 \\ H & 1.291613 & 0.260630 & -1.411048 \\ H & -0.879425 & 0.139892 & -0.228565 \\ H & -0.953805 & 0.001522 & 2.220008 \\ H & 1.364720 & -3.374684 & 6.728856 \\ H & 2.109640 & -2.088839 & 5.769198 \\ H & -0.033723 & -1.738543 & 7.939399 \\ H & 1.665943 & 1.466589 & 7.707160 \\ H & 0.495519 & 1.921259 & 6.347109\end{array}$

\section{${ }^{3} \mid I^{\prime}$}

35

scf done: -1146.761413

$\begin{array}{llll}\text { C } & 0.164627 & 1.662493 & 0.688619\end{array}$

C $0.052808 \quad 0.957120 \quad 1.866063$

$\begin{array}{llll}\text { C } & 1.042041 & 0.020660 & 2.251056\end{array}$

$\begin{array}{llll}\text { C } & 2.141792 & -0.151695 & 1.373371\end{array}$

$\begin{array}{llll}\text { C } & 2.241367 & 0.556852 & 0.199932\end{array}$

$\begin{array}{llll}\text { C } & 1.253901 & 1.472486 & -0.155124\end{array}$

$\begin{array}{llll}\text { C } & 0.988497 & -0.731495 & 3.435609\end{array}$

$\begin{array}{llll}\text { C } & -0.060557 & -0.682791 & 4.488268\end{array}$

$\begin{array}{llll}\text { C } & -0.668351 & -2.052171 & 4.767496\end{array}$

$\begin{array}{llll}\mathrm{N} & 0.320496 & -2.950944 & 5.341301\end{array}$

S $\quad 0.587781 \quad-4.433337 \quad 4.733507$

$\begin{array}{llll}\text { O } & -0.595611 & -4.856425 & 4.044387\end{array}$

C $1.062181-2.511616 \quad 6.518430$

C $1.082213 \quad-1.037623 \quad 6.663997$

C $\quad 0.563273 \quad-0.136154 \quad 5.756430$

C $\quad 0.628104 \quad 1.227825 \quad 5.942944$

$\begin{array}{llll}\text { O } & 1.122026 & -5.239952 & 5.791557\end{array}$

C $1.854829-4.234220 \quad 3.526712$

H $\quad-1.052204 \quad-2.496288 \quad 3.849173$

H $\quad-1.511087 \quad-1.939423 \quad 5.460389$

H $\quad 2.084273 \quad-5.218118 \quad 3.118901$

H $\quad \begin{array}{llll}1.489823 & -3.574023 & 2.739417\end{array}$

H $\quad 2.737484-3.811899 \quad 4.007298$

H $\quad \begin{array}{llll}1.843753 & -1.368835 & 3.651249\end{array}$

H $\quad-0.878765 \quad-0.022346 \quad 4.189163$

H $2.913093 \quad-0.865281 \quad 1.647296$

H $\quad 3.094269 \quad 0.401894 \quad-0.450939$

H $\quad 1.333688 \quad 2.032702 \quad-1.078936$

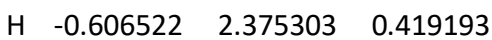

$\begin{array}{llll}H & -0.806086 & 1.127400 & 2.504412\end{array}$

H $\quad 0.644363 \quad-2.966771 \quad 7.427911$

H $\quad 2.092412 \quad-2.887467 \quad 6.455178$

H $\quad \begin{array}{llll}1.547822 & -0.663793 & 7.570786\end{array}$

H $\quad 1.098103 \quad 1.650514 \quad 6.823506$

H $\quad 0.216943 \quad 1.912242 \quad 5.209390$

\section{2}

35

scf done: -1146.816233

$\begin{array}{llll}\text { C } & 1.634281 & -4.107607 & 23.097796\end{array}$

$\begin{array}{llll}\text { C } & 1.468107 & -3.164085 & 24.278012\end{array}$

$\begin{array}{llll}\text { C } & 2.816536 & -2.391700 & 24.383750\end{array}$

$\begin{array}{llll}\text { C } & 3.657315 & -2.875177 & 23.224925\end{array}$

N $3.093201 \quad-4.206588 \quad 22.964190$

C $1.744133 \quad-3.75078925 .639009$

$\begin{array}{llll}\text { C } & 1.038997 & -4.500924 & 26.462531\end{array}$

$\begin{array}{llll}\text { C } & 3.104406 & -3.089630 & 25.746157\end{array}$

$\begin{array}{llll}\text { C } & 3.368388 & -2.218608 & 26.932889\end{array}$

C $2.440338-1.258873 \quad 27.331832$ 


$\begin{array}{llll}\text { C } & 2.689719 & -0.439051 & 28.416912 \\ \text { C } & 3.874025 & -0.565493 & 29.128654 \\ \text { C } & 4.802774 & -1.517084 & 28.744689 \\ \text { C } & 4.549378 & -2.335980 & 27.654944 \\ \text { S } & 3.731471 & -5.136070 & 21.786649 \\ \text { O } & 5.153550 & -5.118581 & 21.959031 \\ \text { C } & 3.360456 & -4.326587 & 20.267091 \\ \text { O } & 3.028832 & -6.384316 & 21.805957 \\ \text { H } & 2.755352 & -1.306064 & 24.441860 \\ \text { H } & 3.528602 & -2.215281 & 22.355129 \\ \text { H } & 4.720667 & -2.952532 & 23.453026 \\ \text { H } & 0.552677 & -2.576124 & 24.200705 \\ \text { H } & 3.916030 & -3.819607 & 25.642956 \\ \text { H } & 1.198682 & -5.092641 & 23.264250 \\ \text { H } & 1.184160 & -3.667573 & 22.196543 \\ \text { H } & 0.049767 & -4.858902 & 26.197171 \\ \text { H } & 1.423122 & -4.780372 & 27.438427 \\ \text { H } & 5.280139 & -3.081149 & 27.356699 \\ \text { H } & 1.507864 & -1.157202 & 26.783590 \\ \text { H } & 5.730198 & -1.625657 & 29.295377 \\ \text { H } & 4.069056 & 0.075132 & 29.980668 \\ \text { H } & 1.956079 & 0.302360 & 28.712158 \\ \text { H } & 3.773103 & -4.936148 & 19.464486 \\ \text { H } & 2.279953 & -4.247698 & 20.154879 \\ \text { H } & 3.824736 & -3.341356 & 20.260769\end{array}$

\section{$2^{\prime}$}

35

scf done: -1146.810608

$\begin{array}{llll}\text { C } & 0.038627 & 2.236763 & 1.028448\end{array}$

$\begin{array}{llll}\text { C } & 0.256857 & 1.577390 & 2.225225\end{array}$

C $1.008204 \quad 0.405942 \quad 2.268121$

$\begin{array}{llll}\text { C } & 1.530131 & -0.086326 & 1.077004\end{array}$

$\begin{array}{llll}\text { C } & 1.312503 & 0.569407 & -0.123961\end{array}$

C $\quad 0.566004 \quad 1.734910 \quad-0.151702$

C $1.226132 \quad-0.336088 \quad 3.543909$

$\begin{array}{llll}\text { C } & -0.007944 & -0.909997 & 4.303442\end{array}$

$\begin{array}{llll}\text { C } & -0.509292 & -2.338264 & 4.141308\end{array}$

$\begin{array}{llll}\mathrm{N} & -0.033009 & -3.224681 & 5.207292\end{array}$

S $0.981693 \quad-4.456377 \quad 4.887998$

$\begin{array}{llll}\text { O } & 0.716109 & -4.893702 & 3.548449\end{array}$

C $\quad-0.032620 \quad-2.645748 \quad 6.560986$

C $0.821939-1.409715 \quad 6.603799$

C $0.754325 \quad-0.609803 \quad 5.555364$

C $1.612360 \quad 0.402593 \quad 4.859843$

$\begin{array}{llll}\text { O } & 0.876014 & -5.395995 & 5.966514\end{array}$

C $2.623741 \quad-3.816062 \quad 4.929722$

H $\quad-0.212092 \quad-2.756145 \quad 3.178188$

$\begin{array}{llll}\mathrm{H} & -1.602867 & -2.353935 & 4.173597\end{array}$

H $\quad 3.304661 \quad-4.642336 \quad 4.727880$

H $\quad 2.726990 \quad-3.046848 \quad 4.165677$

H $\quad 2.818717-3.400825 \quad 5.917762$

H $\quad \begin{array}{llll}1.941141 & -1.148112 & 3.375537\end{array}$

H $\quad-0.836360-0.204303 \quad 4.175229$

H $\quad 2.118670 \quad-0.998574 \quad 1.095088$

H $\quad 1.7291550 .168070 \quad-1.040746$

H $\quad 0.3949892 .252076 \quad-1.088650$

H $\quad-0.547909 \quad 3.148355 \quad 1.015999$

H $\quad-0.165611 \quad 1.983088 \quad 3.139526$

H $\quad-1.077117 \quad-2.437935 \quad 6.821144$

$\begin{array}{llll}\text { H } & 0.316888 & -3.403907 & 7.260443\end{array}$

H $\quad \begin{array}{llll}1.523581 & -1.264785 & 7.418133\end{array}$

H $\quad 2.667674 \quad 0.459673 \quad 5.126063$ 


\section{3}

32

scf done: -1107.482285

C $\quad 1.121650 \quad-0.252960 \quad 2.153346$

$\begin{array}{llll}\text { C } & 2.324264 & -0.337037 & 1.328678\end{array}$

$\begin{array}{llll}\text { C } & 2.488491 & 0.150199 & 0.101951\end{array}$

$\begin{array}{llll}\text { C } & 3.753709 & 0.037124 & -0.677784\end{array}$

$\begin{array}{llll}\text { N } & 4.508422 & 1.298437 & -0.777752\end{array}$

S $3.936981 \quad 2.520775 \quad-1.727053$

$\begin{array}{llll}\text { C } & 2.900808 & 3.495707 & -0.688324\end{array}$

C $5.395464 \quad 1.653561 \quad 0.258610$

C $5.672042 \quad 0.927086 \quad 1.305585$

$\begin{array}{llll}\text { O } & 3.132687 & 1.905201 & -2.737348\end{array}$

$\begin{array}{llll}\text { O } & 5.053709 & 3.336239 & -2.096301\end{array}$

H $3.557168 \quad-0.302551 \quad-1.694645$

H $4.434317-0.680111-0.214029$

H $\quad 2.555200 \quad 4.342262 \quad-1.280573$

$\begin{array}{llll}H & 2.056510 & 2.896789 & -0.351598\end{array}$

H $3.483453 \quad 3.848046 \quad 0.162103$

$\begin{array}{llll}H & 5.896013 & 2.606018 & 0.111081\end{array}$

H $\quad 3.157844 \quad-0.873473 \quad 1.781373$

$\begin{array}{llll}\text { C } & 6.005867 & 0.229016 & 2.346451\end{array}$

$\begin{array}{llll}\text { H } & 6.819552 & -0.491022 & 2.317027\end{array}$

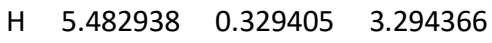

H $\quad 1.666650 \quad 0.656340 \quad-0.400420$

$\begin{array}{llll}\text { C } & 1.051440 & -1.007967 & 3.323021\end{array}$

$\begin{array}{llll}\text { C } & -0.070531 & -0.969554 & 4.132581\end{array}$

$\begin{array}{llll}\text { C } & -1.144648 & -0.166487 & 3.789801\end{array}$

$\begin{array}{llll}\text { C } & -1.085490 & 0.600177 & 2.634213\end{array}$

$\begin{array}{llll}\text { C } & 0.033780 & 0.559027 & 1.826682\end{array}$

H $\quad 1.893719-1.636745 \quad 3.593637$

H $\quad-0.104455-1.568385 \quad 5.035265$

H $-2.023920 \quad-0.131296 \quad 4.422122$

$\begin{array}{llll}H & -1.918663 & 1.239019 & 2.364977\end{array}$

H $\quad 0.066188 \quad 1.1753380 .934604$

${ }^{3} 3$

32

scf done: -1107.401767

$\begin{array}{llll}\text { C } & 1.426010 & -0.023837 & 2.038877\end{array}$

C $2.755814 \quad-0.335832 \quad 1.694543$

$\begin{array}{llll}\text { C } & 3.172139 & -0.810063 & 0.390230\end{array}$

$\begin{array}{llll}\text { C } & 3.447824 & 0.094688 & -0.762208\end{array}$

$\begin{array}{llll}\text { N } & 4.054792 & 1.369921 & -0.385123\end{array}$

$\begin{array}{lllll}\text { S } & 3.254003 & 2.802344 & -0.548788\end{array}$

C $2.452572 \quad 3.092791 \quad 0.989607$

C $5.274881 \quad 1.360953 \quad 0.314618$

$\begin{array}{llll}\text { C } & 5.927483 & 0.286530 & 0.663841\end{array}$

$\begin{array}{llll}\text { O } & 2.253060 & 2.609845 & -1.552969\end{array}$

$\begin{array}{lllll}O & 4.245952 & 3.821499 & -0.714562\end{array}$

H $2.535873 \quad 0.333128-1.320704$

H $4.122191 \quad-0.402229 \quad-1.468882$

H $\quad 2.077420 \quad 4.115481 \quad 0.969019$

H $1.631438 \quad 2.386201 \quad 1.104195$

H $3.179686 \quad 2.971180 \quad 1.793363$

H $\quad 5.679990 \quad 2.343182 \quad 0.534860$

H $\quad 3.515652 \quad-0.199516 \quad 2.467279$

$\begin{array}{llll}\text { C } & 6.612791 & -0.749462 & 1.038633\end{array}$

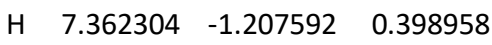

H $6.459293 \quad-1.210448 \quad 2.011850$

H $\quad 3.243932 \quad-1.877806 \quad 0.195674$ 


$\begin{array}{rrrr}\text { C } & 1.117297 & 0.486619 & 3.320739 \\ \text { C } & -0.166038 & 0.861799 & 3.643714 \\ \text { C } & -1.191837 & 0.739537 & 2.709880 \\ \text { C } & -0.914674 & 0.226086 & 1.446431 \\ \text { C } & 0.366364 & -0.152793 & 1.111733 \\ \text { H } & 1.919186 & 0.591155 & 4.044955 \\ \text { H } & -0.379170 & 1.257577 & 4.630165 \\ \text { H } & -2.200582 & 1.040374 & 2.966095 \\ \text { H } & -1.712301 & 0.123350 & 0.719558 \\ \text { H } & 0.577816 & -0.556481 & 0.126067\end{array}$

\section{${ }^{2} \mathrm{Ms}$}

7

scf done: -588.481609

C $2.757171 \quad 3.451048 \quad-0.312669$

$\begin{array}{llll}\text { S } & 3.843311 & 2.571298 & -1.440555\end{array}$

$\begin{array}{lllll}\text { O } & 4.930289 & 3.466043 & -1.793198\end{array}$

$\begin{array}{llll}\text { O } & 3.019126 & 1.995476 & -2.487148\end{array}$

H $\quad 2.332735 \quad 4.277833 \quad-0.882707$

H $\quad 1.986252 \quad 2.759250 \quad 0.016321$

H $\quad 3.358763 \quad 3.810305 \quad 0.518005$

\section{TS (3-III)}

32

scf done: -1107.391966

$\begin{array}{llll}\text { C } & -0.087498 & 0.252109 & 0.063188\end{array}$

$\begin{array}{llll}\text { C } & 0.034324 & -0.074877 & 1.432691\end{array}$

C $1.337825 \quad-0.1297891 .977065$

$\begin{array}{llll}\text { C } & 2.444544 & 0.121024 & 1.199321\end{array}$

$\begin{array}{llll}\text { C } & 2.299855 & 0.439480 & -0.148145\end{array}$

$\begin{array}{llll}\text { C } & 1.027386 & 0.505076 & -0.705252\end{array}$

$\begin{array}{llll}\text { C } & -1.085307 & -0.352642 & 2.245532\end{array}$

$\begin{array}{llll}\text { C } & -2.458826 & -0.422676 & 1.759187\end{array}$

$\begin{array}{llll}\text { C } & -2.920885 & -1.611110 & 0.981093\end{array}$

$\begin{array}{llll}\text { N } & -2.999776 & -2.780598 & 1.870503\end{array}$

$\begin{array}{llll}\text { C } & -3.368443 & -2.450385 & 3.183534\end{array}$

$\begin{array}{llll}\text { C } & -3.544147 & -1.203708 & 3.561365\end{array}$

$\begin{array}{llll}\text { C } & -3.968135 & -0.206632 & 4.327283\end{array}$

$\begin{array}{llll}\text { S } & -2.084700 & -4.122612 & 1.594886\end{array}$

$\begin{array}{llll}\text { O } & -2.536341 & -5.122602 & 2.513625\end{array}$

C $-0.436249 \quad-3.682258 \quad 2.028493$

$\begin{array}{llll}\text { O } & -2.126758 & -4.371196 & 0.186910\end{array}$

H $\quad-2.251667 \quad-1.858938 \quad 0.150355$

H $\quad-3.914951 \quad-1.4297890 .564338$

H $\quad 0.172311-4.583175 \quad 1.957539$

H $\quad-0.075825 \quad-2.926045 \quad 1.331820$

H $\quad-0.431265 \quad-3.297500 \quad 3.048950$

$\begin{array}{llll}H & -3.493207 & -3.283618 & 3.864886\end{array}$

$\begin{array}{llll}H & -0.898924 & -0.521651 & 3.306156\end{array}$

$\begin{array}{llll}\mathrm{H} & -5.021422 & 0.046198 & 4.407676\end{array}$

$\begin{array}{llll}H & -3.265478 & 0.469060 & 4.808483\end{array}$

$\begin{array}{llll}H & -2.986616 & 0.507655 & 1.560626\end{array}$

H $1.450812 \quad-0.381132 \quad 3.027319$

$\begin{array}{llll}\text { H } & 3.434095 & 0.070150 & 1.639027\end{array}$

H $\quad 3.172771 \quad 0.638232 \quad-0.758175$

H $\quad 0.910268 \quad 0.760605 \quad-1.752256$

H $\quad-1.0758780 .321458 \quad-0.379561$

\section{${ }^{3}$ III}

32

scf done: -1107.471149

C $\quad-3.462008-2.370116 \quad-1.108423$ 


$\begin{array}{llll}\text { C } & -3.878110 & -3.223579 & -2.307371 \\ \text { C } & -4.293917 & -2.172694 & -3.338947 \\ \text { N } & -3.520731 & -0.983867 & -2.940348 \\ \text { C } & -3.234311 & -1.084728 & -1.576530 \\ \text { C } & -4.940979 & -4.212037 & -2.017749 \\ \text { C } & -4.753283 & -5.575154 & -1.738643 \\ \text { C } & -3.482507 & -6.199615 & -1.715113 \\ \text { C } & -3.357289 & -7.541026 & -1.431179 \\ \text { C } & -4.478472 & -8.317963 & -1.158525 \\ \text { C } & -5.739691 & -7.728094 & -1.174194 \\ \text { C } & -5.877409 & -6.389765 & -1.456127 \\ \text { S } & -3.831720 & 0.480787 & -3.632970 \\ \text { O } & -4.004141 & 0.236480 & -5.030834 \\ \text { C } & -3.306493 & -2.837455 & 0.162304 \\ \text { C } & -5.369817 & 0.996222 & -2.951489 \\ \text { O } & -2.815229 & 1.375447 & -3.174166 \\ \text { H } & -2.974028 & -3.725621 & -2.670856 \\ \text { H } & -2.827226 & -0.236377 & -1.045055 \\ \text { H } & -5.369474 & -1.967390 & -3.252968 \\ \text { H } & -4.070466 & -2.452511 & -4.365922 \\ \text { H } & -5.956911 & -3.825228 & -1.961497 \\ \text { H } & -6.861632 & -5.932411 & -1.466540 \\ \text { H } & -6.618509 & -8.325954 & -0.961299 \\ \text { H } & -4.370635 & -9.372324 & -0.933843 \\ \text { H } & -2.372444 & -7.994121 & -1.417970 \\ \text { H } & -2.592047 & -5.615148 & -1.917854 \\ \text { H } & -5.595166 & 1.978388 & -3.365032 \\ \text { H } & -6.145956 & 0.286390 & -3.233443 \\ \text { H } & -5.270581 & 1.055785 & -1.868128 \\ \text { H } & -3.523966 & -3.869851 & 0.407475 \\ \text { H } & -2.972045 & -2.186029 & 0.960382\end{array}$

\section{TS (III-IV)}

32

scf done: -1107.437330

$\begin{array}{llll}\text { C } & -0.718373 & -0.106016 & 0.380737\end{array}$

$\begin{array}{llll}\text { C } & -0.278107 & -0.045284 & 1.725794\end{array}$

C $1.118535-0.106509 \quad 1.950476$

$\begin{array}{llll}\text { C } & 2.001621 & -0.216036 & 0.899576\end{array}$

$\begin{array}{llll}\text { C } & 1.542265 & -0.271166 & -0.411912\end{array}$

$\begin{array}{llll}\text { C } & 0.173011 & -0.215601 & -0.659933\end{array}$

$\begin{array}{llll}\text { C } & -1.220314 & 0.075248 & 2.759487\end{array}$

C $\quad-0.925722 \quad 0.093823 \quad 4.216200$

C $-1.134281 \quad-1.295880 \quad 4.801174$

C $\quad-2.338361 \quad-1.219553 \quad 5.580155$

$\begin{array}{llll}\mathrm{N} & -2.827691 & -0.019390 & 5.704433\end{array}$

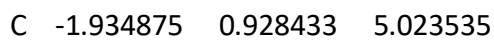

$\begin{array}{llll}\text { C } & -0.372772 & -2.378525 & 4.627517\end{array}$

$\begin{array}{llll}\text { S } & -4.917100 & 0.225169 & 5.089788\end{array}$

$\begin{array}{llll}\text { O } & -5.525365 & -0.914679 & 5.737206\end{array}$

$\begin{array}{llll}\text { O } & -5.270986 & 1.555808 & 5.530546\end{array}$

C $\quad-5.269549 \quad 0.100593 \quad 3.333034$

$\begin{array}{llll}\mathrm{H} & 0.093244 & 0.437757 & 4.404242\end{array}$

$\begin{array}{llll}\text { H } & -2.832229 & -2.072657 & 6.036779\end{array}$

$\begin{array}{llll}\mathrm{H} & -2.516136 & 1.615572 & 4.402881\end{array}$

H $\quad-1.441733 \quad 1.529976 \quad 5.790465$

$\begin{array}{llll}H & -2.272702 & 0.077694 & 2.478527\end{array}$

H $\quad-1.784876-0.0630050 .183822$

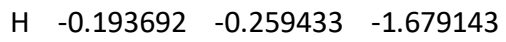

H $\quad 2.243725 \quad-0.358246-1.232916$

H $3.066273 \quad-0.259925 \quad 1.099370$

H $\quad \begin{array}{llll}1.503797 & -0.065400 & 2.963107\end{array}$

$\begin{array}{llll}H & -6.355297 & 0.096524 & 3.224283\end{array}$ 


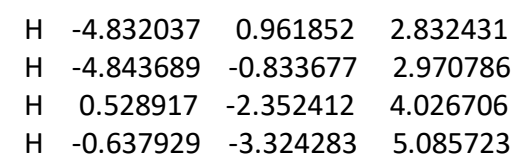

${ }^{3} \mathrm{IV}$

scf done: -1107.447628

C $\quad 0.365505 \quad-0.290033 \quad-0.152482$

$\begin{array}{llll}\text { C } & 0.278789 & -0.161691 & 1.255667\end{array}$

$\begin{array}{llll}\text { C } & 1.497417 & -0.105028 & 1.974334\end{array}$

$\begin{array}{llll}\text { C } & 2.708766 & -0.164373 & 1.321822\end{array}$

C $2.764852 \quad-0.282903 \quad-0.063144$

$\begin{array}{llll}\text { C } & 1.580609 & -0.346807 & -0.792843\end{array}$

$\begin{array}{llll}\text { C } & -0.982588 & -0.101448 & 1.869524\end{array}$

$\begin{array}{llll}\text { C } & -1.238816 & 0.037879 & 3.321486\end{array}$

C $-1.451518-1.278092 \quad 4.048842$

$\begin{array}{llll}\text { C } & -2.607142 & -1.052198 & 4.908347\end{array}$

$\begin{array}{llll}\text { N } & -3.200744 & 0.058690 & 4.740571\end{array}$

$\begin{array}{llll}\text { C } & -2.530751 & 0.800363 & 3.675588\end{array}$

$\begin{array}{llll}\text { C } & -0.774676 & -2.412121 & 3.929213\end{array}$

$\begin{array}{llll}\text { S } & -5.386722 & 0.185493 & 6.728502\end{array}$

$\begin{array}{llll}\text { O } & -5.358071 & -1.261775 & 6.859030\end{array}$

$\begin{array}{llll}0 & -5.899493 & 0.978338 & 7.836233\end{array}$

C $\quad-6.381078 \quad 0.579962 \quad 5.288665$

$\begin{array}{llll}\text { H } & -0.394230 & 0.542548 & 3.805550\end{array}$

H $\quad-2.964853 \quad-1.783819 \quad 5.632100$

$\begin{array}{llll}H & -3.212076 & 0.858286 & 2.819244\end{array}$

$\begin{array}{llll}H & -2.344132 & 1.826690 & 3.996501\end{array}$

H $\quad-1.855481 \quad-0.207326 \quad 1.228568$

$\begin{array}{llll}H & -0.555880 & -0.340231 & -0.724024\end{array}$

H $1.615995 \quad-0.441895-1.872123$

H $3.722146-0.328462 \quad-0.568501$

$\begin{array}{llll}H & 3.627407 & -0.120893 & 1.895996\end{array}$

H $\quad 1.478059 \quad-0.024058 \quad 3.055763$

$\begin{array}{llll}H & -7.402838 & 0.283017 & 5.525995\end{array}$

$\begin{array}{llll}H & -6.312100 & 1.651559 & 5.118340\end{array}$

$\begin{array}{llll}H & -5.976018 & 0.016670 & 4.452726\end{array}$

H $0.050336-2.516396 \quad 3.232950$

H $\quad-1.033094 \quad-3.279170 \quad 4.526433$

\section{TS (3-V)}

39

scf done: -1695.970008

$\begin{array}{llll}\text { C } & 1.612130 & -1.072801 & 0.310633\end{array}$

C $1.080199-0.893900 \quad 1.572518$

$\begin{array}{llll}\text { C } & 1.631039 & 0.037845 & 2.453501\end{array}$

C $2.724408 \quad 0.787014 \quad 2.021203$

$\begin{array}{llll}\text { C } & 3.259485 & 0.608478 & 0.757794\end{array}$

$\begin{array}{llll}\text { C } & 2.705465 & -0.323919 & -0.102767\end{array}$

$\begin{array}{llll}\text { C } & 1.128899 & 0.247399 & 3.806916\end{array}$

$\begin{array}{llll}\text { C } & 0.155826 & -0.421044 & 4.418175\end{array}$

$\begin{array}{llll}\text { C } & -0.304353 & -0.113779 & 5.800329\end{array}$

$\begin{array}{llll}\text { N } & -0.156978 & -1.241531 & 6.735727\end{array}$

S $-1.404020 \quad-2.331892 \quad 6.966062$

$\begin{array}{llll}0 & -2.605440 & -1.650316 & 6.603675\end{array}$

C $1.078248-1.548775 \quad 7.267505$

$\begin{array}{llll}\text { C } & 2.237008 & -1.010356 & 6.855636\end{array}$

S $\quad 2.966107 \quad-2.587380 \quad 5.065419$

C $3.774205 \quad-3.757576 \quad 6.153756$

$\begin{array}{llll}\text { C } & 3.242986 & -0.183710 & 6.889498\end{array}$

$\begin{array}{llll}\text { O } & -1.246475 & -2.867916 & 8.281608\end{array}$

C $\quad-1.086312-3.615580 \quad 5.811654$ 


$\begin{array}{rrrr}\mathrm{O} & 1.856493 & -3.302424 & 4.448758 \\ \mathrm{O} & 3.998183 & -2.033844 & 4.196965 \\ \mathrm{H} & -1.358569 & 0.165361 & 5.813783 \\ \mathrm{H} & 0.261832 & 0.719695 & 6.222786 \\ \mathrm{H} & -1.816604 & -4.402484 & 5.998287 \\ \mathrm{H} & -1.193754 & -3.224263 & 4.801717 \\ \mathrm{H} & -0.070705 & -3.980325 & 5.965795 \\ \mathrm{H} & 1.081985 & -2.350776 & 8.000128 \\ \mathrm{H} & 1.628935 & 1.039354 & 4.364293 \\ \mathrm{H} & -0.364104 & -1.228230 & 3.906928 \\ \mathrm{H} & 3.163073 & 1.513289 & 2.698387 \\ \mathrm{H} & 4.112742 & 1.199073 & 0.445025 \\ \mathrm{H} & 3.120709 & -0.467428 & -1.093383 \\ \mathrm{H} & 1.170893 & -1.801407 & -0.359613 \\ \mathrm{H} & 0.223395 & -1.485563 & 1.875879 \\ \mathrm{H} & 4.085470 & -0.263554 & 6.208666 \\ \mathrm{H} & 3.266496 & 0.631666 & 7.607390 \\ \mathrm{H} & 4.162659 & -4.564201 & 5.531501 \\ \mathrm{H} & 4.578762 & -3.241588 & 6.674535 \\ \mathrm{H} & 3.028077 & -4.131906 & 6.853421\end{array}$

\section{${ }^{2} \mathrm{~V}$}

39

scf done: -1696.007693

$\begin{array}{rrrr}\text { C } & -0.265965 & 0.700926 & 1.627030 \\ \text { C } & 0.800168 & -0.042937 & 2.137473 \\ \text { C } & 0.685987 & -0.559570 & 3.427359 \\ \text { C } & -0.455481 & -0.352878 & 4.181829\end{array}$

$\begin{array}{llll}\text { C } & -1.506172 & 0.381798 & 3.659444\end{array}$

$\begin{array}{llll}\text { C } & -1.404984 & 0.908889 & 2.379285\end{array}$

$\begin{array}{llll}\text { C } & 2.025828 & -0.292767 & 1.386074\end{array}$

C $2.260695-0.006183 \quad 0.107310$

C $3.580058 \quad-0.250441 \quad-0.539158$

$\begin{array}{llll}\text { N } & 4.471880 & 0.941687 & -0.482960\end{array}$

C $5.224808 \quad 1.222941 \quad 0.643655$

$\begin{array}{llll}\text { C } & 5.978256 & 0.298437 & 1.351458\end{array}$

S $\quad 4.109771 \quad 2.241769 \quad-1.468586$

O $5.316940 \quad 2.992397 \quad-1.614361$

$\begin{array}{llll}\text { C } & 2.969737 & 3.232099 & -0.562987\end{array}$

$\begin{array}{lllll}\mathrm{O} & 3.447113 & 1.700914 & -2.612924\end{array}$

H $3.479962-0.530981 \quad-1.585659$

H $\quad 4.104381 \quad-1.054860-0.027715$

H $\quad 2.773481 \quad 4.113240 \quad-1.173197$

H $\quad 2.050573 \quad 2.674741 \quad-0.393350$

H $3.419598 \quad 3.531459 \quad 0.382217$

$\begin{array}{llll}\text { H } & 5.249155 & 2.256202 & 0.971987\end{array}$

H $2.820121 \quad-0.778863 \quad 1.953512$

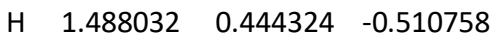

H $\quad \begin{array}{llll}1.510667 & -1.132932 & 3.838535\end{array}$

H $\quad-0.523113 \quad-0.765009 \quad 5.181865$

H $\quad-2.400658 \quad 0.549646 \quad 4.247530$

$\begin{array}{llll}H & -2.221261 & 1.491035 & 1.967694\end{array}$

$\begin{array}{llll}\mathrm{H} & -0.203041 & 1.128250 & 0.632009\end{array}$

$\begin{array}{llll}\text { C } & 6.333009 & -0.985093 & 1.022183\end{array}$

S $\quad \begin{array}{llll}\text { C } & 652123 & 0.919526 & 2.883853\end{array}$

H $6.905732 \quad-1.594127 \quad 1.709033$

H $6.101936 \quad-1.4087390 .054036$

$\begin{array}{llll}\text { O } & 5.993979 & 2.154269 & 3.217349\end{array}$

$\begin{array}{llll}\text { O } & 6.645190 & -0.149987 & 3.844338\end{array}$

$\begin{array}{llll}\text { C } & 8.325383 & 1.282697 & 2.470380\end{array}$

H $\quad 8.794903 \quad 1.667639 \quad 3.375575$

$\begin{array}{llll}H & 8.818397 & 0.368912 & 2.143207\end{array}$

$\begin{array}{llll}\text { H } & 8.339393 & 2.035601 & 1.683738\end{array}$ 


\begin{tabular}{cccc}
\multicolumn{5}{c}{ TS $(\mathrm{V}-\mathrm{VI})$} \\
39 \\
scf done: -1695.973466 \\
C & -0.043280 & 0.488725 & 0.334585 \\
C & 0.237496 & -0.844019 & 0.580499 \\
C & 1.397309 & -1.194330 & 1.249599 \\
C & 2.299656 & -0.224656 & 1.685437 \\
C & 2.000702 & 1.115465 & 1.431957 \\
C & 0.844303 & 1.466096 & 0.764123 \\
C & 3.503905 & -0.657016 & 2.388959 \\
C & 4.465675 & 0.114208 & 2.887364 \\
C & 5.670037 & -0.355556 & 3.632387 \\
N & 5.634071 & -1.722247 & 4.096634 \\
C & 5.999094 & -2.761646 & 3.421737 \\
C & 6.525201 & -2.845259 & 2.076859 \\
C & 6.439729 & -1.983324 & 1.058560 \\
S & 7.434133 & -4.355461 & 1.773083 \\
O & 7.828909 & -4.383916 & 0.392735 \\
C & 8.879437 & -4.180429 & 2.761693 \\
O & 6.652389 & -5.443954 & 2.296100 \\
S & 4.054602 & -1.999549 & 5.528235 \\
C & 2.277015 & -1.888317 & 5.235619 \\
O & 4.398934 & -0.953622 & 6.463725 \\
O & 4.332633 & -3.375629 & 5.872608 \\
H & 5.796893 & 0.276422 & 4.517480 \\
H & 6.573021 & -0.212064 & 3.022225 \\
H & 1.798381 & -2.161630 & 6.177566 \\
H & 2.039377 & -0.865127 & 4.953290 \\
H & 2.012540 & -2.591807 & 4.448653 \\
H & 5.940385 & -3.702681 & 3.970651 \\
H & 3.586635 & -1.737320 & 2.508887 \\
H & 4.418445 & 1.193580 & 2.772936 \\
H & 2.679499 & 1.894662 & 1.760446 \\
H & 0.630069 & 2.511812 & 0.575948 \\
H & -0.949534 & 0.768635 & -0.189373 \\
H & -0.448108 & -1.615497 & 0.249995 \\
H & 1.617558 & -2.240495 & 1.439228 \\
H & 6.937961 & -2.198276 & 0.121898 \\
H & 5.838139 & -1.085905 & 1.110843 \\
H & 9.464693 & -5.088795 & 2.618433 \\
H & 9.436931 & -3.309106 & 2.422044 \\
H & 8.593335 & -4.078516 & 3.807577 \\
& & & \\
\end{tabular}

\section{VI \\ 32}

scf done: -1107.494400

$\begin{array}{rrrr}\text { C } & -1.567891 & 0.261787 & 1.905483 \\ \text { C } & -0.265743 & 0.323464 & 1.450031 \\ \text { C } & 0.735549 & -0.452210 & 2.038283 \\ \text { C } & 0.379372 & -1.293526 & 3.091554 \\ \text { C } & -0.925510 & -1.357043 & 3.548967 \\ \text { C } & -1.905305 & -0.578100 & 2.957933 \\ \text { C } & 2.129248 & -0.420985 & 1.601135 \\ \text { C } & 2.665193 & 0.371648 & 0.677780 \\ \text { C } & 4.098579 & 0.317526 & 0.271224 \\ \text { N } & 4.670075 & 1.651226 & 0.284580 \\ \text { C } & 5.523405 & 1.997056 & 1.147926 \\ \text { C } & 6.057218 & 1.201536 & 2.276342 \\ \text { S } & 7.825921 & 1.082984 & 2.348405 \\ \text { C } & 8.199701 & 0.058907 & 0.967072 \\ \text { C } & 5.343551 & 0.655980 & 3.247650 \\ \text { O } & 8.356471 & 2.396939 & 2.099263\end{array}$




$\begin{array}{rrrr}\mathrm{O} & 8.205648 & 0.394155 & 3.549678 \\ \mathrm{H} & 4.161264 & -0.030121 & -0.767373 \\ \mathrm{H} & 4.642310 & -0.405224 & 0.892883 \\ \mathrm{H} & 5.942672 & 3.004130 & 1.067976 \\ \mathrm{H} & 2.781562 & -1.136443 & 2.102180 \\ \mathrm{H} & 2.062893 & 1.115203 & 0.161303 \\ \mathrm{H} & 1.145823 & -1.905637 & 3.556496 \\ \mathrm{H} & -1.177148 & -2.018058 & 4.370248 \\ \mathrm{H} & -2.928616 & -0.625249 & 3.311179 \\ \mathrm{H} & -2.330088 & 0.871376 & 1.433813 \\ \mathrm{H} & -0.024782 & 0.979526 & 0.621028 \\ \mathrm{H} & 5.820344 & 0.126724 & 4.063915 \\ \mathrm{H} & 4.262636 & 0.747051 & 3.259565 \\ \mathrm{H} & 9.284992 & -0.033932 & 0.927769 \\ \mathrm{H} & 7.737088 & -0.916236 & 1.113647 \\ \mathrm{H} & 7.830811 & 0.534616 & 0.058825\end{array}$

\section{${ }^{3} \mathrm{VI}$}

32

scf done: -1107.411141

C $\quad-0.335546 \quad 1.307530 \quad 2.055636$

$\begin{array}{llll}\text { C } & 0.658388 & 0.689631 & 1.331131\end{array}$

C $\quad 1.190727 \quad-0.553314 \quad 1.744561$

C $0.666838 \quad-1.130330 \quad 2.924460$

$\begin{array}{llll}\text { C } & -0.324284 & -0.501668 & 3.642291\end{array}$

$\begin{array}{llll}\text { C } & -0.835093 & 0.720814 & 3.214709\end{array}$

$\begin{array}{llll}\text { C } & 2.204769 & -1.208109 & 1.017636\end{array}$

$\begin{array}{llll}\text { C } & 2.835613 & -0.691476 & -0.179455\end{array}$

$\begin{array}{llll}C & 4.138157 & 0.033749 & -0.108588\end{array}$

$\begin{array}{llll}\mathrm{N} & 3.976470 & 1.294495 & 0.598105\end{array}$

C $4.481702 \quad 1.480940 \quad 1.740131$

C $5.295671 \quad 0.545343 \quad 2.554315$

S $\quad 6.974836 \quad 1.058254 \quad 2.815728$

$\begin{array}{llll}\text { C } & 7.709559 & 0.832270 & 1.232491\end{array}$

C $4.868265 \quad-0.547744 \quad 3.163688$

$\begin{array}{llll}\text { O } & 6.948319 & 2.466785 & 3.108524\end{array}$

$\begin{array}{llll}\mathrm{O} & 7.605288 & 0.164646 & 3.746341\end{array}$

H $4.480655 \quad 0.274339 \quad-1.117624$

$\begin{array}{llll}\text { H } & 4.896812 & -0.616218 & 0.357434\end{array}$

$\begin{array}{llll}H & 4.340230 & 2.464916 & 2.196384\end{array}$

H $\quad 2.520142 \quad-2.187896 \quad 1.384510$

H $2.425775 \quad-0.927276 \quad-1.158144$

H $\quad \begin{array}{llll}1.063412 & -2.085000 & 3.257112\end{array}$

H $\quad-0.710128 \quad-0.961571 \quad 4.545019$

$\begin{array}{llll}\mathrm{H} & -1.617633 & 1.212438 & 3.780287\end{array}$

H $\quad-0.733046 \quad 2.258525 \quad 1.719252$

H $\quad 1.049527 \quad 1.147160 \quad 0.427634$

H $\quad 5.535537 \quad-1.140279 \quad 3.778382$

H $3.834190 \quad-0.865183 \quad 3.078724$

$\begin{array}{llll}H & 8.735653 & 1.192459 & 1.309507\end{array}$

$\begin{array}{llll}\text { H } & 7.694187 & -0.227368 & 0.982109\end{array}$

H $\quad 7.162245 \quad 1.418214 \quad 0.494475$

\section{TS (VI-VII)}

32

scf done: -1107.398933
C $\quad-0.070044-0.179135$
0.127847
C $\quad 0.054283 \quad 0.109440 \quad 1.484454$
$\begin{array}{llll}\text { C } & 1.319997 & 0.270603 & 2.038262\end{array}$
$\begin{array}{llll}\text { C } & 2.447157 & 0.142166 & 1.258280\end{array}$
C $2.347345-0.157643 \quad-0.120776$
$\begin{array}{llll}\text { C } & 1.048397 & -0.308890 & -0.661379\end{array}$
$\begin{array}{llll}\text { C } & 3.477676 & -0.297557 & -0.947098\end{array}$ 


$\begin{array}{lccc}\text { C } & 4.853449 & -0.216829 & -0.483266 \\ \text { C } & 5.449151 & -1.275191 & 0.395069 \\ \text { N } & 6.838636 & -1.568076 & 0.061889 \\ \text { C } & 7.146662 & -1.350025 & -1.140979 \\ \text { C } & 6.173273 & -0.907978 & -2.175981 \\ \text { S } & 6.627530 & 0.520224 & -3.149144 \\ \text { O } & 7.864047 & 0.180686 & -3.805003 \\ \text { C } & 5.427335 & -1.816695 & -2.856436 \\ \text { O } & 5.496429 & 0.896396 & -3.951890 \\ \text { C } & 6.987110 & 1.832872 & -2.032838 \\ \text { H } & 4.869178 & -2.203308 & 0.329412 \\ \text { H } & 5.418547 & -0.971297 & 1.447414 \\ \text { H } & 8.171346 & -1.532044 & -1.472003 \\ \text { H } & 3.301977 & -0.494760 & -2.004768 \\ \text { H } & 5.278049 & 0.775442 & -0.326518 \\ \text { H } & 0.949731 & -0.534334 & -1.718403 \\ \text { H } & -1.054125 & -0.302254 & -0.309955 \\ \text { H } & -0.828800 & 0.210785 & 2.103679 \\ \text { H } & 1.421179 & 0.503299 & 3.092217 \\ \text { H } & 3.426909 & 0.286453 & 1.701626 \\ \text { H } & 4.887673 & -1.545822 & -3.755818 \\ \text { H } & 5.313027 & -2.825993 & -2.476812 \\ \text { H } & 7.476855 & 2.591577 & -2.643426 \\ \text { H } & 6.071478 & 2.233821 & -1.605567 \\ \text { H } & 7.673981 & 1.486673 & -1.261040\end{array}$

\section{4}

32

scf done: -1107.530657

C $\quad-0.293565 \quad 0.044279 \quad 0.611012$

$\begin{array}{llll}\text { C } & -0.101461 & 0.762616 & 1.784991\end{array}$

C $1.184453 \quad 1.204940 \quad 2.089598$

C $2.244603 \quad 0.932946 \quad 1.243492$

$\begin{array}{llll}\text { C } & 2.039465 & 0.213279 & 0.075813\end{array}$

$\begin{array}{llll}\text { C } & 0.766485 & -0.230122 & -0.238544\end{array}$

$\begin{array}{llll}\text { C } & -1.244184 & 1.028668 & 2.708355\end{array}$

C $\quad-1.118493 \quad 0.532205 \quad 4.176497$

$\begin{array}{llll}\text { C } & -1.638316 & 1.907911 & 4.640012\end{array}$

C $\quad-3.044293 \quad 1.561513 \quad 5.042803$

$\begin{array}{llll}\mathrm{N} & -3.338697 & 0.333002 & 5.043960\end{array}$

$\begin{array}{llll}\text { C } & -2.164162 & -0.455767 & 4.665786\end{array}$

$\begin{array}{llll}\text { C } & -1.459434 & 2.472812 & 3.224557\end{array}$

$\begin{array}{llll}\text { O } & 0.651553 & 2.846865 & 5.466392\end{array}$

S $\quad-0.692555 \quad 2.658054 \quad 5.946615$

$\begin{array}{llll}\text { O } & -0.892226 & 1.866965 & 7.133024\end{array}$

C $\quad-1.424888 \quad 4.242656 \quad 6.167912$

H $\quad-1.836151 \quad-1.022558 \quad 5.542241$

H $-2.442720 \quad-1.184294 \quad 3.899252$

$\begin{array}{llll}H & -3.779027 & 2.323545 & 5.301269\end{array}$

$\begin{array}{llll}H & -2.307887 & 3.023341 & 2.817525\end{array}$

H $\quad-0.559806 \quad 3.084563 \quad 3.141117$

$\begin{array}{llll}H & -0.094484 & 0.292823 & 4.464424\end{array}$

$\begin{array}{llll}H & -2.167908 & 0.638333 & 2.270080\end{array}$

$\begin{array}{llll}\text { H } & -1.290527 & -0.305851 & 0.362350\end{array}$

H $\quad 0.595327 \quad-0.792976 \quad-1.148997$

H $2.870677 \quad 0.000491 \quad-0.586219$

$\begin{array}{llll}H & 3.238496 & 1.284119 & 1.496463\end{array}$

H $1.360156 \quad 1.766672 \quad 3.003256$

$\begin{array}{llll}H & -0.818106 & 4.768924 & 6.904336\end{array}$

$\begin{array}{llll}H & -1.404692 & 4.774425 & 5.216944\end{array}$

$\begin{array}{llll}H & -2.443703 & 4.127748 & 6.534056\end{array}$

TS (V-VIII) 
scf done: -1695.985472

C $0.694764 \quad-0.037657 \quad-0.411990$

$\begin{array}{llll}\text { C } & 0.773102 & -0.862791 & 0.700085\end{array}$

$\begin{array}{llll}\text { C } & 1.899369 & -0.845529 & 1.501312\end{array}$

C $2.966352 \quad 0.002542 \quad 1.209896$

C $2.882425 \quad 0.813828 \quad 0.081015$

$\begin{array}{llll}\text { C } & 1.755158 & 0.798397 & -0.721367\end{array}$

$\begin{array}{llll}\text { C } & 4.175920 & 0.030596 & 2.041747\end{array}$

C $\quad 4.160240 \quad-0.233345 \quad 3.392121$

C $5.420809 \quad-0.350521 \quad 4.158259$

$\begin{array}{llll}\text { N } & 5.671187 & -1.784081 & 4.484485\end{array}$

C $5.351443 \quad-2.804072 \quad 3.607742$

$\begin{array}{llll}\text { C } & 5.202016 & -2.723723 & 2.266452\end{array}$

C $5.421934-1.600023 \quad 1.400245$

S $\quad 5.845925 \quad-2.185906 \quad 6.100363$

C $4.206285-2.122649 \quad 6.730382$

$\begin{array}{llll}\text { O } & 6.614906 & -1.143131 & 6.700495\end{array}$

$\begin{array}{llll}\text { O } & 6.304310 & -3.538018 & 6.146262\end{array}$

$\begin{array}{llll}\text { S } & 4.648918 & -4.210117 & 1.492674\end{array}$

$\begin{array}{lllll}\text { O } & 3.637074 & -3.869856 & 0.525872\end{array}$

$\begin{array}{llll}\text { C } & 6.066214 & -4.774187 & 0.608018\end{array}$

$\begin{array}{llll}\text { O } & 4.333640 & -5.192372 & 2.497749\end{array}$

$\begin{array}{llll}H & 5.395738 & 0.186787 & 5.107770\end{array}$

H $\quad 6.273190 \quad 0.043467 \quad 3.601289$

$\begin{array}{llll}H & 4.249818 & -2.408099 & 7.780927\end{array}$

$\begin{array}{llll}H & 3.823425 & -1.107432 & 6.635888\end{array}$

H $3.590566 \quad-2.826471 \quad 6.171073$

H $\quad 5.220116-3.7723334 .076987$

$\begin{array}{llll}H & 4.969709 & 0.674021 & 1.667388\end{array}$

H $3.263574 \quad-0.6258963 .865282$

$\begin{array}{llll}H & 3.714008 & 1.467717 & -0.162503\end{array}$

H $\quad 1.704788 \quad 1.440977 \quad-1.592693$

H $\quad-0.188010 \quad-0.053428 \quad-1.040324$

H $\quad-0.046806-1.5302060 .938936$

H $\quad 1.964222 \quad-1.517326 \quad 2.351223$

H $\quad 5.116572 \quad-1.698173 \quad 0.364175$

H $6.339681-1.0427601 .546346$

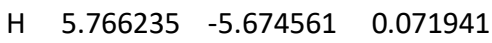

H $\quad 6.379715 \quad-4.003616 \quad-0.094685$

H $\quad 6.860343 \quad-4.997594 \quad 1.318331$

${ }^{2} \mathrm{VIII}$

39

scf done: -1696.024923

C $0.655965 \quad-0.570210 \quad 0.399152$

C $0.972042 \quad-0.966362 \quad 1.687521$

$\begin{array}{llll}\text { C } & 2.257557 & -0.790245 & 2.175066\end{array}$

$\begin{array}{llll}\text { C } & 3.245595 & -0.218778 & 1.383621\end{array}$

$\begin{array}{llll}\text { C } & 2.913943 & 0.180860 & 0.093688\end{array}$

$\begin{array}{llll}\text { C } & 1.632587 & 0.007863 & -0.397817\end{array}$

$\begin{array}{llll}\text { C } & 4.674437 & -0.086468 & 1.858441\end{array}$

$\begin{array}{llll}\text { C } & 4.782795 & 0.135910 & 3.323841\end{array}$

$\begin{array}{llll}C & 5.803313 & -0.534013 & 4.154673\end{array}$

$\begin{array}{llll}\mathrm{N} & 5.458087 & -1.957153 & 4.433815\end{array}$

$\begin{array}{llll}\text { C } & 5.145234 & -2.824547 & 3.415441\end{array}$

C $5.163246-2.570910 \quad 2.099362$

C $5.512686-1.289205 \quad 1.394385$

S $5.109596 \quad-2.397799 \quad 6.006132$

C $3.474978-1.800660 \quad 6.246509$

$\begin{array}{llll}\text { O } & 5.995451 & -1.659385 & 6.848160\end{array}$

$\begin{array}{llll}\text { O } & 5.097562 & -3.825356 & 6.051362\end{array}$

$\begin{array}{llll}\text { S } & 4.742361 & -3.935187 & 1.078446\end{array}$ 


$\begin{array}{lrrr}\text { O } & 3.886106 & -3.472803 & 0.015787 \\ \mathrm{C} & 6.278309 & -4.406844 & 0.351153 \\ \mathrm{O} & 4.283459 & -5.030079 & 1.894350 \\ \mathrm{H} & 5.920858 & -0.041346 & 5.117928 \\ \mathrm{H} & 6.791466 & -0.537132 & 3.685607 \\ \mathrm{H} & 3.203610 & -1.989113 & 7.284750 \\ \mathrm{H} & 3.458252 & -0.730289 & 6.041363 \\ \mathrm{H} & 2.805706 & -2.338660 & 5.576121 \\ \mathrm{H} & 4.864826 & -3.817328 & 3.749435 \\ \mathrm{H} & 5.095933 & 0.790696 & 1.340213 \\ \mathrm{H} & 4.120356 & 0.860457 & 3.785407 \\ \mathrm{H} & 3.678226 & 0.631587 & -0.533383 \\ \mathrm{H} & 1.393164 & 0.327528 & -1.405535 \\ \mathrm{H} & -0.349178 & -0.706381 & 0.017529 \\ \mathrm{H} & 0.214476 & -1.415981 & 2.319148 \\ \mathrm{H} & 2.498461 & -1.105014 & 3.186078 \\ \mathrm{H} & 5.352409 & -1.411561 & 0.321000 \\ \mathrm{H} & 6.577264 & -1.068385 & 1.514567 \\ \mathrm{H} & 6.061337 & -5.199027 & -0.365547 \\ \mathrm{H} & 6.712117 & -3.549373 & -0.161935 \\ \mathrm{H} & 6.944284 & -4.766836 & 1.133334\end{array}$

\section{TS (VIII-IX)}

39

scf done: -1695.982428

$\begin{array}{llll}\text { C } & -0.244712 & 0.081370 & -0.079057\end{array}$

C $\quad 0.010182 \quad 0.118206 \quad 1.290201$

C $1.318222 \quad-0.046472 \quad 1.727518$

$\begin{array}{llll}\text { C } & 2.351049 & -0.244206 & 0.824559\end{array}$

$\begin{array}{llll}\text { C } & 2.087342 & -0.276815 & -0.533859\end{array}$

$\begin{array}{llll}\text { C } & 0.785327 & -0.113132 & -0.982348\end{array}$

$\begin{array}{llll}\text { C } & -1.096380 & 0.350533 & 2.276278\end{array}$

$\begin{array}{llll}\text { C } & -2.290395 & -0.605469 & 2.194937\end{array}$

$\begin{array}{llll}\text { C } & -3.495859 & 0.334604 & 2.078427\end{array}$

C $\quad-1.842101 \quad 1.653545 \quad 2.094813$

$\begin{array}{llll}\text { C } & -2.211815 & 2.380113 & 3.329597\end{array}$

$\begin{array}{llll}\text { N } & -3.241672 & 1.551090 & 4.037079\end{array}$

$\begin{array}{llll}\text { C } & -4.118848 & 0.930456 & 3.158005\end{array}$

S $\quad-4.525673 \quad 0.092503 \quad 0.664320$

$\begin{array}{llll}\mathrm{O} & -5.605743 & 1.042091 & 0.702412\end{array}$

$\begin{array}{llll}\text { S } & -3.604153 & 1.802006 & 5.624899\end{array}$

$\begin{array}{llll}\text { C } & -2.746471 & 0.495441 & 6.425021\end{array}$

$\begin{array}{llll}\text { C } & -5.208062 & -1.518139 & 0.895533\end{array}$

$\begin{array}{lllll}\text { O } & -3.680403 & 0.060220 & -0.501829\end{array}$

$\begin{array}{llll}0 & -3.006841 & 3.044315 & 6.007129\end{array}$

$\begin{array}{llll}O & -5.010005 & 1.596196 & 5.793313\end{array}$

$\begin{array}{llll}H & -2.629359 & 3.368147 & 3.123126\end{array}$

H $\quad-1.351599 \quad 2.499545 \quad 3.995016$

$\begin{array}{llll}H & -5.173341 & 1.181068 & 3.193511\end{array}$

H $\quad-2.390696 \quad-1.267059 \quad 3.059576$

$\begin{array}{llll}\text { H } & -2.223041 & -1.232969 & 1.303634\end{array}$

$\begin{array}{llll}\mathrm{H} & -1.722087 & 2.219417 & 1.177231\end{array}$

$\begin{array}{llll}H & -0.667740 & 0.328888 & 3.285415\end{array}$

H $\quad 1.529245 \quad-0.019490 \quad 2.791984$

H $\quad 3.365087-0.371901 \quad 1.185716$

H $\quad 2.892526 \quad-0.429135 \quad-1.243111$

H $\quad 0.571062 \quad-0.136643 \quad-2.044811$

$\begin{array}{llll}\text { H } & -1.261990 & 0.205774 & -0.439930\end{array}$

H $\quad-5.823073 \quad-1.7264390 .019873$

$\begin{array}{llll}\mathrm{H} & -4.407515 & -2.252161 & 0.971128\end{array}$

$\begin{array}{llll}\mathrm{H} & -5.817907 & -1.512444 & 1.797854\end{array}$

$\begin{array}{llll}H & -2.931816 & 0.579741 & 7.495427\end{array}$

H $\quad-3.126350 \quad-0.451580 \quad 6.043545$ 
${ }^{2} \mathrm{IX}$

39

scf done: -1696.028895

C $\quad-2.033167 \quad-0.424624 \quad 4.939095$

C $-1.123625 \quad 0.632974 \quad 4.355379$

$\begin{array}{llll}\text { C } & -1.651800 & 2.006103 & 4.827667\end{array}$

C $-3.031196 \quad 1.707678 \quad 5.235001$

$\begin{array}{llll}\text { N } & -3.242454 & 0.334306 & 5.307907\end{array}$

C $\quad-1.360516 \quad 1.107280 \quad 2.896675$

$\begin{array}{llll}\text { C } & -1.444771 & 2.564471 & 3.407794\end{array}$

$\begin{array}{llll}\text { S } & -0.662725 & 2.732842 & 6.141075\end{array}$

C $\quad-1.450470 \quad 4.273558 \quad 6.462686$

$\begin{array}{llll}\text { C } & -0.341183 & 0.749826 & 1.867152\end{array}$

$\begin{array}{llll}\text { C } & -0.697895 & 0.011979 & 0.745117\end{array}$

C $\quad 0.245772 \quad-0.333441 \quad-0.209222$

$\begin{array}{llll}\text { C } & 1.564740 & 0.056186 & -0.052679\end{array}$

C $1.934296 \quad 0.790223 \quad 1.064417$

C $0.989825 \quad 1.132368 \quad 2.015445$

$\begin{array}{llll}\text { O } & 0.657319 & 2.993142 & 5.624151\end{array}$

$\begin{array}{llll}\text { O } & -0.785767 & 1.882498 & 7.297634\end{array}$

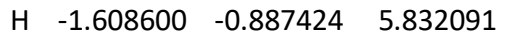

H $\quad-2.271537 \quad-1.216990 \quad 4.225423$

$\begin{array}{llll}\mathrm{H} & -3.844454 & 2.398057 & 5.399468\end{array}$

$\begin{array}{llll}H & -2.244627 & 3.190785 & 3.014607\end{array}$

H $\quad-0.489046 \quad 3.084293 \quad 3.316381$

$\begin{array}{llll}\mathrm{H} & -0.067103 & 0.458784 & 4.564457\end{array}$

$\begin{array}{llll}H & -2.348819 & 0.774285 & 2.563096\end{array}$

H $\quad-1.731958 \quad-0.293857 \quad 0.618622$

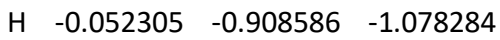

H $2.304724 \quad-0.210402 \quad-0.798094$

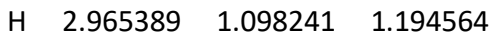

$\begin{array}{llll}H & 1.292265 & 1.706598 & 2.887050\end{array}$

$\begin{array}{llll}H & -0.846656 & 4.789989 & 7.208184\end{array}$

H $\quad-1.480352 \quad 4.849848 \quad 5.537968$

$\begin{array}{llll}H & -2.452728 & 4.091140 & 6.846956\end{array}$

S $-4.751460 \quad-0.323860 \quad 5.197589$

$\begin{array}{llll}0 & -4.617436 & -1.702628 & 5.548072\end{array}$

$\begin{array}{llll}\text { O } & -5.627668 & 0.533787 & 5.932129\end{array}$

$\begin{array}{llll}\text { C } & -5.169908 & -0.203710 & 3.492231\end{array}$

$\begin{array}{llll}\text { H } & -6.194929 & -0.554811 & 3.378672\end{array}$

$\begin{array}{llll}H & -5.094551 & 0.841986 & 3.192367\end{array}$

$\begin{array}{llll}H & -4.494754 & -0.829378 & 2.909788\end{array}$

\section{TS (IX-4)}

39

scf done: -1696.003826

$\begin{array}{llll}\text { C } & -0.099932 & -0.031188 & 0.100321\end{array}$

$\begin{array}{llll}\text { C } & -0.118658 & 0.067706 & 1.489541\end{array}$

C $\quad 1.091263 \quad 0.069249 \quad 2.172504$

C $\quad 2.293155 \quad-0.024827 \quad 1.488980$

$\begin{array}{llll}\text { C } & 2.299550 & -0.120539 & 0.108057\end{array}$

C $1.098142 \quad-0.123189-0.584960$

$\begin{array}{llll}\text { C } & -1.409590 & 0.178817 & 2.229977\end{array}$

C $-2.512724 \quad-0.872449 \quad 1.958020$

$\begin{array}{llll}\text { C } & -3.539257 & 0.272654 & 1.890936\end{array}$

$\begin{array}{llll}\text { C } & -2.394349 & 1.305927 & 1.820308\end{array}$

$\begin{array}{llll}\text { C } & -2.740548 & 2.351491 & 2.861039\end{array}$

$\begin{array}{llll}\mathrm{N} & -3.740944 & 1.716338 & 3.736700\end{array}$

$\begin{array}{llll}\text { C } & -4.201552 & 0.637578 & 3.173855\end{array}$

$\begin{array}{llll}\text { S } & -4.643946 & 0.297096 & 0.486708\end{array}$

$\begin{array}{llll}\text { O } & -5.358110 & 1.546122 & 0.520785\end{array}$ 


$\begin{array}{rrrr}\text { S } & -3.096801 & 1.567534 & 5.743988 \\ \text { C } & -1.607131 & 0.624383 & 6.084094 \\ \text { C } & -5.760308 & -1.026177 & 0.792664 \\ \text { O } & -3.871158 & -0.020045 & -0.685475 \\ \text { O } & -2.869605 & 2.939757 & 6.125475 \\ \text { O } & -4.204263 & 0.843366 & 6.317261 \\ \text { H } & -3.201143 & 3.239233 & 2.420639 \\ \text { H } & -1.879858 & 2.684666 & 3.446829 \\ \text { H } & -4.955670 & 0.032396 & 3.668231 \\ \text { H } & -2.682409 & -1.625267 & 2.728426 \\ \text { H } & -2.386972 & -1.362718 & 0.990601 \\ \text { H } & -2.193565 & 1.713469 & 0.829378 \\ \text { H } & -1.205951 & 0.244276 & 3.302940 \\ H & 1.088384 & 0.144381 & 3.255791 \\ \text { H } & 3.227445 & -0.023062 & 2.038544 \\ \text { H } & 3.238063 & -0.193685 & -0.428929 \\ \text { H } & 1.095633 & -0.197385 & -1.666240 \\ \text { H } & -1.035889 & -0.034835 & -0.452052 \\ H & -6.406198 & -1.101876 & -0.081708 \\ \text { H } & -5.189388 & -1.945960 & 0.918207 \\ \text { H } & -6.352282 & -0.803768 & 1.678868 \\ \text { H } & -1.510667 & 0.566844 & 7.169604 \\ \text { H } & -1.734276 & -0.370618 & 5.658867 \\ \text { H } & -0.756758 & 1.144219 & 5.646273\end{array}$

\section{TS (IV-X)}

32

scf done: -1107.443806

C $\quad-0.035018-0.314541 \quad-0.058359$

$\begin{array}{llll}\text { C } & -0.103259 & -0.151249 & 1.435417\end{array}$

$\begin{array}{llll}\text { C } & 1.390434 & 0.063234 & 1.778283\end{array}$

$\begin{array}{llll}\mathrm{N} & 2.089425 & 0.343099 & 0.530659\end{array}$

$\begin{array}{llll}\text { C } & 1.292096 & 0.113389 & -0.440222\end{array}$

$\begin{array}{llll}\text { C } & -0.766828 & -1.265963 & 2.146963\end{array}$

$\begin{array}{llll}\text { C } & -1.764157 & -1.167728 & 3.130470\end{array}$

$\begin{array}{llll}\text { C } & -2.294195 & 0.061058 & 3.593158\end{array}$

$\begin{array}{llll}\text { C } & -3.270456 & 0.091714 & 4.563547\end{array}$

$\begin{array}{llll}\text { C } & -3.762994 & -1.086387 & 5.115630\end{array}$

$\begin{array}{llll}\text { C } & -3.258366 & -2.308453 & 4.678867\end{array}$

$\begin{array}{llll}\text { C } & -2.282613 & -2.351839 & 3.711681\end{array}$

C $-1.035178-0.691391-0.882936$

S $-2.222110 \quad 1.580384 \quad-1.006604$

$\begin{array}{lllll}\text { O } & -1.169012 & 2.570088 & -1.128999\end{array}$

C $\quad-3.276164 \quad 1.716722 \quad-2.449072$

$\begin{array}{llll}\mathrm{O} & -3.072188 & 1.614933 & 0.170281\end{array}$

$\begin{array}{llll}H & -0.633303 & 0.793119 & 1.623601\end{array}$

$\begin{array}{llll}H & 1.605767 & 0.216608 & -1.477568\end{array}$

$\begin{array}{llll}\text { H } & 1.827491 & -0.838163 & 2.223657\end{array}$

$\begin{array}{llll}H & 1.549087 & 0.873402 & 2.492475\end{array}$

$\begin{array}{llll}\mathrm{H} & -0.419101 & -2.265112 & 1.889214\end{array}$

$\begin{array}{llll}\text { H } & -1.890341 & -3.306161 & 3.374777\end{array}$

$\begin{array}{llll}H & -3.635544 & -3.231886 & 5.103572\end{array}$

H $\quad-4.531183 \quad-1.052653 \quad 5.878760$

$\begin{array}{llll}\text { H } & -3.657828 & 1.046670 & 4.900374\end{array}$

$\begin{array}{llll}H & -1.928730 & 0.994643 & 3.181183\end{array}$

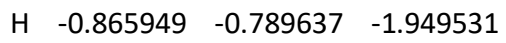

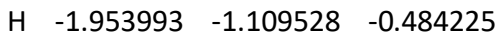

$\begin{array}{llll}H & -3.737300 & 2.704139 & -2.403709\end{array}$

H $\quad-2.652149 \quad 1.615235 \quad-3.333807$

H $\quad-4.025550 \quad 0.930760 \quad-2.395045$

${ }^{3} \mathrm{X}$

32 
scf done: -1107.475888

$\begin{array}{llll}\text { C } & 1.579722 & 0.371556 & 1.605514\end{array}$

$\begin{array}{llll}\text { C } & 0.379325 & -0.182802 & 1.098828\end{array}$

$\begin{array}{llll}\text { C } & 0.355480 & -0.515840 & -0.278592\end{array}$

$\begin{array}{llll}\text { C } & 1.451567 & -0.317098 & -1.083926\end{array}$

$\begin{array}{llll}\text { C } & 2.621307 & 0.226005 & -0.559268\end{array}$

$\begin{array}{llll}\text { C } & 2.669909 & 0.568234 & 0.788354\end{array}$

$\begin{array}{llll}\text { C } & -0.765099 & -0.413241 & 1.880251\end{array}$

$\begin{array}{llll}\text { C } & -0.927595 & -0.156119 & 3.332997\end{array}$

C $\quad-2.053266 \quad 0.845454 \quad 3.690504$

$\begin{array}{llll}\text { N } & -2.895274 & 0.241511 & 4.708735\end{array}$

$\begin{array}{llll}\text { C } & -2.484535 & -0.988037 & 4.877303\end{array}$

C $-1.381471 \quad-1.358477 \quad 4.106254$

C $\quad-0.728233 \quad-2.673754 \quad 4.072103$

H $\quad 0.0232590 .185556 \quad 3.760700$

H $\quad-2.988937 \quad-1.657730 \quad 5.570456$

$\begin{array}{llll}H & -2.673254 & 1.071989 & 2.814083\end{array}$

$\begin{array}{llll}H & -1.665010 & 1.803900 & 4.044515\end{array}$

H $\quad-1.639065 \quad-0.821345 \quad 1.373676$

H $\quad-0.554844 \quad-0.939473 \quad-0.691072$

H $\quad 1.403458-0.586108-2.132970$

H $3.484867 \quad 0.382517 \quad-1.194359$

$\begin{array}{llll}\text { H } & 3.576670 & 0.994687 & 1.202481\end{array}$

$\begin{array}{llll}H & 1.646462 & 0.646511 & 2.651727\end{array}$

$\begin{array}{llll}\text { H } & -1.384485 & -3.475007 & 4.419861\end{array}$

$\begin{array}{llll}\text { H } & -0.342104 & -2.915197 & 3.075915\end{array}$

S $0.697208 \quad-2.684320 \quad 5.172061$

C $\quad 1.336351 \quad-4.314054 \quad 4.993730$

$\begin{array}{llll}\text { O } & 1.663546 & -1.747321 & 4.662255\end{array}$

$\begin{array}{llll}\mathrm{O} & 0.220659 & -2.513305 & 6.518439\end{array}$

H $\quad 2.212603 \quad-4.375777 \quad 5.638601$

H $\quad 0.581775 \quad-5.031281 \quad 5.312035$

H $\quad 1.620213 \quad-4.474711 \quad 3.954922$

\section{TS (IV-XI)}

32

scf done: -1107.434856

$\begin{array}{llll}\text { C } & -0.155016 & -0.169951 & 0.404597\end{array}$

$\begin{array}{llll}\text { C } & -0.069776 & 0.265772 & 1.749640\end{array}$

$\begin{array}{llll}\text { C } & 1.175759 & 0.780547 & 2.184313\end{array}$

C $2.254206 \quad 0.839552 \quad 1.329964$

$\begin{array}{llll}\text { C } & 2.145732 & 0.395741 & 0.016326\end{array}$

$\begin{array}{llll}\text { C } & 0.929469 & -0.107472 & -0.437700\end{array}$

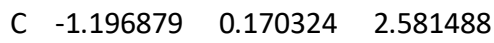

$\begin{array}{llll}\text { C } & -1.261097 & 0.573377 & 4.009065\end{array}$

$\begin{array}{llll}\text { C } & -1.891040 & 1.962308 & 4.156888\end{array}$

$\begin{array}{llll}\text { C } & -3.211252 & 1.703847 & 4.763443\end{array}$

$\begin{array}{llll}\mathrm{N} & -3.426193 & 0.507187 & 5.116280\end{array}$

C $\quad-2.242882 \quad-0.295232 \quad 4.824580$

$\begin{array}{llll}\text { C } & -1.640468 & 3.043387 & 3.338251\end{array}$

$\begin{array}{llll}\text { O } & 0.647545 & 2.570216 & 5.745796\end{array}$

S $-0.757903 \quad 2.602171 \quad 6.097116$

$\begin{array}{llll}\mathrm{O} & -1.202828 & 1.854015 & 7.256591\end{array}$

C $\quad-1.231085 \quad 4.302677 \quad 6.321430$

H $\quad-1.811062 \quad-0.626128 \quad 5.773035$

$\begin{array}{llll}H & -2.538463 & -1.196637 & 4.281686\end{array}$

$\begin{array}{llll}\mathrm{H} & -3.951819 & 2.492307 & 4.896211\end{array}$

$\begin{array}{llll}H & -2.304269 & 3.900735 & 3.341078\end{array}$

H $\quad-0.751553 \quad 3.092830 \quad 2.720219$

$\begin{array}{llll}H & -0.260339 & 0.555898 & 4.448602\end{array}$

H $\quad-2.124410 \quad-0.187875 \quad 2.138006$

H $\quad-1.101648 \quad-0.5634090 .048127$

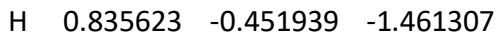




$\begin{array}{rrrr}H & 2.999654 & 0.444240 & -0.648610 \\ H & 3.196866 & 1.238285 & 1.687407 \\ H & 1.284368 & 1.141437 & 3.201690 \\ H & -0.731945 & 4.649379 & 7.226400 \\ H & -0.900364 & 4.867831 & 5.452440 \\ H & -2.313007 & 4.346703 & 6.437322\end{array}$

\section{${ }^{3} \mathrm{XI}$}

32

scf done: -1107.446096

$\begin{array}{llll}\text { C } & -0.219978 & -0.078377 & 0.488042\end{array}$

$\begin{array}{llll}\text { C } & -0.068616 & 0.296415 & 1.845083\end{array}$

$\begin{array}{llll}\text { C } & 1.193465 & 0.799351 & 2.242009\end{array}$

C $2.226412 \quad 0.908618 \quad 1.337503$

$\begin{array}{llll}\text { C } & 2.053216 & 0.527217 & 0.011145\end{array}$

$\begin{array}{llll}\text { C } & 0.819491 & 0.034060 & -0.404672\end{array}$

$\begin{array}{llll}\text { C } & -1.154771 & 0.163563 & 2.725633\end{array}$

C $\quad-1.156459 \quad 0.505658 \quad 4.167642$

C $\quad-1.682332 \quad 1.969061 \quad 4.418665$

C $\quad-3.075797 \quad 1.691918 \quad 4.915793$

$\begin{array}{llll}\mathrm{N} & -3.353960 & 0.493467 & 5.189608\end{array}$

$\begin{array}{llll}\text { C } & -2.182943 & -0.338033 & 4.947197\end{array}$

C $\quad-1.612497 \quad 2.925487 \quad 3.307639$

$\begin{array}{llll}\mathrm{O} & 0.662745 & 2.855483 & 5.289965\end{array}$

S $\quad-0.666756 \quad 2.647134 \quad 5.801815$

$\begin{array}{llll}\text { O } & -0.829490 & 1.806058 & 6.958589\end{array}$

C $\quad \begin{array}{llll}-1.391840 & 4.218650 & 6.134787\end{array}$

H $-1.794096 \quad-0.676181 \quad 5.911062$

H $-2.477216 \quad-1.232050 \quad 4.391728$

H $\quad-3.805803 \quad 2.494960 \quad 5.020683$

$\begin{array}{llll}H & -2.498519 & 3.461339 & 2.991035\end{array}$

$\begin{array}{llll}H & -0.670716 & 3.131380 & 2.816474\end{array}$

H $\quad-0.153130 \quad 0.393045 \quad 4.587364$

$\begin{array}{llll}H & -2.100206 & -0.178661 & 2.307660\end{array}$

H $-1.181100 \quad-0.462916 \quad 0.161906$

H $\quad 0.676350 \quad-0.262699 \quad-1.437439$

H $\quad 2.871171 \quad 0.616584 \quad-0.693684$

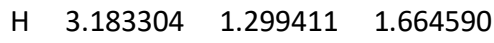

H $\quad 1.346468 \quad 1.117306 \quad 3.267520$

$\begin{array}{llll}H & -0.803391 & 4.660595 & 6.938555\end{array}$

$\begin{array}{llll}H & -1.336490 & 4.833814 & 5.237960\end{array}$

$\begin{array}{llll}\text { H } & -2.422587 & 4.080472 & 6.458515\end{array}$

$4^{\text {III }}$

32

scf done: -1107.532875

$\begin{array}{lrrr}\text { C } & -1.805971 & -0.410722 & 5.245281 \\ \text { C } & -0.836496 & 0.276897 & 4.312024 \\ \text { C } & -1.605243 & 1.436182 & 3.728389 \\ \text { C } & -2.957121 & 1.236668 & 4.304991 \\ \text { N } & -3.105376 & 0.245406 & 5.079465 \\ \text { C } & -1.176816 & 0.243077 & 2.865643 \\ \text { C } & -0.158432 & 0.394650 & 1.805397 \\ \text { C } & -0.502289 & 0.048279 & 0.500462 \\ \text { C } & 0.408231 & 0.166823 & -0.533760 \\ \text { C } & 1.685869 & 0.642252 & -0.284225 \\ \text { C } & 2.040702 & 0.994071 & 1.007138 \\ \text { C } & 1.129708 & 0.870618 & 2.043256 \\ \text { C } & -1.104182 & 2.820914 & 3.478858 \\ \text { S } & -0.376917 & 3.504067 & 4.958705 \\ \text { C } & -0.170043 & 5.206564 & 4.566458 \\ O & 0.926174 & 2.918212 & 5.142788 \\ O & -1.333888 & 3.384156 & 6.028078\end{array}$




$\begin{array}{crrr}H & -1.500987 & -0.318921 & 6.292462 \\ H & -1.904981 & -1.479955 & 5.035965 \\ H & -3.784008 & 1.905869 & 4.066033 \\ H & -1.925867 & 3.485581 & 3.197549 \\ H & -0.336864 & 2.867806 & 2.702231 \\ H & 0.200256 & 0.394129 & 4.598341 \\ H & -2.004614 & -0.404069 & 2.584604 \\ H & -1.501507 & -0.325600 & 0.301053 \\ H & 0.118941 & -0.114190 & -1.539849 \\ H & 2.401980 & 0.736445 & -1.091968 \\ H & 3.037824 & 1.365214 & 1.214121 \\ H & 1.433789 & 1.148186 & 3.047359 \\ H & 0.316477 & 5.666934 & 5.425935 \\ H & 0.463222 & 5.294173 & 3.684864 \\ H & -1.145634 & 5.658426 & 4.396336\end{array}$

\section{$4^{\mathrm{IV}}$}

32

scf done: -1107.529897

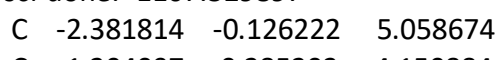

$\begin{array}{llll}\text { C } & -1.204097 & 0.285203 & 4.150884\end{array}$

$\begin{array}{llll}\text { C } & -1.611975 & 1.690984 & 3.750686\end{array}$

C $\quad-2.705711 \quad 2.023573 \quad 4.658462$

$\begin{array}{llll}\text { N } & -3.127360 & 1.080002 & 5.395785\end{array}$

$\begin{array}{llll}\text { C } & -0.896212 & -0.662470 & 2.994143\end{array}$

$\begin{array}{llll}\text { C } & 0.183870 & -0.178483 & 2.066678\end{array}$

$\begin{array}{llll}\text { C } & -0.058953 & -0.139123 & 0.700154\end{array}$

C $\quad 0.899077 \quad 0.345020 \quad-0.173493$

$\begin{array}{llll}\text { C } & 2.120295 & 0.782851 & 0.312029\end{array}$

$\begin{array}{llll}\text { C } & 2.378649 & 0.727110 & 1.672307\end{array}$

$\begin{array}{llll}\text { C } & 1.417529 & 0.248662 & 2.545842\end{array}$

$\begin{array}{llll}\text { C } & -1.147436 & 2.534842 & 2.835427\end{array}$

S $\quad-0.379662 \quad-2.322629 \quad 3.561102$

C $\quad-1.838979 \quad-3.179115 \quad 4.062714$

$\begin{array}{llll}\text { O } & 0.478881 & -2.167378 & 4.707292\end{array}$

$\begin{array}{llll}O & 0.126297 & -3.021466 & 2.409510\end{array}$

H $\quad-2.057362 \quad-0.632165 \quad 5.971188$

$\begin{array}{llll}H & -3.075270 & -0.802892 & 4.546030\end{array}$

H $\quad-3.140762 \quad 3.021419 \quad 4.697101$

H $\quad-1.593024 \quad 3.520182 \quad 2.749560$

$\begin{array}{llll}H & -0.337142 & 2.304765 & 2.156497\end{array}$

H $\quad-0.305233 \quad 0.362488 \quad 4.773166$

$\begin{array}{llll}\text { H } & -1.804810 & -0.851205 & 2.409805\end{array}$

$\begin{array}{llll}\text { H } & -1.017568 & -0.478416 & 0.320867\end{array}$

H $0.6898760 .381632 \quad-1.236033$

H $\quad 2.870770 \quad 1.166395 \quad-0.368963$

$\begin{array}{llll}H & 3.333466 & 1.064520 & 2.057871\end{array}$

H $\quad 1.628724 \quad 0.224023 \quad 3.608985$

H $\quad-1.518781 \quad-4.211593 \quad 4.205183$

H $\quad-2.224356 \quad-2.779100 \quad 4.996455$

H $\quad-2.582112 \quad-3.130060 \quad 3.268167$

${ }^{3} 4^{\mathrm{IV}}$

32

scf done: -1107.447703

C $\quad-2.472384 \quad-0.141554 \quad 5.022873$

$\begin{array}{llll}\text { C } & -1.236085 & 0.231573 & 4.184221\end{array}$

C $\quad-1.564884 \quad 1.659153 \quad 3.804166$

C $\quad-2.710671 \quad 2.010337 \quad 4.531563$

$\begin{array}{llll}\text { N } & -3.239939 & 1.066217 & 5.263319\end{array}$

$\begin{array}{llll}\text { C } & -0.933137 & -0.685831 & 2.996554\end{array}$

$\begin{array}{llll}\text { C } & 0.115416 & -0.164108 & 2.053131\end{array}$

$\begin{array}{llll}\text { C } & -0.158559 & -0.113275 & 0.692826\end{array}$ 


$\begin{array}{rrrr}\text { C } & 0.770144 & 0.400409 & -0.195420 \\ \text { C } & 1.993068 & 0.856309 & 0.268390 \\ \text { C } & 2.283228 & 0.789383 & 1.621731 \\ \text { C } & 1.351650 & 0.281817 & 2.510109 \\ \text { C } & -0.767888 & 2.537739 & 2.984123 \\ \text { S } & -0.376094 & -2.345130 & 3.516652 \\ \text { C } & -1.790790 & -3.193151 & 4.142026 \\ \text { O } & 0.572666 & -2.193819 & 4.589997 \\ \text { O } & 0.033596 & -3.042560 & 2.326648 \\ \text { H } & -2.217472 & -0.618564 & 5.973789 \\ \text { H } & -3.124599 & -0.841897 & 4.486059 \\ \text { H } & -3.144122 & 3.006810 & 4.514597 \\ \text { H } & -0.932496 & 2.632348 & 1.916038 \\ \text { H } & 0.047854 & 3.111896 & 3.412176 \\ \text { H } & -0.342936 & 0.249534 & 4.825127 \\ \text { H } & -1.851522 & -0.879757 & 2.429716 \\ \text { H } & -1.117330 & -0.469766 & 0.330152 \\ \text { H } & 0.537499 & 0.443841 & -1.252834 \\ \text { H } & 2.721220 & 1.260989 & -0.424579 \\ \text { H } & 3.240135 & 1.139882 & 1.990161 \\ \text { H } & 1.586832 & 0.247371 & 3.567808 \\ \text { H } & -1.458735 & -4.222158 & 4.282452 \\ \text { H } & -2.101911 & -2.771658 & 5.094038 \\ \text { H } & -2.592937 & -3.161029 & 3.406467\end{array}$

\section{TS (IV-XII)}

25

scf done: -518.907289

C $0.006197 \quad-0.217950 \quad-0.060600$

$\begin{array}{llll}\text { C } & -0.133847 & 0.216707 & 1.263844\end{array}$

C $1.034322 \quad 0.490701 \quad 1.990811$

C $2.281142 \quad 0.329348 \quad 1.424389$

$\begin{array}{llll}\text { C } & 2.401282 & -0.105672 & 0.110573\end{array}$

$\begin{array}{llll}\text { C } & 1.257876 & -0.376600 & -0.625349\end{array}$

$\begin{array}{llll}\text { C } & -1.418855 & 0.451222 & 1.877177\end{array}$

$\begin{array}{llll}\text { C } & -2.754475 & -0.029821 & 1.345025\end{array}$

$\begin{array}{llll}\text { C } & -3.642233 & -0.870344 & 2.268321\end{array}$

$\begin{array}{llll}\text { N } & -4.520523 & 0.058247 & 2.983736\end{array}$

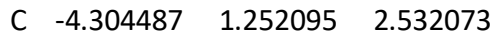

$\begin{array}{llll}\text { C } & -3.347775 & 1.340212 & 1.483564\end{array}$

$\begin{array}{llll}\text { C } & -2.321965 & 2.252781 & 1.246620\end{array}$

H $-2.684660 \quad-0.426715 \quad 0.331491$

$\begin{array}{llll}H & -4.818396 & 2.110774 & 2.959126\end{array}$

H $\quad-3.060390 \quad-1.449151 \quad 2.994560$

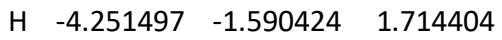

$\begin{array}{llll}\mathrm{H} & -1.412818 & 0.601404 & 2.955165\end{array}$

H $\quad 0.943376 \quad 0.831873 \quad 3.017240$

$\begin{array}{llll}H & 3.169096 & 0.541779 & 2.008719\end{array}$

H $3.380589-0.232932 \quad-0.335281$

H $\quad 1.342300 \quad-0.717071 \quad-1.651051$

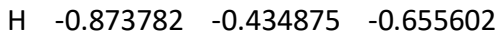

$\begin{array}{llll}H & -1.743167 & 2.177194 & 0.331227\end{array}$

H $\quad-2.305632 \quad 3.234743 \quad 1.714411$

\begin{tabular}{|c|c|c|c|}
\hline & & & \\
\hline & done: -518 & 939843 & \\
\hline C & -3.463347 & 1.161048 & 1.341872 \\
\hline C & -2.690528 & -0.109439 & 1.451504 \\
\hline C & -3.409026 & -0.857489 & 2.564748 \\
\hline $\mathrm{N}$ & -4.464119 & 0.033956 & 3.062419 \\
\hline c & -4.419912 & 1.142814 & 2.380785 \\
\hline & -1.463880 & 0.833387 & 1.726193 \\
\hline
\end{tabular}




$\begin{array}{lrrr}\text { C } & -0.130792 & 0.457005 & 1.168597 \\ \text { C } & 0.054718 & 0.257524 & -0.196854 \\ \text { C } & 1.291761 & -0.106086 & -0.698904 \\ \text { C } & 2.369250 & -0.278272 & 0.156757 \\ \text { C } & 2.197930 & -0.084328 & 1.516787 \\ \text { C } & 0.957528 & 0.280499 & 2.015299 \\ \text { C } & -2.262940 & 2.015894 & 1.089268 \\ \text { H } & -2.539606 & -0.677480 & 0.530119 \\ \text { H } & -5.073135 & 1.972817 & 2.639322 \\ \text { H } & -2.748136 & -1.118907 & 3.399845 \\ \text { H } & -3.857489 & -1.795515 & 2.224502 \\ \text { H } & -1.391982 & 0.995810 & 2.805865 \\ \text { H } & 0.827795 & 0.432536 & 3.082253 \\ \text { H } & 3.033243 & -0.215924 & 2.194981 \\ \text { H } & 3.337986 & -0.562568 & -0.237203 \\ \text { H } & 1.416272 & -0.255926 & -1.765282 \\ \text { H } & -0.778645 & 0.386687 & -0.880975 \\ \text { H } & -2.019052 & 2.124419 & 0.028996 \\ \text { H } & -2.209604 & 2.991124 & 1.572671\end{array}$


Comprehensive Table in A.U.

\begin{tabular}{|c|c|c|c|}
\hline Lacvp(d) values & H (Hartrees) & $\mathrm{S}\left(\mathrm{cal} / \mathrm{K}^{*} \mathrm{~mol}\right)$ & $\begin{array}{l}\text { Imaginary freq } \\
(1 / \mathrm{cm})\end{array}$ \\
\hline 3 & -1106.880894 & 135.836 & \\
\hline${ }^{3} 3$ & -1106.804725 & 136.17 & \\
\hline TS (3-III) & -1106.798218 & 133.323 & -492.4888 \\
\hline${ }^{3} \mid I I$ & -1106.875924 & 134.063 & \\
\hline TS (III-IV) & -1106.856009 & 135.658 & -286.159 \\
\hline${ }^{3} \mathrm{IV}$ & -1106.868117 & 144.636 & \\
\hline $3+{ }^{2} \mathrm{Ms}$ & -1695.181824 & 209.7 & \\
\hline TS (3-V) & -1695.190841 & 157.936 & -206.7432 \\
\hline${ }^{2} \mathrm{~V}$ & -1695.220438 & 162.604 & \\
\hline TS (V-VI) & -1695.191993 & 157.227 & -370.6295 \\
\hline VI & -1106.900501 & 135.884 & \\
\hline${ }^{3} \mathrm{VI}$ & -1106.823273 & 139.579 & \\
\hline TS (VI-7-endo) & -1106.805698 & 131.639 & -339.0999 \\
\hline TS (VI-VII) & -1106.81301 & 133.343 & -391.8719 \\
\hline${ }^{3} \mathrm{VII}$ & -1106.861394 & 127.706 & \\
\hline 4 & -1106.94178 & 125.232 & \\
\hline TS (V-VIII) & -1695.200220 & 152.807 & -647.6618 \\
\hline${ }^{2} \mathrm{VIII}$ & -1695.238531 & 152.176 & \\
\hline TS (VIII-IX) & -1695.200259 & 151.173 & -625.3631 \\
\hline${ }^{2} \mathrm{IX}$ & -1695.245717 & 151.476 & \\
\hline TS (IX-4) & -1695.232621 & 149.66 & -320.918 \\
\hline $4+{ }^{2} \mathrm{Ms}$ & -1695.242710 & 199.096 & \\
\hline TS (IV-XI) & -1106.854991 & 132.675 & -276.3483 \\
\hline${ }^{3} X I$ & -1106.861394 & 127.706 & \\
\hline TS (IV-X) & -1106.863467 & 136.151 & -163.3347 \\
\hline${ }^{3} x$ & -1106.888645 & 135.263 & \\
\hline $4^{\prime \prime \prime}$ & -1106.94181 & 122.855 & \\
\hline $4^{\mathrm{IV}}$ & -1106.938154 & 125.583 & \\
\hline $34^{I V}$ & -1106.862152 & 130.067 & \\
\hline
\end{tabular}




\begin{tabular}{|c|c|c|c|}
\hline Def2-tzvp values & H (Hartrees) & $\mathrm{S}\left(\mathrm{cal} / \mathrm{K}^{*} \mathrm{~mol}\right)$ & $\begin{array}{l}\text { Imaginary fre } \\
(1 / \mathrm{cm})\end{array}$ \\
\hline 1 & -1146.493303 & 144.27 & \\
\hline${ }^{3} 1$ & -1146.417069 & 139.018 & \\
\hline TS (1-II) & -1146.411644 & 132.997 & -285.928 \\
\hline${ }^{3} I 1$ & -1146.440102 & 138.747 & \\
\hline 2 & -1146.529487 & 133.083 & \\
\hline TS (1-II') & -1146.399568 & 135.39 & \\
\hline${ }^{3} \| I^{\prime}$ & -1146.479156 & 138.084 & -616.6099 \\
\hline $2^{\prime}$ & -1146.529369 & 132.194 & \\
\hline${ }^{2} \mathrm{Ms}$ & -588.30093 & 73.864 & \\
\hline 3 & -1107.228361 & 135.164 & \\
\hline${ }^{3} 3$ & -1107.151390 & 135.844 & \\
\hline TS (3-III) & -1107.14244 & 131.839 & -544.8373 \\
\hline${ }^{3} I I I$ & -1107.217777 & 133.285 & \\
\hline TS (III-IV) & -1107.185694 & 134.697 & -280.4895 \\
\hline${ }^{3} \mathrm{IV}$ & -1107.195668 & 144.53 & \\
\hline $3+{ }^{2} \mathrm{Ms}$ & -2293.008525 & 270.278 & \\
\hline TS (3-V) & -1695.668898 & 158.19 & -277.8522 \\
\hline${ }^{2} \mathrm{~V}$ & -1695.704138 & 164.403 & \\
\hline TS (V-VI) & -1695.672168 & 159.877 & -324.0021 \\
\hline $\mathrm{VI}$ & -1107.239316 & 136.464 & \\
\hline${ }^{3} \mathrm{VI}$ & -1107.160336 & 136.864 & \\
\hline TS (VI-VII) & -1107.148978 & 132.446 & -437.3805 \\
\hline${ }^{3} \mathrm{VII}$ & -1107.193643 & 130.303 & \\
\hline 4 & -1107.271870 & 122.944 & \\
\hline TS (V-VIII) & -1695.681673 & 152.53 & -679.0901 \\
\hline${ }^{2} \mathrm{VIII}$ & -1695.717872 & 150.157 & \\
\hline TS (VIII-IX) & -1695.677585 & 149.915 & -649.7728 \\
\hline${ }^{2} \mathrm{IX}$ & -1695.721683 & 149.626 & \\
\hline TS (IX-4) & -1695.698783 & 150.138 & -322.5286 \\
\hline $4+{ }^{2} \mathrm{Ms}$ & -1625.557631 & 234.246 & \\
\hline TS (IV-XI) & -1107.18351 & 131.509 & -320.8584 \\
\hline${ }^{3} \times 1$ & -1107.193643 & 130.303 & \\
\hline 4 & -1107.271870 & 122.944 & \\
\hline TS (IV-X) & -1107.192465 & 137.375 & -237.0548 \\
\hline${ }^{3} x$ & -1107.222111 & 134.163 & \\
\hline $4^{\prime \prime \prime}$ & -1107.274438 & 123.202 & \\
\hline $4^{\mathrm{IV}}$ & -1107.271834 & 124.862 & \\
\hline${ }^{3} 4^{\mathrm{IV}}$ & -1107.194543 & 130.161 & \\
\hline TS (IV-XII) & -518.70264 & 101.571 & -812.992 \\
\hline${ }^{2} \mathrm{XII}$ & -518.73281 & 102.039 & \\
\hline
\end{tabular}




\section{References}

[1] Gaussian 09, Revision A.1, Frisch, M. J.; Trucks, G. W.; Schlegel, H. B.; Scuseria, G. E.; Robb, M. A.; Cheeseman, J. R.; Scalmani, G.; Barone, V.; Mennucci, B.; Petersson, G. A.; Nakatsuji, H.; Caricato, M.; Li, X.; Hratchian, H. P.; Izmaylov, A. F.; Bloino, J.; Zheng, G.; Sonnenberg, J. L.; Hada, M.; Ehara, M.; Toyota, K.; Fukuda, R.; Hasegawa, J.; Ishida, M.; Nakajima, T.; Honda, Y.; Kitao, O.; Nakai, H.; Vreven, T.; Montgomery, Jr., J. A.; Peralta, J. E.; Ogliaro, F.; Bearpark, M.; Heyd, J. J.; Brothers, E.; Kudin, K. N.; Staroverov, V. N.; Kobayashi, R.; Normand, J.; Raghavachari, K.; Rendell, A.; Burant, J. C.; Iyengar, S. S.; Tomasi, J.; Cossi, M.; Rega, N.; Millam, N. J.; Klene, M.; Knox, J. E.; Cross, J. B.; Bakken, V.; Adamo, C.; Jaramillo, J.; Gomperts, R.; Stratmann, R. E.; Yazyev, O.; Austin, A. J.; Cammi, R.; Pomelli, C.; Ochterski, J. W.; Martin, R. L.; Morokuma, K.; Zakrzewski, V. G.; Voth, G. A.; Salvador, P.; Dannenberg, J. J.; Dapprich, S.; Daniels, A. D.; Farkas, Ö.; Foresman, J. B.; Ortiz, J. V.; Cioslowski, J.; Fox, D. J. Gaussian, Inc., Wallingford CT, 2009.

[2] Zhao, Y.; Truhlar, D. G. Theor. Chem. Account 2008, 120, 215.

[3] Hay, J. P.; Wadt, W. R. J. Chem. Phys. 1985, 82, 299. Friesner, R. A.; Murphy, R. B.; Beachy, M. D.; Ringlanda, M. N.; Pollard, W. T.; Dunietz, B. D.; Cao, Y. X. J. Phys. Chem. A 1999, 103, 1913; Weigen, F.; Ahlrichs, R. Phys. Chem. Chem. Phys. 2005, 7, 3297; Andrae, D.; Haeussermann, U.; Dolg, M.; Stoll, H.; Preuss, H. Theor. Chim. Acta 1990, 77, 123; Peterson, K. A.; Figgen, D.; Goll, E.; Stoll, H.; Dolg, M. J. Chem. Phys. 2003, 119, 11113.

[4] Barone. V.; Cossi, M. J. Phys. Chem. A 1998, 102, 1995; Cossi, M.; Rega, N.; Scalmani, G.; Barone, V. J. Comput. Chem. 2003, 24, 669; Klamt, A.; Schüürmann, G. J. Chem. Soc., Perkin Trans 2 1993, 799; Schäfer, A.; Klamt, A.; Sattel, D.; Lohrenz, J. C. W.; Eckert, F. Phys. Chem. Chem. Phys. 2000, 2, 2187.

[5] Kuang, J.; Ma, S. J. Am. Chem. Soc. 2010, 132, 1786.

[6] Lu, Z.; Yoon, T. P. Angew. Chem. Int. Ed. 2012, 51, 10329.

[7] Kuang, J.; Tang, X.; Ma, S. Org. Chem. Front. 2015, 2, 470.

[8] Krause, L.; Herbst-Irmer, R.; Sheldrick G.M.; Stalke D. J. Appl. Cryst. 2015, 48, 3. 
[9] Sheldrick, G. M. Acta Crystallogr. Sect. A Found. Crystallogr. 2015, A71, 3.

[10] Sheldrick, G. M. Acta Crystallogr. Sect. C Struct. Chem. 2015, C71, 3.

[11] Dolomanov, O. V.; Bourhis, L. J.; Gildea, R. J.; Howard, J. A. K.; Puschmann, H.; J. Appl. Cryst. 2009, 42, 339. 